\title{
Review Article \\ Second Order Equations in Functional Spaces: Qualitative and Discrete Well-Posedness
}

\author{
A. Ashyralyev, ${ }^{1}$ J. Pastor, $^{2}$ S. Piskarev, ${ }^{3}$ and H. A. Yurtsever ${ }^{4}$ \\ ${ }^{1}$ Department of Mathematics, Fatih University, Buyukcekmece, 34500 Istanbul, Turkey \\ ${ }^{2}$ Department of Applied Mathematics, University of Valencia, Burjassot, 46100 Valencia, Spain \\ ${ }^{3}$ Scientific Research Computer Center, Lomonosov Moscow State University, Vorobyovy Gory, Moscow 119899, Russia \\ ${ }^{4}$ American Islamic College, Chicago, IL 60613, USA \\ Correspondence should be addressed to J. Pastor; pastorv@uv.es
}

Received 29 September 2014; Accepted 31 December 2014

Academic Editor: Valery Covachev

Copyright (c) 2015 A. Ashyralyev et al. This is an open access article distributed under the Creative Commons Attribution License, which permits unrestricted use, distribution, and reproduction in any medium, provided the original work is properly cited.

\begin{abstract}
The present survey contains the recent results on the local and nonlocal well-posed problems for second order differential and difference equations. Results on the stability of differential problems for second order equations and of difference schemes for approximate solution of the second order problems are presented.
\end{abstract}

\section{Introduction}

The importance of coercive (maximal regularity, wellposedness) inequalities is well-known [1-6]. There are an extensive number of literatures which concern investigation of maximal regularity property for second order differential equations in time [7-11]. Recently [12], the well-posedness of difference schemes for abstract elliptic equations in $L^{p}([0, T] ; E)$ spaces was considered. The present survey is devoted to qualitative theory and the numerical analysis of abstract second order differential equations in functional spaces.

Let $B(E)$ denote the Banach algebra of all linear bounded operators on complex Banach space $E$. The set of all linear closed densely defined operators in $E$ will be denoted by $\mathscr{C}(E)$. We denote by $\sigma(B)$ the spectrum of the operator $B$ and by $\rho(B)$ the resolvent set of $B$.

Consider the following inhomogeneous Cauchy problem:

$$
\begin{gathered}
u^{\prime}(t)=A u(t)+f(t), \quad t \in[0, T] ; \\
u(0)=u^{0},
\end{gathered}
$$

in a Banach space $E$, where the operator $A \in \mathscr{C}(E)$ is the generator of a $C_{0}$-semigroup and $f(\cdot)$ is some function from
$[0, T]$ into $E$. Formally, as in the finite-dimensional analysis, problem (1) has a solution of the form

$$
\begin{array}{r}
u(t)=\exp (t A) u^{0}+\int_{0}^{t} \exp ((t-s) A) f(s) d s, \\
t \in[0, T] .
\end{array}
$$

This is the so-called constant variation formula. The properties of the expression $\int_{0}^{t} \exp ((t-s) A) f(s) d s$ and the corresponding interpretations of solutions related to representation (2) are of interest. Problem (1) can be considered in various functional spaces. The most popular situations are the following settings: the well-posedness in $C([0, T] ; E)$, $C^{\alpha, 0}([0, T] ; E)$, and $L^{p}([0, T] ; E)$ spaces (see $\left.[1,13]\right)$.

We say that problem (1) is well-posed, say in $C([0, T] ; E)$, if, for any $f(\cdot) \in C([0, T] ; E)$ and any $u_{0} \in D(A)$,

(i) the problem (1) is uniquely solvable; that is, $u(\cdot)$ satisfies the main equation and initial condition in (1), $u(\cdot)$ is continuously differentiable on $[0, T], u(t) \epsilon$ $D(A)$ for any $t \in[0, T]$, and $A u(\cdot)$ is continuous on $[0, T]$;

(ii) the operator $\left(f(\cdot), u^{0}\right) \rightarrow u(\cdot)$ as an operator from $C([0, T] ; E) \times D(A)$ to $C([0, T] ; E)$ is continuous. 
In the case $u^{0} \equiv 0$, the coercive well-posedness, for instance, in $C([0, T] ; E)$ means that

$$
\|A u(\cdot)\|_{C([0, T] ; E)} \leq c\|f(\cdot)\|_{C([0, T] ; E)} .
$$

In general, the coercive well-posedness in the space $\Upsilon([0, T] ; E)$ for problem (1) means that it is well-posed in the space $\Upsilon([0, T] ; E)$ and

$$
\begin{aligned}
& \left\|u^{\prime}(\cdot)\right\|_{\Upsilon([0, T] ; E)}+\|A u(\cdot)\|_{\Upsilon([0, T] ; E)} \\
& \quad \leq C\left(\|f(\cdot)\|_{\Upsilon([0, T] ; E)}+\left\|u^{0}\right\|_{\tilde{E}}\right),
\end{aligned}
$$

where $\widetilde{E}$ is some subspace of $E$. For results of the coercive well-posedness, see $[1,14]$.

We have to note that if (1) is coercively well-posed in the space $C([0, T] ; E)$, then the operator $A$ should be bounded or the space $E$ contains a subspace isomorphic to $c_{0}$ [15]. It means that in general problem (1) is not coercively well-posed in $C([0, T] ; E)$ space. However, problem (1) is classically wellposed in $C\left([0, T] ; E^{\alpha}\right)$ (see [16-18]), where $E^{\alpha}=(E, D(A))_{\alpha}$ is a suitable interpolation space. It is proved in [2] that coercive well-posedness in $C^{\alpha, 0}([0, T] ; E)$ space is equivalent to the condition that $A$ generates an analytic $C_{0}$-semigroup.

In the meantime, the situation in $L^{p}([0, T] ; E)$ space is not complete. One got only an extrapolation theorem and one could get a coercive inequality just for an interpolation space instead of $E$ (see [12]).

The necessary and sufficient conditions for coercive wellposedness of problem $(1)$ in $L^{p}([0, T] ; E)$ with $E$ to be a UMD space were obtained in [19-21].

Theorem 1 (see [21]). Let A generate a bounded analytic semigroup $\exp (\cdot A)$ on $U M D$ space $E$. Then problem (1) is coercively well-posed in the space $L^{p}\left(\mathbb{R}_{+} ; E\right)$ if and only if one of the sets, (i), (ii), or (iii), is R-bounded:

(i) $\left\{\lambda(\lambda I-A)^{-1}: \lambda \in i \mathbb{R}, \lambda \neq 0\right\}$;

(ii) $\{\exp (t A), t A \exp (t A): t>0\}$;

(iii) $\{\exp (z A):|\arg z| \leq \delta\}$.

When $E=C^{\theta}(\Omega)$, there is another "maximal regularity" result known. Let us consider the following mixed CauchyDirichlet parabolic problem:

$$
\begin{gathered}
\frac{\partial u}{\partial t}(t, x)=\mathscr{A} u(t, x)+f(t, x), \quad t \in[0, T], x \in \bar{\Omega}, \\
u\left(t, x^{\prime}\right)=g\left(t, x^{\prime}\right), \quad t \in[0, T], x^{\prime} \in \partial \Omega, \\
u(0, x)=u_{0}(x), \quad x \in \bar{\Omega} .
\end{gathered}
$$

Definition 2. One says that problem (5) has a strict solution if there is a continuous function $u(t, x)$ such that it has the first derivative in $t$ and the derivatives of order less than or equal to 2 in the space variables that are continuous up to the boundary of $[0, T] \times \bar{\Omega}$; that is, $u(\cdot) \in C^{1}([0, T] ; C(\bar{\Omega})) \cap$ $C\left([0, T] ; C^{2}(\bar{\Omega})\right)$, and the equations in (5) are satisfied.
By $B\left([0, T] ; C^{2 \theta}(\bar{\Omega})\right)$ we denote the space of bounded functions $u(\cdot):[0, T] \rightarrow C^{2 \theta}(\bar{\Omega})$ endowed with the usual sup-norm.

Theorem 3 (see [14]). Suppose the following assumptions are satisfied for some $\theta \in(0,2) \backslash\{1\}$ :

(I) $\Omega$ is an open bounded subset of $\mathbb{R}^{n}$ lying to one side of its topological boundary $\partial \Omega$, which is a submanifold of $\mathbb{R}^{n}$ of dimension $n-1$ and class $C^{2+2 \theta}$.

(II) The operator $\mathscr{A}=\mathscr{A}\left(x, \partial_{x}\right)=\sum_{|\alpha| \leq 2} a_{\alpha}(x) \partial_{x}^{\alpha}$ is a second order strongly elliptic operator (i.e., $\operatorname{Re}\left(\sum_{|\alpha|=2} a_{\alpha}(x) \xi^{\alpha}\right) \geq \nu|\xi|^{2}$ for some $\nu>0$ and for any $\left.(x, \xi) \in \bar{\Omega} \times \mathbb{R}^{n}\right)$ with coefficients of class $C^{2 \theta}(\bar{\Omega})$.

Then problem (5) has a unique strict solution $u(\cdot)$ belonging to $B\left([0, T] ; C^{2+2 \theta}(\bar{\Omega})\right)$ such that $\partial u / \partial t \in B\left([0, T] ; C^{2 \theta}(\bar{\Omega})\right)$ if and only if the following conditions are satisfied:

(a) $u_{0} \in C^{2+2 \theta}(\bar{\Omega})$;

(b) $f \in C([0, T] ; C(\bar{\Omega})) \cap B\left([0, T] ; C^{2 \theta}(\bar{\Omega})\right)$;

(c) $g \in B\left([0, T] ; C^{2+2 \theta}(\partial \Omega)\right) \cap C\left([0, T] ; C^{2}(\partial \Omega)\right) \cap C^{1}([0$, $T] ; C(\partial \Omega)), \partial g / \partial t \in B\left([0, T] ; C^{2 \theta}(\partial \Omega)\right), \partial g / \partial t-\gamma f \in$ $C^{\theta}([0, T] ; C(\partial \Omega))$;

(d) $\gamma u_{0}=g(0, \cdot)$;

(e) $(\partial g / \partial t)(0, \cdot)-\gamma f(0, \cdot)=\gamma \mathscr{A} u_{0}$.

Note that in $[4,5]$ the maximal regularity was proved for the problem

$$
\begin{array}{r}
\frac{d}{d t}\left(b_{0} u(t)+\int_{-\infty}^{t} \beta(t-s) u(s) d s\right)+a_{\infty} u(t) \\
=c_{0} A u(t)-\int_{-\infty}^{t} \gamma(t-s) A u(s) d s+f(t)
\end{array}
$$

in Hölder, Lebesgue, and Besov spaces.

\section{Hyperbolic Problems}

The situation in case of second order equation is very different from the first order equation. Let us consider the Cauchy problem

$$
\begin{gathered}
u^{\prime \prime}(t)=A u(t)+f(t), \quad t \in[0, T] ; \\
u(0)=u^{0}, \quad u^{\prime}(0)=u^{1},
\end{gathered}
$$

in a Banach space $E$, where the operator $A$ is the generator of a $C_{0}$-cosine operator function $C(\cdot, A)$. We will write $A \in$ $\mathscr{C}(M, \omega)$ if $\|C(t, A)\| \leq M e^{\omega|t|}, t \in \mathbb{R}$.

Definition 4. Function $u(\cdot)$ is called a classical solution of problem (7) if $u(\cdot)$ is twice continuously differentiable, $u(t) \epsilon$ $D(A)$ for all $t \in[0, T]$, and $u(\cdot)$ satisfies relations (7). 
Assume that $f(\cdot) \in C([0, T] ; E)$ and $u(\cdot)$ is a classical solution of (7). Considering the expression

$$
\frac{d}{d s}\left(C(t-s, A) u(s)+S(t-s, A) u^{\prime}(s)\right)=S(t-s, A) f(s)
$$

and integrating it in $0 \leq s<t$, we get

$$
\begin{aligned}
u(t)= & C(t, A) u^{0}+S(t, A) u^{1} \\
& +\int_{0}^{t} S(t-s, A) f(s) d s, \quad t \in[0, T],
\end{aligned}
$$

which is analogous to (2). As in the case of $C_{0}$-semigroups of operators, the function $u(\cdot)$ given by (9) is not a classical solution in general, since it can be not twice continuously differentiable.

Proposition 5 (see [22]). Let $A \in \mathscr{C}(M, \omega)$, and let either

$$
\begin{aligned}
& \text { (i) } f(\cdot), A f(\cdot) \in C([0, T) ; E) \text { and } f(t) \in D(A) \text { for } t \in \\
& {[0, T]} \\
& \text { or } \\
& \text { (ii) } f(\cdot) \in C^{1}([0, T] ; E) \text {. }
\end{aligned}
$$

Then the function $u(\cdot)$ from (9) with $u^{0} \in D(A)$ and $u^{1} \in$ $E^{1}$ is a classical solution of problem (7) on $[0, T]$. Here $E^{1}$ is the Kisynskii space; that is, $E^{1}$ is the space with the norm $\|x\|_{E^{1}}:=$ $\|x\|+\sup _{0<t \leq 1}\left\|C^{\prime}(t, A) x\right\|$.

Definition 6. The function $u(\cdot) \in C([0, T) ; E)$ given by expression (9) is called a mild solution of problem (7).

Let us consider the following homogenous uniformly well-posed Cauchy problem:

$$
\begin{gathered}
u^{\prime \prime}(t)=A u(t), \quad t \in \mathbb{R} ; \\
u(0)=u^{0}, \quad u^{\prime}(0)=u^{1} .
\end{gathered}
$$

Define the matrix operator $\mathscr{A}:=\left(\begin{array}{ll}0 & I \\ A & 0\end{array}\right): E^{1} \times E \rightarrow E^{1} \times E$ acting on an element $(x, y) \in E^{1} \times E$ by the formula $\mathscr{A}(x, y)=$ $(y, A x)$ that is given on the domain of $D(\mathscr{A})=D(A) \times E^{1}$. In what follows, an element $(x, y) \in E^{1} \times E$ will be written as the vector $\left(\begin{array}{l}x \\ y\end{array}\right)$.

The operator $\mathscr{A}$ generates the following $C_{0}$-groups of operators on the Banach space $E^{1} \times E[23]$ :

$$
\begin{aligned}
\exp (t \mathscr{A})\left(\begin{array}{l}
x \\
y
\end{array}\right) & :=\left(\begin{array}{cc}
C(t, A) & S(t, A) \\
A S(t, A) & C(t, A)
\end{array}\right)\left(\begin{array}{l}
x \\
y
\end{array}\right) \\
& =\left(\begin{array}{c}
C(t, A) x+S(t, A) y \\
A S(t, A) x+C(t, A) y
\end{array}\right), \quad t \in \mathbb{R} .
\end{aligned}
$$

We have to note that the study of problem (10) by reducing it to (equivalent) first order system some time is inconvenient, since the space $E^{1}$ is defined either through the $C_{0}$-cosine operator-valued function $C(\cdot, A)$ or through infinitely many powers of the resolvent. Therefore, certain additional conditions that allows us to reduce problem (10) to a first order system without use of the space $E^{1}$ are of interest [24].

Let uniformly well-posed problem (10) have the form

$$
\begin{gathered}
u^{\prime \prime}(t)=\mathfrak{B}^{2} u(t) \quad t \in \mathbb{R} ; \\
u(0)=u^{0}, \quad u^{\prime}(0)=u^{1},
\end{gathered}
$$

where $\mathfrak{B} \in \mathscr{C}(E)$.

Definition 7. One says that solution $u(\cdot)$ of problem (12) satisfies condition $(K)$ if $u^{\prime}(\cdot) \in C([0, T] ; \mathscr{D}(\mathfrak{B}))$.

Proposition 8 (see [25]). Problem (12) has a unique solution satisfying condition $(K)$ if and only if the following Cauchy problem is uniformly well-posed on the space $E \times E$ :

$$
\begin{aligned}
\left(\begin{array}{l}
u \\
v
\end{array}\right)^{\prime}(t)= & \left(\begin{array}{ll}
0 & \mathfrak{B} \\
\mathfrak{B} & 0
\end{array}\right)\left(\begin{array}{l}
u \\
v
\end{array}\right)(t), \quad t \in \mathbb{R}, \\
& \left(\begin{array}{l}
u \\
v
\end{array}\right)(0)=\left(\begin{array}{l}
u_{0} \\
v_{0}
\end{array}\right) .
\end{aligned}
$$

The following condition $(F)$, similar to previous condition $(K)$, will allows us to simplify the study of problem (10) by using $C_{0}$-semigroups.

Definition 9. $C_{0}$-cosine operator-valued function $C(\cdot, A)$ satisfies condition $(F)$ if the following conditions hold:

(i) There exists $\mathfrak{B} \in \mathscr{C}(E)$ such that $\mathfrak{B}^{2}=A$, and $\mathfrak{B}$ commutes with any operator from $B(E)$ commuting with $A$.

(ii) An operator $S(t, A)$ maps $E$ into $D(\mathfrak{B})$ for any $t \in \mathbb{R}$.

(iii) The function $\mathfrak{B S}(t, A) x$ is continuous in $t \in \mathbb{R}$ for any fixed $x \in E$.

Proposition 10 (see [22]). Under condition (F), for each $t \in$ $\mathbb{R}$, one has $\mathfrak{B S}(t, A) \in B(E)$ and $D(\mathfrak{B}) \subseteq E^{1}$.

Proposition 11 (see [22]). There exists Banach space E and $C_{0}$-cosine operator-valued function $C(\cdot, A)$ (even uniformly bounded) such that condition $(F)$ does not hold.

Proposition 12 (see [26]). Let the space E be Hilbert, and let the operator $A$ be self-adjoint and negative-definite. Then $A \in \mathscr{C}(M, \omega)$, condition $(F)$ is satisfied, and the corresponding space $E^{1}$ coincides with $\mathscr{D}\left((-A)^{1 / 2}\right)$.

Proposition 13 (see [27]). Let $A \in \mathscr{C}(M, 0)$, and let $E$ be a $U M D$ space. Then condition $(F)$ holds.

Theorem 14 (see [28]). Let $A$ and $\mathfrak{B}$ be operators satisfying condition (i) of Definition 9, and let $0 \in \rho(\mathfrak{B})$. The following conditions are equivalent:

(i) The $C_{0}$-cosine operator-valued function $C(\cdot, A)$ satisfies condition $(F)$. 
(ii) The operator $\mathfrak{B}$ generates a $C_{0}$-group $\exp (\cdot \mathfrak{B})$ on $E$.

(iii) The operator $\left(\begin{array}{cc}0 & \mathfrak{B} \\ \mathfrak{B} & 0\end{array}\right)$ with the domain $D(A) \times D(\mathfrak{B})$ generates a $C_{0}$-group on $E \times E$.

(iv) The operator $\mathscr{A}:=\left(\begin{array}{ll}0 & I \\ A & 0\end{array}\right)$ with the domain $D(A) \times D(\mathfrak{B})$ generates $C_{0}$-group $\exp (\cdot \mathscr{A})$ on $\mathscr{D}(\mathfrak{B}) \times E$, where $\mathscr{D}(\mathfrak{B})$ is the Banach space of elements $D(\mathfrak{B})$ endowed with the graph norm.

(v) The embedding $D(\mathfrak{B}) \subseteq E^{1}$ holds.

(vi) Consider $D(\mathfrak{B})=E^{1}$.

Proposition 15 (see [28]). Under the conditions of Theorem 14 , for $t \in \mathbb{R}$, one has

$$
\begin{aligned}
& \text { (i) } \exp (t \mathfrak{B})=C(t, A)+\mathfrak{B S}(t, A), \\
& C(t, A)=\frac{(\exp (t \mathfrak{B})+\exp (-t \mathfrak{B}))}{2}
\end{aligned}
$$

(ii) $\exp (t \mathscr{A})=\left(\begin{array}{cc}\mathfrak{B}^{-1} & 0 \\ 0 & I\end{array}\right) \exp \left(t\left(\begin{array}{cc}0 & \mathfrak{B} \\ \mathfrak{B} & 0\end{array}\right)\right)\left(\begin{array}{ll}\mathfrak{B} & 0 \\ 0 & I\end{array}\right)$.

Theorem 16 (see [29]). Let the operator $\mathfrak{B}=A^{1 / 2}$ in problem (7) have a bounded inverse $\mathfrak{B}^{-1} \in B(E)$ and be a generator of $a C_{0}$-group, and let the function $f(\cdot)$ have one of the following properties:

(i) $f(\cdot) \in C^{1}([0, T) ; E)$;

(ii) $\mathfrak{B} f(\cdot) \in C([0, T) ; E)$.

Then, for any $u^{0} \in D(A)$ and $u^{1} \in D(\mathfrak{B})$, there exists a unique classical solution of problem (7) given by formula (9) in the form

$$
\begin{aligned}
& u(t)= \frac{1}{2}(\exp (t \mathfrak{B})+\exp (-t \mathfrak{B})) u^{0} \\
&+\frac{1}{2}(\exp (t \mathfrak{B})-\exp (-t \mathfrak{B})) \mathfrak{B}^{-1} u^{1} \\
&+\frac{1}{2} \int_{0}^{t}(\exp ((t-s) \mathfrak{B})-\exp (-(t-s) \mathfrak{B})) \\
& \cdot \mathfrak{B}^{-1} f(s) d s, \quad t \in[0, T] .
\end{aligned}
$$

Denote $(S(\cdot, A) * f)(t):=\int_{0}^{t} S(t-s, A) f(s) d s, t \in[0, T]$.

Definition 17. One says that $C_{0}$-cosine operator function $C(\cdot, A)$ has the maximal regularity (MR-property) if $S(\cdot, A) *$ $f \in C^{2}([0, T] ; E)$ or, which is equivalent, $C(\cdot, A) * f \in$ $C([0, T] ; \mathscr{D}(A))$ for all $f(\cdot) \in C([0, T] ; E)$.

Definition 18. Let $\widetilde{E}$ be a Banach space being a subspace of the initial space $E$, and let $\Upsilon([0, T] ; E)$ be the Banach space of functions with values in $E$. Problem (7) is said to be coercively solvable in the pair of spaces $(\widetilde{E}, \Upsilon([0, T] ; E)$ ) (in other words, the solution $u(\cdot)$ has the maximal regularity property) if, for any right-hand side $f(\cdot) \in \Upsilon([0, T] ; E)$, there exists classical solution $u(\cdot)$ of Cauchy problem (7), for each $t$, the value of the solution $u(t)$ belongs to $\widetilde{E}$, and the following coercive inequality holds:

$$
\begin{aligned}
& \left\|u^{\prime \prime}(\cdot)\right\|_{\Upsilon([0, T] ; E)}+\|A u(\cdot)\|_{\Upsilon([0, T] ; E)} \\
& \quad \leq M\left(\|f(\cdot)\|_{\Upsilon([0, T] ; E)}+\left\|u^{0}\right\|_{\widetilde{E}}+\left\|u^{1}\right\|_{\widetilde{E}}\right) .
\end{aligned}
$$

Theorem 19 (see [30]). Let problem (7) be coercively solvable in the pair $(\mathscr{D}(A), C([0, T] ; E))$. Then $A \in B(E)$.

This result can also be reformulated as follows.

Theorem 20 (see [30]). The following statements are equivalent:

(i) For all $x, y \in D(A)$ and $f(\cdot) \in C([0, T] ; E)$, problem (7) has a classical solution.

(ii) The operator A generates a $C_{0}$-cosine operator function that satisfies the MR-property.

(iii) The operator A generates a $C_{0}$-cosine operator function that is of bounded semivariation on $[0, T]$.

(iv) $A$ is a bounded linear operator on $E$.

In the case of $L^{p}([0, T] ; E)$ space, the situation is the same.

Definition 21. The problem (7) is said to be coercively solvable in $L^{p}([0, T] ; E), 1 \leq p \leq \infty$, if, for any $f(\cdot) \in L^{p}([0, T] ; E)$, there exists a unique solution $u(\cdot)$ satisfying the equation almost everywhere such that $u(0)=u^{0}, u^{\prime}(0)=u^{1}, u^{\prime \prime}(\cdot)$, $A u(\cdot) \in L^{p}([0, T] ; E)$, and the following coercive inequality holds:

$$
\begin{aligned}
& \left\|u^{\prime \prime}(\cdot)\right\|_{L^{p}([0, T] ; E)}+\|A u(\cdot)\|_{L^{p}([0, T] ; E)} \\
& \quad \leq M(p)\left(\|f(\cdot)\|_{L^{p}([0, T] ; E)}+\left\|u^{0}\right\|_{D(A)}+\left\|u^{1}\right\|_{E^{1}}\right) .
\end{aligned}
$$

Theorem 22 (see [31]). Let problem (7) be coercively solvable in $L^{p}([0, T] ; E)$ with a certain $1 \leq p \leq \infty$. Then $A$ is bounded.

Well-posedness and maximal regularity for the problems

$$
\begin{aligned}
u^{\prime \prime}(t)+B(t) u^{\prime}(t)+A(t) u(t) & =f(t) \quad t \text { - a.e. } \\
u(0) & =u^{0}, u^{\prime}(0)=u^{1}, \\
u^{\prime \prime}(t)+A(t) u(t) & =f(t) \quad t-\text { a.e. } \\
u(0) & =u^{0}, u^{\prime}(0)=u^{1},
\end{aligned}
$$

were proved in case of Hilbert space $H$, where the operators $A(t)$ and $B(t)$ are associated with time-dependent sesquilinear forms with domain $V$ which is continuously imbedded into $H$ (see [32]). In Banach space $E$, problem (18) has been studied in $[33]$ in $L^{p}([0, T] ; E)$.

In $[34,35]$, they show existence, uniqueness, and maximal regularity of solution for the following differential equation with delay:

$$
\begin{aligned}
& u^{\prime \prime}(t)+B(t) u^{\prime}(t)+A(t) u(t) \\
& =G u_{t}^{\prime}(t)+F u_{t}(t)+f(t), \quad t \in \mathbb{R},
\end{aligned}
$$


where $f(\cdot) \in C^{\alpha}(\mathbb{R} ; E), 0<\alpha<1$. Here $F, G: C([-r, 0]$; $E) \rightarrow E$ are supposed to be bounded linear operators; $u_{t}(\cdot)=$ $u(t+\cdot)$ on $[-r, 0]$. Problem (20) with periodic conditions $u(0)=u(2 \pi), u^{\prime}(0)=u^{\prime}(2 \pi)$ was considered in [36].

Finally, in [37] they find that if the problem

$$
\begin{gathered}
u^{\prime \prime}(t)+B u^{\prime}(t)+A u(t)=f(t) \quad t \in[0, T] ; \\
u(0)=0, \quad u^{\prime}(0)=0
\end{gathered}
$$

has $L^{p}$-maximal regularity, where $1<p<\infty$, then the corresponding propagator of the sine type is an analytic function. The proof of this fact is based on the estimates of $\left\|\lambda\left(\lambda^{2} I+\lambda B+A\right)^{-1}\right\|$ and $\left\|B\left(\lambda^{2} I+\lambda B+A\right)^{-1}\right\|$ with $\operatorname{Re} \lambda>\omega$.

\section{The Weak Maximal Regularity (WMR-Property) Property}

As we saw in Section 2, maximal regularity for hyperbolic problem (7) in $C([0, T] ; E), L^{p}([0, T] ; E)$ spaces implies boundedness of the operator $A$. So in this occasion for unbounded operator $A$ it is very natural to give the following definition in case one would like to consider some kind of maximal regularity.

Definition 23 (see [38]). One says that $C_{0}$-cosine operator function $C(\cdot, A)$ has the weak maximal regularity (WMR-property) or the maximal regularity with loss (MRLproperty) if $S(\cdot, A) * f \in C^{2}([0, T] ; E)$ or, which is equivalent, $C(\cdot, A) * f \in C([0, T] ; \mathscr{D}(A))$ for all $f(\cdot) \in F([0, T] ; \widetilde{E}) \subset$ $C([0, T] ; E)$, where $\widetilde{E} \subset E$.

Definition 24. Let $\check{E}$ and $\widehat{E}$ be Banach spaces being subspaces of the original space $E$, and let $C([0, T] ; E)$ be the Banach space of continuous functions with values in $E$. Problem (7) is said to be weakly coercively solvable in the pair of spaces $((\check{E}, \widehat{E}), C([0, T] ; E))$ (in other words, the solution $u(\cdot)$ has the weak maximal regularity property) if, for any right-hand side $f(\cdot) \in F([0, T] ; E)$, there exists classical solution $u(\cdot)$ of Cauchy problem (7), for each $t$, the value of the solution $\left(u(t), u^{\prime}(t)\right)$ belongs to $\check{E} \times \widehat{E}$, and the following weak coercive inequality holds:

$$
\begin{aligned}
& \left\|u^{\prime \prime}(\cdot)\right\|_{C([0, T] ; E)}+\|A u(\cdot)\|_{C([0, T] ; E)} \\
& \quad \leq M\left(\|f(\cdot)\|_{F([0, T] ; E)}+\left\|u^{0}\right\|_{\check{E}}+\left\|u^{1}\right\|_{\widehat{E}}\right) .
\end{aligned}
$$

Remark 25. Note that in the cases $F([0, T] ; E)=C([0, T] ; E)$ and $\check{E}=D(A), \widehat{E}=D(\mathfrak{B})$ the weak maximal regularity (WMR-property) is equivalent to the maximal regularity in $C([0, T] ; E)$.

Theorem 26. Assume that condition $(F)$ is satisfied and $f(\cdot) \in$ $C^{1}([0, T] ; E) \cap C([0, T] ; D(\mathfrak{B}))$. Then problem (7) is weakly coercively solvable in the pair $((D(A), D(\mathfrak{B})), C([0, T] ; E))$ and the following estimate holds:

$$
\begin{gathered}
\left\|u^{\prime \prime}(\cdot)\right\|_{C([0, T] ; E)}+\|A u(\cdot)\|_{C([0, T] ; E)} \\
\leq M\left(\min \left(\|f(\cdot)\|_{C^{1}([0, T] ; E)},\|f(\cdot)\|_{C([0, T] ; D(\mathfrak{B}))}\right)\right. \\
\left.+\left\|A u^{0}\right\|_{E}+\left\|\mathfrak{B} u^{1}\right\|_{E}\right) .
\end{gathered}
$$

Proof. Following (15) from Theorem 16 one can write

$$
\begin{aligned}
A u(t)= & \frac{1}{2}(\exp (t \mathfrak{B})+\exp (-t \mathfrak{B})) A u^{0} \\
& +\frac{1}{2}(\exp (t \mathfrak{B})-\exp (-t \mathfrak{B})) \mathfrak{B} u^{1} \\
& +\frac{1}{2} \int_{0}^{t}(\exp ((t-s) \mathfrak{B})-\exp (-(t-s) \mathfrak{B})) \\
A u(t)= & \frac{1}{2}(\exp (t \mathfrak{B})+\exp (-t \mathfrak{B})) A u^{0} \\
& +\frac{1}{2}(\exp (t \mathfrak{B})-\exp (-t \mathfrak{B})) \mathfrak{B} u^{1}-f(t) \\
& +\frac{1}{2}(\exp (t \mathfrak{B})+\exp (-t \mathfrak{B})) f(0) \\
& +\frac{1}{2} \int_{0}^{t}(\exp ((t-s) \mathfrak{B})+\exp (-(t-s) \mathfrak{B})) \\
& \cdot f^{\prime}(s) d s, \quad t \in[0, T] .
\end{aligned}
$$

Using formulas (24) and (25), we get

$$
\begin{aligned}
\|A u(t)\|_{E} \leq & \frac{1}{2}(\|\exp (t \mathfrak{B})\|+\|\exp (-t \mathfrak{B})\|)\left\|A u^{0}\right\|_{E} \\
& +\frac{1}{2}(\|\exp (t \mathfrak{B})\|+\|\exp (-t \mathfrak{B})\|)\left\|\mathfrak{B} u^{1}\right\|_{E} \\
& +\frac{1}{2} \int_{0}^{t}(\|\exp ((t-s) \mathfrak{B})\| \\
& \quad+\|\exp (-(t-s) \mathfrak{B})\|)\|\mathfrak{B} f(s)\|_{E} d s \\
\leq & M\left(\|f(\cdot)\|_{C([0, T] ; D(\mathfrak{B}))}+\left\|A u^{0}\right\|_{E}+\left\|\mathfrak{B}^{1}\right\|_{E}\right),
\end{aligned}
$$


and also

$$
\begin{aligned}
\|A u(t)\|_{E} \leq & \frac{1}{2}(\|\exp (t \mathfrak{B})\|+\|\exp (-t \mathfrak{B})\|)\left\|A u^{0}\right\|_{E} \\
& +\frac{1}{2}(\|\exp (t \mathfrak{B})\|+\|\exp (-t \mathfrak{B})\|)\left\|\mathfrak{B} u^{1}\right\|_{E} \\
& +\frac{1}{2} \int_{0}^{t}(\|\exp ((t-s) \mathfrak{B})\|+\|\exp (-(t-s) \mathfrak{B})\|) \\
\cdot\left\|f^{\prime}(s)\right\|_{E} d s & \\
\leq & M\left(\left\|f^{\prime}(\cdot)\right\|_{C([0, T] ; E)}+\left\|A u^{0}\right\|_{E}+\left\|\mathfrak{B} u^{1}\right\|_{E}\right)
\end{aligned}
$$

for any $t \in[0, T]$. Then

$$
\begin{aligned}
& \|A u(\cdot)\|_{C([0, T] ; E)} \\
& \leq M\left(\min \left(\|f(\cdot)\|_{C^{1}([0, T] ; E)},\|f(\cdot)\|_{C([0, T] ; D(\mathfrak{B}))}\right)\right. \\
& \left.\quad+\left\|A u^{0}\right\|_{E}+\left\|\mathfrak{B} u^{1}\right\|_{E}\right) .
\end{aligned}
$$

By the triangle inequality, this last estimate and (7) yield

$$
\begin{aligned}
& \left\|u^{\prime \prime}(\cdot)\right\|_{C([0, T] ; E)} \\
& \leq M\left(\min \left(\|f(\cdot)\|_{C^{1}([0, T] ; E)},\|f(\cdot)\|_{C([0, T] ; D(\mathfrak{B}))}\right)\right. \\
& \left.\quad+\left\|A u^{0}\right\|_{E}+\left\|\mathfrak{B} u^{1}\right\|_{E}\right) .
\end{aligned}
$$

In a similar manner, one can show the following theorem.

Theorem 27. Assume that condition $(F)$ is satisfied and $f(\cdot) \in$ $W^{1, p}([0, T] ; E) \cap L^{p}([0, T] ; D(\mathfrak{B}))$. Then problem (7) is weakly coercively solvable in the pair $\left((D(A), D(\mathfrak{B})), L^{p}([0, T] ; E)\right)$ and the following estimate holds:

$$
\begin{aligned}
& \left\|u^{\prime \prime}(\cdot)\right\|_{L^{p}([0, T] ; E)}+\|A u(\cdot)\|_{L^{p}([0, T] ; E)} \\
& \leq M\left(\min \left(\|f(\cdot)\|_{W^{1, p}([0, T] ; E)},\|f(\cdot)\|_{L^{p}([0, T] ; D(\mathfrak{B}))}\right)\right. \\
& \left.+\left\|A u^{0}\right\|_{E}+\left\|\mathfrak{B} u^{1}\right\|_{E}\right) .
\end{aligned}
$$

Proof. Using identity (24), we get

$$
\begin{aligned}
A u(t)= & \frac{1}{2}(\exp (t \mathfrak{B})+\exp (-t \mathfrak{B})) A u^{0} \\
& +\frac{1}{2}(\exp (t \mathfrak{B})-\exp (-t \mathfrak{B})) \mathfrak{B} u^{1} \\
& +\frac{1}{2} \int_{0}^{T}(\exp (s \mathfrak{B})-\exp (-s \mathfrak{B})) \mathfrak{B} f^{*}(t-s) d s .
\end{aligned}
$$

Here

$$
f^{*}(t-s)= \begin{cases}f(t-s), & 0 \leq s \leq t, \\ 0, & t \leq s \leq T, t-s \notin[0, T] .\end{cases}
$$

From Minkowski's integral inequality, it follows that

$$
\begin{aligned}
& \|A u(\cdot)\|_{L^{p}([0, T] ; E)} \\
& \leq\left(\int_{0}^{T}\left(\frac{1}{2}(\|\exp (t \mathfrak{B})\|+\|\exp (-t \mathfrak{B})\|)\left\|A u^{0}\right\|_{E}\right)^{p} d t\right)^{1 / p} \\
& +\left(\int_{0}^{T}\left(\frac{1}{2}(\|\exp (t \mathfrak{B})\|+\|\exp (-t \mathfrak{B})\|)\left\|\mathfrak{B} u^{1}\right\|_{E}\right)^{p} d t\right)^{1 / p} \\
& +\int_{0}^{T} d s\left(\int _ { 0 } ^ { T } \left(\frac{1}{2}(\|\exp (s \mathfrak{B})\|+\|\exp (-s \mathfrak{B})\|)\right.\right. \\
& \left.\left.\quad \cdot\left\|f^{*}(t-s)\right\|_{E}\right)^{p} d t\right)^{1 / p} \\
& \leq M\left[\left\|A u^{0}\right\|_{E}+\left\|\mathfrak{B} u^{1}\right\|_{E}\right. \\
& +\int_{0}^{T} \frac{1}{2}(\|\exp (s \mathfrak{B})\|+\|\exp (-s \mathfrak{B})\|) d s \\
& \left.\quad\left(\int_{0}^{T}\left\|\mathfrak{B} f^{*}(t-s)\right\|_{E^{p}} d t\right)^{1 / p}\right] .
\end{aligned}
$$

Using the definition of the function $f^{*}(t-s)$, we get

$$
\left(\int_{0}^{T}\left\|\mathfrak{B} f^{*}(t-s)\right\|_{E^{p}} d t\right)^{1 / p} \leq\left(\int_{0}^{T}\|\mathfrak{B} f(t)\|_{E^{p}} d t\right)^{1 / p}
$$

Then

$$
\begin{aligned}
& \|A u(\cdot)\|_{L^{p}([0, T] ; E)} \\
& \quad \leq M\left[\left\|A u^{0}\right\|_{E}+\left\|\mathfrak{B} u^{1}\right\|_{E}+\left(\int_{0}^{T}\|\mathfrak{B} f(t)\|_{E^{p}} d t\right)^{1 / p}\right] \\
& \quad=M\left(\|f(\cdot)\|_{L^{p}([0, T] ; D(\mathfrak{B}))}+\left\|A u^{0}\right\|_{E}+\left\|\mathfrak{B} u^{1}\right\|_{E}\right) .
\end{aligned}
$$

Applying identity (25) and proceeding by analogy with estimate (35), we obtain

$$
\begin{aligned}
& \|A u(\cdot)\|_{L^{p}([0, T] ; E)} \\
& \quad \leq M\left(\|f(\cdot)\|_{W^{1, p}([0, T] ; E)}+\left\|A u^{0}\right\|_{E}+\left\|\mathfrak{B} u^{1}\right\|_{E}\right) .
\end{aligned}
$$

From the last two estimates, it follows that

\section{$\|A u(\cdot)\|_{L^{p}([0, T] ; E)}$}

$$
\begin{gathered}
\leq M\left(\min \left(\|f(\cdot)\|_{W^{1, p}([0, T] ; E)},\|f(\cdot)\|_{L^{p}([0, T] ; D(\mathfrak{B}))}\right)\right. \\
\left.+\left\|A u^{0}\right\|_{E}+\left\|\mathfrak{B} u^{1}\right\|_{E}\right)
\end{gathered}
$$


By the triangle inequality, this last estimate and (7) yield

$$
\begin{aligned}
& \left\|u^{\prime \prime}(\cdot)\right\|_{L^{p}([0, T] ; E)} \\
& \leq M\left(\min \left(\|f(\cdot)\|_{W^{1, p}([0, T] ; E)},\|f(\cdot)\|_{L^{p}([0, T] ; D(\mathfrak{B}))}\right)\right. \\
& \left.\quad+\left\|A u^{0}\right\|_{E}+\left\|\mathfrak{B} u^{1}\right\|_{E}\right) .
\end{aligned}
$$

3.1. Maximal Regularity in $C\left([0, T] ; E^{\theta}\right)$ and $C^{\alpha}([0, T] ; E)$ Spaces. As one can see from Section 2, there are open questions on maximal regularity in the area of maximal regularity for second order equation in the spaces like $C\left([0, T] ; E^{\theta}\right), C^{\alpha}([0, T] ; E), 0 \leq \alpha<1, L^{p}\left([0, T] ; E^{\theta}\right)$ spaces. The first order in time Cauchy problem (1) is not coercively well-posed in $C([0, T] ; E)$ space, but it is coercively wellposed in the spaces $C\left([0, T] ; E^{\theta}\right), C^{\alpha, 0}([0, T] ; E)$, where $E^{\theta}$ is interpolation space. In case of second order in time Cauchy problem (7), the strong maximal regularity property is independent of spaces and never holds.

The following relations for $C_{0}$-cosine operator function will be useful for us.

Proposition 28 (see $[28,39]$ ). For all $t, s \in \mathbb{R}$, one has the relations

(i) $C(t, A)=C(-t, A), S(-t, A)=-S(t, A), S(0, A)=0$;

(ii) $S(t+s, A)+S(t-s, A)=2 S(t, A) C(s, A)$;

(iii) $S(t+s, A)=S(t, A) C(s, A)+S(s, A) C(t, A)$;

(iv) $C(t+s, A)-C(t-s, A)=2 A S(t, A) S(s, A)$;

(v) $C(2 t, A)=2 C(t, A)^{2}-I, C(t, A)^{2}-A S(t, A)^{2}=I$.

Theorem 29. Let an operator $A$ be a generator of $C_{0}$-cosine operator function $C(t, A)$. Assume that Cauchy problem (7) is coercively well-posed in $C^{\alpha}([0, T] ; E)$ space. Then the operator $A$ is bounded.

Proof. Let us define the sequence of operators

$$
L_{\xi_{j}, t} x=\frac{1}{\xi_{j}^{\alpha}}\left(g\left(t+\xi_{j}\right)-g(t)\right) x, \quad \xi_{j} \longrightarrow 0, \text { as } j \longrightarrow \infty,
$$

where $g(t) x=A \int_{0}^{t} S(t-s, A) S(s, A) x$ for any $x \in E$. The function $g(\cdot)$ represents the function $A u(\cdot)$ which we have in (9) in case when $f(t)=S(t, A) x$. It is clear that such $f(\cdot) \in$ $C^{1}([0, T] ; E) \subset C^{\alpha}([0, T] ; E)$. If problem (7) is coercively well-posed in $C^{\alpha}([0, T] ; E)$ space, then $g(\cdot) \in C^{\alpha}([0, T] ; E)$ and, therefore, the sequence $\left\{L_{\xi_{j}, t} x\right\}_{j=1}^{\infty}$ is bounded for any $x \in E$. By the uniform boundedness principle, the norms $\left\|L_{\xi_{j}, t}\right\|$ are uniformly bounded as $j \rightarrow \infty$. In the meantime, $g(t)=S(t, A)-t C(t, A)$ and therefore $L_{\xi_{j}, t}=\left(1 / \xi_{j}^{\alpha}\right)(S(t+$ $\left.\left.\xi_{j}, A\right)-S(t, A)\right)-\left(1 / \xi_{j}^{\alpha}\right)\left(\left(t+\xi_{j}\right) C\left(t+\xi_{j}, A\right)-t C(t, A)\right)$. Hence, we get that $\left\|C\left(t+\xi_{j}, A\right)-C(t, A)\right\| \leq C \xi_{j}^{\alpha}$ for any $t \in(0, T)$ and $\xi_{j} \rightarrow 0$. This implies that the operator $A$ must be bounded (see [6]).
Theorem 30. Let an operator $A$ be a generator of $C_{0}$-cosine operator function $C(t, A)$. Assume that Cauchy problem (7) is coercively well-posed in $C\left([0, T] ; E^{\theta}\right)$ space. Then the operator $A$ is bounded.

Proof. We consider the function $g(t) x=A \int_{0}^{t} S(t-s$, A) $C(s, A)(-A)^{-\alpha} x d s$. For any $x \in E$, the function $f(s)=C(s$, $A)(-A)^{-\alpha} x$ belongs to $C\left([0, T] ; E^{\alpha}\right)$ space and therefore well-posedness in $C\left([0, T] ; E^{\alpha}\right)$ implies that $g(\cdot) \in C([0$, $\left.T] ; E^{\alpha}\right)$. Then integrating $A \int_{0}^{t} S(t-s, A) C(s, A)(-A)^{-\alpha} x d s$ by simple calculations which use (ii) from Proposition 28, one can find that $\|t A S(t, A)\| \leq$ constant. So for any $t>0$ one gets by (iv) from Proposition 28 that

$$
\begin{aligned}
& \|C(t+h, A)-C(t-h, A)\| \\
& \quad \leq\|2 A S(t, A)\|\|S(h, A)\| \longrightarrow 0,
\end{aligned}
$$

as $h \rightarrow 0$, and therefore $A$ is bounded.

Theorem 31. Assume that condition $(F)$ is satisfied and $f(\cdot) \in$ $C^{1}\left([0, T] ; E^{\theta}\right) \cap C\left([0, T] ; D\left(\mathfrak{B}^{1+2 \theta}\right)\right)$. Then problem (7) is weakly coercively solvable in the pair $\left(\left(D\left(A^{1+\theta}\right), D\left(\mathfrak{B}^{1+2 \theta}\right)\right)\right.$, $\left.C\left([0, T] ; E^{\theta}\right)\right)$ and the following estimate holds:

$$
\begin{gathered}
\left\|u^{\prime \prime}(\cdot)\right\|_{C\left([0, T] ; E^{\theta}\right)}+\|A u(\cdot)\|_{C\left([0, T] ; E^{\theta}\right)} \\
\leq M\left(\min \left(\|f(\cdot)\|_{C^{1}\left([0, T] ; E^{\theta}\right)},\|f(\cdot)\|_{C\left([0, T] ; D\left(\mathfrak{B}^{1+2 \theta}\right)\right)}\right)\right. \\
\left.+\left\|A u^{0}\right\|_{E^{\theta}}+\left\|\mathfrak{B} u^{1}\right\|_{E^{\theta}}\right) .
\end{gathered}
$$

Proof. From (15) of Theorem 16, one can write

$$
\begin{aligned}
A u(t)= & \frac{1}{2}(\exp (t \mathfrak{B})+\exp (-t \mathfrak{B})) A u^{0} \\
& +\frac{1}{2}(\exp (t \mathfrak{B})-\exp (-t \mathfrak{B})) \mathfrak{B} u^{1} \\
& +\frac{1}{2} \int_{0}^{t}(\exp ((t-s) \mathfrak{B})-\exp (-(t-s) \mathfrak{B})) \\
& \cdot \mathfrak{B} f(s) d s, \quad t \in[0, T] .
\end{aligned}
$$

Integrating by parts and applying the operator $A^{\theta}$ also to (42), one gets

$$
\begin{aligned}
& \|A u(\cdot)\|_{C\left([0, T] ; E^{\theta}\right)} \\
& \leq C\left(\left\|A u^{0}\right\|_{E^{\theta}}+\left\|\mathfrak{B} u^{1}\right\|_{E^{\theta}}\right. \\
& \left.\quad+\min \left(\|\mathfrak{B} f(\cdot)\|_{C\left([0, T] ; E^{\theta}\right)},\|f(\cdot)\|_{C^{1}\left([0, T] ; E^{\theta}\right)}\right)\right) .
\end{aligned}
$$

Now, we consider the weak coercive solvability of problem (7) in the Banach space $C^{\alpha}([0, T] ; E)(0<\alpha<1)$, obtained by completion of the set of smooth $E$-valued functions $\varphi(t)$ on $[0, T]$ in the norm

$$
\begin{aligned}
\|\varphi\|_{C^{\alpha}([0, T] ; E)}= & \max _{0 \leq t \leq T}\|\varphi(t)\|_{E} \\
& +\sup _{0 \leq t<t+\tau \leq T} \frac{\|\varphi(t+\tau)-\varphi(t)\|_{E}}{\tau^{\beta}} .
\end{aligned}
$$


Theorem 32. Assume that condition $(F)$ is satisfied and $f(\cdot) \in$ $C^{1+\alpha}\left([0, T] ; D\left(B^{\alpha}\right)\right) \cap C^{\alpha}\left([0, T] ; D\left(B^{1+\alpha}\right)\right)$. Then problem (7) is weakly coercively solvable in the pair $\left(\left(D\left(A^{1+\alpha / 2}\right)\right.\right.$, $\left.\left.D\left(\mathfrak{B}^{1+\alpha}\right)\right), C^{\alpha}([0, T] ; E)\right)$ and the following estimate holds:

$$
\begin{gathered}
\left\|u^{\prime \prime}(\cdot)\right\|_{C^{\alpha}([0, T] ; E)}+\|A u(\cdot)\|_{C^{\alpha}([0, T] ; E)} \\
\leq M\left(\min \left(\|f(\cdot)\|_{C^{1+\alpha}\left([0, T] ; D\left(\mathfrak{B}^{\alpha}\right)\right)},\|f(\cdot)\|_{C^{\alpha}\left([0, T] ; D\left(\mathfrak{B}^{1+\alpha}\right)\right)}\right)\right. \\
\left.+\left\|A^{1+\alpha / 2} u^{0}\right\|_{E}+\left\|\mathfrak{B}^{1+\alpha} u^{1}\right\|_{E}\right) .
\end{gathered}
$$

Proof. By Theorem 14,

$$
\begin{aligned}
& \|A u(\cdot)\|_{C([0, T] ; E)} \\
& \leq M\left(\min \left(\|f(\cdot)\|_{C^{1}([0, T] ; E)},\|f(\cdot)\|_{C([0, T] ; D(\mathfrak{B}))}\right)\right. \\
& \left.\quad+\left\|A u^{0}\right\|_{E}+\left\|\mathfrak{B} u^{1}\right\|_{E}\right) .
\end{aligned}
$$

Then, from the definition of the space $C^{\alpha}([0, T] ; E)$ and the estimate $\left\|B^{-\alpha}\right\| \leq M$, it follows that

$$
\begin{aligned}
& \|A u(\cdot)\|_{C([0, T] ; E)} \\
& \leq M_{1}\left(\min \left(\|f(\cdot)\|_{C^{1+\alpha}([0, T] ; E)},\|f(\cdot)\|_{C^{\alpha}([0, T] ; D(\mathfrak{B}))}\right)\right. \\
& \left.\quad+\left\|A^{1+\alpha / 2} u^{0}\right\|_{E}+\left\|\mathfrak{B}^{1+\alpha} u^{1}\right\|_{E}\right) .
\end{aligned}
$$

Now, let us estimate the difference $A u(t+\tau)-A u(t)$ for $0 \leq$ $t<t+\tau \leq T$. From identity (24), it follows that

$$
\begin{aligned}
A u(t+\tau)-A u(t) & \\
= & \frac{1}{2}(\exp ((t+\tau) \mathfrak{B})-\exp (t \mathfrak{B}) \\
& +\exp (-(t+\tau) \mathfrak{B})-\exp (-t \mathfrak{B})) A u^{0} \\
& +\frac{1}{2}(\exp ((t+\tau) \mathfrak{B})-\exp (t \mathfrak{B}) \\
& -\exp (-(t+\tau) \mathfrak{B})+\exp (-t \mathfrak{B})) \mathfrak{B} u^{1} \\
+\frac{1}{2} \int_{0}^{\tau}(\exp ((t+\tau-s) \mathfrak{B}) & -\exp (-(t+\tau-s) \mathfrak{B})) \mathfrak{B} f(s) d s \\
+ & \frac{1}{2} \int_{0}^{t}(\exp (s \mathfrak{B})-\exp (-s \mathfrak{B})) \\
\cdot & \mathfrak{B}(f(t+\tau-s)-f(t-s)) d s .
\end{aligned}
$$

Using the last identity, we get

$$
\begin{gathered}
\|A u(t+\tau)-A u(t)\|_{E} \\
\leq \frac{1}{2}\left(\left\|(\exp ((t+\tau) \mathfrak{B})-\exp (t \mathfrak{B})) A u^{0}\right\|_{E}\right. \\
\left.+\left\|(\exp (-(t+\tau) \mathfrak{B})-\exp (-t \mathfrak{B})) A u^{0}\right\|_{E}\right) \\
+\frac{1}{2}\left(\left\|(\exp ((t+\tau) \mathfrak{B})-\exp (t \mathfrak{B})) \mathfrak{B} u^{1}\right\|_{E}\right. \\
\left.+\left\|(\exp (-(t+\tau) \mathfrak{B})-\exp (-t \mathfrak{B})) \mathfrak{B} u^{1}\right\|_{E}\right) \\
+\frac{1}{2} \int_{0}^{\tau}(\|\exp ((t+\tau-s) \mathfrak{B})\| \\
+\|\exp (-(t+\tau-s) \mathfrak{B})\|)\|\mathfrak{B} f(s)\|_{E} d s \\
+\frac{1}{2} \int_{0}^{t}(\|\exp (s \mathfrak{B})\|+\|\exp (-s \mathfrak{B})\|) \\
\cdot\|\mathfrak{B}(f(t+\tau-s)-f(t-s))\|_{E} d s \\
\leq M \tau^{\alpha}\left(\|f(\cdot)\|_{C^{\alpha}([0, T] ; D(\mathfrak{B}))}\right. \\
\left.+\left\|A^{1+\alpha / 2} u^{0}\right\|_{E}+\left\|\mathfrak{B}^{1+\alpha} u^{1}\right\|_{E}\right) .
\end{gathered}
$$

Estimates (47) and (49) give

$$
\begin{aligned}
& \|A u(\cdot)\|_{C^{\alpha}([0, T] ; E)} \\
& \quad \leq M\left(\|f(\cdot)\|_{C^{\alpha}([0, T] ; D(\mathfrak{B}))}\right. \\
& \left.\quad+\left\|A^{1+\alpha / 2} u^{0}\right\|_{E}+\left\|\mathfrak{B}^{1+\alpha} u^{1}\right\|_{E}\right) .
\end{aligned}
$$

Applying identity (25) and proceeding in a similar way as in estimate (49), we obtain

$$
\begin{aligned}
& \|A u(\cdot)\|_{C^{\alpha}([0, T] ; E)} \\
& \quad \leq M\left(\|f(\cdot)\|_{C^{1+\alpha}([0, T] ; E)}\right. \\
& \left.\quad+\left\|A^{1+\alpha / 2} u^{0}\right\|_{E}+\left\|\mathfrak{B}^{1+\alpha} u^{1}\right\|_{E}\right) .
\end{aligned}
$$

From the last two estimates, it follows that

$$
\begin{aligned}
& \|A u(\cdot)\|_{C^{\alpha}([0, T] ; E)} \\
& \leq M\left(\min \left(\|f(\cdot)\|_{C^{1+\alpha}([0, T] ; E)},\|f(\cdot)\|_{C^{\alpha}([0, T] ; D(\mathfrak{B}))}\right)\right. \\
& \left.\quad+\left\|A^{1+\alpha / 2} u^{0}\right\|_{E}+\left\|\mathfrak{B}^{1+\alpha} u^{1}\right\|_{E}\right) .
\end{aligned}
$$

By the triangle inequality, this last estimate and (7) yield

$$
\begin{aligned}
& \left\|u^{\prime \prime}(\cdot)\right\|_{C^{\alpha}([0, T] ; E)} \\
& \leq M\left(\min \left(\|f(\cdot)\|_{C^{1+\alpha}([0, T] ; E)},\|f(\cdot)\|_{C^{\alpha}([0, T] ; D(\mathfrak{B}))}\right)\right. \\
& \left.\quad+\left\|A^{1+\alpha / 2} u^{0}\right\|_{E}+\left\|\mathfrak{B}^{1+\alpha} u^{1}\right\|_{E}\right) .
\end{aligned}
$$


Remark 33. As was mentioned in Theorem 22, the coercive well-posedness of $(7)$ in $L^{P}([0, T] ; E)$ spaces holds in general if and only if $A$ is bounded. Even in case of periodic functions spaces $L_{2 \pi}^{p}(\mathbb{R} ; E), C_{2 \pi}^{\alpha}(\mathbb{R} ; E)$, the situation is not changed: in [10], it was shown that maximal regularity holds if and only if $\left\{-k^{2}: k \in \mathbb{Z}\right\} \subset \rho(A)$, $\sup _{k \in \mathbb{Z}}\left\|k\left(-k^{2} I-A\right)^{-1}\right\|<$ $\infty$, which does not hold for general bounded $C_{0}$-cosine operator function in Hilbert space $E$. In the particular case of UMD spaces, $E$ maximal regularity of Cauchy problem (7) for second order in time differential equations is defined by location of the spectrum of operator $A$, but not by smoothness of the space $E$; that is, the function $\lambda^{2}\left(\lambda^{2} I-A\right)^{-1}$ must be Fourier multiplier which is not true in general. Thus there is no coercive well-posedness of $(7)$ in $L^{p}\left([0, T] ; E^{\theta}\right)$ space in general.

Applying $A^{\theta}$ to formulas (24) and (25) and proceeding similarly to Theorem 16, we obtain the following theorem.

Theorem 34. Assume that condition $(F)$ is satisfied and $f(\cdot) \in$ $W^{1, p}\left([0, T] ; E^{\theta}\right) \cap L^{p}\left([0, T] ; D\left(\mathfrak{B}^{1+2 \theta}\right)\right)$. Then problem (7) is weakly coercively solvable in the pair $\left(\left(D\left(A^{1+2 \theta}\right)\right.\right.$, $\left.\left.D\left(\mathfrak{B}^{1+2 \theta}\right)\right), L^{p}\left([0, T] ; E^{\theta}\right)\right)$ and the following estimate holds:

$$
\begin{gathered}
\left\|u^{\prime \prime}(\cdot)\right\|_{L^{p}\left([0, T] ; E^{\theta}\right)}+\|A u(\cdot)\|_{L^{p}\left([0, T] ; E^{\theta}\right)} \\
\leq M\left(\min \left(\|f(\cdot)\|_{W^{1, p}\left([0, T] ; E^{\theta}\right)},\|f(\cdot)\|_{L^{p}\left([0, T] ; D\left(\mathfrak{B}^{1+2 \theta}\right)\right)}\right)\right. \\
\left.\quad+\left\|A u^{0}\right\|_{E^{\theta}}+\left\|\mathfrak{B} u^{1}\right\|_{E^{\theta}}\right)
\end{gathered}
$$

\section{Elliptic Equations}

In [2] the coercive well-posedness in $L^{p}([0, T] ; E)$ of the problem

$$
\begin{gathered}
u^{\prime \prime}(t)=A u(t)+f(t), \quad t \in[0, T], \\
u(0)=u^{0}, \quad u(T)=u^{T}
\end{gathered}
$$

has been considered under condition of positivity of the operator $A$. In such case, the operator $-A^{1 / 2}$ generates an analytic $C_{0}$-semigroup.

In Banach space $E$, we consider boundary value problem (55) with positive operator $A$ and $f(\cdot)$ is some function from some function space. Problem (55) can be considered in different functional spaces. Function $u(\cdot)$ is called a solution in classical sense of problem (55) if the following conditions are satisfied:

(i) $u(\cdot)$ is twice continuously differentiable on the interval $[0, T]$. The derivative at the endpoints of the segment are understood as the appropriate unilateral derivatives.

(ii) The element $u(t)$ belongs to $D(A)$ for all $t \in[0, T]$, and the function $A u(\cdot)$ is continuous on the interval $[0, T]$. (iii) $u(\cdot)$ satisfies the equation and boundary conditions (55).

Let $C([0, T] ; E)$ be the space of all continuous functions $\varphi(\cdot)$ defined on $[0, T]$ with values in $E$ equipped with the norm $\|\varphi(\cdot)\|_{C([0, T] ; E)}=\max _{0 \leq t \leq T}\|\varphi(t)\|_{E}$. The coercive wellposedness in $C([0, T] ; E)$ of boundary value problem (55) means that for the solution $u(\cdot) \in C([0, T] ; E)$ the coercive inequality

$$
\begin{aligned}
& \left\|u^{\prime \prime}(\cdot)\right\|_{C([0, T] ; E)}+\|A u(\cdot)\|_{C([0, T] ; E)} \\
& \quad \leq M\left(\|f(\cdot)\|_{C([0, T] ; E)}+\left\|A u^{0}\right\|_{E}+\left\|A u^{T}\right\|_{E}\right)
\end{aligned}
$$

holds with some constant $M$, which is independent of $u^{0}, u^{T}, f(\cdot) \in C([0, T] ; E)$. It turns out that this positivity property of the operator $A$ in $E$ is a necessary condition of well-posedness of boundary value problem (55) in $C([0, T] ; E)[2]$. One can ask: does the positivity of the operator $A$ in $E$ imply the well-posedness of boundary value problem (55)? In the general case, the answer is no (see [2]), so the coercive inequality does not take place in $C([0, T] ; E)$ for boundary value problem (55).

We recall that if Cauchy problem (1) is coercively wellposed in the space $C([0, T] ; E)$, then operator $A$ has to be bounded or the space $E$ contains a subspace isomorphic to $c_{0}$ (see [15]). One gets a similar situation for problem (55).

Theorem 35 (see [12]). Let $A$ be a positive operator on E. Assume that problem (55) is coercively well-posed in the space $C([0, T] ; E)$. Then either $A$ is bounded or E contains a closed subspace which is isomorphic to $c_{0}$.

Consider $C^{\beta, \gamma}([0, T] ; E), 0 \leq \gamma \leq \beta, 0<\beta<1$, the Banach space obtained by completion of the set of smooth $E$-valued functions $\varphi(\cdot)$ on $[0, T]$ in the norm

$$
\begin{aligned}
\|\varphi(\cdot)\|_{C^{\beta, \gamma}([0, T] ; E)} & \\
= & \max _{0 \leq t \leq T}\|\varphi(t)\|_{E} \\
& +\sup _{0 \leq t<t+\tau \leq T} \frac{(t+\tau)^{\gamma}(T-t)^{\gamma}\|\varphi(t+\tau)-\varphi(t)\|_{E}}{\tau^{\beta}} .
\end{aligned}
$$

Theorem 36 (see $[2,12])$. Let $A$ be a positive operator in Banach space $E$ and $f(\cdot) \in C^{\beta, \gamma}([0, T] ; E), 0 \leq \gamma \leq \beta$, $0<\beta<1$. Then the solution of boundary value problem (55) belongs to $u(\cdot) \in C^{\beta, \gamma}([0, T] ; E)$ and the following coercive inequalities hold:

$$
\begin{gathered}
\left\|u^{\prime \prime}(\cdot)\right\|_{C\left([0, T] ; E_{\beta-\gamma}\right)} \\
\leq M\left(\left\|A u^{0}+f(0)\right\|_{E_{\beta-\gamma}}+\left\|A u^{T}+f(T)\right\|_{E_{\beta-\gamma}}\right. \\
\left.+\beta^{-1}(1-\beta)^{-1}\|f\|_{C^{\beta, \gamma}([0, T] ; E)}\right)
\end{gathered}
$$




$$
\begin{aligned}
& \text { for } A u^{0}+f(0) \in E_{\beta-\gamma}, A u^{T}+f(T) \in E_{\beta-\gamma}, \\
& \qquad u^{\prime \prime}(\cdot)\left\|_{C^{\beta, \gamma}([0, T] ; E)}+\right\| A u(\cdot) \|_{C^{\beta, \gamma}([0, T] ; E)} \\
& \leq M\left(\left|A u^{0}+f(0)\right|_{0}^{\beta, \gamma}+\left|A u^{T}+f(T)\right|_{0}^{\beta, \gamma}\right. \\
& \left.+\beta^{-1}(1-\beta)^{-1}\|f(\cdot)\|_{C^{\beta, \gamma}([0, T] ; E)}\right)
\end{aligned}
$$

for $A u^{0}+f(0) \in E_{0}^{\beta, \gamma}, A u^{T}+f(T) \in E_{0}^{\beta, \gamma}$, where $M$ is independent of $\beta, \gamma, u^{0}, u^{T}$, and $f(\cdot)$. Here, $|w|_{0}^{\beta, \gamma}$ denotes the norm of Banach space $E_{0}^{\beta, \gamma}$, which consists of those $w \in E$ for which the norm

$$
\begin{aligned}
&|w|_{0}^{\beta, \gamma}= \max _{0 \leq z \leq T}\left\|e^{-z A^{1 / 2}} w\right\|_{E} \\
&+\sup _{0 \leq z<z+\tau \leq T} \tau^{-\beta}(z+\tau)^{\gamma}(T-z)^{\gamma} \\
& \cdot\left\|\left(e^{-(z+\tau) A^{1 / 2}}-e^{-z A^{1 / 2}}\right) w\right\|_{E}
\end{aligned}
$$

is finite and the Banach space $E_{\alpha}=E_{\alpha}\left(A^{1 / 2}, E\right), 0<\alpha<$ 1 , consists of those $v \in E$ for which the norm $\|v\|_{E_{\alpha}}=$ $\sup _{z>0} z^{1-\alpha}\left\|A^{1 / 2} \exp \left(-z A^{1 / 2}\right) v\right\|_{E}+\|v\|_{E}$ is finite.

Consider $C^{\beta, \gamma}\left([0, T], E_{\alpha-\beta}\right), 0 \leq \gamma \leq \beta \leq \alpha, 0<\alpha<1$. To these, there correspond the spaces of traces $E_{\alpha-\beta}^{\beta, \gamma}$, which consist of elements $w \in E$ for which the norm

$$
\begin{aligned}
&|w|_{\alpha-\beta}^{\beta, \gamma}= \max _{0 \leq z \leq 1}\left\|e^{-z A^{1 / 2}} w\right\|_{E_{\alpha-\beta}} \\
&+\sup _{0 \leq z<z+\tau \leq 1} \tau^{-\beta}(z+\tau)^{\gamma}(1-z)^{\gamma} \\
& \cdot\left\|\left(e^{-(z+\tau) A^{1 / 2}}-e^{-z A^{1 / 2}}\right) w\right\|_{E_{\alpha-\beta}}
\end{aligned}
$$

is finite.

Theorem 37 (see [12]). Let $A$ be a positive operator in Banach space $E, A u^{0}+f(0) \in E_{\alpha-\beta}^{\beta, \gamma}, A u^{T}+f(T) \in E_{\alpha-\beta}^{\beta, \gamma}$ and $f(\cdot) \in$ $C^{\beta, \gamma}\left([0, T] ; E_{\alpha-\beta}\right), 0 \leq \gamma \leq \beta \leq \alpha, 0<\alpha<1$. Then for the solution $u(\cdot)$ in $C^{\beta, \gamma}\left([0, T] ; E_{\alpha-\beta}\right)$ of boundary value problem (55) the coercive inequality

$$
\begin{array}{r}
\left\|u^{\prime \prime}(\cdot)\right\|_{C^{\beta, \gamma}\left([0, T] ; E_{\alpha-\beta}\right)}+\|A u(\cdot)\|_{C^{\beta, \gamma}\left([0, T] ; E_{\alpha-\beta}\right)} \\
+\left\|u^{\prime \prime}(\cdot)\right\|_{C\left([0, T] ; E_{\alpha-\beta}^{\beta, \gamma}\right)} \\
\leq M\left(\left|A u^{0}+f(0)\right|_{\alpha-\beta}^{\beta, \gamma}+\left|A u^{T}+f(T)\right|_{\alpha-\beta}^{\beta, \gamma}\right. \\
\left.+\alpha^{-1}(1-\alpha)^{-1}\|f(\cdot)\|_{C^{\beta, \gamma}\left([0, T] ; E_{\alpha-\beta}\right)}\right)
\end{array}
$$

holds, where $M$ is independent of $\alpha, \beta, \gamma, u^{0}, u^{T}$, and $f(\cdot)$.
Theorem 38 (see [12]). Let $A$ be a positive operator in Banach space $E, A u^{0}+f(0) \in E_{\alpha-\gamma}, A u^{T}+f(T) \in E_{\alpha-\beta}$, and $f(\cdot) \epsilon$ $C^{\beta, \gamma}\left([0, T] ; E_{\alpha-\gamma}\right), 0 \leq \gamma \leq \beta \leq \alpha, 0<\alpha<1$. Then for the solution $u(\cdot)$ in $C^{\beta, \gamma}\left([0, T] ; E_{\alpha-\beta}\right)$ of boundary value problem (55) the coercive inequality

$$
\begin{aligned}
\| u^{\prime \prime}(\cdot) & \left\|_{C^{\beta, \gamma}\left([0, T] ; E_{\alpha-\beta}\right)}+\right\| A u(\cdot) \|_{C^{\beta, \gamma}\left([0, T] ; E_{\alpha-\beta}\right)} \\
+ & \left\|u^{\prime \prime}(\cdot)\right\|_{C\left([0, T] ; E_{\alpha-\gamma}\right)} \\
\leq & M \alpha^{-1}(1-\alpha)^{-1} \\
& \cdot\left(\left\|A u^{0}+f(0)\right\|_{E_{\alpha-\gamma}}\right. \\
& \left.+\left\|A u^{T}+f(T)\right\|_{E_{\alpha-\gamma}}+\|f(\cdot)\|_{C^{\beta, \gamma}\left([0, T] ; E_{\alpha-\beta}\right)}\right)
\end{aligned}
$$

holds, where $M$ is independent of $\alpha, \beta, \gamma, u^{0}, u^{T}$, and $f(\cdot)$.

Let us consider problem $(55)$ in the spaces $L^{p}([0, T] ; E)$, $1 \leq p<\infty$, of all strongly measurable $E$-valued functions $v(\cdot)$ on $[0, T]$ with the norm $\|v\|_{L^{p}([0, T] ; E)}=\left(\int_{0}^{T}\|v(t)\|_{E}^{p} d t\right)^{1 / p}$. Function $v(\cdot)$ is said to be absolutely continuous if it has a derivative $v^{\prime}(t)$ for almost every $t$ such that $v^{\prime}(\cdot) \in$ $L^{1}([0, T] ; E)$ and if the Newton-Leibniz formula $v(t)-v(\tau)=$ $\int_{\tau}^{t} v^{\prime}(s) d s$ holds for all $t, \tau \in[0, T]$. Here the integral is understood in the sense of Bochner. Function $u(\cdot)$ is said to be a solution of problem (55) in $L^{p}([0, T] ; E)$ if it is absolutely continuous, the functions $u^{\prime \prime}(\cdot)$ and $A u(\cdot)$ belong to $L^{p}([0, T] ; E),(55)$ is satisfied for almost every $t$, and $u(0)=$ $u^{0}, u(T)=u^{T}$. From this definition, it follows that a necessary condition for the solvability of problem (55) in $L^{p}([0, T] ; E)$ is that $f(\cdot) \in L^{p}([0, T] ; E)$. One can show that in certain cases this condition is also sufficient for the solvability of problem (55). Concerning the boundary elements, in contrast to the situation considered earlier, from the solvability of problem (55) in $L^{p}([0, T] ; E)$, it follows only that $u^{0}, u^{T} \in E$. From the unique solvability of (55), it follows that the operator $u\left(t ; f(t), u^{0}, u^{T}\right)$ is bounded in $L^{p}([0, T] ; E)$ and one has the coercive inequality

$$
\begin{aligned}
& \left\|u^{\prime \prime}\right\|_{L^{p}([0, T] ; E)}+\|A u(t)\|_{L^{p}([0, T] ; E)} \\
& \quad \leq M_{C}\left(\|f(\cdot)\|_{L^{p}([0, T] ; E)}+\left\|A u^{0}\right\|_{E}+\left\|A u^{T}\right\|_{E}\right),
\end{aligned}
$$

where $1 \leq M_{C}<+\infty$ is independent of $u^{0}, u^{T}$, and $f(\cdot)$. From that, one can obtain the positivity of $A$ under the stronger assumption that the operator $A^{-1}$ is compact in $E$ (see [40]).

Theorem 39 (see [40]). Let $A$ be a positive operator in a Banach space E. Suppose problem (55) is coercively well-posed in $L^{p_{0}}([0, T] ; E)$ for some $1<p_{0}<\infty$. Then it is coercively 
well-posed in $L^{p}([0, T] ; E)$ for any $1<p<\infty$ and the coercivity inequality holds:

$$
\begin{gathered}
\left\|u^{\prime \prime}(\cdot)\right\|_{L^{p}([0, T], E)}+\|A u(\cdot)\|_{L^{p}([0, T], E)} \\
+\|u(\cdot)\|_{C\left([0, T] ; E_{1-1 / p, p}\left(A^{1 / 2}, D\left(A^{1 / 2}\right)\right)\right)} \\
\leq \frac{M\left(p_{0}\right) p^{2}}{p-1}\|f(\cdot)\|_{L^{p}([0, T] ; E)} \\
+M\left(\left\|u^{0}\right\|_{E_{1-1 / p, p}\left(A^{1 / 2}, D\left(A^{1 / 2}\right)\right)}\right. \\
\left.\quad+\left\|u^{T}\right\|_{E_{1-1 / p, p}\left(A^{1 / 2}, D\left(A^{1 / 2}\right)\right)}\right),
\end{gathered}
$$

where $M\left(p_{0}\right)$ and $M$ are independent of $p, u^{0}, u^{T}$, and $f(\cdot)$.

Theorem 40 (see [41]). Let $1<p<\infty$ and $0<\alpha<1$. Suppose that $A$ is a positive operator in Banach space E. Then problem (55) is coercively well-posed in $L^{p}\left([0, T] ; E_{\alpha, p}\right)$ and the coercivity inequality holds:

$$
\begin{gathered}
\left\|u^{\prime \prime}(\cdot)\right\|_{L^{p}\left([0, T] ; E_{\alpha, p}\right)}+\|A u(\cdot)\|_{L^{p}\left([0, T] ; E_{\alpha, p}\right)} \\
\leq \frac{M}{\alpha(1-\alpha)}\|f(\cdot)\|_{L^{p}\left([0, T] ; E_{\alpha, p}\right)} \\
\quad+M\left(\left\|A u^{0}\right\|_{E_{\alpha, p}}+\left\|A u^{T}\right\|_{E_{\alpha, p}}\right)
\end{gathered}
$$

where $M$ is independent of $\alpha, p, u^{0}, u^{T}$, and $f(\cdot)$.

From these theorems we have the following.

Theorem 41. Let $1<p, q<\infty$ and $0<\alpha<1$. Suppose that $A$ is a positive operator in Banach space E. Then problem (55) is coercively well-posed in $L^{p}\left([0, T] ; E_{\alpha, q}\right)$ and the coercivity inequality holds:

$$
\begin{gathered}
\left\|u^{\prime \prime}(\cdot)\right\|_{L^{p}\left([0, T], E_{\alpha, q}\right)}+\|A u(\cdot)\|_{L^{p}\left([0, T] ; E_{\alpha, q}\right)} \\
\leq \frac{M(q)}{\alpha(1-\alpha)}\|f(\cdot)\|_{L^{p}\left([0, T] ; E_{\alpha, q}\right)} \\
\quad+M\left(\left\|A u^{0}\right\|_{E_{\alpha, q}}+\left\|A u^{T}\right\|_{E_{\alpha, q}}\right)
\end{gathered}
$$

where $M(q)$ and $M$ are independent of $\alpha, p, u^{0}, u^{T}$, and $f(\cdot)$. Here the Banach space $E_{\alpha, q}=E_{\alpha, q}\left(A^{1 / 2}, E\right), 0<\alpha<1,1<$ $q<\infty$, consists of those $v \in E$ for which the norm

$$
\|v\|_{E_{\alpha, q}}=\left(\int_{0}^{\infty} \lambda^{1-\alpha}\left\|A^{1 / 2} \exp \left(-\lambda A^{1 / 2}\right) v\right\|_{E}^{q} \frac{d \lambda}{\lambda}\right)^{1 / q}
$$

is finite.

In $[42,43]$, abstract elliptic differential equation (55) with Dirichlet-Neumann boundary conditions

$$
u(0)=d_{0}, \quad u^{\prime}(1)=n_{1}
$$

was considered. Maximal regularity in $C^{\alpha}([0,1] ; E)$ space is obtained if $d_{0} \in D(A), n_{1} \in D\left(A^{1 / 2}\right)$.

\section{General Approximation Scheme}

The general approximation scheme, due to [44-47], can be described in the following way (see also [12]). Let $E_{n}$ and $E$ be Banach spaces and let $\left\{p_{n}\right\}$ be a sequence of linear bounded operators $p_{n}: E \rightarrow E_{n}, p_{n} \in B\left(E, E_{n}\right), n \in \mathbb{N}=\{1,2, \ldots\}$, with the property

$$
\left\|p_{n} x\right\|_{E_{n}} \longrightarrow\|x\|_{E} \quad \text { as } n \longrightarrow \infty \text { for any } x \in E
$$

Definition 42. The sequence of elements $\left\{x_{n}\right\}, x_{n} \in E_{n}, n \in$ $\mathbb{N}$, is said to be $\mathscr{P}$-convergent to $x \in E$ if and only if $\| x_{n}-$ $p_{n} x \|_{E_{n}} \rightarrow 0$ as $n \rightarrow \infty$ and one writes this $x_{n} \stackrel{\mathscr{P}}{\rightarrow} x$.

Definition 43. The sequence of bounded linear operators $B_{n} \epsilon$ $B\left(E_{n}\right), n \in \mathbb{N}$, is said to be $\mathscr{P} \mathscr{P}$-convergent to the bounded linear operator $B \in B(E)$ if, for every $x \in E$ and for every sequence $\left\{x_{n}\right\}, x_{n} \in E_{n}, n \in \mathbb{N}$, such that $x_{n} \stackrel{\mathscr{P}}{\longrightarrow} x$, one has $B_{n} x_{n} \stackrel{\mathscr{P}}{\longrightarrow} B x$. One writes then $B_{n} \stackrel{\mathscr{P} \mathscr{P}}{\longrightarrow} B$.

For general examples of notions of $\mathscr{P}$-convergence, see [46].

Remark 44. If we put $E_{n}=E$ and $p_{n}=I$ for each $n \in \mathbb{N}$, where $I$ is the identity operator on $E$, then Definition 42 leads to the traditional pointwise convergent bounded linear operators which we denote by $B_{n} \rightarrow B$.

In the case of unbounded operators, and we know that in general infinitesimal generators are unbounded, one has to consider the notion of compatibility.

Definition 45. The sequence of closed linear operators $\left\{A_{n}\right\}$, $A_{n} \in \mathscr{C}\left(E_{n}\right), n \in \mathbb{N}$, is said to be compatible with a closed linear operator $A \in \mathscr{C}(E)$ if and only if, for each $x \in D(A)$, there is a sequence $\left\{x_{n}\right\}, x_{n} \in D\left(A_{n}\right) \subseteq E_{n}, n \in \mathbb{N}$, such that $x_{n} \stackrel{\mathscr{P}}{\rightarrow} x$ and $A_{n} x_{n} \stackrel{\mathscr{P}}{\longrightarrow} A x$. One writes $\left(A_{n}, A\right)$ are compatible.

Usually in practice, Banach spaces $E_{n}$ are finite dimensional, although, in general, say for the case of a closed operator $A$, we have $\operatorname{dim} E_{n} \rightarrow \infty$ and $\left\|A_{n}\right\|_{B\left(E_{n}\right)} \rightarrow \infty$ as $n \rightarrow \infty$.

Theorem 46 (see [48]). Let the operators $A$ and $A_{n}$ generate analytic $C_{0}$-semigroups. The following conditions $(A)$ and $\left(B_{1}\right)$ are equivalent to condition $\left(C_{1}\right)$.

(A) Consistency: there exists $\lambda \in \rho(A) \cap \cap_{n} \rho\left(A_{n}\right)$ such that the resolvents converge $\left(\lambda I_{n}-A_{n}\right)^{-1} \stackrel{\mathscr{P} \mathscr{P}}{\longrightarrow}(\lambda I-A)^{-1}$.

$\left(B_{1}\right)$ Stability: there are some constants $M_{2} \geq 1$ and $\omega_{2}$ independent of $n$ such that, for any $\operatorname{Re} \lambda>\omega_{2}$,

$$
\left\|\left(\lambda I_{n}-A_{n}\right)^{-1}\right\| \leq \frac{M_{2}}{\left|\lambda-\omega_{2}\right|} \quad \forall n \in \mathbb{N} .
$$


$\left(C_{1}\right)$ Convergence: for any finite $\mu>0$ and some $0<\theta<$ $\pi / 2$, one has

$$
\max _{\eta \in \Sigma(\theta, \mu)}\left\|\exp \left(\eta A_{n}\right) u_{n}^{0}-p_{n} \exp (\eta A) u^{0}\right\| \longrightarrow 0
$$

as $n \rightarrow \infty$ whenever $u_{n}^{0} \stackrel{\mathscr{P}}{\rightarrow} u^{0}$. Here $\Sigma(\theta, \mu)=\{z \in \Sigma(\theta)$ : $|z| \leq \mu\}$ and $\Sigma(\theta)=\{z \in \mathbb{C}:|\arg z| \leq \theta\}$.

For $C_{0}$-cosine operator functions, the following $\mathrm{ABC}$ Theorem holds.

Theorem 47 (see [6]). Let the operators $A$ and $A_{n}$ generate $C_{0}$-cosine operator functions. The following conditions $(A)$ and $\left(B^{\prime}\right)$ are equivalent to condition $\left(C^{\prime}\right)$.

(A) Compatability: there exists $\lambda \in \rho(A) \cap \cap_{n} \rho\left(A_{n}\right)$ such that the resolvents converge $\left(\lambda I_{n}-A_{n}\right)^{-1} \stackrel{\mathscr{P} \mathscr{P}}{\longrightarrow}(\lambda I-$ $A)^{-1}$.

$\left(B^{\prime}\right)$ Stability: there are some constants $M \geq 1$ and $\omega \geq 0$ such that

$$
\left\|C\left(t, A_{n}\right)\right\| \leq M e^{\omega t}, \quad t \geq 0, n \in \mathbb{N} .
$$

$\left(C^{\prime}\right)$ Convergence: for any finite $T>0$, one has

$$
\max _{t \in[0, T]}\left\|C\left(t, A_{n}\right) u_{n}^{0}-p_{n} C(t, A) u^{0}\right\| \longrightarrow 0
$$

as $n \rightarrow \infty$, whenever $u_{n}^{0} \stackrel{\mathscr{P}}{\longrightarrow} u^{0}$.

\section{Discrete WMR Inequalities}

One can also consider the problem of obtaining maximal regularity for difference schemes for second order equations in case of $C_{\tau_{n}}\left([0, T] ; E_{n}^{\theta}\right), C_{\tau_{n}}^{\alpha}\left([0, T] ; E_{n}\right)$, and $L_{\tau_{n}}^{p}\left([0, T] ; E_{q, n}^{\theta}\right)$ spaces, where $E_{q, n}^{\theta}, E_{n}^{\alpha}$ are interpolation spaces.

6.1. Discrete Maximal Regularity for the First Order Equations. The following Cauchy problems in Banach spaces $E_{n}$ are the semidiscrete approximation of (1):

$$
\begin{gathered}
u_{n}^{\prime}(t)=A_{n} u_{n}(t)+f_{n}(t), \quad t \in[0, T] ; \\
u_{n}(0)=u_{n}^{0},
\end{gathered}
$$

where the operators $A_{n}$ generate $C_{0}$-semigroups, $A_{n}$ and $A$ are compatible, and $u_{n}^{0} \stackrel{\mathscr{P}}{\rightarrow} u^{0}$ and $f_{n}(\cdot) \stackrel{\mathscr{P}}{\longrightarrow} f(\cdot)$ in appropriate sense. We assume that conditions $(A)$ and $\left(B_{1}\right)$ from Theorem $\mathrm{ABC}$ for $C_{0}$-semigroups are satisfied.

Here we are going to describe the discretization of (75) in time. The simplest difference scheme (Rothe scheme) is

$$
\begin{gathered}
\frac{U_{n}^{k}-U_{n}^{k-1}}{\tau_{n}}=A_{n} U_{n}^{k}+\varphi_{n}^{k}, \quad k \in\left\{1, \ldots,\left[\frac{T}{\tau_{n}}\right]\right\}, \\
\bar{U}_{n}^{0}=u_{n}^{0},
\end{gathered}
$$

where, for example, in the case of $f_{n}(\cdot) \in C\left([0, T] ; E_{n}\right)$, one can set $\varphi_{n}^{k}=f_{n}\left(k \tau_{n}\right), k \in\{1, \ldots, K\}, K=\left[T / \tau_{n}\right]$, and, in the case $f_{n} \in L^{1}\left([0, T] ; E_{n}\right)$, one can set

$$
\varphi_{n}^{k}=\frac{1}{\tau_{n}} \int_{t_{k-1}}^{t_{k}} f_{n}(s) d s, \quad t_{k}=k \tau_{n}, k \in\{1, \ldots, K\} .
$$

Theorem 48 (see [1]). Let condition $\left(B_{1}\right)$ be satisfied. Problem (76) is stable in the space $C_{\tau_{n}}\left([0, T] ; E_{n}\right)$; that is,

$$
\left\|\bar{U}_{n}\right\|_{C_{\tau_{n}}\left([0, T] ; E_{n}\right)} \leq C\left(\left\|\bar{\varphi}_{n}\right\|_{C_{\tau_{n}}\left([0, T] ; E_{n}\right)}+\left\|u_{n}^{0}\right\|\right) .
$$

Theorem 49 (see [1]). Let condition $\left(B_{1}\right)$ be satisfied. Problem (76) is almost coercively stable in the space $C_{\tau_{n}}\left([0, T] ; E_{n}\right)$; that is,

$$
\begin{gathered}
\left\|A_{n} \bar{U}_{n}\right\|_{C_{\tau_{n}}\left([0, T] ; E_{n}\right)} \\
\leq M\left(\left\|A_{n} u_{n}^{0}\right\|_{E_{n}}+\min \left(\ln \left(\frac{1}{\tau_{n}}\right), 1+\left|\ln \left\|A_{n}\right\|\right|\right)\right. \\
\left.\cdot\left\|\bar{\varphi}_{n}\right\|_{C_{\tau_{n}}\left([0, T] ; E_{n}\right)}\right) .
\end{gathered}
$$

Theorem 50 (see [49]). Let condition $\left(B_{1}\right)$ be satisfied. Problem (76) is coercively stable in the space $C_{\tau_{n}}\left([0, T] ; E_{n}^{\theta}\right)$; that is,

$$
\left\|A_{n} \bar{U}_{n}\right\|_{C_{\tau_{n}}\left([0, T] ; E_{n}^{\theta}\right)} \leq M\left(\left\|A_{n} u_{n}^{0}\right\|_{E_{n}^{\theta}}+\left\|\bar{\varphi}_{n}\right\|_{C_{\tau_{n}}\left([0, T] ; E_{n}^{\theta}\right)}\right) .
$$

Denote by $C_{\tau_{n}}^{\alpha, 0}\left([0, T] ; E_{n}\right), 0<\alpha<1$, the space of the elements $\bar{\varphi}_{n}$ with the norm

$$
\begin{aligned}
\left\|\bar{\varphi}_{n}\right\|_{C_{\tau_{n}}^{\alpha, 0}\left([0, T] ; E_{n}\right)}= & \max _{0 \leq k \leq K}\left\|\varphi_{n}^{k}\right\|_{E_{n}} \\
& +\max _{1 \leq k<k+l \leq K}\left\|\varphi_{n}^{k+l}-\varphi_{n}^{k}\right\|_{E_{n}}\left(\tau_{n} k\right)^{\alpha}\left(l \tau_{n}\right)^{-\alpha} .
\end{aligned}
$$

Theorem 51 (see [50]). Let condition $\left(B_{1}\right)$ hold. Then scheme (76) is coercively well-posed in $C_{\tau_{n}}^{\alpha, 0}\left([0, T] ; E_{n}\right)$ with $0<\alpha<1$; that is,

$$
\begin{aligned}
& \left\|A_{n} \bar{U}_{n}\right\|_{C_{\tau_{n}}^{\alpha, 0}\left([0, T] ; E_{n}\right)} \\
& \quad \leq \frac{M}{\alpha(1-\alpha)}\left(\left\|A_{n} u_{n}^{0}\right\|_{E_{n}}+\left\|\bar{\varphi}_{n}\right\|_{C_{\tau_{n}}^{\alpha, 0}\left([0, T] ; E_{n}\right)}\right) .
\end{aligned}
$$

Roughly speaking, assumption $\left(B_{1}\right)$ is necessary and sufficient for the coercive well-posedness in $C_{\tau_{n}}^{\alpha, 0}\left([0, T] ; E_{n}\right)$ space.

Denote by $L_{\tau_{n}}^{p}\left([0, T] ; E_{n}\right), 1 \leq p<\infty$, the space of elements $\bar{\varphi}_{n}$ with the norm

$$
\left\|\bar{\varphi}_{n}\right\|_{L_{\tau_{n}\left([0, T] ; E_{n}\right)}^{p}}=\left(\sum_{j=0}^{K}\left\|\varphi_{n}^{k}\right\|_{E_{n}}^{p} \tau_{n}\right)^{1 / p} .
$$


Theorem 52 (see [50]). Let condition $\left(B_{1}\right)$ hold. Let difference scheme (76) be coercively well-posed in $L_{\tau_{n}}^{p_{0}}\left([0, T] ; E_{n}\right)$ for some $1<p_{0}<\infty$. Then it is coercively well-posed in $L_{\tau_{n}}^{p}\left([0, T] ; E_{n}\right)$ for any $1<p<\infty$ and

$$
\begin{aligned}
& \left\|A_{n} \bar{U}_{n}\right\|_{L_{\tau_{n}}^{p}\left([0, T] ; E_{n}\right)}+\max _{0 \leq k \leq K}\left\|\bar{U}_{n}^{k}\right\|_{E_{n, 1-1 / p}} \\
& \leq \frac{M p^{2}}{p-1}\left(\left\|\bar{\varphi}_{n}\right\|_{L_{\tau_{n}}^{p}\left([0, T] ; E_{n}\right)}+\left\|\bar{U}_{n}^{0}\right\|_{1-1 / p}\right) .
\end{aligned}
$$

It should be noted that, in contrast to the case of $C^{\alpha, 0}$ space, the analyticity of the $\operatorname{semigroup} \exp (\cdot A)$ is not enough for the coercive well-posedness in $L^{p}([0, T] ; E)$ space $[51]$; therefore, to state coercive well-posedness in $L^{p}([0, T] ; E)$, we need some additional assumptions.

Theorem 53 (see [50]). Let $1<p, q<\infty, 0<\alpha<1$, and let condition $\left(B_{1}\right)$ hold. Then the difference scheme (76) is coercively well-posed in $L_{\tau_{n}}^{p}\left([0, T] ; E_{n, \alpha, q}\right)$; that is,

$$
\begin{aligned}
& \left\|A_{n} \bar{U}_{n}\right\|_{L_{\tau_{n}}^{p}\left([0, T] ; E_{n, \alpha, q}\right)}+\max _{0 \leq k \leq K}\left\|U_{n}^{k}\right\|_{E_{n, 1-1 / p}} \\
& \leq \frac{M p^{2}}{(p-1) \alpha(1-\alpha)}\left(\left\|\bar{\varphi}_{n}\right\|_{L_{\tau_{n}}^{p}\left([0, T] ; E_{n, \alpha, q}\right)}+\left\|U_{n}^{0}\right\|_{1-1 / p}\right),
\end{aligned}
$$

where $E_{n, \alpha, q}$ is the interpolation space $\left(E_{n}, D\left(A_{n}\right)\right)_{\alpha, q}$ with the norm

$$
\left\|u_{n}\right\|_{E_{n, \alpha, q}}=\left(\int_{0}^{\infty}\left\|\lambda^{\alpha} A_{n}\left(\lambda-A_{n}\right)^{-1}\right\|_{E_{n}}^{q} \frac{d \lambda}{\lambda}\right)^{1 / q} .
$$

For the general Banach space $E_{n}$, we have the following results. Assume that $A_{n}$ are generators of the analytic semigroups $\exp \left(t A_{n}\right), t \in \mathbb{R}_{+}$, of linear bounded operators such that stability condition $\left(B_{1}\right)$ holds with $\omega_{2} \leq 0$.

Theorem 54 (see [52]). Let condition $\left(B_{1}\right)$ hold. Then the solution of difference scheme (76) is almost coercively stable; that is,

$$
\begin{aligned}
& \left\|A_{n} \bar{U}_{n}\right\|_{L_{\tau_{n}}^{p}\left([0, T] ; E_{n}\right)} \\
& \leq M\left(\left\|U_{n}^{0}\right\|_{1-1 / p}+\min \left\{\log \frac{1}{\tau_{n}}, 1+\left|\log \left\|A_{n}\right\|_{B\left(E_{n}\right)}\right|\right\}\right. \\
& \left.\cdot\left\|\bar{\varphi}_{n}\right\|_{L_{\tau_{n}}^{p}\left([0, T] ; E_{n}\right)}\right)
\end{aligned}
$$

holds for any $p \geq 1$, where $M$ does not depend on $\tau_{n}, u_{n}^{0}$, or $\varphi_{n}$.

Theorem 55 (see [52]). Let condition $\left(B_{1}\right)$ hold and let $E_{n}$ be UMD Banach spaces uniformly in $n$ in the sense of boundedness of the norms of Hilbert transforms. Assume also that the set $\left\{\lambda\left(\lambda I_{n}-A_{n}\right)^{-1}: \lambda \in i \mathbb{R}, \lambda \neq 0\right\}$ is $R$-bounded with the $R$ boundedness constant independent of $n$. Then the solution of difference scheme (76) is coercively stable; that is,

$$
\left\|A_{n} \bar{U}_{n}\right\|_{L_{\tau_{n}}^{p}\left(\mathbb{Z}_{+} ; E_{n}\right)} \leq M\left\|\bar{\varphi}_{n}\right\|_{L_{\tau_{n}}^{p}\left(\mathbb{Z}_{+} ; E_{n}\right)}
$$

holds for any $p \geq 1$, where $M$ does not depend on $\tau_{n}, u_{n}^{0}$, or $\bar{\varphi}_{n}$.
The interpretation of discrete coercive inequality and discrete semigroup defines the convolution operator in the form $\breve{A}_{n} \sum_{j=0}^{k} T_{n}^{k-j} Q_{n} \varphi_{n} \tau_{n}$ with some bounded operator $Q_{n} \epsilon$ $B\left(E_{n}\right)$, which usually has smoothness property. Boundedness of the convolution operator in $L_{\tau_{n}}^{p}\left(\mathbb{Z}_{+} ; E_{n}\right)$ space implies discrete coercive well-posedness in $L_{\tau_{n}}^{p}\left(\mathbb{Z}_{+} ; E_{n}\right)$.

Definition 56. The discrete semigroup $T_{n}(\cdot)$ with generators $\breve{A}_{n}$ is said to generate coercive well-posedness on $L_{\tau_{n}}^{p}\left(\mathbb{Z}_{+} ; E_{n}\right)$ spaces if the corresponding convolution operators $\varphi_{n} \mapsto$ $\left\{\breve{A}_{n} \sum_{j=0}^{k} T_{n}^{k-j} Q_{n} \varphi_{n}^{j} \tau_{n}\right\}$ are continuous on $L_{\tau_{n}}^{p}\left(\mathbb{Z}_{+} ; E_{n}\right)$ spaces and such convolution operators are bounded with a constant which does not depend on $n$.

Concerning the previous definition, let us stress our main assumption on Hilbert transform on general approximation scheme. Up to the end of this section we will assume that the Hilbert transforms $H f(t)=(1 / \pi) P V-\int_{-\infty}^{\infty}(1 /(t-s)) f_{n}(s) d s$ extend to bounded operators on $L^{p}\left(\mathbb{R} ; E_{n}\right)$ for some (all) $p \in$ $(1, \infty)$, such that all of them are bounded by a constant which does not depend on $n$. This assumption holds if all $E_{n}$ can be embedded into a fixed space $L^{p}(\Omega)$ with $1<p<\infty$.

Theorem 57 (see [52]). Let $E_{n}$ be UMD Banach spaces. Assume also that the set $\left\{\lambda\left(\lambda I_{n}-A_{n}\right)^{-1}: \lambda \in i \mathbb{R}, \lambda \neq 0\right\}$ is $R$-bounded with the R-boundedness constant which does not depend on $n$. Then the solution of Crank-Nicolson difference scheme is coercively stable; that is,

$$
\left\|\left\{\breve{A}_{n} \frac{U_{n}^{k}+U_{n}^{k-1}}{2}\right\}\right\|_{L_{\tau_{n}}^{p}\left([0, T] ; E_{n}\right)} \leq M\left\|\varphi_{n}\right\|_{L_{\tau_{n}}^{p}\left([0, T] ; E_{n}\right)}
$$

holds for any $p \geq 1$, where $M$ does not depend on $\tau_{n}, u_{n}^{0}$, or $\varphi_{n}$.

6.2. Discrete Weak Maximal Regularity for Second Order Equations. The following Cauchy problems in Banach spaces $E_{n}$ are the semidiscrete approximation of (7):

$$
\begin{gathered}
u_{n}^{\prime \prime}(t)=A_{n} u_{n}(t)+f_{n}(t), \quad t \in[0, T] ; \\
u_{n}(0)=u_{n}^{0}, \quad u_{n}^{\prime}(0)=u_{n}^{1},
\end{gathered}
$$

where the operators $A_{n}$ generate $C_{0}$-cosine operator functions, $A_{n}$ and $A$ are compatible, and $u_{n}^{0} \stackrel{\mathscr{P}}{\rightarrow} u^{0}, u_{n}^{1} \stackrel{\mathscr{P}}{\rightarrow} u^{1}$, and $f_{n}(\cdot) \stackrel{\mathscr{P}}{\rightarrow} f(\cdot)$ in appropriate sense. We assume that natural conditions $(A)$ and $\left(B^{\prime}\right)$ from Theorem 47 for $C_{0^{-}}$ cosine operator functions are satisfied.

Since we do not have in general strong maximal regularity for second order equations, we can not expect to get strong maximal regularity in the discrete case too. 
Now we are going to describe the discretization of (7) in time variable. The simplest difference scheme is

$$
\begin{array}{r}
\frac{U_{n}^{k+1}-2 U_{n}^{k}+U_{n}^{k-1}}{\tau_{n}^{2}}=A_{n} U_{n}^{k}+\varphi_{n}^{k}, \\
k \in\left\{1, \ldots,\left[\frac{T}{\tau_{n}}\right]\right\}, \\
U_{n}^{0}=u_{n}^{0}, \quad U_{n}^{1}=u_{n}\left(\tau_{n}\right),
\end{array}
$$

where, for example, in the case of $f_{n}(\cdot) \in C\left([0, T] ; E_{n}\right)$, one can set $\varphi_{n}^{k}=f_{n}\left(k \tau_{n}\right), k \in\{1, \ldots, K\}, K=\left[T / \tau_{n}\right]$, and, in the case $f_{n}(\cdot) \in L^{1}\left([0, T] ; E_{n}\right)$, one can set

$$
\varphi_{n}^{k}=\frac{1}{\tau_{n}} \int_{t_{k-1}}^{t_{k}} f_{n}(s) d s, \quad t_{k}=k \tau_{n}, k \in\{1, \ldots, K\} .
$$

Definition 58 (see [53]). The operators $A_{n}$ of $C_{0}$-cosine operator-valued function $C\left(\cdot, A_{n}\right)$ satisfy discrete KreinFattorini Condition if the following conditions hold:

(i) There exist $\mathfrak{B}_{n} \in \mathscr{C}\left(E_{n}\right)$ such that $\mathfrak{B}_{n}^{2}=A_{n}$, and $\mathfrak{B}_{n}$ commutes with any operator from $B\left(E_{n}\right)$ commuting with $A_{n}$.

(ii) The operators $\mathfrak{B}_{n}$ generate $C_{0}$-groups such that $\left\|\exp \left( \pm t \mathfrak{B}_{n}\right)\right\| \leq M_{0} e^{\omega_{0}|t|}, t \in \mathbb{R}$.

(iii) The operators $-A_{n}$ are strongly positive; that is,

$$
\left\|\left(\lambda I_{n}-A_{n}\right)^{-1}\right\| \leq \frac{M}{1+|\lambda|}, \quad \operatorname{Re} \lambda \geq 0,
$$

and $\left\|\mathfrak{B}_{n}^{-1}\right\| \leq C$ as $n \in \mathbb{N}$.

Let us denote by $\mathbb{Z}$ the set of all integer numbers.

Definition 59. The function $\mathscr{K}(m): \mathbb{Z} \rightarrow B(E)$ is called discrete cosine operator function if

$$
\begin{gathered}
\mathscr{K}(k+m)+\mathscr{K}(k-m)=2 \mathscr{K}(k) \mathscr{K}(m), \\
k, m \in \mathbb{Z}, \\
\mathscr{K}(0)=I .
\end{gathered}
$$

The generator $A_{\tau}$ of a discrete cosine operator function is associated with it by the formula $A_{\tau}=\left(2 / \tau^{2}\right)(\mathscr{K}(1)-I)$. The operator $\mathscr{K}(1)$ is called leading operator of the discrete cosine operator function.

Proposition 60 (see [54]). Assume that $\mathscr{K}(1) \in B(E)$ is any bounded linear operator. Then the discrete operator function given by relation

$$
\begin{array}{r}
\mathscr{K}(m+1)=2 \mathscr{K}(m) \mathscr{K}(1)-\mathscr{K}(m-1), \\
m \in \mathbb{Z}, \\
\mathscr{K}(0)=I
\end{array}
$$

is a discrete cosine operator function.
We consider the discrete cosine operator function in the spaces $E_{n}$ and we write $\mathscr{C}_{n}\left(t, A_{\tau_{n}, n}\right)$ for the discrete cosine family with associated generator $A_{\tau_{n}, n}$, where $t=k \tau_{n}, k \in \mathbb{Z}$, and $\tau_{n}>0$ is the step discretization in time. So in this way the discrete cosine operator function is a function of argument $t=k \tau_{n}$ with leading operator $\mathscr{C}_{n}\left(\tau_{n}, A_{\tau_{n}, n}\right) \in B\left(E_{n}\right)$. The choice of the leading operator could be different in the sense that $\mathscr{C}_{n}\left(\tau_{n}, A_{\tau_{n}, n}\right)=I_{n}+\left(\tau_{n}^{2} / 2\right) A_{\tau_{n}, n}$ with different choice of $A_{\tau_{n}, n}$. One can take $A_{\tau_{n}, n}=A_{n}$, say from (90). Sometimes they use a different choice of $A_{\tau_{n}, n}$ (see the paragraph before the formula (103)).

Remark 61. As was mentioned in Proposition 11, the $C_{0}$ cosine operator function in general can not be represented in the form of $C_{0}$-semigroups generated by $\mathfrak{B}$. In the meantime, discrete cosine operator function can anytime be represented in such form as a power of bounded operators $[55,56]$.

Definition 62. The function $\delta(k): \mathbb{Z} \rightarrow B(E)$ is called discrete sine operator function associated with discrete cosine operator function $\mathscr{K}(m)$ if

$$
\begin{gathered}
\mathcal{S}(k+m)+\mathcal{S}(k-m)=2 \mathcal{S}(k) \mathscr{K}(m), \\
k, m \in \mathbb{Z}, \\
\mathcal{S}(0)=0 .
\end{gathered}
$$

The operator $\mathcal{S}(1) \in B(E)$ is called leading operator of the discrete sine operator function.

Proposition 63 (see [54]). Assume that $\mathcal{S}(1) \in B(E)$ is any bounded linear operator. Then the discrete operator function given by the relation

$$
\begin{gathered}
\mathcal{S}(m+1)=2 \mathcal{S}(m) \mathscr{K}(1)-\mathcal{S}(m-1), \\
m \in \mathbb{Z}, \\
\mathcal{S}(0)=0
\end{gathered}
$$

is a discrete sine operator function.

One considers the discrete sine operator function in the spaces $E_{n}$ and writes $\mathcal{S}_{n}\left(t, A_{n}\right)$ for the discrete sine family associated with $\mathscr{C}_{n}\left(t, A_{n}\right)$, where $t=k \tau_{n}, k \in \mathbb{Z}$, and $\tau_{n}>0$ is the discretisation step in time.

Proposition 64. Assume that

$$
\begin{gathered}
\mathcal{S}_{n}\left(t, A_{\tau_{n}, n}\right)=\frac{\tau_{n}}{2} I_{n}+\tau_{n} \sum_{j=1}^{t / \tau_{n}} \mathscr{C}_{n}\left(j \tau_{n}, A_{\tau_{n}, n}\right), \\
t=m \tau_{n}, \quad m \in \mathbb{Z}, \\
\mathcal{S}_{n}\left(0, A_{\tau_{n}, n}\right)=0 .
\end{gathered}
$$

Then $\left\{\mathcal{S}_{n}\left(m \tau_{n}, A_{\tau_{n}, n}\right)\right\}_{m=1}^{\infty}$ is a discrete sine operator function. 
Proof. It is enough to check (97). So one has

$$
\begin{aligned}
\tau_{n} I_{n} & +\tau_{n} \sum_{j=1}^{m-1} \mathscr{C}_{n}\left(j \tau_{n}, A_{\tau_{n}, n}\right)+\sum_{j=1}^{m+1} \mathscr{C}_{n}\left(j \tau_{n}, A_{\tau_{n}, n}\right) \\
= & \tau_{n} I_{n}+\tau_{n} \mathscr{C}_{n}\left(\tau_{n}, A_{\tau_{n}, n}\right)+\tau_{n} \mathscr{C}_{n}\left(2 \tau_{n}, A_{\tau_{n}, n}\right) \\
& +2 \tau_{n} \sum_{j=2}^{m} \mathscr{C}_{n}\left(j \tau_{n}, A_{\tau_{n}, n}\right) \mathscr{C}_{n}\left(t, A_{\tau_{n}, n}\right) \\
= & 2\left(\frac{\tau_{n}}{2}+\tau_{n} \sum_{j=1}^{m} \mathscr{C}_{n}\left(j \tau_{n}, A_{\tau_{n}, n}\right)\right) \mathscr{C}_{n}\left(t, A_{\tau_{n}, n}\right) .
\end{aligned}
$$

Sometimes one considers the corrected sine operator function

$$
\widetilde{\mathcal{S}}_{n}\left(m \tau_{n}, A_{n}\right)=\mathcal{S}_{n}\left(m \tau_{n}, A_{n}\right)-\frac{\tau}{2} \mathscr{C}_{n}\left(m \tau_{n}, A_{n}\right), \quad m \in \mathbb{Z} .
$$

In such case, the solution of (91) is given by the formula

$$
U_{n}(t)=\mathscr{C}_{n}\left(t, A_{\tau_{n}, n}\right) U_{n}^{0}+\widetilde{\mathcal{S}}_{n}\left(t, A_{\tau_{n}, n}\right) u_{n}^{1, \tau_{n}}
$$

where $u_{n}^{1, \tau_{n}}$ satisfy the relations

$$
\left(I_{n}+\frac{\tau_{n}^{2}}{4} A_{n}\right) u_{n}^{1, \tau_{n}}=\frac{U_{n}^{1}-U_{n}^{0}}{\tau_{n}}-\frac{\tau_{n}}{2} A_{n} U_{n}^{0} .
$$

One can have a look at the following choice of discrete cosine if the leading operator of the discrete cosine operator function is taken as $\mathscr{C}_{n}\left(\tau_{n}, A_{n}\left(I_{n}-\left(\tau_{n}^{2} / 2\right) A_{n}\right)^{-1}\right)=I_{n}+$ $\left(\tau_{n}^{2} / 2\right) A_{n}\left(I_{n}-\left(\tau_{n}^{2} / 2\right) A_{n}\right)^{-1}$. In [11], it was shown that schemes (91) are stable in case of $\mathscr{C}_{n}\left(\tau_{n}, A_{n}\left(I_{n}-\left(\tau_{n}^{2} / 2\right) A_{n}\right)^{-1}\right)$, that is, when, instead of $A_{n}$ in (91), one puts the operator $A_{n}\left(I_{n}-\left(\tau_{n}^{2} / 2\right) A_{n}\right)^{-1}$. Moreover, they have shown that for a nonhomogeneous equation the following estimates hold:

$$
\begin{gathered}
\left\|U_{n}\left(k \tau_{n}\right)\right\| \leq \operatorname{M\rho }\left(\tau_{n}\right)^{k} \\
\cdot\left(\left\|\frac{U_{n}^{0}+U_{n}^{1}}{2}\right\|_{D\left(A_{n}^{\epsilon}\right)}+\left\|\frac{U_{n}^{0}-U_{n}^{1}}{\tau_{n}}\right\|_{D\left(A_{n}^{\epsilon}\right)}\right. \\
\left.+\tau_{n} \sum_{j=0}^{k-1}\left\|\varphi_{n}\left(j \tau_{n}\right)\right\|_{D\left(A_{n}^{\epsilon}\right)}\right)
\end{gathered}
$$

with $k \in \mathbb{N}, \rho\left(\tau_{n}\right)=\left(1+c_{0} \tau_{n} / \sqrt{2}+c_{0}^{2} \tau_{n}^{2} / 4\right)^{1 / 2}$, and any small $\epsilon>0$.

Theorem 65 (see [9]). Let the operators $A$ and $A_{n}$ generate $C_{0}$-cosine operator functions and discrete cosine operator function, respectively. The following conditions $(A)$ and $\left(B^{\prime \prime}\right)$ are equivalent to condition $\left(C^{\prime \prime}\right)$.

(A) Compatability: there exists $\lambda \in \rho(A) \cap \cap_{n} \rho\left(A_{n}\right)$ such that the resolvents converge $\left(\lambda I_{n}-A_{n}\right)^{-1} \rightarrow(\lambda I-A)^{-1}$.

$\left(B^{\prime \prime}\right)$ Stability: there are some constants $M_{1} \geq 1$ and $\omega_{1} \geq 0$ such that

$$
\left\|\mathscr{C}_{n}\left(t, A_{n}\right)\right\| \leq M_{1} e^{\omega_{1} t}, \quad t \geq 0, n \in \mathbb{N} .
$$

$\left(C^{\prime \prime}\right)$ Convergence: for any finite $T>0$ one has

$$
\max _{t \in[0, T]}\left\|\mathscr{C}_{n}\left(t, A_{n}\right) u_{n}^{0}-p_{n} C(t, A) u^{0}\right\| \longrightarrow 0
$$

as $n \rightarrow \infty$, whenever $u_{n}^{0} \stackrel{\mathscr{P}}{\rightarrow} u^{0}$

Theorem 66. Assume that condition $\left(B^{\prime \prime}\right)$ holds. Then the scheme

$$
\begin{aligned}
& \frac{U_{n}^{k+1}-2 U_{n}^{k}+U_{n}^{k-1}}{\tau_{n}^{2}} \\
& =A_{n}\left(I_{n}-\frac{\tau_{n}^{2}}{2} A_{n}\right)^{-1} U_{n}^{k}+\varphi_{n}^{k}, \\
& k \in\left\{1, \ldots,\left[\frac{T}{\tau_{n}}\right]\right\}, \\
& U_{n}^{0}=u_{n}^{0}, \quad U_{n}^{1}=u_{n}\left(\tau_{n}\right),
\end{aligned}
$$

that is, scheme (91) with operator $A_{n}$ replaced by $A_{n}\left(I_{n}-\left(\tau_{n}^{2} /\right.\right.$ 2) $\left.A_{n}\right)^{-1}$, is almost weakly coercively stable in $C_{n}\left([0, T] ; E_{n}^{\theta}\right)$ in the following sense:

$$
\begin{gathered}
\left\|A_{n}\left(I_{n}-\frac{\tau_{n}^{2}}{2} A_{n}\right)^{-1} U_{n}\left(k \tau_{n}\right)\right\|_{E_{n}^{\theta}} \\
\leq M \rho\left(\tau_{n}\right)^{k}\left(\left\|A_{n}\left(I_{n}-\frac{\tau_{n}^{2}}{2} A_{n}\right)^{-1} \frac{U_{n}^{0}+U_{n}^{1}}{2}\right\|_{D\left(A_{n}^{\theta+\epsilon}\right)}\right. \\
+\left\|A_{n}\left(I_{n}-\frac{\tau_{n}^{2}}{2} A_{n}\right)^{-1} \frac{U_{n}^{0}-U_{n}^{1}}{\tau_{n}}\right\|_{D\left(A_{n}^{\theta+\epsilon}\right)} \\
\left.+\tau_{n} \sum_{j=0}^{k-1}\left\|\varphi_{n}^{\prime}\left(j \tau_{n}\right)\right\|_{D\left(A_{n}^{\theta+\epsilon}\right)}\right)
\end{gathered}
$$

with $k \in \mathbb{N}, \rho\left(\tau_{n}\right)=\left(1+c_{0} \tau_{n} / \sqrt{2}+c_{0}^{2} \tau_{n}^{2} / 4\right)^{1 / 2}, \epsilon>0$.

Proof. We follow the proof for (28) in [11], but we get an estimate for $A_{n} U_{n}$ instead of $U_{n}$. Integration by parts gives us the discrete derivative of $\varphi_{n}(\cdot)$ which we denote by $\varphi_{n}^{\prime}\left(j \tau_{n}\right)$. The product $k \tau_{n}$ is bounded by $T$, so one can get an estimate in discrete spaces $C_{n}\left([0, T] ; E_{n}^{\theta}\right)$ for

$$
\begin{aligned}
U_{n}^{k}= & \frac{1}{2 \pi i} \int_{\Gamma} Q_{n}(z)\left(z I_{n}-A_{n}\right)^{-1} d z U_{n}^{0} \\
& +\frac{1}{2 \pi i} \int_{\Gamma} Q_{n}(z)\left(z I_{n}-A_{n}\right)^{-1} d z U_{n}^{1} \\
& +\frac{1}{2 \pi i} \int_{\Gamma} \sum_{j=0}^{k-1} R_{k-j}(z)\left(z I_{n}-A_{n}\right)^{-1} \varphi_{n}^{\prime}\left(j \tau_{n}\right) d z
\end{aligned}
$$




\section{Difference Schemes for Hyperbolic Equations}

A large cycle of works on difference schemes for hyperbolic partial differential equations (see, e.g., $[48,57-61]$ and the references given therein), in which stability was established under the assumption that the magnitude of the grid steps $\tau$ and $h$ with respect to the time and space variables are connected. In abstract terms, this means, in particular, that the condition $\tau\left\|A_{h}\right\| \rightarrow 0$ when $\tau \rightarrow 0$ is satisfied.

Of great interest is the study of absolutely stable difference schemes of a high order of accuracy for hyperbolic partial differential equations, in which stability was established without any assumptions with respect to the grid steps $\tau$ and $h$. Such type of stability inequalities for the solutions of the first order of accuracy difference scheme for the differential equations of hyperbolic type were established for the first time in [62]. The first and second order of accuracy difference schemes approximately solving the abstract initial value problem for hyperbolic equations in Hilbert spaces were presented in [63]. Applying the operator approach, the stability estimates for the solution of these difference schemes were obtained.

In this survey section, we present results on stability and convergence of absolutely stable difference schemes for hyperbolic partial differential equations. Sections 7.1 and 7.2 are devoted to a Cauchy problem for hyperbolic equations. Section 7.1 is based on results of $[2,7]$. Section 7.2 is based on results of [64-69]. In mathematical modeling, partial differential equations are used together with boundary conditions specifying the solution on the boundary of the domain. In some cases, classical boundary conditions cannot describe a process or phenomenon precisely. Therefore, mathematical models of various physical, chemical, biological, or environmental processes often involve nonclassical conditions. Such conditions are usually identified as nonlocal boundary conditions and reflect situations when the data on the domain boundary cannot be measured directly, or when the data on the boundary depend on the data inside the domain. Sections 7.3 and 7.4 are devoted to nonlocal boundary value problems for hyperbolic and mixed types of partial differential equations. Section 7.3 is based on results of [7076]. Section 7.4 is based on results of [77-91]. Section 7.5 is devoted to stochastic hyperbolic equations. It is based on results of $[92,93]$. Section 7.6 is devoted to fractional hyperbolic differential and difference equations. It is based on results of [94-97]. Finally, Section 7.7 is devoted to singular perturbation hyperbolic problems. It is based on results of [98-101].

This survey section does not touch the results of [102106] on integral inequalities with two dependent limits, on the theory of integral-differential equations of hyperbolic type, and on difference schemes for the approximate solution of these problems or of $[107,108]$ on the equations of the second order with a small parameter at the highest derivatives and on the investigation of singular hyperbolic equations. Moreover, we do not discuss results on the stability of the Goursat problem for hyperbolic equations and an initial-boundary value problem for a system of differential equations of first order with nonlocal condition and difference schemes for the approximate solution of these problems, for which the reader is referred to [109-114].

7.1. A Cauchy Problem. We consider the abstract Cauchy problem for hyperbolic equations

$$
\begin{gathered}
v^{\prime \prime}(t)+A v(t)=f(t) \quad(0 \leq t \leq T), \\
v(0)=\varphi, \quad v^{\prime}(0)=\psi
\end{gathered}
$$

in Hilbert space $H$ with the self-adjoint positive definite operator $A$. Function $v(t)$ is called a solution of problem (109) if the following conditions are satisfied:

(i) $v(t)$ is twice continuously differentiable on the segment $[0, T]$. The derivatives at the endpoints of the segment are understood as the appropriate unilateral derivatives.

(ii) The element $v(t)$ belongs to $D(A)$ for all $t \in[0, T]$ and the function $A v(t)$ is continuous on the segment $[0, T]$.

(iii) $v(t)$ satisfies the equations and initial conditions (109).

If the function $f(t)$ is not only continuous, but also continuously differentiable on $[0, T], \varphi \in D(A)$, and $\psi \in$ $D\left(A^{1 / 2}\right)$, it is easy to show that the formula

$$
v(t)=C(t, A) \varphi+S(t, A) \psi+\int_{0}^{t} S(t-\lambda, A) f(\lambda) d \lambda
$$

gives a solution of problem (109). Here [115]

$$
\begin{gathered}
C(t, A)=\frac{e^{i t A^{1 / 2}}+e^{-i t A^{1 / 2}}}{2}, \\
S(t, A)=A^{-1 / 2} \frac{e^{i t A^{1 / 2}}-e^{-i t A^{1 / 2}}}{2 i} .
\end{gathered}
$$

Theorem 67. Suppose that $\varphi \in D(A), \psi \in D\left(A^{1 / 2}\right)$, and $f(t)$ are continuously differentiable on $[0, T]$ function. Then there is a unique solution of problem (109) and the stability inequalities

$$
\begin{gathered}
\max _{0 \leq t \leq T}\|v(t)\|_{H} \\
\leq M\left[\|\varphi\|_{H}+\left\|A^{-1 / 2} \psi\right\|_{H}+\max _{0 \leq t \leq T}\left\|A^{-1 / 2} f(t)\right\|_{H}\right] \\
\max _{0 \leq t \leq T}\left\|A^{1 / 2} v(t)\right\|_{H} \\
\leq M\left[\left\|A^{1 / 2} \varphi\right\|_{H}+\|\psi\|_{H}+\max _{0 \leq t \leq T}\|f(t)\|_{H}\right] \\
\max _{0 \leq t \leq T}\left\|\frac{d^{2} v(t)}{d t^{2}}\right\|_{H}+\max _{0 \leq t \leq T}\|A v(t)\|_{H} \\
\leq M\left[\|A \varphi\|_{H}+\left\|A^{-1 / 2} \psi\right\|_{H}\right. \\
\left.+\|f(0)\|_{H}+\int_{0}^{T}\left\|f^{\prime}(t)\right\|_{H} d t\right]
\end{gathered}
$$

hold, where $M$ does not depend on $f(t), t \in[0, T]$, or $\varphi, \psi$. 
Now, we consider the application of abstract Theorem 67. First, we consider the mixed problem for the hyperbolic equation

$$
\begin{aligned}
& u_{t t}(t, x)-\left(a(x) u_{x}\right)_{x}+u(t, x)=f(t, x) \text {, } \\
& 0 \leq t \leq T, \quad 0 \leq x \leq L, \\
& u(0, x)=\varphi(x), \quad u_{t}(0, x)=\psi(x), \\
& 0 \leq x \leq L, \\
& u(t, 0)=u(t, L), \quad u_{x}(t, 0)=u_{x}(t, L), \\
& 0 \leq t \leq T .
\end{aligned}
$$

Problem (113) has a unique smooth solution $u(t, x)$ for the smooth $a(x)>0, a(0)=a(L)(x \in[0, L]), \varphi(x)$, $\psi(x)(x \in[0, L])$, and $f(t, x)(t \in[0, T], x \in[0, L])$ functions. This allows us to reduce mixed problem (113) to initial value problem (109) in the Hilbert space $H=L_{2}[0, L]$ with self-adjoint positive definite operator $A$ defined by (113). Let us give a number of corollaries of Theorem 67 .

Theorem 68. For solutions of mixed problem (113), the stability inequalities

$$
\begin{gathered}
\max _{0 \leq t \leq T}\|u(t, \cdot)\|_{W_{2}^{1}[0, L]} \\
\leq M\left[\max _{0 \leq t \leq T}\|f(t, \cdot)\|_{L_{2}[0, L]}+\|\varphi\|_{W_{2}^{1}[0, L]}+\|\psi\|_{L_{2}[0, L]}\right] \\
\max _{0 \leq t \leq T}\|u(t, \cdot)\|_{W_{2}^{2}[0, L]}+\max _{0 \leq t \leq T}\left\|u_{t t}(t, \cdot)\right\|_{L_{2}[0, L]} \\
\leq M\left[\max _{0 \leq t \leq T}\left\|f_{t}(t, \cdot)\right\|_{L_{2}[0, L]}+\|f(0, \cdot)\|_{L_{2}[0, L]}\right. \\
\left.+\|\varphi\|_{W_{2}^{2}[0, L]}+\|\psi\|_{W_{2}^{1}[0, L]}\right]
\end{gathered}
$$

hold, where $M$ does not depend on $f(t, x)$ or $\varphi(x), \psi(x)$.

The proof of Theorem 68 is based on Theorem 67 and the symmetry properties of the space operator generated by problem (113).

Second, let $\Omega \subset \mathbb{R}^{n}$ be the bounded open domain with smooth boundary $S, \bar{\Omega}=\Omega \cup S$. In $[0, T] \times \Omega$, we consider the mixed boundary value problem for hyperbolic equations

$$
\begin{gathered}
u_{t t}(t, x)-\sum_{r=1}^{n}\left(a_{r}(x) u_{x_{r}}\right)_{x_{r}}=f(t, x), \\
x=\left(x_{1}, \ldots, x_{n}\right) \in \bar{\Omega}, \quad 0 \leq t \leq T, \\
u(0, x)=\varphi(x), \quad \frac{\partial u(0, x)}{\partial t}=\psi(x), \quad x \in \bar{\Omega}, \\
u(t, x)=0, \quad x \in S, \quad 0 \leq t \leq T,
\end{gathered}
$$

where $a_{r}(x),(x \in \bar{\Omega}), \varphi(x), \psi(x)(x \in \bar{\Omega})$, and $f(t, x)(t \in$ $[0, T], x \in \bar{\Omega})$ are given smooth functions and $a_{r}(x)>0$. We introduce the Hilbert space $L_{2}(\bar{\Omega})$, the space of all integrable functions defined on $\bar{\Omega}$, equipped with the norm

$$
\|f\|_{L_{2}(\bar{\Omega})}=\left\{\int \cdots \int_{x \in \bar{\Omega}}|f(x)|^{2} d x_{1} \cdots d x_{n}\right\}^{1 / 2} .
$$

Problem (115) has a unique smooth solution $u(t, x)$ for the smooth $a_{r}(x)>0$ and $f(t, x)$ functions. This allows us to reduce mixed problem (115) to initial value problem (109) in the Hilbert space $H=L_{2}(\bar{\Omega})$ with a self-adjoint positive definite operator $A$ defined by (115).

Theorem 69. For solutions of mixed problem (115), the stability inequalities

$$
\begin{gathered}
\max _{0 \leq t \leq T}\|u(t, \cdot)\|_{W_{2}^{1}(\bar{\Omega})} \\
\leq M\left[\max _{0 \leq t \leq T}\|f(t, \cdot)\|_{L_{2}(\bar{\Omega})}+\|\varphi\|_{W_{2}^{1}(\bar{\Omega})}+\|\psi\|_{L_{2}(\bar{\Omega})}\right] \\
\max _{0 \leq t \leq T}\|u(t, \cdot)\|_{W_{2}^{2}(\bar{\Omega})}+\max _{0 \leq t \leq T}\left\|u_{t t}(t, \cdot)\right\|_{L_{2}(\bar{\Omega})} \\
\leq M\left[\max _{0 \leq t \leq T}\left\|f_{t}(t, \cdot)\right\|_{L_{2}(\bar{\Omega})}+\|f(0, \cdot)\|_{L_{2}(\bar{\Omega})}\right. \\
\left.\quad+\|\varphi\|_{W_{2}^{2}(\bar{\Omega})}+\|\psi\|_{W_{2}^{1}(\bar{\Omega})}\right]
\end{gathered}
$$

hold, where $M$ does not depend on $f(t, x)$ or $\varphi(x), \psi(x)$.

The proof of Theorem 69 is based on Theorem 67 and the symmetry properties of the space operator generated by problem (115) and the following theorem on the coercivity inequality for the solution of the elliptic differential problem in $L_{2}(\bar{\Omega})$.

Theorem 70 (see [116]). For the solution of the elliptic differential problem

$$
\begin{gathered}
-\sum_{r=1}^{n}\left(a_{r}(x) u_{x_{r}}\right)_{x_{r}}=w(x), \quad x \in \Omega, \\
u(x)=0, \quad x \in S,
\end{gathered}
$$

the coercivity inequality

$$
\sum_{r=1}^{n}\left\|u_{x_{r} x_{r}}\right\|_{L_{2}(\bar{\Omega})} \leq M\|w\|_{L_{2}(\bar{\Omega})}
$$

is valid.

Now, we consider the high order of accuracy two-step difference schemes generated by an exact difference scheme and by Taylor's decomposition on three points for the approximate solutions of initial value problem (109). On the segment $[0, T]$, we consider a uniform grid

$$
[0, T]_{\tau}=\left\{t_{k}=k \tau, k=0,1, \ldots, N, N \tau=T\right\}
$$

with step $\tau$. First, we consider the high order of accuracy two-step difference schemes generated by an exact difference scheme for the approximate solutions of initial value problem (109). 
Theorem 71 (see [2]). For the solution of initial value problem (109), one has the following exact two-step difference scheme:

$$
\begin{gathered}
\frac{1}{\tau^{2}}\left(u_{k+1}-2 u_{k}+u_{k-1}\right)=\frac{2}{\tau^{2}}(C(\tau, A)-I) u_{k}+f_{k}, \\
f_{k}=\tau^{-1}\left\{f_{1, k+1}+S(\tau, A) f_{2, k}-C(\tau, A) f_{1, k}\right\} \\
f_{1, k}=\tau^{-1} \int_{t_{k-1}}^{t_{k}} S\left(t_{k}-z, A\right) f(z) d z, \\
f_{2, k}=\tau^{-1} \int_{t_{k-1}}^{t_{k}} C\left(t_{k}-z, A\right) f(z) d z, \\
1 \leq k \leq N-1, \\
u_{0}=v(0), \\
u_{1}=C(\tau, A) v(0)+S(\tau, A) v^{\prime}(0)+\tau f_{1,1} .
\end{gathered}
$$

Let $B=A^{1 / 2}$. Suppose the operators $\left(I-e^{2 \tau i B}\right)$ and $\left(I+e^{\tau i B}\right)$ have the bounded inverses $\left(I-e^{2 \tau i B}\right)^{-1}$ and $\left(I+e^{\tau i B}\right)^{-1}$. Then this difference scheme is uniquely solvable and the following formula holds:

$$
\begin{gathered}
u_{0}=v(0), \\
u_{1}=C(\tau, A) v(0)+S(\tau, A) v^{\prime}(0)+\tau f_{1,1}, \\
u_{k}=\left[e^{-k \tau i B}-\left(I-e^{2 \tau i B}\right)^{-1}\left(e^{-k \tau i B}-e^{k \tau i B}\right)\right] u_{0} \\
+e^{\tau i B}\left(I-e^{2 \tau i B}\right)^{-1}\left(e^{-k \tau i B}-e^{k \tau i B}\right) u_{1} \\
+\tau^{2} \sum_{m=1}^{k-1} e^{\tau i B}\left(I-e^{2 \tau i B}\right)^{-1}\left(e^{-(k-m) \tau i B}-e^{(k-m) \tau i B}\right) f_{m}, \\
2 \leq k \leq N .
\end{gathered}
$$

Now, we will consider the applications of exact difference scheme (121). From (121), it is clear that for the approximate solutions of problem (109) it is necessary to approximate the expressions

$$
\begin{array}{cl}
S(\tau, A), \quad & C(\tau, A), \quad \int_{t_{k-1}}^{t_{k}} S\left(t_{k}-z, A\right) f(z) d z, \\
& \int_{t_{k-1}}^{t_{k}} C\left(t_{k}-z, A\right) f(z) d z .
\end{array}
$$

Let us remark that in constructing difference schemes it is important to know how to construct $f_{k}^{j, l}$ and $f_{1,1}^{j, l}$ such that

$$
\begin{aligned}
& \tau^{-2} \int_{t_{k}}^{t_{k+1}} S\left(t_{k+1}-z, A\right) f(z) d z-C(\tau, A) \tau^{-2} \\
& \quad \cdot \int_{t_{k-1}}^{t_{k}} S\left(t_{k}-z, A\right) f(z) d z \\
& \quad+S(\tau, A) \tau^{-2} \int_{t_{k-1}}^{t_{k}} C\left(t_{k}-z, A\right) f(z) d z \\
& \quad-f_{k}^{j, l}=o\left(\tau^{j+l}\right), \\
& \tau^{-1} \int_{0}^{\tau} S(\tau-z, A) f(z) d z-f_{1,1}^{j, l}=o\left(\tau^{j+l}\right),
\end{aligned}
$$

and the formulas of $f_{k}^{j, l}$ and $f_{1,1}^{j, l}$ are sufficiently simple. The choice of $f_{k}^{j, l}$ and $f_{1,1}^{j, l}$ is not unique. Using Taylor's formula and integration by parts, we obtain the representation

$$
\begin{aligned}
& \tau^{-2} \int_{t_{k-1}}^{t_{k}} S\left(t_{k}-z\right) f(z) d z \\
& =\sum_{m=0}^{j+l-1} \beta_{m} f^{(m)}\left(t_{k-1}\right) \\
& \quad+\tau^{-2} \int_{t_{k-1}}^{t_{k}} S\left(t_{k}-z, A\right) \\
& \cdot \int_{t_{k-1}}^{s} \frac{(s-z)^{j+l-1}}{(j+l-1) !} f^{(j+l)}(z) d z d s
\end{aligned}
$$

in which

$$
\begin{gathered}
\beta_{m}=-A^{-1} \frac{\tau^{m-2}}{m !}+A^{-1} \beta_{m-2}, \quad 2 \leq m \leq j+l-1 \\
\beta_{0}=\tau^{-2} A^{-1}(C(\tau, A)-I) \\
\beta_{1}=\tau^{-2} A^{-1}(S(\tau, A)-\tau I) .
\end{gathered}
$$

In a similar manner, one can obtain that

$$
\begin{aligned}
& \tau^{-2} \int_{t_{k-1}}^{t_{k}} C\left(t_{k}-z, A\right) f(z) d z \\
& =\sum_{m=0}^{j+l-1} \gamma_{m} f^{(m)}\left(t_{k-1}\right) \\
& \quad+\tau^{-2} \int_{t_{k-1}}^{t_{k}} C\left(t_{k}-z, A\right) \\
& \cdot \int_{t_{k-1}}^{s} \frac{(s-z)^{j+l-1}}{(j+l-1) !} f^{(j+l)}(z) d z d s,
\end{aligned}
$$


in which

$$
\begin{gathered}
\gamma_{m}=\beta_{m-1}, \quad 1 \leq m \leq j+l-1 \\
\gamma_{0}=\tau^{-2} S(\tau, A)
\end{gathered}
$$

Using the definitions of $S(\tau, A)$ and $C(\tau, A)$ and Padé fractions $R_{j, l+1}(z)$ for the function $e^{-z}$, we can write

$$
\begin{gathered}
C(\tau, A)=\frac{R_{j, l+1}(i \tau B)+R_{j, l+1}(-i \tau B)}{2}+o\left(\tau^{j+l+2}\right), \\
S(\tau, A)=B^{-1} \frac{R_{j, l+1}(i \tau B)-R_{j, l+1}(-i \tau B)}{2 i}+o\left(\tau^{j+l+2}\right) .
\end{gathered}
$$

Now, using formulas (124), (125), (127), and (129), $f_{k}^{j, l}$ and $f_{1,1}^{j, l}$ can be defined by the following formulas:

$$
\begin{aligned}
f_{k}^{j, l}= & \sum_{m=0}^{j+l-1} B_{m} f^{(m)}\left(t_{k}\right) \\
& +B^{-1} \frac{R_{j, l+1}(i \tau B)-R_{j, l+1}(-i \tau B)}{2 i} \\
& \times \sum_{m=0}^{j+l-1} \widetilde{B}_{m} f^{(m)}\left(t_{k-1}\right) \\
& -\frac{R_{j, l+1}(i \tau B)+R_{j, l+1}(-i \tau B)}{2} \sum_{m=0}^{j+l-1} B_{m} f^{(m)}\left(t_{k-1}\right), \\
& f_{1,1}^{j, l}=\sum_{m=0}^{j+l-2} \widetilde{B}_{m} f^{(m)}(0), \quad f_{0}^{j, l}=\tau f_{1,1}^{j, l},
\end{aligned}
$$

where

$$
\begin{gathered}
B_{m}=-A^{-1} \frac{\tau^{m-2}}{m !}+A^{-1} B_{m-2}, \quad 2 \leq m \leq j+l-1, \\
B_{0}=\tau^{-2} A^{-1}\left(\frac{R_{j, l+1}(i \tau B)+R_{j, l+1}(-i \tau B)}{2}-I\right), \\
B_{1}=\tau^{-2} A^{-1}\left(B^{-1} \frac{R_{j, l+1}(i \tau B)-R_{j, l+1}(-i \tau B)}{2 i}-\tau I\right), \\
\widetilde{B}_{m}=B_{m-1}, \quad 1 \leq m \leq j+l-1 ; \\
\widetilde{B}_{0}=\tau^{-2} B^{-1} \frac{R_{j, l+1}(i \tau B)-R_{j, l+1}(-i \tau B)}{2 i} .
\end{gathered}
$$

Now, using formulas (121), (130), and (131), we obtain the difference schemes $(j+l)$ th order of accuracy

$$
\begin{gathered}
\tau^{-2}\left(u_{k+1}-2 u_{k}+u_{k-1}\right)+\widetilde{A} u_{k}=f_{k}^{j, l}, \quad 1 \leq k \leq N-1, \\
u_{0}=v_{0}, \\
\widetilde{A}=2 \tau^{-2}\left(I-\frac{R_{j, l+1}(i \tau B)+R_{j, l+1}(-i \tau B)}{2}\right), \\
\tau^{-1}\left(u_{1}-u_{0}\right)=\tau^{-1}\left(\frac{R_{j, l}(i \tau B)+R_{j, l}(-i \tau B)}{2}-I\right) v_{0} \\
+\tau^{-1} B^{-1} \frac{R_{j, l}(i \tau B)-R_{j, l}(-i \tau B)}{2 i} v^{\prime}(0) \\
+f_{0}^{j, l},
\end{gathered}
$$

for the approximate solution of initial value problem (109).

Note that difference schemes (133) for $j=l, j=l-$ 1 , and $j=l+1$ include difference schemes of arbitrary high order of approximation. Moreover, the corresponding functions $\left|R_{j, l+1}(z)\right|$ tend to 0 as $z \rightarrow \infty$ for $j=l-1, l$ and $\left|R_{j, l+1}(z)\right|=1$ for $j=l+1$. Such difference schemes are the simplest, in the sense that the degrees of the denominators of the corresponding Padé approximants of the function $\exp \{-z\}$ are minimal for a fixed order of approximation of the difference schemes.

Suppose the operators $\left(I-R_{j, l}^{2}(i \tau B)\right)$ and $\left(I+R_{j, l}(i \tau B)\right)$ have the bounded inverses $\left(I-R_{j, l}^{2}(i \tau B)\right)^{-1}$ and $(I+$ $\left.R_{j, l}(i \tau B)\right)^{-1}$. It is clear that this problem is uniquely solvable and the following formula holds:

$$
\begin{aligned}
& u_{k}= {\left[R_{j, l}^{k}(-i \tau B)-\left(I-R_{j, l}^{2}(i \tau B)\right)^{-1}\right.} \\
&\left.\cdot\left(R_{j, l}^{k}(-i \tau B)-R_{j, l}^{k}(i \tau B)\right)\right] u_{0} \\
&+R_{j, l}(i \tau B)\left(I-R_{j, l}^{2}(i \tau B)\right)^{-1} \\
& \cdot\left(R_{j, l}^{k}(-i \tau B)-R_{j, l}^{k}(i \tau B)\right) u_{1} \\
&+\tau^{2} \sum_{m=1}^{k-1} R_{j, l}(i \tau B)\left(I-R_{j, l}^{2}(i \tau B)\right)^{-1} \\
& \cdot\left[R_{j, l}^{k-m}(-i \tau B)-R_{j, l}^{k-m}(i \tau B)\right] f_{m}^{j, l}, \\
& u_{1}=\frac{R_{j, l}(i \tau B)+R_{j, l}(-i \tau B)}{2} \varphi 2 \leq k \leq N, \\
&+ B^{-1} \frac{R_{j, l}(i \tau B)-R_{j, l}(-i \tau B)}{2 i} \psi+\tau f_{0}^{j, l}, \\
& u_{0}=\varphi .
\end{aligned}
$$


Theorem 72. Suppose the operators $\left(I-R_{j, l}^{2}(i \tau B)\right)$ and $(I+$ $\left.R_{j, l}(i \tau B)\right)$ have the bounded inverses $\left(I-R_{j, l}^{2}(i \tau B)\right)^{-1}$ and $(I+$ $\left.R_{j, l}(i \tau B)\right)^{-1}$. Suppose that $u_{0} \in D\left(B^{2}\right), u_{1}-u_{0} \in D(B)$. Then for the solution of two-step difference schemes (133) for $j=l$ and $j=l-1$ the following stability inequalities hold:

$$
\begin{gathered}
\max _{0 \leq k \leq N}\left\|u_{k}\right\|_{H} \leq M\left[\max _{1 \leq k \leq N-1}\left\|B^{-1} f_{k}^{j, l}\right\|_{H}\right. \\
\left.+\left\|B^{-1} \tau^{-1}\left(u_{1}-u_{0}\right)\right\|_{H}+\left\|u_{0}\right\|_{H}\right], \\
\max _{1 \leq k \leq N}\left\|\tau^{-1}\left(u_{k}-u_{k-1}\right)\right\|_{H}+\max _{1 \leq k \leq N}\left\|\tau^{-1}\left(I-e^{-\tau i B}\right) u_{k-1}\right\|_{H} \\
\leq M\left[\max _{1 \leq k \leq N-1}\left\|f_{k}^{j, l}\right\|_{H}+\left\|\tau^{-1}\left(u_{1}-u_{0}\right)\right\|_{H}+\left\|B u_{0}\right\|_{H}\right], \\
\max _{1 \leq k \leq N-1}\left\|\tau^{-2}\left(u_{k+1}-2 u_{k}+u_{k-1}\right)\right\|_{H}+\max _{1 \leq k \leq N-1}\left\|\widetilde{A} u_{k}\right\|_{H} \\
\leq M\left[\max _{2 \leq k \leq N-1}\left\|\tau^{-1}\left(f_{k}^{j, l}-f_{k-1}^{j, l}\right)\right\|_{H}+\left\|f_{1}^{j, l}\right\|_{H}\right. \\
\left.+\left\|B \tau^{-1}\left(u_{1}-u_{0}\right)\right\|_{H}+\left\|B^{2} u_{0}\right\|_{H}\right],
\end{gathered}
$$

where $M$ does not depend on $\tau, f_{k}^{j, l}, 1 \leq k \leq N-1$, or $u_{0}, u_{1}$.

Theorem 73. Suppose the operators $\left(I-R_{j, l}^{2}(i \tau B)\right)$ and $(I+$ $\left.R_{j, l}(i \tau B)\right)$ have the bounded inverses $\left(I-R_{j, l}^{2}(i \tau B)\right)^{-1}$ and $(I+$ $\left.R_{j, l}(i \tau B)\right)^{-1}$. Suppose that $u_{0} \in D\left(B^{3}\right), u_{1}-u_{0} \in D\left(B^{2}\right)$ and $f_{k}^{j, l} \in D(B), 1 \leq k \leq N-1$. Then for the solution of twostep difference schemes (133) for $j=l+1$ the following stability inequalities hold:

$$
\begin{aligned}
& \max _{0 \leq k \leq N}\left\|u_{k}\right\|_{H} \\
& \leq M\left[\max _{1 \leq k \leq N-1}\left\|f_{k}^{j, l}\right\|_{H}+\left\|\tau^{-1}\left(u_{1}-u_{0}\right)\right\|_{H}+\left\|B u_{0}\right\|_{H}\right], \\
& \max _{1 \leq k \leq N}\left\|\tau^{-1}\left(u_{k}-u_{k-1}\right)\right\|_{H}+\max _{1 \leq k \leq N}\left\|\tau^{-1}\left(I-e^{-\tau i B}\right) u_{k-1}\right\|_{H} \\
& \leq M\left[\max _{1 \leq k \leq N-1}\left\|B f_{k}^{j, l}\right\|_{H}+\left\|B \tau^{-1}\left(u_{1}-u_{0}\right)\right\|_{H}+\left\|B^{2} u_{0}\right\|_{H}\right], \\
& \max _{1 \leq k \leq N-1}\left\|\tau^{-2}\left(u_{k+1}-2 u_{k}+u_{k-1}\right)\right\|_{H}+\max _{1 \leq k \leq N-1}\left\|\widetilde{A} u_{k}\right\|_{H} \\
& \leq M\left[\max _{2 \leq k \leq N-1}\left\|\tau^{-1} B\left(f_{k}^{j, l}-f_{k-1}^{j, l}\right)\right\|_{H}+\left\|B f_{1}^{j, l}\right\| \|_{H}\right. \\
& \left.+\left\|B^{2} \tau^{-1}\left(u_{1}-u_{0}\right)\right\|_{H}+\left\|B^{3} u_{0}\right\|_{H}\right],
\end{aligned}
$$$$
\left\|\varphi^{h}\right\|_{L_{2}\left(\bar{\Omega}_{h}\right)}=\left(\sum_{x \in \bar{\Omega}_{h}}\left|\varphi^{h}(x)\right|^{2} h_{1} \cdots h_{n}\right)^{1 / 2} .
$$

To the differential operator $A^{x}$ generated by problem (115), we assign the difference operator $A_{h}^{x}$ by the formula

$$
A_{h}^{x} u_{x}^{h}=-\sum_{r=1}^{n}\left(a_{r}(x) u_{\bar{x}_{r}}^{h}\right)_{x_{r}, j_{r}}
$$

acting in the space of grid functions $u^{h}(x)$, satisfying the conditions $u^{h}(x)=0$ for all $x \in S_{h}$. It is known that $A_{h}^{x}$ is a self-adjoint positive definite operator in $L_{2}\left(\bar{\Omega}_{h}\right)$. With the help of $A_{h}^{x}$, we arrive at the initial value problem:

$$
\begin{gathered}
\frac{d^{2} v^{h}(t, x)}{d t^{2}}+A_{h}^{x} v^{h}(t, x)=f^{h}(t, x), \quad 0 \leq t \leq T, \quad x \in \bar{\Omega}_{h}, \\
v^{h}(0, x)=\varphi^{h}(x), \quad \frac{d v^{h}(0, x)}{d t}=\psi^{h}(x), \quad x \in \bar{\Omega}_{h}
\end{gathered}
$$

Now, abstract Theorems 72 and 73 are applied in the investigation of difference schemes of higher order of accuproblem (115) is carried out in two steps. In the first step let

We introduce the Banach space $L_{2}\left(\bar{\Omega}_{h}\right)$ of the grid functions $\varphi^{h}(x)=\left\{\varphi\left(h_{1} m_{1}, \ldots, h_{n} m_{n}\right)\right\}$ defined on $\bar{\Omega}_{h}$, equipped with the norm of mixed boundary value problem (115). The discretization of

\footnotetext{
where $M$ does not depend on $\tau, f_{k}^{j, l}, 1 \leq k \leq N-1$, or $u_{0}, u_{1}$.
} for an infinite system of ordinary differential equations. 
In the second step we replace problem (140) with difference schemes (133):

$$
\begin{aligned}
& \tau^{-2}\left(u_{k+1}^{h}(x)-2 u_{k}^{h}(x)+u_{k-1}^{h}(x)\right)+\widetilde{A_{h}^{x}} u_{k}^{h}(x)=f_{k}^{h}(x), \\
& t_{k}=k \tau, \quad 1 \leq k \leq N-1, x \in \bar{\Omega}_{h}, \\
& u_{0}^{h}(x)=\varphi^{h}(x) \text {, } \\
& \tau^{-1}\left(u_{1}^{h}(x)-u_{0}^{h}(x)\right) \\
& =\tau^{-1}\left(\frac{R_{j, l}\left(i \tau B_{h}^{x}\right)+R_{j, l}\left(-i \tau B_{h}^{x}\right)}{2}-I\right) \varphi^{h}(x) \\
& +\tau^{-1}\left(B_{h}^{x}\right)^{-1} \\
& \cdot \frac{R_{j, l}\left(i \tau B_{h}^{x}\right)-R_{j, l}\left(-i \tau B_{h}^{x}\right)}{2 i} \psi^{h}(x)+\tau f_{0}^{h}(x) \text {, } \\
& x \in \bar{\Omega}_{h} \\
& \widetilde{A_{h}^{x}}=2 \tau^{-2}\left(I-\frac{R_{j, l+1}\left(i \tau B_{h}^{x}\right)+R_{j, l+1}\left(-i \tau B_{h}^{x}\right)}{2}\right), \\
& \left(B_{h}^{x}\right)^{2}=A_{h}^{x} \\
& f_{k}^{h}(x) \\
& =\sum_{m=0}^{j+l-1} B_{m} f^{(m)}\left(t_{k}, x\right) \\
& +B^{-1} \frac{R_{j, l+1}\left(i \tau B_{h}^{x}\right)-R_{j, l+1}\left(-i \tau B_{h}^{x}\right)}{2 i} \\
& \times \sum_{m=0}^{j+l-1} \widetilde{B}_{m} f^{(m)}\left(t_{k-1}, x\right) \\
& -\frac{R_{j, l+1}\left(i \tau B_{h}^{x}\right)+R_{j, l+1}\left(-i \tau B_{h}^{x}\right)^{j+l-1}}{2} \sum_{m=0}^{j} B_{m} f^{(m)}\left(t_{k-1}, x\right), \\
& f_{0}^{h}(x)=\tau \sum_{m=0}^{j+l-2} \widetilde{B}_{m} f^{(m)}(0, x)
\end{aligned}
$$

where

$$
\begin{gathered}
B_{m}=-\left(A_{h}^{x}\right)^{-1} \frac{\tau^{m-2}}{m !}+\left(A_{h}^{x}\right)^{-1} B_{m-2}, \\
2 \leq m \leq j+l-1, \\
B_{0}=\tau^{-2}\left(A_{h}^{x}\right)^{-1}\left(\frac{R_{j, l+1}\left(i \tau B_{h}^{x}\right)+R_{j, l+1}\left(-i \tau B_{h}^{x}\right)}{2}-I\right), \\
B_{1}=\tau^{-2}\left(A_{h}^{x}\right)^{-1} \\
\cdot\left(\left(B_{h}^{x}\right)^{-1} \frac{R_{j, l+1}\left(i \tau B_{h}^{x}\right)-R_{j, l+1}\left(-i \tau B_{h}^{x}\right)}{2 i}-\tau I\right), \\
\widetilde{B}_{m}=B_{m-1}, \quad 1 \leq m \leq j+l-1, \\
\widetilde{B}_{0}=\tau^{-2}\left(B_{h}^{x}\right)^{-1} \frac{R_{j, l+1}\left(i \tau B_{h}^{x}\right)-R_{j, l+1}\left(-i \tau B_{h}^{x}\right)}{2 i} .
\end{gathered}
$$

Theorem 74. Let $\tau$ and $|h|$ be sufficiently small numbers. Then the solutions of difference schemes (141) for $j=l$ and $j=l-1$ satisfy the following stability estimates:

$$
\begin{aligned}
& \max _{0 \leq k \leq N}\left\|u_{k}^{h}\right\|_{L_{2}\left(\bar{\Omega}_{h}\right)} \\
& \leq M_{1}\left[\max _{0 \leq k \leq N-1}\left\|\left(B_{h}^{x}\right)^{-1} f_{k}^{h}\right\|_{L_{2}\left(\bar{\Omega}_{h}\right)}\right. \\
& \left.+\left\|\left(B_{h}^{x}\right)^{-1} \psi^{h}\right\|_{L_{2}\left(\bar{\Omega}_{h}\right)}+\left\|\varphi^{h}\right\|_{L_{2}\left(\bar{\Omega}_{h}\right)}\right], \\
& \max _{0 \leq k \leq N} \sum_{r=1}^{n}\left\|\left(u_{k}^{h}\right)_{x_{r} r_{j}}\right\|_{L_{2}\left(\bar{\Omega}_{h}\right)} \\
& +\max _{1 \leq k \leq N}\left\|\tau^{-1}\left(u_{k}^{h}-u_{k-1}^{h}\right)\right\|_{L_{2}\left(\bar{\Omega}_{h}\right)} \\
& \leq M_{1}\left[\max _{0 \leq k \leq N-1}\left\|f_{k}^{h}\right\|_{L_{2}\left(\bar{\Omega}_{h}\right)}\right. \\
& \left.+\left\|\psi^{h}\right\|_{L_{2}\left(\bar{\Omega}_{h}\right)}+\sum_{r=1}^{n}\left\|\varphi_{\bar{x}_{r}, m_{r}}^{h}\right\|_{L_{2}\left(\bar{\Omega}_{h}\right)}\right], \\
& \max _{0 \leq k \leq N} \sum_{r=1}^{n}\left\|\left(u_{k}^{h}\right)_{\bar{x}_{r} x_{r} r_{j}}\right\|_{L_{2}\left(\bar{\Omega}_{h}\right)} \\
& +\max _{1 \leq k \leq N-1}\left\|\tau^{-2}\left(u_{k+1}^{h}-2 u_{k}^{h}+u_{k-1}^{h}\right)\right\|_{L_{2}\left(\bar{\Omega}_{h}\right)} \\
& \leq M_{1}\left[\max _{1 \leq k \leq N-1}\left\|\tau^{-1}\left(f_{k}^{h}-f_{k-1}^{h}\right)\right\|_{L_{2}\left(\bar{\Omega}_{h}\right)}\right. \\
& +\left\|f_{0}^{h}\right\|_{L_{2}\left(\bar{\Omega}_{h}\right)}+\sum_{r=1}^{n} \tau^{-1}\left\|\psi_{\bar{x}_{r}, m_{r}}^{h}\right\|_{L_{2}\left(\bar{\Omega}_{h}\right)} \\
& \left.+\sum_{r=1}^{n}\left\|\left(\varphi_{\bar{x}_{r}}^{h}\right)_{x_{r}, m_{r}}\right\|_{L_{2}\left(\bar{\Omega}_{h}\right)}\right] .
\end{aligned}
$$

Here $M_{1}$ does not depend on $\tau, h, \varphi^{h}(x), \psi^{h}(x)$, or $f_{k}^{h}(x), 0 \leq$ $k \leq N-1$.

Theorem 75. Let $\tau$ and $|h|$ be sufficiently small numbers. Then the solutions of difference schemes (141) for $j=l+1$ satisfy the following stability estimates:

$$
\begin{aligned}
& \max _{0 \leq k \leq N}\left\|u_{k}^{h}\right\|_{L_{2}\left(\bar{\Omega}_{h}\right)} \\
& \leq M_{1}\left[\max _{0 \leq k \leq N-1}\left\|f_{k}^{h}\right\|_{L_{2}\left(\bar{\Omega}_{h}\right)}\right. \\
& \\
&\left.+\left\|\psi^{h}\right\|_{L_{2}\left(\bar{\Omega}_{h}\right)}+\sum_{r=1}^{n}\left\|\varphi_{\bar{x}_{r}, m_{r}}^{h}\right\|_{L_{2}\left(\bar{\Omega}_{h}\right)}\right],
\end{aligned}
$$




$$
\begin{aligned}
\max _{0 \leq k \leq N} \sum_{r=1}^{n} \| & \left(u_{k}^{h}\right)_{x_{r} r_{j}} \|_{L_{2}\left(\bar{\Omega}_{h}\right)} \\
& +\max _{1 \leq k \leq N}\left\|\tau^{-1}\left(u_{k}^{h}-u_{k-1}^{h}\right)\right\|_{L_{2}\left(\bar{\Omega}_{h}\right)} \\
\leq & M_{1}\left[\max _{0 \leq k \leq N-1} \sum_{r=1}^{n}\left\|\left(f_{k}^{h}\right)_{\bar{x}_{r}, m_{r}}\right\|_{L_{2}\left(\bar{\Omega}_{h}\right)}\right. \\
& \left.+\sum_{r=1}^{n}\left\|\psi_{\bar{x}_{r}, m_{r}}^{h}\right\| L_{L_{2}\left(\bar{\Omega}_{h}\right)}+\sum_{r=1}^{n}\left\|\left(\varphi_{\bar{x}_{r}}^{h}\right)_{x_{r}, m_{r}}\right\|_{L_{2}\left(\bar{\Omega}_{h}\right)}\right] \\
& +\max _{0 \leq k \leq N} \sum_{r=1}^{n}\left\|\left(u_{k}^{h}\right)_{\bar{x}_{r} x_{r} r_{j}}\right\|_{L_{2}\left(\bar{\Omega}_{h}\right)}\left(u_{k+1}^{h}-2 u_{k}^{h}+u_{k-1}^{h}\right) \|_{L_{2}\left(\bar{\Omega}_{h}\right)} \\
\leq & M_{1}\left[\max _{1 \leq k \leq N-1} \tau^{-1} \sum_{r=1}^{n}\left\|\left(f_{k}^{h}\right)_{\bar{x}_{r}, m_{r}}-\left(f_{k-1}^{h}\right)_{\bar{x}_{r}, m_{r}}\right\|_{L_{2}\left(\bar{\Omega}_{h}\right)}\right. \\
& +\sum_{r=1}^{n}\left\|\left(f_{0}^{h}\right)_{\bar{x}_{r}, m_{r}}\right\|\left\|_{L_{2}\left(\bar{\Omega}_{h}\right)}+\sum_{r=1}^{n}\right\|\left(\psi_{\bar{x}_{r}}^{h}\right)_{x_{r}, m_{r}} \|_{L_{2}\left(\bar{\Omega}_{h}\right)} \\
& \left.+\sum_{r=1}^{n}\left\|\left(\left(\varphi_{\bar{x}_{r}}^{h}\right)_{x_{r}}\right)_{x_{r}, m_{r}}\right\|_{L_{2}\left(\bar{\Omega}_{h}\right)}\right]
\end{aligned}
$$

Here $M_{1}$ does not depend on $\tau, h, \varphi^{h}(x), \psi^{h}(x)$, or $f_{k}^{h}(x)$, $0 \leq k \leq N-1$.

The proofs of Theorems 74 and 75 are based on Theorems 72 and 73 and the symmetry property of the operator $A_{h}^{x}$ defined by formula (139) and the following theorem on the coercivity inequality for the solution of the elliptic difference problem in $L_{2 h}$.

Theorem 76 (see [116]). For the solutions of the elliptic difference problem

$$
\begin{gathered}
A_{h}^{x} u^{h}(x)=\omega^{h}(x), \quad x \in \Omega_{h}, \\
u^{h}(x)=0, \quad x \in S_{h},
\end{gathered}
$$

the following coercivity inequality holds:

$$
\sum_{r=1}^{n}\left\|u^{h}{ }_{x_{r} \bar{x}_{r}}\right\|_{L_{2 h}} \leq M\left\|\omega^{h}\right\|_{L_{2 h}},
$$

where $M$ does not depend on $h$ or $\omega^{h}$.

Note that in a similar manner one can construct the difference schemes of a high order of accuracy with respect to one variable for approximate solutions of boundary value problem (113). Abstract Theorems 71 and 72 permit us to obtain the stability estimates for solutions of these difference schemes.

Second, we consider the high order of accuracy two-step difference schemes generated by Taylor's decomposition on three points for the approximate solutions of initial value problem (109).
Theorem 77 (see [2]). Let the function $v(t)(0 \leq t \leq T)$ have a $(2 l+2 j+2)$ th continuous derivative and $t_{k-1}, t_{k}, t_{k+1} \in[0, T]_{\tau}$. Then, one has the following Taylor decomposition on the three points:

$$
\begin{aligned}
& v\left(t_{k+1}\right)-2 v\left(t_{k}\right)+v\left(t_{k-1}\right)-\sum_{s=1}^{l} \alpha_{s} v^{(2 s)}\left(t_{k}\right) \tau^{2 s} \\
& -\sum_{s=1}^{j} \eta_{s}\left\{v^{(2 s)}\left(t_{k-1}\right)+v^{(2 s)}\left(t_{k+1}\right)\right\} \tau^{2 s}=o\left(\tau^{2 l+2 j+2}\right),
\end{aligned}
$$

where $\eta_{m}, m=1, \ldots, j$, is the solution of system

$$
\begin{gathered}
\eta_{s}=\frac{1}{(2 s) !}-\sum_{m=1}^{s-1} \frac{\eta_{m}}{(2 s-2 m) !}, \\
\text { for any } s, \quad l+1 \leq s \leq j, \\
\sum_{m=1}^{j} \frac{(2 s) ! \eta_{m}}{(2 s-2 m) !}=1, \quad \text { for any } s, j+1 \leq s \leq l+j, \\
\alpha_{s}=\left(\frac{1}{(2 s) !}-\eta_{s}-\sum_{m=1}^{s-1} \eta_{m} \frac{1}{(2(s-m)) !}\right) 2 \\
\text { for any } s, \quad 2 \leq s \leq l .
\end{gathered}
$$

Suppose further that the function $v(t)(0 \leq t \leq T)$ has a $(2 l+2 j+1)$ th continuous derivative. Then, one has the following Taylor decomposition on two points:

$$
\begin{aligned}
v(\tau) & -v(0)+\sum_{s=1}^{2 j} \delta_{s} v^{(s)}(\tau) \tau^{s} \\
& -\sum_{s=1}^{2 l} \rho_{s} v^{(s)}(0) \tau^{s}=o\left(\tau^{2 l+2 j+1}\right),
\end{aligned}
$$

where

$$
\begin{gathered}
\rho_{s}=\frac{(2 l+2 j-s) !(2 l) !}{(2 l+2 j) ! s !(2 l-s) !} \quad \text { for any } s, 1 \leq s \leq 2 l, \\
\delta_{s}=\frac{(2 l+2 j-s) !(2 j) !(-1)^{s}}{(2 l+2 j) ! s !(2 j-s) !} \quad \text { for any } s, 1 \leq s \leq 2 j .
\end{gathered}
$$

Now, we will consider the applications of Taylor's decomposition (147) of the function $v(t)$ on the three points and Taylor's decomposition (149) of the function $v(t)$ on the two points to approximate solutions of initial value problem (109). From (147) and (149), it is clear that for the approximate solution of problem (109) it is necessary to find $v^{(j)}(\tau)$ for any $s, 1 \leq s \leq 2 l, v^{(2 s)}\left(t_{k}\right)$ for any $s, 1 \leq s \leq l$, and $v^{(2 s)}\left(t_{k-1}\right)$, $v^{(2 s)}\left(t_{k+1}\right)$ for any $s, 1 \leq s \leq j$. Using the equation

$$
v^{\prime \prime}(t)=-A v(t)+f(t),
$$


we obtain

$$
\begin{aligned}
v^{(2 n)}(t)= & (-A)^{n} v(t) \\
& +\sum_{\lambda=1}^{n}(-A)^{n-\lambda} f^{(2 \lambda-2)}(t), \quad n=2, \ldots, \\
v^{(2 n+1)}(t)= & (-A)^{n} v^{\prime}(t) \\
& +\sum_{\lambda=1}^{n}(-A)^{n-\lambda} f^{(2 \lambda-1)}(t), \quad n=1, \ldots
\end{aligned}
$$

Suppose further that the function $v(t)(0 \leq t \leq T)$ has a $(2 l+2 j-2 m+2)$ th continuous derivative. Then, we have the following Taylor decomposition on two points:

$$
-\sum_{s=1}^{2 j-2 m+2[m / 2]+1} b_{s} v^{(s)}(0) \tau^{s}=o\left(\tau^{2 l+2 j-2 m+2}\right)
$$

where

$$
\begin{gathered}
a_{s}=\frac{(2 l+2 j-2 m+1-s) !(2 l-2[m / 2]) !(-1)^{s}}{(2 l+2 j+1-2 m) ! s !(2 p-2[m / 2]-s) !} \\
\text { for any } s, \quad 1 \leq s \leq 2 l-2\left[\frac{m}{2}\right], \\
b_{s}=\frac{(2 l+2 j+1-2 m-s) !(2 j-2 m+2[m / 2]+1) !}{(2 l+2 j+1-2 m) ! s !(2 j-2 m+2[m / 2]+1-s) !} \\
\quad \text { for any } s, \quad 1 \leq s \leq 2 j-2 m+2\left[\frac{m}{2}\right]+1,
\end{gathered}
$$

where $[a]$ denotes the integer part of the number $a$. Further using formulas (152), (153), and (154), we can write

$$
\begin{aligned}
v^{\prime}(\tau) & -v^{\prime}(0)+\sum_{n=1}^{l-[m / 2]} a_{2 n}(-A)^{n} v^{\prime}(\tau) \tau^{2 n} \\
& +\sum_{n=1}^{l-[m / 2]} a_{2 n} \sum_{\lambda=1}^{n}(-A)^{n-\lambda} f^{(2 \lambda-1)}(\tau) \tau^{2 n} \\
& +\sum_{n=1}^{l-[m / 2]} a_{2 n-1}(-A)^{n} v(\tau) \tau^{2 n-1} \\
& +\sum_{n=1}^{l-[m / 2]} a_{2 n-1} \sum_{\lambda=1}^{n}(-A)^{n-\lambda} f^{(2 \lambda-2)}(\tau) \tau^{2 n-1}
\end{aligned}
$$

$$
\begin{aligned}
& -\sum_{n=1}^{j-m+[m / 2]} b_{2 n}(-A)^{n} v^{\prime}(0) \tau^{2 n} \\
& -\sum_{n=1}^{j-m+[m / 2]} b_{2 n} \sum_{\lambda=1}^{n}(-A)^{n-\lambda} f^{(2 \lambda-1)}(0) \tau^{2 n} \\
& -\sum_{n=1}^{j-m+[m / 2]+1} b_{2 n-1}(-A)^{n} v(0) \tau^{2 n-1} \\
& -\sum_{n=1}^{j-m+[m / 2]+1} b_{2 n-1} \sum_{\lambda=1}^{n}(-A)^{n-\lambda} f^{(2 \lambda-2)}(0) \tau^{2 n-1} \\
& =o\left(\tau^{2 l+2 j-2 m+2}\right), \quad 0 \leq m \leq j-1 .
\end{aligned}
$$

From the last formula it follows that

$$
\begin{aligned}
(I+ & \left.\sum_{n=1}^{l-[m / 2]} a_{2 n}(-A)^{n} \tau^{2 n}\right) v^{\prime}(\tau) \\
= & \left(I+\sum_{n=1}^{j-m+[m / 2]} b_{2 n}(-A)^{n} \tau^{2 n}\right) v^{\prime}(0) \\
& -\sum_{n=1}^{l-[m / 2]} a_{2 n-1}(-A)^{n} v(\tau) \tau^{2 n-1}
\end{aligned}
$$$$
+\sum_{n=1}^{j-m+[m / 2]+1} b_{2 n-1}(-A)^{n} v(0) \tau^{2 n-1}
$$$$
-\sum_{n=1}^{l-[m / 2]} a_{2 n} \sum_{\lambda=1}^{n}(-A)^{n-\lambda} f^{(2 \lambda-1)}(\tau) \tau^{2 n}
$$$$
-\sum_{n=1}^{l-[m / 2]} a_{2 n-1} \sum_{\lambda=1}^{n}(-A)^{n-\lambda} f^{(2 \lambda-2)}(\tau) \tau^{2 n-1}
$$$$
+\sum_{n=1}^{j-m+[m / 2]} b_{2 n} \sum_{\lambda=1}^{n}(-A)^{n-\lambda} f^{(2 \lambda-1)}(0) \tau^{2 n}
$$$$
+\sum_{n=1}^{j-m+[m / 2]+1} b_{2 n-1} \sum_{\lambda=1}^{n}(-A)^{n-\lambda} f^{(2 \lambda-2)}(0) \tau^{2 n-1}
$$$$
+o\left(\tau^{2 l+2 j-2 m+2}\right), \quad 0 \leq m \leq j-1 .
$$ 
Suppose further that the operator $\left(I+\sum_{n=1}^{l-[m / 2]} a_{2 n}(-A)^{n} \tau^{2 n}\right)$ has a bounded inverse. Then

$$
\begin{aligned}
& v^{\prime}(\tau)=\left(I+\sum_{n=1}^{l-[m / 2]} a_{2 n}(-A)^{n} \tau^{2 n}\right)^{-1} \\
& \cdot\left\{\left(I+\sum_{n=1}^{j-m+[m / 2]} b_{2 n}(-A)^{n} \tau^{2 n}\right) v^{\prime}(0)\right. \\
& -\sum_{n=1}^{l-[m / 2]} a_{2 n-1}(-A)^{n} v(\tau) \tau^{2 n-1} \\
& +\sum_{n=1}^{j-m+[m / 2]+1} b_{2 n-1}(-A)^{n} v(0) \tau^{2 n-1} \\
& -\sum_{n=1}^{l-[m / 2]} a_{2 n} \sum_{\lambda=1}^{n}(-A)^{n-\lambda} f^{(2 \lambda-1)}(\tau) \tau^{2 n} \\
& -\sum_{n=1}^{l-[m / 2]} a_{2 n-1} \sum_{\lambda=1}^{n}(-A)^{n-\lambda} f^{(2 \lambda-2)}(\tau) \tau^{2 n-1} \\
& +\sum_{n=1}^{j-m+[m / 2]} b_{2 n} \sum_{\lambda=1}^{n}(-A)^{n-\lambda} f^{(2 \lambda-1)}(0) \tau^{2 n} \\
& \left.+\sum_{n=1}^{j-m+[m / 2]+1} b_{2 n-1} \sum_{\lambda=1}^{n}(-A)^{n-\lambda} f^{(2 \lambda-2)}(0) \tau^{2 n-1}\right\} \\
& +o\left(\tau^{2 l+2 j-2 m+2}\right), \quad 0 \leq m \leq j-1 .
\end{aligned}
$$

Now, using formulas (147), (149), (152), (153), (154), and (158), we obtain the difference schemes of a $(2 l+2 j)$ th order of accuracy:

$$
\begin{gathered}
\frac{u_{k+1}-2 u_{k}+u_{k-1}}{\tau^{2}}-\sum_{s=1}^{l} \alpha_{s}(-A)^{s} \tau^{2 s-2} u_{k} \\
-\sum_{s=1}^{j} \eta_{s}(-A)^{s} \tau^{2 s-2}\left(u_{k-1}+u_{k+1}\right)=f_{k}^{j, l}, \\
f_{k}^{j, l}=\sum_{s=1}^{j} \eta_{s} \sum_{\lambda=1}^{s}(-A)^{s-\lambda} \\
+\sum_{s=1}^{l} \alpha_{s} \sum_{\lambda=1}^{s}(-A)^{s-\lambda} f^{(2 \lambda-2)}\left(t_{k}\right) \tau^{2 s-2}, \\
\quad 1 \leq k \leq N-1, \\
u_{0}=v_{0},
\end{gathered}
$$

$\frac{u_{1}-u_{0}}{\tau}-\sum_{n=1}^{l} \rho_{2 n}(-A)^{n} \tau^{2 n-1} v(0)$

$-\sum_{n=1}^{l} \rho_{2 n-1}(-A)^{n-1} \tau^{2 n-2} v^{\prime}(0)$

$+\sum_{n=1}^{j} \delta_{2 n}(-A)^{n} \tau^{2 n-1} u_{1}+\sum_{m=1}^{j} \delta_{2 m-1}(-A)^{m} \tau^{2 m-2}$

$\times\left(I+\sum_{n=1}^{l-[m / 2]} a_{2 n}(-A)^{n} \tau^{2 n}\right)^{-1}$

$\cdot\left\{\left(I+\sum_{n=1}^{j-m+[m / 2]} b_{2 n}(-A)^{n} \tau^{2 n}\right) v^{\prime}(0)\right.$

$-\sum_{n=1}^{l-[m / 2]} a_{2 n-1}(-A)^{n} \tau^{2 n-1} u_{1}$

$\left.+\sum_{n=1}^{j-m+[m / 2]+1} b_{2 n-1}(-A)^{n} \tau^{2 n-1} v(0)\right\}$

$=\sum_{n=1}^{l} \rho_{2 n}(-A)^{n} \tau^{2 n-1} \sum_{\lambda=1}^{n}(-A)^{n-\lambda} f^{(2 \lambda-2)}(0)$

$+\sum_{n=1}^{l} \rho_{2 n-1}(-A)^{n-1} \tau^{2 n-2}$

$\cdot \sum_{\lambda=1}^{n}(-A)^{n-\lambda} f^{(2 \lambda-1)}(0)$

$-\sum_{n=1}^{j} \delta_{2 n}(-A)^{n} \tau^{2 n-1}$

$\cdot \sum_{\lambda=1}^{n}(-A)^{n-\lambda} f^{(2 \lambda-2)}(\tau)$

$-\sum_{n=1}^{j} \delta_{2 n-1}(-A)^{n} \tau^{2 n-2}$

$\cdot \sum_{\lambda=1}^{n-1}(-A)^{n-\lambda} f^{(2 \lambda-1)}(\tau)$

$-\sum_{m=1}^{j} \delta_{2 m-1}(-A)^{m} \tau^{2 m-2}$

$\left(I+\sum_{n=1}^{l-[m / 2]} a_{2 n}(-A)^{n} \tau^{2 n}\right)^{-1}$

$\left(I+\sum_{n=1}^{j-m+[m / 2]} b_{2 n}(-A)^{n} \tau^{2 n}\right)$

$\cdot\left\{-\sum_{n=1}^{l-[m / 2]} a_{2 n} \sum_{\lambda=1}^{n}(-A)^{n-\lambda} f^{(2 \lambda-1)}(\tau) \tau^{2 n}\right.$

$-\sum_{n=1}^{l-[m / 2]} a_{2 n-1} \sum_{\lambda=1}^{n}(-A)^{n-\lambda} f^{(2 \lambda-2)}(\tau) \tau^{2 n-1}$ 


$$
\begin{aligned}
& +\sum_{n=1}^{j-m+[m / 2]} b_{2 n} \sum_{\lambda=1}^{n}(-A)^{n-\lambda} f^{(2 \lambda-1)}(0) \tau^{2 n} \\
& +\sum_{n=1}^{j-m+[m / 2]+1} b_{2 n-1} \\
& \left.\cdot \sum_{\lambda=1}^{n}(-A)^{n-\lambda} f^{(2 \lambda-2)}(0) \tau^{2 n-1}\right\}
\end{aligned}
$$

for the approximate solution of initial value problem (109). Suppose that the operator

$$
\begin{aligned}
(I+ & \sum_{n=1}^{j} \delta_{2 n}(-A)^{n} \tau^{2 n}-\sum_{m=1}^{j} \delta_{2 m-1}(-A)^{m} \tau^{2 m-1} \\
& \left.\cdot\left(I+\sum_{n=1}^{l-[m / 2]} a_{2 n}(-A)^{n} \tau^{2 n}\right)^{-1} \sum_{n=1}^{l-[m / 2]} a_{2 n-1}(-A)^{n} \tau^{2 n-1}\right)
\end{aligned}
$$

has a bounded inverse. Suppose further that the operator $(I-$ $\left.\sum_{s=1}^{j} \eta_{s}(-A)^{s} \tau^{2 s}\right)$ has a bounded inverse and

$$
\begin{aligned}
& I-\frac{1}{4}\left(2 I+\sum_{s=1}^{l} \alpha_{s}(-A)^{s} \tau^{2 s}\right)^{2}\left(I-\sum_{s=1}^{j} \eta_{s}(-A)^{s} \tau^{2 s}\right)^{-2} \\
& \quad \geq 0 .
\end{aligned}
$$

This problem is uniquely solvable and the following formula holds:

$$
\begin{aligned}
& u_{k}=\left(\widetilde{R}_{j, l}(-i \tau A)-\widetilde{R}_{j, l}(i \tau A)\right)^{-1} \widetilde{R}_{j, l}(i \tau A) \\
& \cdot \widetilde{R}_{j, l}(-i \tau A)\left(\widetilde{R}_{j, l}^{k-1}(i \tau A)-\widetilde{R}_{j, l}^{k-1}(-i \tau A)\right) u_{0} \\
&+\left(\widetilde{R}_{j, l}(-i \tau A)-\widetilde{R}_{j, l}(i \tau A)\right)^{-1} \\
& \cdot\left(\widetilde{R}_{j, l}^{k}(-i \tau A)-\widetilde{R}_{j, l}^{k}(i \tau A)\right) u_{1} \\
&+\sum_{j=1}^{k}\left(\widetilde{R}_{j, l}(-i \tau A)-\widetilde{R}_{j, l}(i \tau A)\right)_{j, l}^{-1} \widetilde{R}_{j, l}(i \tau A) \widetilde{R}_{j, l}(-i \tau A) \\
& \times\left(\widetilde{R}_{j, l}^{k-m}(-i \tau A)-\widetilde{R}_{j, l}^{k-m}(i \tau A)\right) \\
& \cdot\left(I-\sum_{s=1}^{j} \eta_{s}(-A)^{s} \tau^{2 s}\right)^{-1} f_{m}^{j, l}, \quad 2 \leq k \leq N, \\
& u_{1}=\widetilde{c}_{\tau} \varphi+\widetilde{s}_{\tau} \psi+\tau f_{0}^{j, l} .
\end{aligned}
$$

Here

$$
\begin{aligned}
& \widetilde{R}_{j, l}( \pm i \tau A) \\
& =\frac{1}{2}\left(2 I+\sum_{s=1}^{l} \alpha_{s}(-A)^{s} \tau^{2 s}\right)\left(I-\sum_{s=1}^{j} \eta_{s}(-A)^{s} \tau^{2 s}\right)^{-1} \\
& \pm i\left(I-\frac{1}{4}\left(2 I+\sum_{s=1}^{l} \alpha_{s}(-A)^{s} \tau^{2 s}\right)^{2}\right. \\
& \left.\cdot\left(I-\sum_{s=1}^{j} \eta_{s}(-A)^{s} \tau^{2 s}\right)^{-2}\right)^{1 / 2} \\
& \widetilde{c}_{\tau}=\left(I+\sum_{n=1}^{j} \delta_{2 n}(-A)^{n} \tau^{2 n}-\sum_{m=1}^{j} \delta_{2 m-1}(-A)^{m} \tau^{2 m-1}\right. \\
& \times\left(I+\sum_{n=1}^{l-[m / 2]} a_{2 n}(-A)^{n} \tau^{2 n}\right)^{-1} \\
& \left.\cdot \sum_{n=1}^{l-[m / 2]} a_{2 n-1}(-A)^{n} \tau^{2 n-1}\right)^{-1} \\
& \times\left\{\sum_{n=1}^{l} \rho_{2 n}(-A)^{n} \tau^{2 n-1}\right. \\
& +\sum_{m=1}^{j} \delta_{2 m-1}(-A)^{m} \tau^{2 m-2} \\
& \times\left(I+\sum_{n=1}^{l-[m / 2]} a_{2 n}(-A)^{n} \tau^{2 n}\right)^{-1} \\
& \left.\sum_{n=1}^{j-m+[m / 2]+1} b_{2 n-1}(-A)^{n} \tau^{2 n-1}\right\} \text {, } \\
& \tilde{\boldsymbol{s}}_{\tau}=\left(I+\sum_{n=1}^{j} \delta_{2 n}(-A)^{n} \tau^{2 n}\right. \\
& -\sum_{m=1}^{j} \delta_{2 m-1}(-A)^{m} \tau^{2 m-1} \\
& \times\left(I+\sum_{n=1}^{l-[m / 2]} a_{2 n}(-A)^{n} \tau^{2 n}\right)^{-1} \\
& \left.\cdot \sum_{n=1}^{l-[m / 2]} a_{2 n-1}(-A)^{n} \tau^{2 n-1}\right)^{-1} \\
& \times\left\{\sum_{n=1}^{l} \rho_{2 n-1}(-A)^{n-1} \tau^{2 n-2}\right.
\end{aligned}
$$




$$
\begin{aligned}
& +\sum_{m=1}^{j} \delta_{2 m-1}(-A)^{m} \tau^{2 m-2} \\
& \times\left(I+\sum_{n=1}^{l-[m / 2]} a_{2 n}(-A)^{n} \tau^{2 n}\right)^{-1} \\
& \left.\cdot\left(I+\sum_{n=1}^{j-m+[m / 2]} b_{2 n}(-A)^{n} \tau^{2 n}\right)\right\}, \\
& f_{0}^{j, l}=\sum_{n=1}^{l} \rho_{2 n}(-A)^{n} \tau^{2 n-1} \sum_{\lambda=1}^{n}(-A)^{n-\lambda} f^{(2 \lambda-2)}(0) \\
& +\sum_{n=1}^{l} \rho_{2 n-1}(-A)^{n-1} \tau^{2 n-2} \sum_{\lambda=1}^{n}(-A)^{n-\lambda} f^{(2 \lambda-1)}(0) \\
& -\sum_{n=1}^{j} \delta_{2 n}(-A)^{n} \tau^{2 n-1} \sum_{\lambda=1}^{n}(-A)^{n-\lambda} f^{(2 \lambda-2)}(\tau) \\
& -\sum_{n=1}^{j} \delta_{2 n-1}(-A)^{n} \tau^{2 n-2} \sum_{\lambda=1}^{n-1}(-A)^{n-\lambda} f^{(2 \lambda-1)}(\tau) \\
& -\sum_{m=1}^{j} \delta_{2 m-1}(-A)^{m} \tau^{2 m-2}\left(I+\sum_{n=1}^{l-[m / 2]} a_{2 n}(-A)^{n} \tau^{2 n}\right)^{-1} \\
& \cdot\left(I+\sum_{n=1}^{j-m+[m / 2]} b_{2 n}(-A)^{n} \tau^{2 n}\right) \\
& \left\{-\sum_{n=1}^{l-[m / 2]} a_{2 n} \sum_{\lambda=1}^{n}(-A)^{n-\lambda} f^{(2 \lambda-1)}(\tau) \tau^{2 n}\right. \\
& -\sum_{n=1}^{l-[m / 2]} a_{2 n-1} \sum_{\lambda=1}^{n}(-A)^{n-\lambda} f^{(2 \lambda-2)}(\tau) \tau^{2 n-1} \\
& +\sum_{n=1}^{j-m+[m / 2]} b_{2 n} \sum_{\lambda=1}^{n}(-A)^{n-\lambda} f^{(2 \lambda-1)}(0) \tau^{2 n} \\
& \left.+\sum_{n=1}^{j-m+[m / 2]+1} b_{2 n-1} \sum_{\lambda=1}^{n}(-A)^{n-\lambda} f^{(2 \lambda-2)}(0) \tau^{2 n-1}\right\} .
\end{aligned}
$$

From formula (162), it follows that the investigation of the stability of difference schemes (159) relies in an essential manner on a number of properties of the powers of the operator $\widetilde{R}_{j, l}( \pm i \tau A)$. We were not able to obtain the estimates for powers of the operator $\widetilde{R}_{j, l}( \pm i \tau A)$ in the general cases of numbers $j$ and $l$.
Theorem 78 (see [2]). Suppose that $u_{0} \in D(A), u_{1}-u_{0} \in$ $D\left(A^{1 / 2}\right)$. Then for the solution of two-step difference schemes (159) for $l=0$ the following stability inequalities hold:

$$
\begin{aligned}
& \max _{0 \leq k \leq N}\left\|u_{k}\right\|_{H} \\
& \leq M \max _{1 \leq k \leq N-1}\left\|A^{-1} f_{k}^{j, l}\right\|_{H} \\
&\left.+\left\|A^{-1 / 2} \tau^{-1}\left(u_{1}-u_{0}\right)\right\|_{H}+\left\|u_{0}\right\|_{H}\right], \\
& \max _{1 \leq k \leq N}\left\|\tau^{-1}\left(u_{k}-u_{k-1}\right)\right\|_{H} \\
& \leq M\left[\max _{1 \leq k \leq N-1}\left\|f_{k}^{j, l}\right\|_{H}\right. \\
&\left.\quad+\left\|\tau^{-1}\left(u_{1}-u_{0}\right)\right\|_{H}+\left\|A^{1 / 2} u_{0}\right\|_{H}\right], \\
& \max _{1 \leq k \leq N-1}\left\|\tau^{-2}\left(u_{k+1}-2 u_{k}+u_{k-1}\right)\right\|_{H} \\
& \leq M\left[\max _{2 \leq k \leq N-1}\left\|\tau^{-1}\left(f_{k}^{j, l}-f_{k-1}^{j, l}\right)\right\|_{H}+\left\|f_{1}^{j, l}\right\|_{H}\right. \\
&\left.\quad+\left\|A^{1 / 2} \tau^{-1}\left(u_{1}-u_{0}\right)\right\|_{H}+\left\|A u_{0}\right\|_{H}\right],
\end{aligned}
$$

where $M$ does not depend on $\tau, f_{k}^{j, l}, 1 \leq k \leq N-1$, or $u_{0}, u_{1}$.

Note that the assumptions of Theorem 78 actually hold in the general cases of numbers $j$ and $l$ under the following assumption:

$$
\tau\|A\|_{H \rightarrow H} \longrightarrow 0 \quad \text { when } \tau \longrightarrow 0 .
$$

Now, abstract Theorem 78 is applied in the investigation of difference schemes of higher order of accuracy with respect to one variable for approximate solutions of mixed boundary value problem (115). The first step of discretization of problem (115) is given above. Suppose that the operator

$$
\begin{aligned}
(I+ & \sum_{n=1}^{j} \delta_{2 n}(-A)^{n} \tau^{2 n}-\sum_{m=1}^{j} \delta_{2 m-1}(-A)^{m} \tau^{2 m-1} \\
& \cdot\left(I+\sum_{n=1}^{l-[m / 2]} a_{2 n}(-A)^{n} \tau^{2 n}\right)^{-1} \\
& \left.\cdot \sum_{n=1}^{l-[m / 2]} a_{2 n-1}(-A)^{n} \tau^{2 n-1}\right)
\end{aligned}
$$

has a bounded inverse. Suppose further that the operator ( $I-$ $\left.\sum_{s=1}^{j} \eta_{s}\left(-A_{h}^{x}\right)^{s} \tau^{2 s}\right)$ has a bounded inverse and

$$
\begin{aligned}
I-\frac{1}{4} & \left(2 I+\sum_{s=1}^{l} \alpha_{s}\left(-A_{h}^{x}\right)^{s} \tau^{2 s}\right)^{2} \\
& \left(I-\sum_{s=1}^{j} \eta_{s}\left(-A_{h}^{x}\right)^{s} \tau^{2 s}\right)^{-2} \geq 0 .
\end{aligned}
$$


Then in the second step we replace problem (140) with difference schemes (159):

$$
\begin{aligned}
& \tau^{-2}\left(u_{k+1}^{h}(x)-2 u_{k}^{h}(x)+u_{k-1}^{h}(x)\right)+\widetilde{A_{h}^{x}} u_{k}^{h}(x) \\
& +\widetilde{A A_{h}^{x}}\left(u_{k+1}^{h}(x)+u_{k-1}^{h}(x)\right)=f_{k}^{h}(x), \\
& t_{k}=k \tau, \quad 1 \leq k \leq N-1, x \in \Omega_{h}, \\
& \widetilde{A_{h}^{x}}=-\sum_{s=1}^{l} \alpha_{s}\left(-A_{h}^{x}\right)^{s} \tau^{2 s-2} \\
& \widetilde{A A_{h}^{x}}=-\sum_{s=1}^{j} \eta_{s}\left(-A_{h}^{x}\right)^{s} \tau^{2 s-2} \\
& f_{k}^{h}(x)=\sum_{s=1}^{j} \eta_{s} \sum_{\lambda=1}^{s}\left(-A_{h}^{x}\right)^{s-\lambda} \\
& \cdot\left(f^{(2 \lambda-2)}\left(t_{k+1}, x\right)+f^{(2 \lambda-2)}\left(t_{k-1}, x\right)\right) \tau^{2 s-2} \\
& +\sum_{s=1}^{l} \alpha_{i} \sum_{\lambda=1}^{s}\left(-A_{h}^{x}\right)^{s-\lambda} f^{(2 \lambda-2)}\left(t_{k}, x\right) \tau^{2 s-2} \\
& 1 \leq k \leq N-1 \\
& u_{1}^{h}(x)=\widetilde{c}_{\tau} \varphi^{h}(x)+\widetilde{s}_{\tau} \psi^{h}(x)+\tau f_{0}^{h}(x), \quad x \in \Omega_{h}, \\
& \widetilde{\mathcal{c}}_{\tau}=\left(I+\sum_{n=1}^{j} \delta_{2 n}\left(-A_{h}^{x}\right)^{n} \tau^{2 n}-\sum_{m=1}^{j} \delta_{2 m-1}\left(-A_{h}^{x}\right)^{m} \tau^{2 m-1}\right. \\
& \times\left(I+\sum_{n=1}^{l-[m / 2]} a_{2 n}\left(-A_{h}^{x}\right)^{n} \tau^{2 n}\right)^{-1} \\
& \left.\cdot \sum_{n=1}^{l-[m / 2]} a_{2 n-1}\left(-A_{h}^{x}\right)^{n} \tau^{2 n-1}\right)^{-1} \\
& \times\left\{\sum_{n=1}^{l} \rho_{2 n}\left(-A_{h}^{x}\right)^{n} \tau^{2 n-1}+\sum_{m=1}^{j} \delta_{2 m-1}\left(-A_{h}^{x}\right)^{m} \tau^{2 m-2}\right. \\
& \times\left(I+\sum_{n=1}^{l-[m / 2]} a_{2 n}\left(-A_{h}^{x}\right)^{n} \tau^{2 n}\right)^{-1} \\
& \left.\sum_{n=1}^{j-m+[m / 2]+1} b_{2 n-1}\left(-A_{h}^{x}\right)^{n} \tau^{2 n-1}\right\}, \\
& \widetilde{\boldsymbol{s}}_{\tau}=\left(I+\sum_{n=1}^{j} \delta_{2 n}\left(-A_{h}^{x}\right)^{n} \tau^{2 n}-\sum_{m=1}^{j} \delta_{2 m-1}\left(-A_{h}^{x}\right)^{m} \tau^{2 m-1}\right. \\
& \times\left(I+\sum_{n=1}^{l-[m / 2]} a_{2 n}\left(-A_{h}^{x}\right)^{n} \tau^{2 n}\right)^{-1}
\end{aligned}
$$

$$
\begin{gathered}
\left.\cdot \sum_{n=1}^{l-[m / 2]} a_{2 n-1}\left(-A_{h}^{x}\right)^{n} \tau^{2 n-1}\right)^{-1} \\
\times\left\{\sum_{n=1}^{l} \rho_{2 n-1}\left(-A_{h}^{x}\right)^{n-1} \tau^{2 n-2}\right. \\
+\sum_{m=1}^{j} \delta_{2 m-1}\left(-A_{h}^{x}\right)^{m} \tau^{2 m-2} \\
\times\left(I+\sum_{n=1}^{l-[m / 2]} a_{2 n}\left(-A_{h}^{x}\right)^{n} \tau^{2 n}\right)^{-1} \\
\left.\cdot\left(I+\sum_{n=1}^{j-m+[m / 2]} b_{2 n}\left(-A_{h}^{x}\right)^{n} \tau^{2 n}\right)\right\}
\end{gathered}
$$

$f_{0}^{h}(x)$

$$
\begin{aligned}
& =\sum_{n=1}^{l} \rho_{2 n}\left(-A_{h}^{x}\right)^{n} \tau^{2 n-1} \\
& \cdot \sum_{\lambda=1}^{n}\left(-A_{h}^{x}\right)^{n-\lambda} f^{(2 \lambda-2)}(0, x)+\sum_{n=1}^{l} \rho_{2 n-1}\left(-A_{h}^{x}\right)^{n-1} \tau^{2 n-2} \\
& \cdot \sum_{\lambda=1}^{n}\left(-A_{h}^{x}\right)^{n-\lambda} f^{(2 \lambda-1)}(0, x)-\sum_{n=1}^{j} \delta_{2 n}\left(-A_{h}^{x}\right)^{n} \tau^{2 n-1} \\
& \cdot \sum_{\lambda=1}^{n}\left(-A_{h}^{x}\right)^{n-\lambda} f^{(2 \lambda-2)}(\tau, x)-\sum_{n=1}^{j} \delta_{2 n-1}\left(-A_{h}^{x}\right)^{n} \tau^{2 n-2} \\
& \cdot \sum_{\lambda=1}^{n-1}\left(-A_{h}^{x}\right)^{n-\lambda} f^{(2 \lambda-1)}(\tau, x)-\sum_{m=1}^{j} \delta_{2 m-1}\left(-A_{h}^{x}\right)^{m} \tau^{2 m-2} \\
& \cdot\left(I+\sum_{n=1}^{l-[m / 2]} a_{2 n}\left(-A_{h}^{x}\right)^{n} \tau^{2 n}\right)^{-1} \\
& \times\left(I+\sum_{n=1}^{j-m+[m / 2]} b_{2 n}\left(-A_{h}^{x}\right)^{n} \tau^{2 n}\right) \\
& \left\{-\sum_{n=1}^{l-[m / 2]} a_{2 n} \sum_{\lambda=1}^{n}\left(-A_{h}^{x}\right)^{n-\lambda} f^{(2 \lambda-1)}(\tau, x) \tau^{2 n}\right. \\
& -\sum_{n=1}^{l-[m / 2]} a_{2 n-1} \sum_{\lambda=1}^{n}\left(-A_{h}^{x}\right)^{n-\lambda} f^{(2 \lambda-2)}(\tau, x) \tau^{2 n-1} \\
& +\sum_{n=1}^{j-m+[m / 2]} b_{2 n} \sum_{\lambda=1}^{n}\left(-A_{h}^{x}\right)^{n-\lambda} f^{(2 \lambda-1)}(0, x) \tau^{2 n} \\
& \left.+\sum_{n=1}^{j-m+[m / 2]+1} b_{2 n-1} \sum_{\lambda=1}^{n}\left(-A_{h}^{x}\right)^{n-\lambda} f^{(2 \lambda-2)}(0, x) \tau^{2 n-1}\right\} \text {. }
\end{aligned}
$$

We have the following. 
Theorem 79 (see [2]). Let $\tau$ and $|h|$ be sufficiently small numbers. Then the solutions of difference schemes (168) for $l=0$ satisfy the following stability estimates:

$$
\begin{gathered}
\max _{0 \leq k \leq N}\left\|u_{k}^{h}\right\|_{L_{2}\left(\bar{\Omega}_{h}\right)} \\
\leq M_{1}\left[\max _{0 \leq k \leq N-1}\left\|\left(B_{h}^{x}\right)^{-1} f_{k}^{h}\right\|_{L_{2}\left(\bar{\Omega}_{h}\right)}\right. \\
\left.+\left\|\left(B_{h}^{x}\right)^{-1} \psi^{h}\right\|_{L_{2}\left(\bar{\Omega}_{h}\right)}+\left\|\varphi^{h}\right\|_{L_{2}\left(\bar{\Omega}_{h}\right)}\right], \\
\max _{0 \leq k \leq N} \sum_{r=1}^{m}\left\|\left(u_{k}^{h}\right)_{x_{r} r_{j}}\right\|_{L_{2}\left(\bar{\Omega}_{h}\right)}+\max _{1 \leq k \leq N}\left\|\tau^{-1}\left(u_{k}^{h}-u_{k-1}^{h}\right)\right\|_{L_{2}\left(\bar{\Omega}_{h}\right)} \\
\leq M_{1}\left[\max _{0 \leq k \leq N-1}\left\|f_{k}^{h}\right\|_{L_{2}\left(\bar{\Omega}_{h}\right)}\right. \\
\left.+\left\|\psi^{h}\right\|_{L_{2}\left(\bar{\Omega}_{h}\right)}+\sum_{r=1}^{n}\left\|\varphi_{\bar{x}_{r}, m_{r}}^{h}\right\|_{L_{2}\left(\bar{\Omega}_{h}\right)}\right], \\
\max _{0 \leq k \leq N} \sum_{r=1}^{n}\left\|\left(u_{k}^{h}\right)_{\bar{x}_{r} x_{r} r_{j}}\right\|_{L_{2}\left(\bar{\Omega}_{h}\right)} \\
+\max _{1 \leq k \leq N-1}\left\|\tau^{-2}\left(u_{k+1}^{h}-2 u_{k}^{h}+u_{k-1}^{h}\right)\right\|_{L_{2}\left(\bar{\Omega}_{h}\right)} \\
\leq M_{1}\left[\max _{1 \leq k \leq N-1}\left\|\tau^{-1}\left(f_{k}^{h}-f_{k-1}^{h}\right)\right\|_{L_{2}\left(\bar{\Omega}_{h}\right)}+\left\|f_{0}^{h}\right\|_{L_{2}\left(\bar{\Omega}_{h}\right)}\right. \\
\left.+\sum_{r=1}^{n}\left\|\psi_{\bar{x}_{r}, m_{r}}^{h}\right\|_{L_{2}\left(\bar{\Omega}_{h}\right)}+\sum_{r=1}^{n}\left\|\left(\varphi_{\bar{x}_{r}}^{h}\right)_{x_{r}, m_{r}}\right\|_{L_{2}\left(\bar{\Omega}_{h}\right)}\right] .
\end{gathered}
$$

Here $M_{1}$ does not depend on $\tau, h, \varphi^{h}(x), \psi^{h}(x)$, or $f_{k}^{h}(x), 0 \leq$ $k \leq N-1$.

The proof of Theorem 79 is based on Theorem 78 and the symmetry property of the operator $A_{h}^{x}$ defined by formula (139) and Theorem 76 on the coercivity inequality for the solution of the elliptic difference problem in $L_{2 h}$.

In a similar manner one can construct the difference schemes of a high order of accuracy with respect to one variable for approximate solutions of boundary value problem (113). Abstract Theorem 78 permits us to obtain the stability estimates for the solutions of these difference schemes.

Note that most high orders of accuracy absolutely stable difference schemes for approximate solutions of problem (109) were generated by square roots of $A$. This action is very difficult to accomplish. Therefore, in spite of theoretical results, the role of their application to a numerical solution for an initial value problem is not great. Finally, in $[117,118]$, the third order of accuracy difference scheme

$$
\begin{aligned}
& \tau^{-2}\left(u_{k+1}-2 u_{k}+u_{k-1}\right) \\
&+\frac{2}{3} A u_{k}+\frac{1}{6} A\left(u_{k+1}+u_{k-1}\right)+\frac{1}{12} \tau^{2} A^{2} u_{k+1} \\
&= f_{k}, \\
& f_{k}= \frac{2}{3} f\left(t_{k}\right)+\frac{1}{6}\left(f\left(t_{k+1}\right)+f\left(t_{k-1}\right)\right) \\
&-\frac{1}{12} \tau^{2}\left(-A f\left(t_{k+1}\right)+f^{\prime \prime}\left(t_{k+1}\right)\right), \\
& 1 \leq k \leq N-1, \\
& u_{0}=\varphi, \\
&\left(I+\frac{\tau^{2}}{12} A+\frac{\tau^{4}}{144} A^{2}\right) \tau^{-1}\left(u_{1}-u_{0}\right) \\
&=-\frac{\tau}{2} A \varphi+\left(I-\frac{\tau^{2}}{12} A\right) \psi+f_{1,1} \tau, \\
& f_{1,1}=f(0)+\left(-f(0)+\tau f^{\prime}(0)\right) \frac{1}{2}-2 f^{\prime}(0) \frac{\tau}{6}
\end{aligned}
$$

and the fourth order of accuracy difference scheme

$$
\begin{aligned}
& \tau^{-2}\left(u_{k+1}-2 u_{k}+u_{k-1}\right)+\frac{5}{6} A u_{k} \\
& \quad+\frac{1}{12} A\left(u_{k+1}+u_{k-1}\right)-\frac{\tau^{2}}{72} A^{2} u_{k} \\
& \quad+\frac{\tau^{2}}{144} A^{2}\left(u_{k+1}+u_{k-1}\right)=f_{k}, \\
& f_{k}=\frac{5}{6} f\left(t_{k}\right)+\frac{1}{12}\left(f\left(t_{k+1}\right)+f\left(t_{k-1}\right)\right) \\
& +\frac{\tau^{2}}{72}\left(-A f\left(t_{k}\right)+f^{\prime \prime}\left(t_{k}\right)\right)-\frac{1}{144} \tau^{2} \\
& \quad \cdot\left(-A\left(f\left(t_{k+1}\right)+f\left(t_{k-1}\right)\right)+f^{\prime \prime}\left(t_{k+1}\right)+f^{\prime \prime}\left(t_{k-1}\right)\right), \\
& t_{k}=k \tau, \quad N \tau=1,
\end{aligned}
$$




$$
\begin{gathered}
u_{0}=\varphi, \\
\left(I+\frac{\tau^{2} A}{12}+\frac{\tau^{4} A^{2}}{144}\right) \tau^{-1}\left(u_{1}-u_{0}\right) \\
=-\frac{\tau}{2} A \varphi+\left(I-\frac{\tau^{2} A}{12}\right) \psi+f_{2,2} \tau, \\
f_{2,2}=\left\{\left(I-\frac{\tau^{2} A}{12}\right) f(0)\right. \\
+\left(-\left(I-\frac{5 \tau^{2} A}{12}\right) f(0)+\tau f^{\prime}(0)\right) \frac{1}{2} \\
+\left(-A \tau f(0)-2 f^{\prime}(0)+\tau f^{\prime \prime}(0)\right) \frac{\tau}{6} \\
\left.+\left(A f(0)-3 f^{\prime \prime}(0)\right) \frac{\tau^{2}}{24}\right\}
\end{gathered}
$$

for the approximate solution of initial value problem (109) generated by the integer powers of the operator $A$ were presented.

Theorem 80. Let $\varphi \in D(A), \psi \in D\left(A^{1 / 2}\right)$, and $f_{1,1} \in$ $D\left(A^{1 / 2}\right)$. Then, for the solution of difference scheme (170), the following stability estimates hold:

$$
\begin{aligned}
& \max _{1 \leq k \leq N}\left\|u_{k}\right\|_{H} \\
& \leq M\left\{\sum_{s=1}^{N-1}\left\|A^{-1 / 2} f_{s}\right\|_{H} \tau+\|\varphi\|_{H}\left\|A^{-1 / 2} \psi\right\|_{H}\right. \\
& \left.+\tau\left\|A^{-1 / 2} f_{1,1}\right\|_{H}\right\}, \\
& \max _{1 \leq k \leq N}\left\|A^{1 / 2} u_{k}\right\|_{H}+\max _{1 \leq k \leq N}\left\|\frac{u_{k}-u_{k-1}}{\tau}\right\|_{H} \\
& \leq M\left\{\sum_{s=1}^{N-1}\left\|f_{s}\right\|_{H} \tau+\left\|A^{1 / 2} \varphi\right\|_{H}+\|\psi\|_{H}+\tau\left\|f_{1,1}\right\|_{H}\right\}, \\
& \max _{1 \leq k \leq N}\left\|A u_{k}\right\|_{H}+\max _{1 \leq k \leq N}\left\|A^{1 / 2} \frac{u_{k}-u_{k-1}}{\tau}\right\|_{H} \\
& +\max _{1 \leq k \leq N-1}\left\|\frac{u_{k+1}-2 u_{k}+u_{k-1}}{\tau^{2}}\right\|_{H} \\
& \leq M\left\{\sum_{s=2}^{N-1}\left\|f_{s}-f_{s-1}\right\|_{H}+\left\|f_{1}\right\|_{H}\right. \\
& \left.+\|A \varphi\|_{H}+\left\|A^{1 / 2} \psi\right\|_{H}+\tau\left\|A^{1 / 2} f_{1,1}\right\|_{H}\right\},
\end{aligned}
$$

where $M$ does not depend on $\tau, \varphi, \psi, f_{1,1}$, or $f_{s}, 1 \leq s \leq N-1$.
Theorem 81. Let $\varphi \in D(A), \psi \in D\left(A^{1 / 2}\right)$, and $f_{2,2} \in D\left(A^{1 / 2}\right)$. Then, for the solution of difference scheme (171), the following stability estimates hold:

$$
\begin{aligned}
& \max _{1 \leq k \leq N}\left\|\frac{u_{k}+u_{k-1}}{2}\right\|_{H}\left\{\sum_{s=1}^{N-1}\left\|A^{-1 / 2} f_{s}\right\|_{H} \tau+\|\varphi\|_{H}\left\|A^{-1 / 2} \psi\right\|_{H}\right. \\
& \leq\left.+\tau\left\|A^{-1 / 2} f_{2,2}\right\|_{H}\right\}, \\
& \max _{1 \leq k \leq N}\left\|A^{1 / 2} \frac{u_{k}+u_{k-1}}{2}\right\|_{H}+\max _{1 \leq k \leq N-1}\left\|\frac{u_{k+1}-u_{k-1}}{2 \tau}\right\|_{H} \\
& \leq M\left\{\sum_{s=1}^{N-1}\left\|f_{s}\right\|_{H} \tau+\left\|A^{1 / 2} \varphi\right\|_{H}+\|\psi\|_{H}+\tau\left\|_{2,2}\right\|_{H}\right\}, \\
& \max _{1 \leq k \leq N}\left\|A \frac{u_{k}+u_{k-1}}{2}\right\|_{H}+\max _{1 \leq k \leq N}\left\|A^{1 / 2} \frac{u_{k+1}-u_{k-1}}{2 \tau}\right\|_{H} \\
&+\max _{1 \leq k \leq N-1}\left\|\frac{u_{k+1}-2 u_{k}+u_{k-1}}{\tau^{2}}\right\|_{H} \\
& \leq M\left\{\sum_{s=2}^{N-1}\left\|f_{s}-f_{s-1}\right\|_{H}+\left\|f_{1}\right\|_{H}\right. \\
&\left.+\|A \varphi\|_{H}+\left\|A^{1 / 2} \psi\right\|_{H}+\tau\left\|A^{1 / 2} f_{2,2}\right\|_{H}\right\},
\end{aligned}
$$

where $M$ does not depend on $\tau, \varphi, \psi, f_{1,1}$, or $f_{s}, 1 \leq s \leq N-1$.

Note that in a similar manner one can construct the difference schemes of third and fourth order of accuracy with respect to one variable for approximate solutions of boundary value problems (113) and (115). Abstract Theorems 80 and 81 permit us to obtain the stability estimates for the solutions of these difference schemes. A finite difference method and some results of numerical experiments are presented in order to support theoretical statements.

Note that we have not been able to obtain a sharp estimate for the constants figuring in the stability inequality. In $[66,67]$, numerical experiments of the initial-boundary value problem for the wave equation with nonhomogeneous cylindrical shells and for the one-dimensional hyperbolic partial differential with variable coefficients were proposed to obtain the constants figuring in the stability inequality.

Finally, in [119], the boundary value problems for $2 k$ th order partial differential equations were investigated. Wellposedness of two boundary value problems for partial differential equations in the cases of even and odd $k$ was established. Note that solvability results were dependent on the evenness and oddness of the number $k$. 
7.2. A Cauchy Problem with $t$-Dependent $A(t)$ Operators Coefficients. We consider the abstract Cauchy problem for the hyperbolic equations

$$
\begin{gathered}
v^{\prime \prime}(t)+A(t) v(t)=f(t) \quad(0 \leq t \leq T) ; \\
v(0)=\varphi, \quad v^{\prime}(0)=\psi
\end{gathered}
$$

in Hilbert space $H$ with the self-adjoint positive definite operators $A(t)$ in $H$ with $t$-independent domain $D=$ $D(A(t))$.

Function $v(t)$ is called a solution of problem (174) if the following conditions are satisfied:

(i) $v(t)$ is twice continuously differentiable on the segment $[0, T]$.

(ii) The element $v(t)$ belongs to $D$ for all $t \in[0, T]$ and the function $A(t) v(t)$ is continuous on the segment $[0, T]$.

(iii) $v(t)$ satisfies the equation and initial conditions (174).

Of great interest is the study of absolutely stable difference schemes of a high order of accuracy for hyperbolic partial differential equations. Such type of stability inequalities for the solutions of the first order of accuracy difference scheme

$$
\begin{gathered}
\tau^{-2}\left(u_{k+1}-2 u_{k}+u_{k-1}\right)+A_{k} u_{k+1}=f_{k}, \\
A_{k}=A\left(t_{k}\right), \quad f_{k}=f\left(t_{k}\right), \\
t_{k}=k \tau, \quad 1 \leq k \leq N-1, \\
\tau^{-1}\left(u_{1}-u_{0}\right)+i A_{1}^{1 / 2} u_{1}=i A_{0}^{1 / 2} u_{0}+\psi, \\
u_{0}=\varphi
\end{gathered}
$$

for approximately solving problem (174) has been established for the first time in [62]. The following theorems summarize Sobolevskii and Chebotaryeva's results.

Theorem 82 (see [62]). Assume that the operator-function $A^{1 / 2}(t) A^{-1 / 2}(0)$ has a finite variation on the segment $[0, T]$ and

$$
\left\|A^{1 / 2}(0) A^{-1 / 2}(t)\right\| \leq M_{1 / 2}
$$

for any $t \in[0, T], \varphi \in D(A(0))$, and $\psi \in D\left(A^{1 / 2}(0)\right)$. Then, the following estimate

$$
\begin{gathered}
\max _{1 \leq k \leq N}\left\|\frac{u_{k}-u_{k-1}}{\tau}\right\|_{H}+\max _{1 \leq k \leq N}\left\|A^{1 / 2}(0) u_{k}\right\|_{H}+\max _{0 \leq k \leq N}\left\|u_{k}\right\|_{H} \\
\quad \leq M_{1}\left[\left\|A^{1 / 2}(0) \varphi\right\|_{H}+\|\psi\|_{H}+\sum_{s=1}^{N-1}\left\|f_{s}\right\|_{H} \tau\right]
\end{gathered}
$$

holds, where $M_{1}$ does not depend on $\varphi, \psi, f_{s}(0 \leq s \leq N-1)$, or $\tau$.

Theorem 83 (see [62]). Assume that the operator-function $A(t) A^{-1}(0)$ has a finite variation on the segment $[0, T]$ and

$$
\left\|A(0) A^{-1}(t)\right\| \leq M_{1}
$$

for any $t \in[0, T], \varphi \in D(A(0))$, and $\psi \in D\left(A^{1 / 2}(0)\right)$. Then, the following estimate

$$
\begin{gathered}
\max _{1 \leq k \leq N}\left\|A^{1 / 2}(0) \frac{u_{k}-u_{k-1}}{\tau}\right\|_{H}+\max _{1 \leq k \leq N}\left\|A(0) u_{k}\right\|_{H} \\
+\max _{1 \leq k \leq N-1}\left\|\tau^{-2}\left(u_{k+1}-2 u_{k}+u_{k-1}\right)\right\|_{H} \\
\leq M_{2}\left[\|A(0) \varphi\|_{H}+\left\|A^{1 / 2}(0) \psi\right\|_{H}\right. \\
\left.+\left\|f_{1}\right\|_{H}+\sum_{s=2}^{N-1}\left\|f_{s}-f_{s-1}\right\|_{H}\right]
\end{gathered}
$$

holds, where $M_{2}$ does not depend on $\varphi, \psi, f_{s}(1 \leq s \leq N-1)$, or $\tau$.

The second order of accuracy absolutely stable difference schemes was constructed and investigated later than first order difference scheme (175) in $[64,65]$. One second order of accuracy two-step difference scheme generated by CrankNicholson difference scheme

$$
\begin{aligned}
& \tau^{-2}\left(u_{k+1}-2 u_{k}+u_{k-1}\right)+A_{k+1 / 2} 4^{-1}\left(u_{k+1}+u_{k}\right) \\
& +A_{k+1 / 2}^{1 / 2} A_{k-1 / 2}^{1 / 2} 4^{-1}\left(u_{k}+u_{k-1}\right) \\
& +\tau^{-1}\left(A_{k-1 / 2}^{1 / 2}-A_{k+1 / 2}^{1 / 2}\right) A_{k-1 / 2}^{-1 / 2} \tau^{-1}\left(u_{k}-u_{k-1}\right) \\
& +2^{-1} \tau^{-1}\left(A_{k+1}^{1 / 2}-A_{k}^{1 / 2}\right) A_{k+1 / 2}^{-1 / 2} \tau^{-1}\left(u_{k+1}-u_{k}\right) \\
& +A_{k+1 / 2}^{1 / 2} A_{k-1 / 2}^{-1 / 2} 2^{-1} \tau^{-1} \\
& \cdot\left(A_{k}^{1 / 2}-A_{k-1}^{1 / 2}\right) A_{k-1 / 2}^{-1 / 2} \tau^{-1}\left(u_{k}-u_{k-1}\right) \\
& =2^{-1}\left(f_{k-1 / 2}+f_{k+1 / 2}\right) \\
& +2^{-1}\left(A_{k+1 / 2}^{1 / 2}-A_{k-1 / 2}^{1 / 2}\right) A_{k-1 / 2}^{-1 / 2} f_{k-1 / 2}, \\
& A_{k \pm 1 / 2}=A\left(t_{k} \pm \frac{\tau}{2}\right), \quad f_{k \pm 1 / 2}=f\left(t_{k} \pm \frac{\tau}{2}\right), \\
& t_{k}=k \tau, \quad 1 \leq k \leq N-1, \\
& u_{0}=\varphi, \\
& \tau^{-1}\left(u_{1}-u_{0}\right)+\frac{\tau}{2} A_{1 / 2} 2^{-1}\left(u_{1}+u_{0}\right) \\
& +\frac{\tau}{2}\left(A_{1 / 2}^{1 / 2}\right)^{\prime} A_{1 / 2}^{-1 / 2} \tau^{-1}\left(u_{1}-u_{0}\right) \\
& =\frac{\tau}{2} f_{1 / 2}+A_{1 / 2}^{1 / 2} A_{1 / 2}^{-1 / 2} \psi \text {, }
\end{aligned}
$$


for approximately solving problem (174), was presented in [64]. The following stability estimates of the solution of the difference method and its first and second order difference derivatives were established. Let us have the above estimates.

Theorem 84 (see [64]). Assume that all assumptions of Theorem 82 are satisfied. Then, for the solution of difference scheme (180), the following stability estimate

$$
\begin{aligned}
& \max _{1 \leq k \leq N}\left\|\frac{u_{k}-u_{k-1}}{\tau}\right\|_{H}+\max _{0 \leq k \leq N}\left\|u_{k}\right\|_{H} \\
& \quad \leq M_{2}\left[\left\|A^{1 / 2}(0) \varphi\right\|_{H}+\|\psi\|_{H}+\sum_{s=1}^{N-1}\left\|f_{s+1 / 2}\right\|_{H} \tau\right]
\end{aligned}
$$

holds, where $M_{2}$ does not depend on $\varphi, \psi, f_{s+1 / 2}(0 \leq s \leq N-$ 1), or $\tau$.

Theorem 85 (see [64]). Assume that all assumptions of Theorem 83 are satisfied. Then, for the solution of difference scheme (180), the following stability estimate

$$
\begin{gathered}
\max _{1 \leq k \leq N}\left\|A^{1 / 2}(0) \frac{u_{k}-u_{k-1}}{\tau}\right\|_{H} \\
\max _{1 \leq k \leq N-1} \| A_{k+1 / 2} 4^{-1}\left(u_{k+1}+u_{k}\right) \\
+A_{k+1 / 2}^{1 / 2} A_{k-1 / 2}^{1 / 2} 4^{-1}\left(u_{k}+u_{k-1}\right) \\
+\tau^{-1}\left(A_{k-1 / 2}^{1 / 2}-A_{k+1 / 2}^{1 / 2}\right) A_{k-1 / 2}^{-1 / 2} \tau^{-1}\left(u_{k}-u_{k-1}\right) \\
+2^{-1} \tau^{-1}\left(A_{k+1}^{1 / 2}-A_{k}^{1 / 2}\right) A_{k+1 / 2}^{-1 / 2} \tau^{-1}\left(u_{k+1}-u_{k}\right) \\
+A_{k+1 / 2}^{1 / 2} A_{k-1 / 2}^{-1 / 2} 2^{-1} \tau^{-1}\left(A_{k}^{1 / 2}-A_{k-1}^{1 / 2}\right) \\
\cdot A_{k-1 / 2}^{-1 / 2} \tau^{-1}\left(u_{k}-u_{k-1}\right) \|_{H}, \\
+\max _{1 \leq k \leq N-1}\left\|\tau^{-2}\left(u_{k+1}-2 u_{k}+u_{k-1}\right)\right\|_{H} \\
+M_{2}\left[\left\|\sum_{s=1}^{N-1}\right\| f_{s+1 / 2}-f_{s-1 / 2} \|_{H}\right]
\end{gathered}
$$

holds, where $M_{2}$ does not depend on $\varphi, \psi, f_{s+1 / 2}(0 \leq s \leq$ $N-1)$, or $\tau$.
Another second order of accuracy two-step difference scheme generated by the second order of accuracy implicit difference scheme

$$
\begin{aligned}
& \tau^{-2}\left(u_{k+1}-2 u_{k}+u_{k-1}\right) \\
& =\left[A_{k+1}^{1 / 2}+\frac{\tau}{2}\left(A_{k+1}^{1 / 2}\right)^{\prime}\right] \\
& \cdot\left\{-A_{k+1}^{1 / 2} u_{k+1}\right. \\
& +\left[\frac{\tau}{2} A_{k+1}-\frac{1}{2} A_{k+1}^{-1 / 2}\left(A_{k+1}^{1 / 2}\right)^{\prime}\right] \\
& \cdot A_{k+3 / 2}^{-1 / 2}\left[\tau^{-1}\left(u_{k+1}-u_{k}\right)\right. \\
& \left.-\frac{\tau}{2} A_{k+1} u_{k+1}+\frac{\tau}{2} f_{k+1}\right] \\
& \left.+A_{k+1}^{-1 / 2} f_{k+1}\right\} \\
& -\left\{\left[A_{k+1}^{1 / 2}+\frac{\tau}{2}\left(A_{k+1}^{1 / 2}\right)^{\prime}\right] A_{k+1}^{-1 / 2}\left(A_{k+1}^{1 / 2}\right)^{\prime} A_{k+1 / 2}^{-1 / 2}\right. \\
& \left.-\tau^{-1}\left[\left(A_{k+1}^{1 / 2}-A_{k}^{1 / 2}\right)+\frac{\tau}{2}\left(\left(A_{k+1}^{1 / 2}\right)^{\prime}-\left(A_{k}^{1 / 2}\right)^{\prime}\right)\right]\right\} \\
& \cdot A_{k+1 / 2}^{-1 / 2} \times\left[\tau^{-1}\left(u_{k}-u_{k-1}\right)-\frac{\tau}{2} A_{k} u_{k}+\frac{\tau}{2} f_{k}\right] \\
& +2^{-1}\left(A_{k+1} u_{k+1}-A_{k} u_{k}\right)-2^{-1}\left(f_{k+1}-f_{k}\right) \text {, } \\
& A_{k}=A\left(t_{k}\right), \quad A_{k \pm 1 / 2}=A\left(t_{k} \pm \frac{\tau}{2}\right), \quad f_{k}=f\left(t_{k}\right), \\
& t_{k}=k \tau, \quad 1 \leq k \leq N-1 \text {, } \\
& u_{0}=\varphi \text {, } \\
& \tau^{-1}\left(u_{1}-u_{0}\right)+\frac{\tau}{2} A_{1 / 2} 2^{-1}\left(u_{1}+u_{0}\right) \\
& +\frac{\tau}{2}\left(A_{1 / 2}^{1 / 2}\right)^{\prime} A_{1 / 2}^{-1 / 2} \tau^{-1}\left(u_{1}-u_{0}\right) \\
& =\frac{\tau}{2} f_{1}+A_{1 / 2}^{1 / 2} A_{1 / 2}^{-1 / 2} \psi
\end{aligned}
$$

for approximately solving problem (174) was constructed in [65]. Similar stability estimates of the solution of difference scheme (180) and its first and second order difference derivatives were established under the same conditions.

However, difference schemes (180) and (183) were generated by the square root of $A(t)$. Thus, for a practical realization of these difference methods, it is necessary to first construct operator $A^{1 / 2}(t)$, which obviously is not easy. Hence, in spite of theoretical results, the application of these methods for numerically solving an initial-value problem is not very practical. It is important to study the difference schemes generated by integer powers of $A(t)$. 
In $[68,69]$, a second order of accuracy of two types of difference schemes

$$
\begin{gathered}
\frac{u_{k+1}-2 u_{k}+u_{k-1}}{\tau^{2}}+\frac{1}{2} A_{k} u_{k} \\
+\frac{1}{4} A_{k}\left(u_{k+1}+u_{k-1}\right)=f_{k}, \\
A_{k}=A\left(t_{k}\right), \quad f_{k}=f\left(t_{k}\right), \\
t_{k}=k \tau, \quad 1 \leq k \leq N-1, N \tau=T, \\
\left(I+\tau^{2} A_{0}\right) \tau^{-1}\left(u_{1}-u_{0}\right)=\frac{\tau}{2}\left(f_{0}-A_{0} u_{0}\right)+\psi, \\
f_{0}=f(0), \quad u_{0}=\varphi, \\
\frac{u_{k+1}-2 u_{k}+u_{k-1}+A_{k} u_{k}+\frac{\tau^{2}}{4} A_{k}^{2} u_{k+1}=f_{k},}{\tau^{2}} \\
A_{k}=A\left(t_{k}\right), \quad f_{k}=f\left(t_{k}\right), \\
t_{k}=k \tau, \quad 1 \leq k \leq N-1, N \tau=T, \\
\left(I+\tau^{2} A_{0}\right) \tau^{-1}\left(u_{1}-u_{0}\right)=\frac{\tau}{2}\left(f_{0}-A_{0} u_{0}\right)+\psi, \\
f_{0}=f(0), \quad u_{0}=\varphi
\end{gathered}
$$

generated by integer powers of $A(t)$ for approximately solving problem (174) was presented. The stability estimates for the solution of these difference schemes and for the first and second order difference derivatives were established. The theoretical statements for this difference method were supported by the numerical experiments for one-dimensional hyperbolic partial differential equation with the Dirichlet boundary condition.

7.3. Nonlocal Problems. In [120], the nonlocal boundary value problem for hyperbolic equations

$$
\begin{gathered}
\frac{d^{2} v(t)}{d t^{2}}+A v(t)=f(t) \quad(0 \leq t \leq T), \\
v(0)=\alpha v(T)+\varphi, \quad v^{\prime}(0)=\beta v^{\prime}(T)+\psi
\end{gathered}
$$

was considered.

Function $v(t)$ is called a solution of problem (186) if the following conditions are satisfied:

(i) $v(t)$ is twice continuously differentiable on the interval $[0, T]$ and continuously differentiable on the segment $[0, T]$.

(ii) The element $v(t)$ belongs to $D(A)$ for all $t \in[0, T]$, and the function $A v(t)$ is continuous on the segment $[0, T]$.

(iii) $v(t)$ satisfies the equation and nonlocal boundary conditions (186).

Theorem 86 (see [120]). Suppose that $\varphi \in D(A), \psi \in$ $D\left(A^{1 / 2}\right)$, and $f(t)$ are continuously differentiable on $[0, T]$ function and $|1+\alpha \beta|>|\alpha+\beta|$. Then there is a unique solution of problem (186) and the stability inequalities

$$
\begin{aligned}
& \max _{0 \leq t \leq T}\|v(t)\|_{H} \\
& \leq M\left[\|\varphi\|_{H}+\left\|A^{-1 / 2} \psi\right\|_{H}+\max _{0 \leq t \leq T}\left\|A^{-1 / 2} f(t)\right\|_{H}\right], \\
& \max _{0 \leq t \leq T}\left\|A^{1 / 2} v(t)\right\|_{H} \\
& \leq M\left[\left\|A^{1 / 2} \varphi\right\|_{H}+\|\psi\|_{H}+\max _{0 \leq t \leq T}\|f(t)\|_{H}\right], \\
& \max _{0 \leq t \leq T}\left\|\frac{d^{2} v(t)}{d t^{2}}\right\|_{H}+\max _{0 \leq t \leq T}\|A v(t)\|_{H} \\
& \leq M\left[\|A \varphi\|_{H}+\left\|A^{1 / 2} \psi\right\|_{H}+\|f(0)\|_{H}+\int_{0}^{T}\left\|f^{\prime}(t)\right\|_{H} d t\right]
\end{aligned}
$$

hold, where $M$ does not depend on $f(t), t \in[0, T]$, or $\varphi, \psi$.

Two applications of Theorem 86 were presented. First, the mixed boundary value problem for hyperbolic equations

$$
\begin{gathered}
u_{t t}-\left(a(x) u_{x}\right)_{x}+u=f(t, x), \\
0<t<T, \quad 0<x<1, \\
u(0, x)=\alpha u(T, x)+\varphi(x), \\
u_{t}(0, x)=\beta u_{t}(T, x)+\psi(x), \\
0 \leq x \leq 1, \\
u(t, 0)=u(t, 1), \\
u_{x}(t, 0)=u_{x}(t, 1), \\
0 \leq t \leq T,
\end{gathered}
$$

was considered. Problem (188) has unique smooth solution $u(t, x)$ for $|1+\alpha \beta|>|\alpha|+|\beta|$ and the smooth $a(x) \geq$ $a>0(x \in(0,1)), a(1)=a(0), \varphi(x), \psi(x)(x \in[0,1])$, and $f(t, x)(t \in[0, T], x \in[0,1])$ functions. This allows us to reduce mixed problem (188) to nonlocal boundary value problem (186) in Hilbert space $H$ with self-adjoint positive definite operator $A$ defined by (188).

Theorem 87 (see [120]). For solutions of mixed problem (188), the stability inequalities

$$
\begin{aligned}
& \max _{0 \leq t \leq T}\|u\|_{W_{2}^{1}[0,1]} \\
& \leq M\left[\max _{0 \leq t \leq T}\|f\|_{L_{2}[0,1]}+\|\varphi\|_{W_{2}^{1}[0,1]}+\|\psi\|_{L_{2}[0,1]}\right] \\
& \max _{0 \leq t \leq T}\|u\|_{W_{2}^{2}[0,1]}+\max _{0 \leq t \leq T}\left\|u_{t t}\right\|_{L_{2}[0,1]} \\
& \leq M\left[\max _{0 \leq t \leq T}\left\|f_{t}\right\|_{L_{2}[0,1]}+\|f(0)\|_{L_{2}[0,1]}\right. \\
& \left.\quad+\|\varphi\|_{W_{2}^{2}[0,1]}+\|\psi\|_{W_{2}^{1}[0,1]}\right]
\end{aligned}
$$

hold, where $M$ does not depend on $f(t, x)$ or $\varphi(x), \psi(x)$. 
Second, let $\Omega$ be the unit open cube in the $n$-dimensional Euclidean space $\mathbb{R}^{n}\left(0<x_{k}<1,1 \leq k \leq n\right)$ with boundary $S, \bar{\Omega}=\Omega \cup S$. In $[0, T] \times \Omega$, the mixed boundary value problem for the multidimensional hyperbolic equation

$$
\begin{gathered}
\frac{\partial^{2} u(t, x)}{\partial t^{2}}-\sum_{r=1}^{n}\left(a_{r}(x) u_{x_{r}}\right)_{x_{r}}=f(t, x), \\
x=\left(x_{1}, \ldots, x_{n}\right) \in \Omega, \quad 0<t<T, \\
u(0, x)=\alpha u(T, x)+\varphi(x), \\
\frac{\partial u(0, x)}{\partial t}=\beta \frac{\partial u(T, x)}{\partial t}+\psi(x), \\
x \in \bar{\Omega}, \\
u(t, x)=0, \quad x \in S,
\end{gathered}
$$

was considered, where $a_{r}(x),(x \in \Omega), \varphi(x), \psi(x)(x \in \bar{\Omega})$ and $f(t, x)(t \in(0, T), x \in \Omega)$ are given smooth functions and $a_{r}(x) \geq 0$.

Problem (190) has unique smooth solution $u(t, x)$ for $|1+\alpha \beta|>|\alpha|+|\beta|$ and the smooth $a_{r}(x) \geq 0$ and $f(t, x)$ functions. This allows us to reduce mixed problem (190) to nonlocal boundary value problem (186) in Hilbert space $H$ with self-adjoint positive definite operator $A$ defined by (190).

Theorem 88 (see [120]). For solutions of mixed problem (190), the stability inequalities

$$
\begin{gathered}
\max _{0 \leq t \leq T}\|u\|_{W_{2}^{1}(\bar{\Omega})} \\
\leq M\left[\max _{0 \leq t \leq T}\|f\|_{L_{2}(\bar{\Omega})}+\|\varphi\|_{W_{2}^{1}(\bar{\Omega})}+\|\psi\|_{L_{2}(\bar{\Omega})}\right] \\
\max _{0 \leq t \leq T}\|u\|_{W_{2}^{2}(\bar{\Omega})}+\max _{0 \leq t \leq T}\left\|u_{t t}\right\|_{L_{2}(\bar{\Omega})} \\
\leq M\left[\max _{0 \leq t \leq T}\left\|f_{t}\right\|_{L_{2}(\bar{\Omega})}+\|f(0)\|_{L_{2}(\bar{\Omega})}\right. \\
\left.\quad\|\varphi\|_{W_{2}^{2}(\bar{\Omega})}+\|\psi\|_{W_{2}^{1}(\bar{\Omega})}\right]
\end{gathered}
$$

hold, where $M$ does not depend on $f(t, x)$ or $\varphi(x), \psi(x)$.

Futhermore, the first order of accuracy difference scheme

$$
\begin{gathered}
\tau^{-2}\left(u_{k+1}-2 u_{k}+u_{k-1}\right)+A u_{k+1}=f_{k}, \\
f_{k}=f\left(t_{k+1}\right), \\
t_{k+1}=(k+1) \tau, \quad 1 \leq k \leq N-1, \\
u_{0}=\alpha u_{N}+\varphi, \\
\tau^{-1}\left(u_{1}-u_{0}\right)=\beta \tau^{-1}\left(u_{N}-u_{N-1}\right)+\psi
\end{gathered}
$$

for approximately solving boundary value problem (186) was considered.

The stability of solutions of difference scheme (192) was investigated under the assumption

$$
|1+\alpha \beta|>|\alpha+\beta| \text {. }
$$

Theorem 89 (see [120]). Let $\varphi \in D(A), \psi \in D\left(A^{1 / 2}\right)$, and $1>|\alpha||\beta|+|\alpha|+|\beta|$. Then, for the solution of difference scheme (192), the stability inequalities

$$
\begin{gathered}
\left\|u_{k}\right\|_{H} \leq M\left\{\sum_{s=1}^{N-1}\left\|A^{-1 / 2} f_{s}\right\|_{H} \tau\right. \\
\left.+A^{-1 / 2}\|\psi\|_{H}+\|\varphi\|_{H}\right\}, \quad k=0,2, \ldots, N, \\
\left\|u_{1}\right\|_{H} \leq M\left[\|\varphi\|_{H}+\left\|\left(I+i \tau A^{1 / 2}\right) A^{-1 / 2} \psi\right\|_{H}\right], \\
\left\|A^{1 / 2} u_{k}\right\|_{H} \leq M\left\{\sum_{s=1}^{N-1}\left\|f_{s}\right\|_{H} \tau+\|\psi\|_{H}\right. \\
\left.+\left\|A^{1 / 2} \varphi\right\|_{H}\right\}, \quad k=0,2, \ldots, N, \\
\left\|A^{1 / 2} u_{1}\right\|_{H} \leq M\left[\left\|A^{1 / 2} \varphi\right\|_{H}+\left\|\left(I+i \tau A^{1 / 2}\right) \psi\right\|_{H}\right], \\
\left\|A u_{k}\right\|_{H} \leq M\left\{\sum_{s=2}^{N-1}\left\|f_{s}-f_{s-1}\right\|_{H}+\left\|f_{1}\right\|_{H}\right. \\
\left.+\left\|A^{1 / 2} \psi\right\|_{H}+\|A \varphi\|_{H}\right\}, \\
\left\|A u_{1}\right\|_{H} \leq M\left[\|A \varphi\|_{H}+\left\|\left(I+i \tau A^{1 / 2}\right) A^{1 / 2} \psi\right\|_{H}\right]
\end{gathered}
$$

hold, where $M$ does not depend on $f_{s}, 1 \leq s \leq N-1$, or $\varphi$, $\psi$.

Note that these stability estimates in the case $k=1$ are weaker than the respective estimates in the cases $k=$ $0,2, \ldots, N$. However, obtaining this type of estimate is important for applications. Consider $a^{\tau}=\left(a_{k}\right)$ denotes the mesh function of the approximation. And $\left\|\left(I+i \tau A^{-1 / 2}\right) a_{1}\right\|_{H} \sim$ $\left\|a_{1}\right\|_{H}=o(\tau)$ assume that $\tau\left\|A a_{1}\right\|_{H}$ tends to 0 as $\tau \rightarrow$ 0 not slower than $\left\|a_{1}\right\|_{H}$. It takes place in applications by supplementary restriction of the smoothness property of the data in the space variables. It is clear that the uniformity in $\tau$ estimate

$$
\left\|u_{1}\right\|_{H} \leq\|\varphi\|_{H}+\left\|A^{-1 / 2} \psi\right\|_{H}
$$

is absent. However, estimates for the solution of first order of accuracy modified difference scheme for approximately solving boundary value problem (186)

$$
\begin{gathered}
\tau^{-2}\left(u_{k+1}-2 u_{k}+u_{k-1}\right)+A u_{k+1}=f_{k}, \\
f_{k}=f\left(t_{k}\right), \\
t_{k}=k \tau, \quad 1 \leq k \leq N-1, \\
u_{0}=\alpha u_{N}+\varphi, \\
\left(I+\tau^{2} A\right) \tau^{-1}\left(u_{1}-u_{0}\right)=\beta \tau^{-1}\left(u_{N}-u_{N-1}\right)+\psi
\end{gathered}
$$

are better than the estimates for the solution of difference scheme (192). 
Theorem 90 (see [120]). Let $\varphi \in D(A), \psi \in D\left(A^{1 / 2}\right)$, and $1>|\alpha||\beta|+|\alpha|+|\beta|$. Then for the solution of difference scheme (196) the stability inequalities

$$
\begin{aligned}
& \max _{0 \leq k \leq N}\left\|u_{k}\right\|_{H} \leq M\left\{\sum_{s=1}^{N-1}\left\|A^{-1 / 2} f_{s}\right\|_{H} \tau+\left\|A^{-1 / 2} \psi\right\|_{H}+\|\varphi\|_{H}\right\}, \\
& \max _{0 \leq k \leq N}\left\|A^{1 / 2} u_{k}\right\|_{H} \leq M\left\{\sum_{s=1}^{N-1}\left\|f_{s}\right\|_{H} \tau+\left\|A^{1 / 2} \varphi\right\|_{H}+\|\psi\|_{H}\right\}, \\
& \max _{1 \leq k \leq N-1}\left\|\tau^{-2}\left(u_{k+1}-2 u_{k}+u_{k-1}\right)\right\|_{H}+\max _{0 \leq k \leq N}\left\|A u_{k}\right\|_{H} \\
& \leq M\left\{\sum_{s=2}^{N-1}\left\|f_{s}-f_{s-1}\right\|_{H}+\left\|f_{1}\right\|_{H}+\left\|A^{1 / 2} \psi\right\|_{H}+\|A \varphi\|_{H}\right\}
\end{aligned}
$$

hold, where $M$ does not depend on $f_{s}, 1 \leq s \leq N-1$, or $\varphi, \psi$.

Moreover, two types of the second order of accuracy difference schemes

$$
\begin{gathered}
\tau^{-2}\left(u_{k+1}-2 u_{k}+u_{k-1}\right)+A u_{k}+\frac{\tau^{2}}{4} A^{2} u_{k+1}=f_{k}, \\
f_{k}=f\left(t_{k}\right), \\
t_{k}=k \tau, \quad 1 \leq k \leq N-1, \\
\left(I+\frac{\tau^{2} A}{2}\right) \tau^{-1}\left(u_{1}-u_{0}\right)-\frac{\tau}{2}\left(f_{0}-A u_{0}\right) \\
=\beta\left[\tau^{-1}\left(u_{N}-u_{N-1}\right)+\frac{\tau}{2}\left(f_{N}-A u_{N}\right)\right]+\psi, \\
f_{0}=f(0), \quad u_{N}=f(1), \alpha u_{N}+\varphi, \\
\tau^{-2}\left(u_{k+1}-2 u_{k}+u_{k-1}\right)+\frac{1}{2} A u_{k}+\frac{1}{4} A\left(u_{k+1}+u_{k-1}\right)=f_{k}, \\
f_{k}=f\left(t_{k}\right), \\
t_{k}=k \tau, \quad 1 \leq k \leq N-1, \\
\left(I+\frac{\tau^{2} A}{4}\right)\left[\left(I+\frac{\tau^{2} A}{4}\right) \tau^{-1}\left(u_{1}-u_{0}\right)-\frac{\tau}{2}\left(f_{0}-A u_{0}\right)\right] \\
=\beta\left[\tau^{-1}\left(u_{N}-u_{N-1}\right)+\frac{\tau}{2}\left(f_{N}-A u_{N}\right)\right]+\psi, \\
f_{0}=f(0), \quad f_{N}=f(1), \\
u_{0}=\alpha u_{N}+\varphi
\end{gathered}
$$

$$
\begin{gathered}
\max _{0 \leq k \leq N}\left\|u_{k}\right\|_{H} \leq M\left\{\sum_{s=0}^{N}\left\|A^{-1 / 2} f_{s}\right\|_{H} \tau+\left\|A^{-1 / 2} \psi\right\|_{H}+\|\varphi\|_{H}\right\}, \\
\max _{0 \leq k \leq N}\left\|A^{1 / 2} u_{k}\right\|_{H} \leq M\left\{\sum_{s=0}^{N}\left\|f_{s}\right\|_{H} \tau+\left\|A^{1 / 2} \varphi\right\|_{H}+\|\psi\|_{H}\right\}, \\
\max _{1 \leq k \leq N-1}\left\|\tau^{-2}\left(u_{k+1}-2 u_{k}+u_{k-1}\right)\right\|_{H}+\max _{0 \leq k \leq N}\left\|A u_{k}\right\|_{H} \\
\leq M\left\{\sum_{s=1}^{N}\left\|f_{s}-f_{s-1}\right\|_{H}+\left\|f_{0}\right\|_{H}+\left\|A^{1 / 2} \psi\right\|_{H}+\|A \varphi\|_{H}\right\}
\end{gathered}
$$

hold, where $M$ does not depend on $f_{s}, 0 \leq s \leq N$, or $\varphi, \psi$.

Note that one can construct the difference schemes of the second order of approximation over time and of an arbitrary order of approximation over space variables for approximate solutions of boundary value problems (188) and (190). Abstract Theorems 91 and 92 permit us to obtain stability estimates for the solutions of these difference schemes. 
In $[73,75]$, a third order of accuracy difference scheme

$$
\begin{aligned}
& \tau^{-2}\left(u_{k+1}-2 u_{k}+u_{k-1}\right)+\frac{2}{3} A u_{k} \\
& +\frac{1}{6} A\left(u_{k+1}+u_{k-1}\right)+\frac{1}{12} \tau^{2} A^{2} u_{k+1}=f_{k} \text {, } \\
& f_{k}=\frac{2}{3} f\left(t_{k}\right)+\frac{1}{6}\left(f\left(t_{k+1}\right)+f\left(t_{k-1}\right)\right) \\
& -\frac{1}{12} \tau^{2}\left(-A f\left(t_{k+1}\right)+f^{\prime \prime}\left(t_{k+1}\right)\right), \\
& t_{k}=k \tau, \quad 1 \leq k \leq N-1, \\
& u_{0}=\alpha u_{N}+\varphi, \\
& \left(I+\frac{\tau^{2}}{12} A+\frac{\tau^{4}}{144} A^{2}\right) \tau^{-1}\left(u_{1}-u_{0}\right)+\frac{\tau}{2} A u_{0}-\tau f_{1,1} \\
& =\beta\left(I-\frac{\tau^{2} A}{12}\right)\left(\frac{7 u_{N}-8 u_{N-1}+u_{N-2}}{6 \tau}+\frac{\tau}{3}\left(f_{N}-A u_{N}\right)\right) \\
& +\left(I-\frac{\tau^{2} A}{12}\right) \psi \\
& f_{1,1}=\frac{1}{2} f(0)+f^{\prime}(0) \frac{\tau}{6}
\end{aligned}
$$

and a fourth order of accuracy difference scheme

$$
\begin{gathered}
\tau^{-2}\left(u_{k+1}-2 u_{k}+u_{k-1}\right)+\frac{5}{6} A u_{k} \\
+\frac{1}{12} A\left(u_{k+1}+u_{k-1}\right)-\frac{\tau^{2}}{72} A^{2} u_{k} \\
+\frac{\tau^{2}}{144} A^{2}\left(u_{k+1}+u_{k-1}\right)=f_{k}, \\
f_{k}=\frac{5}{6} f\left(t_{k}\right)+\frac{1}{12}\left(f\left(t_{k+1}\right)+f\left(t_{k-1}\right)\right) \\
+\frac{\tau^{2}}{72}\left(-A f\left(t_{k}\right)+f^{\prime \prime}\left(t_{k}\right)\right) \\
-\frac{1}{144} \tau^{2}\left(-A\left(f\left(t_{k+1}\right)+f\left(t_{k-1}\right)\right)\right. \\
\left.\quad+f^{\prime \prime}\left(t_{k+1}\right)+f^{\prime \prime}\left(t_{k-1}\right)\right), \\
t_{k}=k \tau, \quad 1 \leq k \leq N-1,
\end{gathered}
$$

$$
\begin{aligned}
& u_{0}=\alpha u_{N}+\varphi, \\
& \left(I+\frac{\tau^{2}}{12} A+\frac{\tau^{4}}{144} A^{2}\right) \tau^{-1}\left(u_{1}-u_{0}\right)+\frac{\tau}{2} A u_{0}-\tau f_{2,2} \\
& =\beta\left(I-\frac{\tau^{2} A}{12}\right)\left(\frac{85 u_{N}-108 u_{N-1}+27 u_{N-2}-4 u_{N-3}}{66 \tau}\right. \\
& \left.+\frac{3 \tau}{11}\left(f_{N}-A u_{N}\right)\right) \\
& +\left(I-\frac{\tau^{2} A}{12}\right) \psi \\
& f_{2,2}=\left\{\left(I-\frac{\tau^{2} A}{12}\right) f(0)\right. \\
& +\left(-\left(I-\frac{5 \tau^{2} A}{12}\right) f(0)+\tau f^{\prime}(0)\right) \frac{1}{2} \\
& +\left(-A \tau f(0)-2 f^{\prime}(0)+\tau f^{\prime \prime}(0)\right) \frac{\tau}{6} \\
& \left.+\left(A f(0)-3 f^{\prime \prime}(0)\right) \frac{\tau^{2}}{24}\right\}
\end{aligned}
$$

for approximately solving nonlocal boundary value problem (186) were constructed.

Theorem 93 (see $[73,75]$ ). Suppose that the assumption

$$
|\alpha|+2|\beta|+2|\alpha||\beta|<1
$$

is satisfied and $\varphi \in D\left(A^{3 / 2}\right), \psi \in D\left(A^{1 / 2}\right)$. Then, for the solution of difference scheme (202), the following stability estimates hold:

$$
\begin{gathered}
\max _{0 \leq k \leq N}\left\|u_{k}\right\|_{H} \\
\leq M\left\{\sum_{s=1}^{N-1}\left\|A^{-1 / 2} f_{s}\right\|_{H} \tau+\left\|\left(I+i \tau A^{1 / 2}\right) \varphi\right\|_{H}\right. \\
\left.+\left\|A^{-1 / 2} \psi\right\|_{H}+\tau\left\|A^{-1 / 2} f_{1,1}\right\|_{H}\right\}, \\
\max _{0 \leq k \leq N}\left\|A^{1 / 2} u_{k}\right\|_{H} \\
\leq M\left\{\sum_{s=1}^{N-1}\left\|f_{s}\right\|_{H} \tau+\left\|A^{1 / 2}\left(I+i \tau A^{1 / 2}\right) \varphi\right\|_{H}\right. \\
\left.+\|\psi\|_{H}+\tau\left\|f_{1,1}\right\|_{H}\right\},
\end{gathered}
$$




$$
\begin{aligned}
\max _{0 \leq k \leq N}\left\|A u_{k}\right\|_{H} & \\
\leq M\{ & \left\{\sum_{s=2}^{N-1}\left\|f_{s}-f_{s-1}\right\|_{H}+\left\|f_{1}\right\|_{H}+\left\|A\left(I+i \tau A^{1 / 2}\right) \varphi\right\|_{H}\right. \\
& \left.+\left\|A^{1 / 2} \psi\right\|_{H}+\tau\left\|A^{1 / 2} f_{1,1}\right\|_{H}\right\},
\end{aligned}
$$

where $M$ does not depend on $\tau, \varphi, \psi, f_{1,1}$, or $f_{s}, 1 \leq s \leq N-1$.

Theorem 94 (see $[73,75]$ ). Suppose that assumption (204) holds and $\varphi \in D\left(A^{1 / 2}\right), \psi \in D\left(A^{1 / 2}\right)$. Then, for the solution of difference scheme (203), the following stability estimates hold:

$$
\begin{aligned}
& \max _{0 \leq k \leq N}\left\|u_{k}\right\|_{H} \leq M\left\{\sum_{s=1}^{N-1}\left\|A^{-1 / 2} f_{s}\right\|_{H} \tau+\|\varphi\|_{H}\right. \\
& \left.+\left\|A^{-1 / 2} \psi\right\|_{H}+\tau\left\|A^{-1 / 2} f_{2,2}\right\|_{H}\right\}, \\
& \max _{0 \leq k \leq N}\left\|A^{1 / 2} u_{k}\right\|_{H} \\
& \leq M\left\{\sum_{s=1}^{N-1}\left\|f_{s}\right\|_{H} \tau+\left\|A^{1 / 2} \varphi\right\|_{H}+\|\psi\|_{H}+\tau\left\|f_{2,2}\right\|_{H}\right\}, \\
& \max _{0 \leq k \leq N}\left\|A u_{k}\right\|_{H} \\
& \leq M\left\{\sum_{s=2}^{N-1}\left\|f_{s}-f_{s-1}\right\|_{H}+\left\|f_{1}\right\|_{H}+\|A \varphi\|_{H}\right. \\
& \left.+\left\|A^{1 / 2} \psi\right\|_{H}+\tau\left\|A^{1 / 2} f_{2,2}\right\|_{H}\right\},
\end{aligned}
$$

where $M$ does not depend on $\tau, \varphi, \psi, f_{2,2}$, or $f_{s}, 1 \leq s \leq N-1$.

In [72], the multipoint nonlocal boundary value problem

$$
\begin{gathered}
\frac{d^{2} v(t)}{d t^{2}}+A v(t)=f(t) \quad(0 \leq t \leq T), \\
v(0)=\sum_{j=1}^{n} \alpha_{j} v\left(\lambda_{j}\right)+\varphi \\
v_{t}(0)=\sum_{j=1}^{n} \beta_{j} v_{t}\left(\lambda_{j}\right)+\psi \\
0<\lambda_{1} \leq \lambda_{2} \leq \cdots \leq \lambda_{n} \leq T
\end{gathered}
$$

for a differential equation in Hilbert space $H$ with the selfadjoint positive definite operator $A$ was considered.

Function $v(t)$ is called a solution of problem (207) if the following conditions are satisfied:

(i) $v(t)$ is twice continuously differentiable on the interval $[0, T]$ and continuously differentiable on the segment $[0, T]$. (ii) The element $v(t)$ belongs to $D(A)$ for all $t \in[0, T]$, and the function $A v(t)$ is continuous on the segment $[0, T]$.

(iii) $v(t)$ satisfies the equation and nonlocal boundary conditions (207).

Theorem 95 (see [72]). Suppose that $\varphi \in D(A), \psi \in D\left(A^{1 / 2}\right)$, $f(t)$ is continuously differentiable on $[0, T]$ function and the assumption

$$
\sum_{k=1}^{n}\left|\alpha_{k}+\beta_{k}\right|+\sum_{k=1}^{n}\left|\alpha_{k}\right| \sum_{m=1}^{n}\left|\beta_{m}\right|<\left|1+\sum_{k=1}^{n} \alpha_{k} \beta_{k}\right|
$$

holds. Then there is a unique solution of problem (207) and the stability inequalities

$$
\begin{aligned}
& \max _{0 \leq t \leq T}\|v(t)\|_{H} \\
& \quad \leq M\left[\|\varphi\|_{H}+\left\|A^{-1 / 2} \psi\right\|_{H}+\max _{0 \leq t \leq T}\left\|A^{-1 / 2} f(t)\right\|_{H}\right], \\
& \max _{0 \leq t \leq T}\left\|A^{1 / 2} v(t)\right\|_{H} \\
& \quad \leq M\left[\left\|A^{1 / 2} \varphi\right\|_{H}+\|\psi\|_{H}+\max _{0 \leq t \leq T}\|f(t)\|_{H}\right], \\
& \max _{0 \leq t \leq T}\left\|\frac{d^{2} v(t)}{d t^{2}}\right\|_{H}+\max _{0 \leq t \leq T}\|A v(t)\|_{H} \\
& \quad \leq M\left[\|A \varphi\|_{H}+\left\|A^{1 / 2} \psi\right\|_{H}+\|f(0)\|_{H}+\int_{0}^{T}\left\|f^{\prime}(t)\right\|_{H} d t\right]
\end{aligned}
$$

hold, where $M$ does not depend on $\varphi, \psi$, or $f(t), t \in[0,1]$.

In practice, the mixed multipoint nonlocal boundary value problem

$$
\begin{gathered}
u_{t t}-\left(a(x) u_{x}\right)_{x}+\delta u=f(t, x), \\
0<t<T, \quad 0<x<1, \\
u(0, x)=\sum_{j=1}^{n} \alpha_{j} u\left(\lambda_{j}, x\right)+\varphi(x), \\
u_{t}(0, x)=\sum_{k=1}^{n} \beta_{k} u_{t}\left(\lambda_{k}, x\right)+\psi(x), \\
u(t, 0)=u(t, 1), \\
u_{x}(t, 0)=u_{x}(t, 1), \\
0 \leq t \leq T,
\end{gathered}
$$


for one-dimensional hyperbolic equations with nonlocal boundary conditions and the nonlocal boundary value problem

$$
\begin{gathered}
\frac{\partial^{2} u(t, x)}{\partial t^{2}}-\sum_{r=1}^{n}\left(a_{r}(x) u_{x_{r}}\right)_{x_{r}}=f(t, x), \\
x=\left(x_{1}, \ldots, x_{n}\right) \in \Omega, \quad 0<t<T, \\
u(0, x)=\sum_{j=1}^{n} \alpha_{j} u\left(\lambda_{j}, x\right)+\varphi(x), \quad x \in \bar{\Omega}, \\
u_{t}(0, x)=\sum_{k=1}^{n} \beta_{k} u_{t}\left(\lambda_{k}, x\right)+\psi(x), \quad x \in \bar{\Omega}, \\
\quad u(t, x)=0, \quad x \in S ; \\
0<\lambda_{1}<\lambda_{2}<\cdots<\lambda_{n} \leq T,
\end{gathered}
$$

for the multidimensional hyperbolic equation with Dirichlet condition were considered. The stability estimates for solution of these problems were established.

We associate multipoint boundary value problem (207) with the corresponding first order of accuracy difference scheme

$$
\begin{gathered}
\tau^{-2}\left(u_{k+1}-2 u_{k}+u_{k-1}\right)+A u_{k+1}=f_{k}, \\
f_{k}=f\left(t_{k}\right), \\
t_{k}=k \tau, \quad 1 \leq k \leq N-1, \\
u_{0}=\sum_{r=1}^{n} \alpha_{r} u_{\left[\lambda_{r} / \tau\right]}+\varphi, \\
\tau^{-1}\left(u_{1}-u_{0}\right)=\sum_{r=1}^{n} \beta_{r}\left(u_{\left[\lambda_{r} / \tau\right]+1}-u_{\left[\lambda_{r} / \tau\right]}\right) \frac{1}{\tau}+\psi .
\end{gathered}
$$

Theorem 96 (see [72]). Suppose that $\varphi \in D(A), \psi \in D\left(A^{1 / 2}\right)$, and the assumption

$$
\sum_{k=1}^{n}\left|\alpha_{k}\right|+\sum_{k=1}^{n}\left|\beta_{k}\right|+\sum_{k=1}^{n}\left|\alpha_{k}\right| \sum_{k=1}^{n}\left|\beta_{k}\right|<1
$$

is satisfied. Then, for the solution of difference scheme (212), the following stability estimates

$$
\begin{gathered}
\left\|u_{k}\right\|_{H} \leq M\left\{\sum_{s=1}^{N-1}\left\|A^{-1 / 2} f_{s}\right\|_{H} \tau+\left\|A^{-1 / 2} \psi\right\|_{H}+\|\varphi\|_{H}\right\}, \\
k=0,2, \ldots, N, \\
\left\|u_{1}\right\|_{H} \leq M\left[\sum_{s=1}^{N-1}\left\|A^{-1 / 2} f_{s}\right\|_{H} \tau+\|\varphi\|_{H}\right. \\
\left.+\left\|\left(I+i \tau A^{1 / 2}\right) A^{-1 / 2} \psi\right\|_{H}\right],
\end{gathered}
$$

$$
\begin{gathered}
\left\|A^{1 / 2} u_{k}\right\|_{H} \leq M\left\{\sum_{s=1}^{N-1}\left\|f_{s}\right\|_{H} \tau+\|\psi\|_{H}+\left\|A^{1 / 2} \varphi\right\|_{H}\right\} \\
k=0,2, \ldots, N \\
\left\|A^{1 / 2} u_{1}\right\|_{H} \\
\leq M\left[\sum_{s=1}^{N-1}\left\|f_{s}\right\|_{H} \tau+\left\|A^{1 / 2} \varphi\right\|_{H}+\left\|\left(I+i \tau A^{1 / 2}\right) \psi\right\|_{H}\right]
\end{gathered}
$$$$
\left\|A u_{k}\right\|_{H}
$$$$
\leq M\left\{\sum_{s=2}^{N-1}\left\|f_{s}-f_{s-1}\right\|_{H}+\left\|f_{1}\right\|_{H}\right.
$$$$
\left.+\left\|A^{1 / 2} \psi\right\|_{H}+\|A \varphi\|_{H}\right\}, \quad k=0,2, \ldots, N,
$$

$$
\begin{aligned}
\left\|A u_{1}\right\|_{H} \leq M\left[\sum_{s=2}^{N-1}\left\|f_{s}-f_{s-1}\right\|_{H}+\left\|f_{1}\right\|_{H}+\|A \varphi\|_{H}\right. \\
\left.+\left\|\left(I+i \tau A^{1 / 2}\right) A^{1 / 2} \psi\right\|_{H}\right]
\end{aligned}
$$

hold, where $M$ does not depend on $\tau, \varphi, \psi$, or $f_{s}, 1 \leq s \leq N-1$.

In [74], two types of second order of accuracy difference schemes

$$
\begin{gathered}
\tau^{-2}\left(u_{k+1}-2 u_{k}+u_{k-1}\right)+A u_{k}+\frac{\tau^{2}}{4} A^{2} u_{k+1}=f_{k}, \\
f_{k}=f\left(t_{k}\right), \\
t_{k}=k \tau, \quad 1 \leq k \leq N-1, \\
\left(I+\frac{\tau^{2} A}{2}\right) \tau^{-1}\left(u_{1}-u_{0}\right)-\frac{\tau}{2}\left(f_{0}-A u_{0}\right) \\
=\sum_{k=1}^{n} \beta_{k}\left\{\tau^{-1}\left(u_{\left[\lambda_{k} / \tau\right]}-u_{\left[\lambda_{k} / \tau\right]-1}\right)+\left(\frac{\tau}{2}+\left(\lambda_{k}-\left[\frac{\lambda_{k}}{\tau}\right] \tau\right)\right)\right. \\
\left.\cdot\left(f_{\left[\lambda_{k} / \tau\right]}-A u_{\left[\lambda_{k} / \tau\right]}\right)\right\}+\psi, \\
u_{0}=\sum_{m=1}^{n} \alpha_{m}\left\{u_{\left[\lambda_{m} / \tau\right]}+\tau^{-1}\left(u_{\left[\lambda_{m} / \tau\right]}-u_{\left[\lambda_{m} / \tau\right]-1}\right)\right. \\
\left.\cdot\left(\lambda_{m}-\left[\frac{\lambda_{m}}{\tau}\right] \tau\right)\right\}+\varphi, \\
f_{0}=f(0),
\end{gathered}
$$




$$
\begin{gathered}
\tau^{-2}\left(u_{k+1}-2 u_{k}+u_{k-1}\right)+\frac{1}{2} A u_{k} \\
+\frac{1}{4} A\left(u_{k+1}+u_{k-1}\right)=f_{k}, \\
f_{k}=f\left(t_{k}\right), \\
t_{k}=k \tau, \quad 1 \leq k \leq N-1, \\
\left(I+\frac{\tau^{2} A}{4}\right)\left[\left(I+\frac{\tau^{2} A}{4}\right) \tau^{-1}\left(u_{1}-u_{0}\right)-\frac{\tau}{2}\left(f_{0}-A u_{0}\right)\right] \\
=\sum_{k=1}^{n} \beta_{k}\left\{\tau^{-1}\left(u_{\left[\lambda_{k} / \tau\right]}-u_{\left[\lambda_{k} / \tau\right]-1}\right)\right. \\
+\left(\frac{\tau}{2}+\left(\lambda_{k}-\left[\frac{\lambda_{k}}{\tau}\right] \tau\right)\right) \\
\left.\cdot\left(f_{\left[\lambda_{k} / \tau\right]}-A u_{\left[\lambda_{k} / \tau\right]}\right)\right\}+\psi, \\
u_{0}=\sum_{m=1}^{n} \alpha_{m}\left\{u_{\left[\lambda_{m} / \tau\right]}+\tau^{-1}\left(u_{\left[\lambda_{m} / \tau\right]}-u_{\left[\lambda_{m} / \tau\right]-1}\right)\right. \\
\left.\cdot\left(\lambda_{m}-\left[\frac{\lambda_{m}}{\tau}\right] \tau\right)\right\}+\varphi, \\
f_{0}=f(0)
\end{gathered}
$$

for approximately solving nonlocal boundary value problem (207) were constructed.

Theorem 97 (see [74]). Suppose that $\varphi \in D(A), \psi \in D\left(A^{1 / 2}\right)$, and the assumption

$$
\begin{aligned}
& \sum_{k=1}^{n}\left|\alpha_{k}\right|\left(1+\left|\lambda_{k}-\left[\frac{\lambda_{k}}{\tau}\right] \tau\right|\right) \\
& +\sum_{k=1}^{n}\left|\beta_{k}\right|\left(1+\left|\lambda_{k}-\left[\frac{\lambda_{k}}{\tau}\right] \tau\right|\right) \\
& +\sum_{k=1}^{n}\left|\alpha_{k}\right| \sum_{k=1}^{n}\left|\beta_{k}\right|\left(1+\left|\lambda_{k}-\left[\frac{\lambda_{k}}{\tau}\right] \tau\right|\right) \\
& +\sum_{k=1}^{n}\left|\alpha_{k}\right|\left|\lambda_{k}-\left[\frac{\lambda_{k}}{\tau}\right] \tau\right| \sum_{k=1}^{n}\left|\beta_{k}\right|\left|\lambda_{k}-\left[\frac{\lambda_{k}}{\tau}\right] \tau\right|<1
\end{aligned}
$$

is satisfied. Then, for the solution of difference scheme (215), the stability inequalities

$$
\begin{aligned}
& \max _{0 \leq k \leq N}\left\|u_{k}\right\|_{H} \\
& \quad \leq M\left\{\sum_{k=0}^{N-1}\left\|A^{-1 / 2} f_{k}\right\|_{H} \tau+\left\|A^{-1 / 2} \psi\right\|_{H}+\|\varphi\|_{H}\right\}, \\
& \max _{0 \leq k \leq N}\left\|A^{1 / 2} u_{k}\right\|_{H} \\
& \quad \leq M\left\{\sum_{k=0}^{N-1}\left\|f_{k}\right\|_{H} \tau+\left\|A^{1 / 2} \varphi\right\|_{H}+\|\psi\|_{H}\right\},
\end{aligned}
$$

$$
\begin{aligned}
\max _{1 \leq k \leq N-1}\left\|\tau^{-2}\left(u_{k+1}-2 u_{k}+u_{k-1}\right)\right\|_{H} \\
\quad+\max _{0 \leq k \leq N-1}\left\|A u_{k}+\frac{\tau^{2} A^{2}}{4} u_{k+1}\right\|_{H} \\
\leq M\left\{\sum_{k=1}^{N-1}\left\|f_{k}-f_{k-1}\right\|_{H}+\left\|f_{0}\right\|_{H}+\left\|A^{1 / 2} \psi\right\|_{H}+\|A \varphi\|_{H}\right\}
\end{aligned}
$$

hold, where $M$ does not depend on $\tau, \varphi$, $\psi$, or $f_{k}, 0 \leq k \leq N-1$.

Theorem 98 (see [74]). Suppose that $\varphi \in D(A), \psi \in D\left(A^{1 / 2}\right)$, and assumption (217) holds. Then, for the solution of difference scheme (216), the stability inequalities

$$
\begin{aligned}
& \max _{0 \leq k \leq N}\left\|u_{k}\right\|_{H} \leq M\left\{\sum_{k=0}^{N-1}\left\|A^{-1 / 2} f_{k}\right\|_{H} \tau+\left\|A^{-1 / 2} \psi\right\|_{H}+\|\varphi\|_{H}\right\}, \\
& \max _{0 \leq k \leq N}\left\|A^{1 / 2} u_{k}\right\|_{H} \\
& \leq M\left\{\sum_{k=0}^{N-1}\left\|f_{k}\right\|_{H} \tau+\left\|A^{1 / 2} \varphi\right\|_{H}+\|\psi\|_{H}\right\}, \\
& \max _{1 \leq k \leq N-1}\left\|\tau^{-2}\left(u_{k+1}-2 u_{k}+u_{k-1}\right)\right\|_{H} \\
&+\max _{1 \leq k \leq N-1}\left\|\frac{1}{2} A u_{k}+\frac{1}{4} A\left(u_{k+1}+u_{k-1}\right)\right\|_{H} \\
& \leq M\left\{\sum_{k=1}^{N-1}\left\|f_{k}-f_{k-1}\right\|_{H}+\left\|f_{0}\right\|_{H}\right. \\
&\left.+\left\|A^{1 / 2} \psi\right\|_{H}+\|A \varphi\|_{H}\right\}
\end{aligned}
$$

hold, where $M$ does not depend on $\tau, \varphi, \psi$, or $f_{k}, 0 \leq k \leq N-1$.

In practice, difference schemes of the first and second order of accuracy difference schemes for the approximate solution of problems (210) and (211) were presented in [72, 74]. The stability estimates for the solution of these problems were established. Numerical experiments provide convincing support for the theoretical statements.

In [76], the third order of accuracy difference scheme

$$
\begin{gathered}
\tau^{-2}\left(u_{k+1}-2 u_{k}+u_{k-1}\right)+\frac{2}{3} A u_{k} \\
+\frac{1}{6} A\left(u_{k+1}+u_{k-1}\right)+\frac{1}{12} \tau^{2} A^{2} u_{k+1}=f_{k}, \\
f_{k}=\frac{2}{3} f\left(t_{k}\right)+\frac{1}{6}\left(f\left(t_{k+1}\right)+f\left(t_{k-1}\right)\right) \\
-\frac{1}{12} \tau^{2}\left(-A f\left(t_{k+1}\right)+f^{\prime \prime}\left(t_{k+1}\right)\right), \\
t_{k}=k \tau, \quad 1 \leq k \leq N-1, N \tau=1,
\end{gathered}
$$




$$
\begin{aligned}
& \left(I-i \tau A^{2}\right) u_{0} \\
& =\sum_{k=1}^{n} \alpha_{k}\left\{u_{\left[\lambda_{k} / \tau\right]}+\tau^{-1}\left(u_{\left[\lambda_{k} / \tau\right]}-u_{\left[\lambda_{k} / \tau\right]-1}\right)\right. \\
& \cdot\left(\lambda_{k}-\left[\frac{\lambda_{k}}{\tau}\right] \tau\right) \\
& +\frac{3}{2}\left(f_{\left[\lambda_{k} / \tau\right]}-A u_{\left[\lambda_{k} / \tau\right]}\right)\left(\lambda_{k}-\left[\frac{\lambda_{k}}{\tau}\right] \tau\right)^{2} \\
& +\frac{7}{6}\left(f_{\left[\lambda_{k} / \tau\right]}^{\prime}-\tau^{-1} A\left(u_{\left[\lambda_{k} / \tau\right]}-u_{\left[\lambda_{k} / \tau\right]-1}\right)\right) \\
& \left.\cdot\left(\lambda_{k}-\left[\frac{\lambda_{k}}{\tau}\right] \tau\right)^{3}\right\}+\varphi \\
& \left(I+i \tau A^{2}\right) \tau^{-1}\left(u_{1}-u_{0}\right) \\
& =\sum_{k=1}^{n} \beta_{k}\left\{\tau^{-1}\left(u_{\left[\lambda_{k} / \tau\right]}-u_{\left[\lambda_{k} / \tau\right]-1}\right)\right. \\
& +\left(f_{\left[\lambda_{k} / \tau\right]}-A u_{[\lambda k / \tau]}\right)\left(\lambda_{k}-\left[\frac{\lambda_{k}}{\tau}\right] \tau\right) \\
& +\frac{1}{2 !}\left(f_{\left[\lambda_{k} / \tau\right]}^{\prime}-\tau^{-1} A\left(u_{\left[\lambda_{k} / \tau\right]}-u_{\left[\lambda_{k} / \tau\right]-1}\right)\right) \\
& \cdot\left(\lambda_{k}-\left[\frac{\lambda_{k}}{\tau}\right] \tau\right)^{2} \\
& +\frac{1}{3 !}\left(f_{\left[\lambda_{k} / \tau\right]}^{\prime \prime}-A f_{\left[\lambda_{k} / \tau\right]}+A^{2} u_{\left[\lambda_{k} / \tau\right]}\right) \\
& \left.\cdot\left(\lambda_{k}-\left[\frac{\lambda_{k}}{\tau}\right] \tau\right)^{3}\right\}
\end{aligned}
$$$$
+\psi+f_{1,1} \tau,
$$

$$
f_{1,1}=\frac{1}{2} f(0)+f^{\prime}(0) \frac{\tau}{6}
$$

and the fourth order of accuracy difference scheme

$$
\begin{gathered}
\tau^{-2}\left(u_{k+1}-2 u_{k}+u_{k-1}\right)+\frac{5}{6} A u_{k} \\
+\frac{1}{12} A\left(u_{k+1}+u_{k-1}\right)-\frac{\tau^{2}}{72} A^{2} u_{k} \\
+\frac{\tau^{2}}{144} A^{2}\left(u_{k+1}+u_{k-1}\right) \\
=\frac{5}{6} f\left(t_{k}\right)+\frac{1}{12}\left(f\left(t_{k+1}\right)+f\left(t_{k-1}\right)\right) \\
+\frac{\tau^{2}}{72}\left(-A f\left(t_{k}\right)+f^{\prime \prime}\left(t_{k}\right)\right) \\
-\frac{1}{144} \tau^{2}\left[-A\left(f\left(t_{k+1}\right)+f\left(t_{k-1}\right)\right)\right. \\
\left.\quad+f^{\prime \prime}\left(t_{k+1}\right)+f^{\prime \prime}\left(t_{k-1}\right)\right], \\
t_{k}=k \tau, \quad 1 \leq k \leq N-1,
\end{gathered}
$$

$$
\begin{aligned}
u_{0}=\left(I-\frac{i \tau A^{1 / 2}}{2}+\right. & \left.\frac{\tau^{2} A^{3}}{12}\right)^{-1} \\
\times \sum_{k=1}^{n} \alpha_{k}\{( & \left.\left(\frac{\lambda_{k}}{\tau}-\left[\frac{\lambda_{k}}{\tau}\right]\right)-\frac{7 A \tau^{2}}{6}\left(\frac{\lambda_{k}}{\tau}-\left[\frac{\lambda_{k}}{\tau}\right]\right)^{3}\right) \\
& \times\left(u_{\left[\lambda_{k} / \tau\right]}-u_{\left[\lambda_{k} / \tau\right]-1}\right) \\
& +\left(I-\frac{3 \tau^{2} A}{2}\left(\frac{\lambda_{k}}{\tau}-\left[\frac{\lambda_{k}}{\tau}\right]\right)^{2}\right. \\
& \left.+\frac{\tau^{4} A^{2}}{24}\left(\frac{\lambda_{k}}{\tau}-\left[\frac{\lambda_{k}}{\tau}\right]\right)^{4}\right) u_{\left[\lambda_{k} / \tau\right]} \\
& +\frac{3}{2} \tau^{2}\left(\frac{\lambda_{k}}{\tau}-\left[\frac{\lambda_{k}}{\tau}\right]\right)^{2} f_{\left[\lambda_{k} / \tau\right]} \\
& +\frac{7}{6} \tau^{3}\left(\frac{\lambda_{k}}{\tau}-\left[\frac{\lambda_{k}}{\tau}\right]\right)^{3} f_{\left[\lambda_{k} / \tau\right]}^{\prime} \\
& +\frac{1}{24} \tau^{4}\left(\frac{\lambda_{k}}{\tau}-\left[\frac{\lambda_{k}}{\tau}\right]\right)^{4} f_{\left[\lambda_{k} / \tau\right]}^{\prime \prime} \\
& \left.-\frac{1}{24} A \tau^{4}\left(\frac{\lambda_{k}}{\tau}-\left[\frac{\lambda_{k}}{\tau}\right]\right)^{4} f_{\left[\lambda_{k} / \tau\right]}\right\}+\varphi,
\end{aligned}
$$$$
\tau^{-1}\left(u_{1}-u_{0}\right)
$$$$
=\left(I-\frac{\tau^{2} A}{12}\right)\left(I+\frac{i \tau A^{1 / 2}}{2}+\frac{\tau^{2} A^{3}}{12}\right)^{-1}
$$$$
\text { . } \sum_{k=1}^{n} \beta_{k}\left\{\left[\frac{1}{\tau}-\frac{\tau A}{2}\left(\frac{\lambda_{k}}{\tau}-\left[\frac{\lambda_{k}}{\tau}\right]\right)^{2}\right.\right.
$$$$
\left.+\frac{\tau^{3} A^{2}}{24}\left(\frac{\lambda_{k}}{\tau}-\left[\frac{\lambda_{k}}{\tau}\right]\right)^{4}\right]
$$$$
\cdot\left(u_{\left[\lambda_{k} / \tau\right]}-u_{\left[\lambda_{k} / \tau\right]-1}\right)
$$$$
+\left(-A \tau\left(\frac{\lambda_{k}}{\tau}-\left[\frac{\lambda_{k}}{\tau}\right]\right)\right.
$$$$
\left.+\frac{A^{2} \tau^{3}}{6}\left(\frac{\lambda_{k}}{\tau}-\left[\frac{\lambda_{k}}{\tau}\right]\right)^{3}\right) u_{\left[\lambda_{k} / \tau\right]}
$$$$
+\tau f_{\left[\lambda_{k} / \tau\right]}\left(\frac{\lambda_{k}}{\tau}-\left[\frac{\lambda_{k}}{\tau}\right]\right)
$$$$
+\frac{\tau^{2}}{2} f_{\left[\lambda_{k} / \tau\right]}^{\prime}\left(\frac{\lambda_{k}}{\tau}-\left[\frac{\lambda_{k}}{\tau}\right]\right)^{2}
$$$$
+\frac{\tau^{3}}{6} f_{\left[\lambda_{k} / \tau\right]}^{\prime \prime}\left(\frac{\lambda_{k}}{\tau}-\left[\frac{\lambda_{k}}{\tau}\right]\right)^{3}
$$$$
+\frac{\tau^{4}}{24} f_{\left[\lambda_{k} / \tau\right]}^{\prime \prime \prime}\left(\frac{\lambda_{k}}{\tau}-\left[\frac{\lambda_{k}}{\tau}\right]\right)^{4}
$$$$
-\frac{A \tau^{3}}{6} f_{\left[\lambda_{k} / \tau\right]}\left(\frac{\lambda_{k}}{\tau}-\left[\frac{\lambda_{k}}{\tau}\right]\right)^{3}
$$$$
\left.-\frac{A \tau^{4}}{24} f_{\left[\lambda_{k} / \tau\right]}^{\prime}\left(\frac{\lambda_{k}}{\tau}-\left[\frac{\lambda_{k}}{\tau}\right]\right)^{4}\right\}
$$$$
+\psi+f_{2,2} \tau,
$$ 


$$
\begin{aligned}
f_{2,2}=\{( & \left.I-\frac{\tau^{2} A}{12}\right) f(0) \\
& +\left(-\left(I-\frac{5 \tau^{2} A}{12}\right) f(0)+\tau f^{\prime}(0)\right) \frac{1}{2} \\
& +\left(-A \tau f(0)-2 f^{\prime}(0)+\tau f^{\prime \prime}(0)\right) \frac{\tau}{6} \\
& \left.+\left(A f(0)-3 f^{\prime \prime}(0)\right) \frac{\tau^{2}}{24}\right\}
\end{aligned}
$$

for approximately solving multipoint nonlocal BVP (207) were constructed. The stability estimates for the solution of difference scheme (220) were obtained under the assumption

$$
\begin{gathered}
\sum_{k=1}^{n}\left|\alpha_{k}\right|\left\{1+\left|\frac{\lambda_{k}}{\tau}-\left[\frac{\lambda_{k}}{\tau}\right]\right|+\frac{3}{2}\left|\frac{\lambda_{k}}{\tau}-\left[\frac{\lambda_{k}}{\tau}\right]\right|^{2}\right. \\
\left.+\frac{7}{6}\left|\frac{\lambda_{k}}{\tau}-\left[\frac{\lambda_{k}}{\tau}\right]\right|^{3}+\frac{1}{24}\left|\frac{\lambda_{k}}{\tau}-\left[\frac{\lambda_{k}}{\tau}\right]\right|^{4}\right\} \\
+\sum_{k=1}^{n}\left|\beta_{k}\right|\left\{1+\left|\frac{\lambda_{k}}{\tau}-\left[\frac{\lambda_{k}}{\tau}\right]\right|+\frac{1}{2}\left|\frac{\lambda_{k}}{\tau}-\left[\frac{\lambda_{k}}{\tau}\right]\right|^{2}\right. \\
\left.+\frac{1}{6}\left|\frac{\lambda_{k}}{\tau}-\left[\frac{\lambda_{k}}{\tau}\right]\right|^{3}+\frac{1}{24}\left|\frac{\lambda_{k}}{\tau}-\left[\frac{\lambda_{k}}{\tau}\right]\right|^{4}\right\} \\
+\sum_{k=1}^{n}\left|\alpha_{k}\right| \sum_{k=1}^{n}\left|\beta_{k}\right| \\
\cdot\left\{1+\left|\frac{\lambda_{k}}{\tau}-\left[\frac{\lambda_{k}}{\tau}\right]\right|^{2}\right. \\
\left.+\frac{1}{576}\left|\frac{\lambda_{k}}{\tau}-\left[\frac{\lambda_{k}}{\tau}\right]\right|^{4}+\frac{1}{9}\left|\frac{\lambda_{k}}{\tau}-\left[\frac{\lambda_{k}}{\tau}\right]\right|^{6}\right\}<1
\end{gathered}
$$

Theorem 99 (see [76]). Suppose that assumption (222) holds and $\varphi \in D\left(A^{1 / 2}\right), \psi \in D\left(A^{1 / 2}\right)$. Then, for the solution of difference scheme (220), the following stability estimates hold:

$$
\begin{aligned}
\max _{0 \leq k \leq N}\left\|u_{k}\right\|_{H} & \\
\leq M & \left\{\sum_{s=1}^{N-1}\left\|A^{-1 / 2} f_{s}\right\|_{H} \tau+\|\varphi\|_{H}\right. \\
& \left.+\left\|A^{-1 / 2} \psi\right\|_{H}+\tau\left\|A^{-1 / 2} f_{1,1}\right\|_{H}\right\},
\end{aligned}
$$

$$
\begin{gathered}
\max _{0 \leq k \leq N}\left\|A^{1 / 2} u_{k}\right\|_{H} \\
\leq M\left\{\sum_{s=1}^{N-1}\left\|f_{s}\right\|_{H} \tau+\left\|A^{1 / 2} \varphi\right\|_{H}+\|\psi\|_{H}+\tau\left\|f_{1,1}\right\|_{H}\right\}, \\
\max _{0 \leq k \leq N}\left\|A u_{k}\right\|_{H} \\
\leq M\left\{\sum_{s=2}^{N-1}\left\|f_{s}-f_{s-1}\right\|_{H}+\left\|f_{1}\right\|_{H}+\|A \varphi\|_{H}\right. \\
\left.+\left\|A^{1 / 2} \psi\right\|_{H}+\tau\left\|A^{1 / 2} f_{1,1}\right\|_{H}\right\},
\end{gathered}
$$

where $M$ does not depend on $\tau, \varphi, \psi, f_{1,1}$, or $f_{s}, 1 \leq s \leq N-1$.

The stability estimates for the solution of difference scheme (221) were obtained under the assumption

$$
\begin{gathered}
\sum_{k=1}^{n}\left|\alpha_{k}\right|\left\{1+\left|\frac{\lambda_{k}}{\tau}-\left[\frac{\lambda_{k}}{\tau}\right]\right|+\frac{3}{2}\left|\frac{\lambda_{k}}{\tau}-\left[\frac{\lambda_{k}}{\tau}\right]\right|^{2}\right. \\
\left.+\frac{7}{6}\left|\frac{\lambda_{k}}{\tau}-\left[\frac{\lambda_{k}}{\tau}\right]\right|^{3}+\left.\frac{1}{24}\left|\frac{\lambda_{k}}{\tau}-\left[\frac{\lambda_{k}}{\tau}\right]\right|\right|^{4}\right\} \\
+\sum_{k=1}^{n}\left|\beta_{k}\right|\left\{1+\left|\frac{\lambda_{k}}{\tau}-\left[\frac{\lambda_{k}}{\tau}\right]\right|+\frac{1}{2}\left|\frac{\lambda_{k}}{\tau}-\left[\frac{\lambda_{k}}{\tau}\right]\right|^{2}\right. \\
\left.+\frac{1}{6}\left|\frac{\lambda_{k}}{\tau}-\left[\frac{\lambda_{k}}{\tau}\right]\right|^{3}+\frac{1}{24}\left|\frac{\lambda_{k}}{\tau}-\left[\frac{\lambda_{k}}{\tau}\right]\right|^{4}\right\} \\
+\sum_{k=1}^{n}\left|\alpha_{k}\right| \sum_{k=1}^{n}\left|\beta_{k}\right| \\
+\left\{1+\left|\frac{\lambda_{k}}{\tau}-\left[\frac{\lambda_{k}}{\tau}\right]\right|^{2}+\frac{1}{2}\left|\frac{\lambda_{k}}{\tau}-\left[\frac{\lambda_{k}}{\tau}\right]\right|^{4}\right. \\
+\frac{1}{9}\left|\frac{\lambda_{k}}{\tau}-\left[\frac{\lambda_{k}}{\tau}\right]\right|^{6} \\
\left.+\frac{\lambda_{k}}{\tau}-\left.\left[\frac{\lambda_{k}}{\tau}\right]\right|^{8}\right\}<1
\end{gathered}
$$

Theorem 100 (see [76]). Suppose that assumption (224) holds and $\varphi \in D\left(A^{1 / 2}\right), \psi \in D\left(A^{1 / 2}\right)$. Then, for the solution of difference scheme (221), the following stability estimates hold:

$$
\begin{aligned}
\max _{2 \leq k \leq N} \| & \frac{u_{k}+u_{k-1}}{2} \|_{H} \\
\leq M & \left\{\sum_{s=1}^{k-1}\left\|A^{-1 / 2} f_{s}\right\|_{H} \tau+\|\varphi\|_{H}\right. \\
& \left.+\left\|A^{-1 / 2} \psi\right\|_{H}+\tau\left\|A^{-1 / 2} f_{2,2}\right\|_{H}\right\},
\end{aligned}
$$




$$
\begin{aligned}
& \left\|u_{1}\right\|_{H} \leq M\left\{\sum_{s=1}^{k-1}\left\|A^{-1 / 2} f_{s}\right\|_{H} \tau+\|\varphi\|_{H}\right. \\
& \left.+\left\|A^{-1 / 2} \psi\right\|_{H}+\tau\left\|A^{-1 / 2} f_{2,2}\right\|_{H}\right\}, \\
& \max _{2 \leq k \leq N-1}\left\|\frac{u_{k+1}-u_{k-1}}{2 \tau}\right\|_{H}+\max _{2 \leq k \leq N}\left\|A^{1 / 2} \frac{u_{k}+u_{k-1}}{2}\right\|_{H} \\
& \leq M\left\{\sum_{s=1}^{k-1}\left\|f_{s}\right\|_{H} \tau+\left\|A^{1 / 2} \varphi\right\|_{H}+\|\psi\|_{H}+\tau\left\|f_{2,2}\right\|_{H}\right\}, \\
& \left\|A^{1 / 2} u_{1}\right\|_{H} \\
& \leq M\left\{\sum_{s=1}^{k-1}\left\|f_{s}\right\|_{H} \tau+\left\|A^{1 / 2} \varphi\right\|_{H}+\|\psi\|_{H}+\tau\left\|f_{2,2}\right\|_{H}\right\}, \\
& \max _{2 \leq k \leq N-1}\left\|A^{1 / 2} \frac{u_{k+1}-u_{k-1}}{2 \tau}\right\|_{H}+\max _{2 \leq k \leq N}\left\|A \frac{u_{k}+u_{k-1}}{2}\right\|_{H} \\
& \leq M\left\{\sum_{s=2}^{N-1}\left\|f_{s}-f_{s-1}\right\|_{H}+\left\|f_{1}\right\|_{H}\right. \\
& \left.+\|A \varphi\|_{H}+\left\|A^{1 / 2} \psi\right\|_{H}+\tau\left\|A^{1 / 2} f_{2,2}\right\|_{H}\right\}, \\
& \left\|A u_{1}\right\|_{H} \leq M\left\{\sum_{s=2}^{N-1}\left\|f_{s}-f_{s-1}\right\|_{H}+\left\|f_{1}\right\|_{H}\right. \\
& \left.+\|A \varphi\|_{H}+\left\|A^{1 / 2} \psi\right\|_{H}+\tau\left\|A^{1 / 2} f_{2,2}\right\|_{H}\right\},
\end{aligned}
$$

where $M$ does not depend on $\tau, \varphi, \psi, f_{2,2}$, or $f_{s}, 1 \leq s \leq N-1$.

In $[70,71]$, the nonlocal boundary value problem for the multidimensional hyperbolic equation

$$
\begin{array}{r}
\frac{\partial^{2} u(t, x)}{\partial t^{2}}-\sum_{r=1}^{m}\left(a_{r}(x) u_{x_{r}}\right)_{x_{r}}+\sigma u(t, x)=f(t, x), \\
x=\left(x_{1}, \ldots, x_{m}\right) \in \Omega, \quad 0<t<T,
\end{array}
$$

with integral conditions

$$
\begin{aligned}
u(0, x)= & \int_{0}^{T} \alpha(\rho) u(\rho, x) d \rho \\
& +\sum_{i=1}^{n} a\left(\lambda_{i}\right) u\left(\lambda_{i}, x\right)+\varphi(x), \quad x \in \bar{\Omega}, \\
u_{t}(0, x)= & \int_{0}^{T} \beta(\rho) u_{t}(\rho, x) d \rho \\
& +\sum_{i=1}^{n} b\left(\lambda_{i}\right) u_{t}\left(\lambda_{i}, x\right)+\psi(x), \quad x \in \bar{\Omega},
\end{aligned}
$$

and nonclassical conditions

$$
\begin{gathered}
\left.u(t, x)\right|_{S_{1}}=0, \\
\left.\frac{\partial u(t, x)}{\partial \vec{n}}\right|_{S_{2}}=0, \quad 0 \leq t \leq T,
\end{gathered}
$$

or classical Dirichlet condition

$$
\left.u\right|_{S}=0
$$

or classical Neumann condition

$$
\left.\frac{\partial u(t, x)}{\partial \vec{n}}\right|_{S}=0
$$

was investigated under the assumption

$$
\begin{aligned}
\mid 1+ & \int_{0}^{T} \alpha(s) \beta(s) d s+\sum_{k=1}^{n} a\left(\lambda_{k}\right) \sum_{k=1}^{n} b\left(\lambda_{k}\right) \\
& +\sum_{k=1}^{n} a\left(\lambda_{k}\right) \int_{0}^{T} \beta(s) d s+\sum_{k=1}^{n} b\left(\lambda_{k}\right) \int_{0}^{T} \alpha(s) d s \mid \\
> & \int_{0}^{T}(|\alpha(s)|+|\beta(s)|) d s \\
& +\sum_{k=1}^{n}\left|a\left(\lambda_{k}\right)+b\left(\lambda_{k}\right)\right| .
\end{aligned}
$$

Here, $\Omega$ is the unit open cube in the $m$-dimensional Euclidean space $\mathbb{R}^{m}\left(x=\left(x_{1}, \ldots, x_{m}\right): 0<x_{j}<1,1 \leq j \leq\right.$ $m)$ with boundary $S=S_{1} \cup S_{2}, \bar{\Omega}=\Omega \cup S, a_{r}(x)(x \in$ $\Omega), \varphi(x), \psi(x)(x \in \bar{\Omega})$, and $f(t, x)(t \in(0, T), x \in \Omega)$ which are given smooth functions, and $a_{r}(x) \geq a>0$. $\vec{n}$ is the normal vector to $\Omega, \sigma \geq 0$, and $\alpha(s), \beta(s), a(s)$, and $b(s)$ are scalar real-valued continuous functions. Theorems on stability of solutions of these problems were established. The first and second order of accuracy in $t$ stable difference schemes for the approximate solution of these problems were presented. Stability of these difference schemes was obtained under the assumption

$$
1>\sum_{j=1}^{N}\left|\alpha\left(t_{j}\right)\right| \tau \sum_{j=1}^{N}\left|\beta\left(t_{j}\right)\right| \tau+\sum_{j=1}^{N}\left|\alpha\left(t_{j}\right)\right| \tau+\sum_{j=1}^{N}\left|\beta\left(t_{j}\right)\right| \tau
$$

for the first order difference scheme and under the assumption

$$
\begin{array}{r}
1>\sum_{j=1}^{N}\left|\alpha\left(t_{j}-\frac{\tau}{2}\right)\right| \tau+\sum_{j=1}^{N}\left|\beta\left(t_{j}-\frac{\tau}{2}\right)\right| \tau \\
+\sum_{j=1}^{N}\left|\alpha\left(t_{j}-\frac{\tau}{2}\right)\right| \tau \sum_{j=1}^{N}\left|\beta\left(t_{j}-\frac{\tau}{2}\right)\right| \tau
\end{array}
$$

for the second order difference scheme.

The theoretical statements for the solution of these difference schemes for one-dimensional hyperbolic equations are supported by two numerical examples in computer. We show that the second order of accuracy difference scheme is more accurate compared with the first order of accuracy difference scheme. 
7.4. Hyperbolic and Mixed Type PDEs. The solutions of large-scale scientific-technological problems in the field of construction of the base of project and system of rational elaboration, improvement of elaboration and exploitation of technology, modern methods of construction of deep well-holes in complicated conditions, hydrodynamics of low permeable spaces, and protection of environment become possible only owing to the application of mathematical models and new numerical methods implemented on computers. Mathematical models of many problems of such type are reduced to nonclassical or classical problems of mixed type PDEs (see, e.g., [121-125]). Ashyralyev jointly with his group of scientists investigated the modeling processes of exploitation of gas places. The investigation of the underground natural gas beneath the earth $6 \mathrm{~km}$ from the underlying is based on mathematical models. In this section we give results of his group on the stability of nonlocal problems for partial differential equations of mixed hyperbolic-parabolic and elliptic-hyperbolic types. First, we consider the nonlocal boundary value problem

$$
\begin{gathered}
\frac{d^{2} u(t)}{d t^{2}}+A u(t)=f(t) \quad(0 \leq t \leq T), \\
\frac{d u(t)}{d t}+A u(t)=g(t) \quad(-T \leq t \leq 0), \\
u(-T)=\alpha u(\mu)+\varphi, \quad 0 \leq \alpha \leq 1,0<\mu \leq T,
\end{gathered}
$$

for hyperbolic-parabolic differential equations of mixed type in Hilbert space $H$, where $A=A^{*} \geq \delta I(\delta>0)$ is a positive definite and self-adjoint operator with dense domain $D(A)$.

Function $u(t)$ is called a solution of problem (234) if the following conditions are satisfied:

(i) $u(t)$ is twice continuously differentiable on the interval $(0, T]$ and continuously differentiable on the segment $[-T, T]$.

(ii) The element $u(t)$ belongs to $D(A)$ for all $t \in[-T, T]$, and the function $A u(t)$ is continuous on the segment $[-T, T]$.

(iii) $u(t)$ satisfies the equations and nonlocal boundary condition (234).

Theorem 101. Suppose that $\varphi \in D(A)$ and $f(t)$ are continuously differentiable on $[0, T]$ and $g(t)$ is continuously differentiable on $[-T, 0]$ function. Then there is a unique solution of problem (234) and the stability inequalities

$$
\begin{aligned}
& \max _{-T \leq t \leq T}\|u(t)\|_{H} \\
& \quad \leq M\left[\|\varphi\|_{H}+\max _{-T \leq t \leq 0}\|g(t)\|_{H}+\max _{0 \leq t \leq T}\left\|A^{-1 / 2} f(t)\right\|_{H}\right], \\
& \max _{-T \leq t \leq T}\left\|A^{1 / 2} u(t)\right\|_{H} \\
& \quad \leq M\left[\left\|A^{1 / 2} \varphi\right\|_{H}+\int_{-T}^{0}\|g(t)\|_{H} d t+\max _{0 \leq t \leq T}\|f(t)\|_{H}\right],
\end{aligned}
$$

$$
\begin{aligned}
\max _{-T \leq t \leq 0}\left\|\frac{d u(t)}{d t}\right\|_{H}+\max _{0 \leq t \leq T}\left\|\frac{d^{2} u(t)}{d t^{2}}\right\|_{H}+\max _{-T \leq t \leq T}\|A u(t)\|_{H} \\
\leq M\left[\|A \varphi\|_{H}+\left\|A^{1 / 2} g(0)\right\|_{H}\right. \\
\left.+\|f(0)\|_{H}+\max _{-T \leq t \leq 0}\left\|g^{\prime}(t)\right\|_{H}+\int_{0}^{T}\left\|f^{\prime}(t)\right\|_{H} d t\right]
\end{aligned}
$$

hold, where $M$ does not depend on $f(t), t \in[0, T], g(t) \in$ $[-T, 0]$, or $\varphi$.

In [87], the difference analogues of these stability inequalities were presented for solutions of the first order of accuracy difference scheme

$$
\begin{gathered}
\tau^{-2}\left(u_{k+1}-2 u_{k}+u_{k-1}\right)+A u_{k+1}=f_{k}, \\
f_{k}=f\left(t_{k}\right), \\
t_{k}=k \tau, \quad 1 \leq k \leq N-1, \\
\tau^{-1}\left(u_{1}-u_{0}\right)+i A^{1 / 2} u_{1}=\left(-A+i A^{1 / 2}\right) u_{0}+g_{0}, \\
N \tau=T, \\
\tau^{-1}\left(u_{k}-u_{k-1}\right)+A u_{k}=g_{k}, \\
g_{k}=g\left(t_{k}\right), \\
t_{k}=k \tau, \quad-(N-1) \leq k \leq 0, \\
u_{-N}=\alpha u_{[\mu / \tau]}+\varphi
\end{gathered}
$$

and second order of accuracy of the two types of difference schemes

$$
\begin{gathered}
\tau^{-2}\left(u_{k+1}-2 u_{k}+u_{k-1}\right)+A u_{k}+\frac{\tau^{2}}{4} A^{2} u_{k+1}=f\left(t_{k}\right), \\
t_{k}=k \tau, \quad 1 \leq k \leq N-1, \\
\tau^{-1}\left(u_{1}-u_{0}\right)+i A^{1 / 2}\left(I+i \frac{\tau A^{1 / 2}}{2}\right) u_{1}=Z_{1}, \\
N \tau=T, \\
Z_{1}=\frac{\tau}{2}\left(f(0)-A u_{0}\right)+\left(i A^{1 / 2}-\tau A\right) u_{0} \\
+\left(I-i \tau A^{1 / 2}\right)\left(g(0)-A u_{0}\right), \\
\tau^{-1}\left(u_{k}-u_{k-1}\right)+A\left(I+\frac{\tau}{2} A\right) u_{k}=\left(I+\frac{\tau}{2} A\right) g\left(t_{k}-\frac{\tau}{2}\right),
\end{gathered}
$$




$$
\begin{aligned}
& t_{k}=k \tau, \quad-(N-1) \leq k \leq 0, \\
& u_{-N}=\alpha\left(u_{[\mu / \tau]}+\left(u_{[\mu / \tau]+1}-u_{[\mu / \tau]}\right)\left(\frac{\mu}{\tau}-\left[\frac{\mu}{\tau}\right]\right)\right)+\varphi \\
& \tau^{-2}\left(u_{k+1}-2 u_{k}+u_{k-1}\right)+\frac{1}{2} A u_{k} \\
& +\frac{1}{4} A\left(u_{k+1}+u_{k-1}\right)=f\left(t_{k}\right) \\
& t_{k}=k \tau, \quad 1 \leq k \leq N-1, \\
& \tau^{-1}\left(u_{1}-u_{0}\right)+i A^{1 / 2}\left(I+i \frac{\tau A^{1 / 2}}{2}\right) u_{1}=Z_{1} \\
& N \tau=1, \\
& Z_{1}=\frac{\tau}{2}\left(f(0)-A u_{0}\right)+\left(i A^{1 / 2}-\tau A\right) u_{0} \\
& +\left(I-i \tau A^{1 / 2}\right)\left(g(0)-A u_{0}\right), \\
& \tau^{-1}\left(u_{k}-u_{k-1}\right)+A\left(I+\frac{\tau}{2} A\right) u_{k}=\left(I+\frac{\tau}{2} A\right) g\left(t_{k}-\frac{\tau}{2}\right), \\
& t_{k}=k \tau, \quad-(N-1) \leq k \leq 0, \\
& u_{-N}=\alpha\left(u_{[\mu / \tau]}+\left(u_{[\mu / \tau]+1}-u_{[\mu / \tau]}\right)\left(\frac{\mu}{\tau}-\left[\frac{\mu}{\tau}\right]\right)\right)+\varphi
\end{aligned}
$$

for approximately solving boundary value problem (234).

However, for the practical realization of these difference schemes, it is necessary to first construct operator $A^{1 / 2}$. This action is very difficult for a computer. Therefore, in spite of the theoretical results, the role of their application to the numerical solution of the boundary value problem is not great.

Let us associate with boundary value problem (234) the corresponding first order of accuracy difference scheme

$$
\begin{gathered}
\tau^{-2}\left(u_{k+1}-2 u_{k}+u_{k-1}\right)+A u_{k+1}=f_{k}, \\
f_{k}=f\left(t_{k}\right), \\
t_{k}=k \tau, \quad 1 \leq k \leq N-1, \\
\tau^{-1}\left(u_{1}-u_{0}\right)=-A u_{0}+g_{0}, \\
N \tau=T \\
\tau^{-1}\left(u_{k}-u_{k-1}\right)+A u_{k}=g_{k}, \\
g_{k}=g\left(t_{k}\right) \\
-(N-1) \leq k \leq 0 \\
t_{k}=k \tau, \quad\left(N-\alpha u_{[\mu / \tau]}+\varphi .\right.
\end{gathered}
$$

Theorem 102. Let $\varphi \in D(A), g_{0} \in D\left(A^{1 / 2}\right)$. Then for the solution of difference scheme (238) the stability inequalities

$$
\begin{aligned}
& \max _{-N \leq k \leq N}\left\|u_{k}\right\|_{H} \\
& \leq M\left[\max _{1 \leq k \leq N-1}\left\|A^{-1 / 2} f_{k}\right\|_{H}+\max _{-(N-1) \leq k \leq 0}\left\|g_{k}\right\|_{H}+\|\varphi\|_{H}\right], \\
& \max _{-N \leq k \leq N}\left\|A^{1 / 2} u_{k}\right\|_{H} \\
& \leq M\left[\max _{1 \leq k \leq N-1}\left\|f_{k}\right\|_{H}+\left\|A^{1 / 2} \varphi\right\|_{H}+\left\|g_{0}\right\|_{H}\right. \\
& \left.+\sum_{k=-(N-1)}^{0}\left\|g_{k}-g_{k-1}\right\|_{H}\right] \\
& \max _{1 \leq k \leq N-1}\left\|\tau^{-2}\left(u_{k+1}-2 u_{k}+u_{k-1}\right)\right\|_{H} \\
& +\max _{-(N-1) \leq k \leq 0}\left\|\tau^{-1}\left(u_{k}-u_{k-1}\right)\right\|_{H}+\max _{-N \leq k \leq N}\left\|A u_{k}\right\|_{H} \\
& \leq M\left[\sum_{k=2}^{N-1}\left\|f_{k}-f_{k-1}\right\|_{H}+\left\|f_{1}\right\|_{H}\right. \\
& +\left\|A^{1 / 2} g_{0}\right\|_{H}+\|A \varphi\|_{H} \\
& \left.+\max _{-(N-1) \leq k \leq 0}\left\|\left(g_{k}-g_{k-1}\right) \tau^{-1}\right\|_{H}\right]
\end{aligned}
$$

hold, where $M$ does not depend on $f_{k}, g_{k}$, or $\varphi$.

Note that Theorem 102 permits us to obtain the convergence estimates for the solution of difference scheme (238).

In $[88,89]$, the same stability results for the solution of the following difference schemes of second order of accuracy

$$
\begin{gathered}
\tau^{-2}\left(u_{k+1}-2 u_{k}+u_{k-1}\right)+A u_{k}+\frac{\tau^{2}}{4} A^{2} u_{k+1}=f\left(t_{k}\right), \\
t_{k}=k \tau, \quad 1 \leq k \leq N-1, \\
\tau^{-1}\left(I+\tau^{2} A\right)\left(u_{1}-u_{0}\right)=Z_{1}, \\
N \tau=T, \\
Z_{1}=\frac{\tau}{2}\left(f(0)-A u_{0}\right)+\left(g(0)-A u_{0}\right),
\end{gathered}
$$




$$
\begin{gathered}
\tau^{-1}\left(u_{k}-u_{k-1}\right)+A\left(I+\frac{\tau}{2} A\right) u_{k}=\left(I+\frac{\tau}{2} A\right) g\left(t_{k}-\frac{\tau}{2}\right), \\
t_{k}=k \tau, \quad-(N-1) \leq k \leq 0, \\
u_{-N}=\alpha\left(u_{[\mu / \tau]}+\left(u_{[\mu / \tau]+1}-u_{[\mu / \tau]}\right)\left(\frac{\mu}{\tau}-\left[\frac{\mu}{\tau}\right]\right)\right)+\varphi ; \\
\tau^{-2}\left(u_{k+1}-2 u_{k}+u_{k-1}\right)+\frac{1}{2} A u_{k} \\
+\frac{1}{4} A\left(u_{k+1}+u_{k-1}\right)=f\left(t_{k}\right), \\
t_{k}=k \tau, \quad 1 \leq k \leq N-1, \\
\tau^{-1}\left(I+\tau^{2} A\right)\left(u_{1}-u_{0}\right)=Z_{1}, \\
N \tau=T, \\
Z_{1}=\frac{\tau}{2}\left(f(0)-A u_{0}\right)+\left(g(0)-A u_{0}\right), \\
\tau^{-1}\left(u_{k}-u_{k-1}\right)+A\left(I+\frac{\tau}{2} A\right) u_{k}=\left(I+\frac{\tau}{2} A\right) g\left(t_{k}-\frac{\tau}{2}\right), \\
t_{k}=k \tau, \quad-(N-1) \leq k \leq 0, \\
u_{-N}=\alpha\left(u_{[\mu / \tau]}+\left(u_{[\mu / \tau]+1}-u_{[\mu / \tau]}\right)\left(\frac{\mu}{\tau}-\left[\frac{\mu}{\tau}\right]\right)\right)+\varphi,
\end{gathered}
$$

for approximately solving problem (234), were obtained. Moreover, the nonlocal boundary value problem

$$
\begin{gathered}
\frac{d^{2} u(t)}{d t^{2}}+A u(t)=f(t, u(t)) \quad(0 \leq t \leq T), \\
\frac{d u(t)}{d t}+A u(t)=g(t, u(t)) \quad(-T \leq t \leq 0), \\
u(-T)=\alpha u(\mu)+\varphi, \quad 0 \leq \alpha \leq 1,0<\mu \leq T,
\end{gathered}
$$

for semilinear differential equations of mixed type in Hilbert space $H$ with an operator $A=A^{*} \geq \delta I$ was considered. The first order of accuracy

$$
\begin{gathered}
\tau^{-2}\left(u_{k+1}-2 u_{k}+u_{k-1}\right)+A u_{k+1}=f\left(t_{k}, u_{k}\right), \\
t_{k}=k \tau, \quad 1 \leq k \leq N-1, \\
\tau^{-1}\left(u_{1}-u_{0}\right)=-A u_{0}+g\left(0, u_{0}\right), \\
N \tau=T, \\
\tau^{-1}\left(u_{k}-u_{k-1}\right)+A u_{k}=g\left(t_{k}, u_{k}\right), \\
t_{k}=k \tau, \quad-(N-1) \leq k \leq 0, \\
u_{-N}=\alpha u_{[\mu / \tau]}+\varphi
\end{gathered}
$$

and second order of accuracy

$$
\begin{gathered}
\tau^{-2}\left(u_{k+1}-2 u_{k}+u_{k-1}\right)+A u_{k}+\frac{\tau^{2}}{4} A^{2} u_{k+1}=f\left(t_{k}, u_{k}\right), \\
t_{k}=k \tau, \quad 1 \leq k \leq N-1, \\
\tau^{-1}\left(I+\tau^{2} A\right)\left(u_{1}-u_{0}\right)=Z_{1}, \\
N \tau=T, \\
Z_{1}=\frac{\tau}{2}\left(f\left(0, u_{0}\right)-A u_{0}\right)+\left(g\left(0, u_{0}\right)-A u_{0}\right), \\
\tau^{-1}\left(u_{k}-u_{k-1}\right)+A\left(I+\frac{\tau}{2} A\right) u_{k} \\
=\left(I+\frac{\tau}{2} A\right) g\left(t_{k}-\frac{\tau}{2}, \frac{1}{2}\left(u_{k}+u_{k-1}\right)\right), \\
t_{k}=k \tau, \quad-(N-1) \leq k \leq 0,
\end{gathered}
$$$$
u_{-N}=\alpha\left(u_{[\mu / \tau]}+\left(u_{[\mu / \tau]+1}-u_{[\mu / \tau]}\right)\left(\frac{\mu}{\tau}-\left[\frac{\mu}{\tau}\right]\right)\right)+\varphi ;
$$$$
\tau^{-2}\left(u_{k+1}-2 u_{k}+u_{k-1}\right)+\frac{1}{2} A u_{k}
$$$$
+\frac{1}{4} A\left(u_{k+1}+u_{k-1}\right)=f\left(t_{k}, u_{k}\right),
$$$$
t_{k}=k \tau, \quad 1 \leq k \leq N-1,
$$$$
\tau^{-1}\left(I+\tau^{2} A\right)\left(u_{1}-u_{0}\right)=Z_{1} \text {, }
$$$$
N \tau=T \text {, }
$$$$
Z_{1}=Z_{1}=\frac{\tau}{2}\left(f\left(0, u_{0}\right)-A u_{0}\right)+\left(g\left(0, u_{0}\right)-A u_{0}\right),
$$$$
\tau^{-1}\left(u_{k}-u_{k-1}\right)+A\left(I+\frac{\tau}{2} A\right) u_{k}
$$$$
=\left(I+\frac{\tau}{2} A\right) g\left(t_{k}-\frac{\tau}{2}, \frac{1}{2}\left(u_{k}+u_{k-1}\right)\right),
$$$$
t_{k}=k \tau, \quad-(N-1) \leq k \leq 0,
$$$$
u_{-N}=\alpha\left(u_{[\mu / \tau]}+\left(u_{[\mu / \tau]+1}-u_{[\mu / \tau]}\right)\left(\frac{\mu}{\tau}-\left[\frac{\mu}{\tau}\right]\right)\right)+\varphi
$$

difference schemes approximately solving problem (241) were investigated. The convergence estimates for the solution of these difference schemes were obtained. Abstract theorems of [87-89] permit us to obtain the stability estimates for the solutions of these difference schemes. A finite difference method and some results of numerical experiments are presented in order to support theoretical statements. 
Such type stability results for special cases of hyperbolicparabolic equations were obtained before in [78-81].

In $[82,83]$, the nonlocal boundary value problem

$$
\begin{gathered}
\frac{d^{2} u(t)}{d t^{2}}+A u(t)=f(t) \quad(0 \leq t \leq T), \\
u(-T)=\alpha u(\mu)+\beta u^{\prime}(\lambda)+\varphi, \\
\frac{d u(t)}{d t}+A u(t)=g(t) \quad(-T \leq t \leq 0), \\
|\alpha| \leq 1, \quad 0<|\beta| \leq 1, \\
0<\mu, \quad \lambda \leq T,
\end{gathered}
$$

for hyperbolic-parabolic differential equations of mixed type in Hilbert space $H$, with a positive definite and self-adjoint operator $A=A^{*} \geq \delta I(\delta>0)$ with dense domain $D(A)$, was considered.

Function $u(t)$ is called a solution of problem (244) if the following conditions are satisfied:

(i) $u(t)$ is twice continuously differentiable on the interval $(0, T]$ and continuously differentiable on the segment $[-T, T]$.

(ii) The element $u(t)$ belongs to $D(A)$ for all $t \in[-T, T]$, and the function $A u(t)$ is continuous on the segment $[-T, T]$.

(iii) $u(t)$ satisfies the equations and nonlocal boundary condition (244).

Theorem 103 (see [83]). Suppose that $\varphi \in D(A), g(0) \in$ $D\left(A^{1 / 2}\right), g^{\prime}(0) \in H, f(0) \in D\left(A^{1 / 2}\right)$, and $f^{\prime}(0) \in H$. Let $f(t)$ be twice continuously differentiable on $[0, T]$ and let $g(t)$ be twice continuously differentiable on $[-T, 0]$ functions. Then there is a unique solution of problem (244) and the following stability inequalities hold:

$$
\begin{aligned}
& \max _{-T \leq t \leq T}\|u(t)\|_{H} \\
& \leq M\left[\|\varphi\|_{H}+\max _{-T \leq t \leq 0}\left\|A^{-1 / 2} g^{\prime}(t)\right\|_{H}+\left\|A^{-1 / 2} g(0)\right\|_{H}\right. \\
&\left.\quad+\left\|A^{-1 / 2} f(0)\right\|_{H}+\max _{0 \leq t \leq T}\left\|A^{-1 / 2} f^{\prime}(t)\right\|_{H}\right],
\end{aligned}
$$

$$
\begin{gathered}
\max _{-T \leq t \leq T}\left\|\frac{d u}{d t}\right\|_{H}+\max _{-T \leq t \leq T}\left\|A^{1 / 2} u(t)\right\|_{H} \\
\leq M\left[\left\|A^{1 / 2} \varphi\right\|_{H}+\|g(0)\|_{H}+\max _{-T \leq t \leq 0}\left\|g^{\prime}(t)\right\|_{H}\right. \\
\left.+\|f(0)\|_{H}+\max _{0 \leq t \leq T}\left\|f^{\prime}(t)\right\|_{H}\right], \\
\max _{-T \leq t \leq 0}\left\|\frac{d u}{d t}\right\|_{H}+\max _{0 \leq t \leq T}\left\|\frac{d^{2} u}{d t^{2}}\right\|_{H}+\max _{-T \leq t \leq T}\|A u(t)\|_{H} \\
\leq M\left[\|A \varphi\|_{H}+\left\|A^{1 / 2} g(0)\right\|_{H}+\left\|g^{\prime}(0)\right\|_{H}\right. \\
+\max _{-T \leq t \leq 0}\left\|g^{\prime \prime}(t)\right\|_{H}+\left\|A^{1 / 2} f(0)\right\|_{H} \\
\left.+\left\|f^{\prime}(0)\right\|_{H}+\max _{0 \leq t \leq T}\left\|f^{\prime \prime}(t)\right\|_{H}\right],
\end{gathered}
$$

where $M$ does not depend on $f(t), t \in[0, T], g(t) \in[-T, 0]$, or $\varphi$.

In [82], the first order of accuracy

$$
\begin{gathered}
\tau^{-2}\left(u_{k+1}-2 u_{k}+u_{k-1}\right)+A u_{k+1}=f_{k}, \\
f_{k}=f\left(t_{k+1}\right), \\
t_{k+1}=(k+1) \tau, \quad 1 \leq k<N, \\
\tau^{-1}\left(u_{k}-u_{k-1}\right)+A u_{k}=g_{k}, \\
g_{k}=g\left(t_{k}\right), \\
t_{k}=k \tau, \quad-N<k \leq 0, \\
\tau^{-1}\left(u_{1}-u_{0}\right)=-A u_{0}+g_{0}, \\
u_{-N}=\alpha u_{0}+\beta\left(-A u_{0}+g_{0}\right)+\varphi, \quad \mu \leq 2 \tau, \quad \lambda \leq 2 \tau, \\
u_{-N}=\alpha u_{[\mu / \tau]}+\beta\left(-A u_{0}+g_{0}\right)+\varphi, \quad 2 \tau<\mu, \lambda \leq 2 \tau, \\
u_{-N}=\alpha u_{0}+\beta \frac{u_{[\lambda / \tau]}-u_{[\lambda / \tau]-1}}{\tau}+\varphi, \quad \mu \leq 2 \tau, \quad 2 \tau<\lambda, \\
u_{-N}=\alpha u_{[\mu / \tau]}+\beta \frac{u_{[\lambda / \tau]}-u_{[\lambda / \tau]-1}}{\tau}+\varphi, \quad 2 \tau<\mu, 2 \tau<\lambda,
\end{gathered}
$$


and two types of second order of accuracy

$$
\begin{aligned}
& \tau^{-2}\left(u_{k+1}-2 u_{k}+u_{k-1}\right)+A u_{k}+\frac{\tau^{2}}{4} A^{2} u_{k+1}=f_{k}, \\
& f_{k}=f\left(t_{k}\right) \text {, } \\
& t_{k}=k \tau, \quad 1 \leq k<N, \\
& \tau^{-1}\left(I+\tau^{2} A\right)\left(u_{1}-u_{0}\right)=Z_{1}, \\
& Z_{1}=\frac{\tau}{2}\left(f(0)-A u_{0}\right)+\left(g(0)-A u_{0}\right), \\
& \tau^{-1}\left(u_{k}-u_{k-1}\right)+A\left(I+\frac{\tau}{2} A\right) u_{k}=\left(I+\frac{\tau}{2} A\right) g_{k} \\
& g_{k}=g\left(t_{k}-\frac{\tau}{2}\right) \\
& t_{k}=k \tau, \quad-(N-1) \leq k \leq 0, \\
& u_{-N}=\alpha\left(u_{0}+\mu\left(-A u_{0}+g_{0}\right)\right) \\
& +\beta\left(-A u_{0}+g_{0}+\lambda\left(-A u_{0}+f_{0}\right)\right) \\
& +\varphi, \quad \mu \leq 2 \tau, \lambda \leq 2 \tau, \\
& u_{-N}=\alpha\left(u_{[\mu / \tau]}+\left(\mu-\left[\frac{\mu}{\tau}\right] \tau\right) \frac{u_{[\mu / \tau]}-u_{[\mu / \tau]-1}}{\tau}\right) \\
& +\beta\left(-A u_{0}+g_{0}+\lambda\left(-A u_{0}+f_{0}\right)\right) \\
& +\varphi, \quad 2 \tau<\mu, \lambda \leq 2 \tau,
\end{aligned}
$$$$
u_{-N}=\alpha\left(u_{0}+\mu\left(-A u_{0}+g_{0}\right)\right)
$$$$
+\beta\left(\frac{u_{[\lambda / \tau]}-u_{[\lambda / \tau]-1}}{\tau}\right.
$$$$
\left.+\left(\lambda-\left[\frac{\lambda}{\tau}\right] \tau-\frac{\tau}{2}\right)\left(f_{[\lambda / \tau]}-A u_{[\lambda / \tau]}\right)\right)
$$

$+\varphi, \quad \mu \leq 2 \tau, 2 \tau<\lambda$,

$$
\begin{aligned}
u_{-N}= & \alpha\left(u_{[\mu / \tau]}+\left(\mu-\left[\frac{\mu}{\tau}\right] \tau\right) \frac{u_{[\mu / \tau]}-u_{[\mu / \tau]-1}}{\tau}\right) \\
& +\beta\left(\frac{u_{[\lambda / \tau]}-u_{[\lambda / \tau]-1}}{\tau}\right. \\
& \left.+\left(\lambda-\left[\frac{\lambda}{\tau}\right] \tau-\frac{\tau}{2}\right)\left(f_{[\lambda / \tau]}-A u_{[\lambda / \tau]}\right)\right)
\end{aligned}
$$$$
+\varphi, \quad 2 \tau<\mu, 2 \tau<\lambda
$$

$$
\begin{aligned}
& \tau^{-2}\left(u_{k+1}-2 u_{k}+u_{k-1}\right)+\frac{1}{2} A u_{k} \\
& +\frac{1}{4} A\left(u_{k+1}+u_{k-1}\right)=f_{k} \\
& f_{k}=f\left(t_{k}\right), \\
& t_{k}=k \tau, \quad 1 \leq k<N \text {, } \\
& \tau^{-1}\left(I+\tau^{2} A\right)\left(u_{1}-u_{0}\right)=Z_{1} \\
& Z_{1}=\frac{\tau}{2}\left(f(0)-A u_{0}\right)+\left(g(0)-A u_{0}\right), \\
& \tau^{-1}\left(u_{k}-u_{k-1}\right)+A\left(I+\frac{\tau}{2} A\right) u_{k}=\left(I+\frac{\tau}{2} A\right) g_{k} \\
& g_{k}=g\left(t_{k}-\frac{\tau}{2}\right) \\
& t_{k}=k \tau, \quad-(N-1) \leq k \leq 0, \\
& u_{-N}=\alpha\left(u_{0}+\mu\left(-A u_{0}+g_{0}\right)\right) \\
& +\beta\left(-A u_{0}+g_{0}+\lambda\left(-A u_{0}+f_{0}\right)\right) \\
& +\varphi, \quad \mu \leq 2 \tau, \lambda \leq 2 \tau, \\
& u_{-N}=\alpha\left(u_{[\mu / \tau]}+\left(\mu-\left[\frac{\mu}{\tau}\right] \tau\right) \frac{u_{[\mu / \tau]}-u_{[\mu / \tau]-1}}{\tau}\right) \\
& +\beta\left(-A u_{0}+g_{0}+\lambda\left(-A u_{0}+f_{0}\right)\right) \\
& +\varphi, \quad 2 \tau<\mu, \lambda \leq 2 \tau, \\
& u_{-N}=\alpha\left(u_{0}+\mu\left(-A u_{0}+g_{0}\right)\right) \\
& +\beta\left(\frac{u_{[\lambda / \tau]}-u_{[\lambda / \tau]-1}}{\tau}\right. \\
& \left.+\left(\lambda-\left[\frac{\lambda}{\tau}\right] \tau-\frac{\tau}{2}\right)\left(f_{[\lambda / \tau]}-A u_{[\lambda / \tau]}\right)\right) \\
& +\varphi, \quad \mu \leq 2 \tau, 2 \tau<\lambda \text {, } \\
& u_{-N}=\alpha\left(u_{[\mu / \tau]}+\left(\mu-\left[\frac{\mu}{\tau}\right] \tau\right) \frac{u_{[\mu / \tau]}-u_{[\mu / \tau]-1}}{\tau}\right) \\
& +\beta\left(\frac{u_{[\lambda / \tau]}-u_{[\lambda / \tau]-1}}{\tau}\right. \\
& \left.+\left(\lambda-\left[\frac{\lambda}{\tau}\right] \tau-\frac{\tau}{2}\right)\left(f_{[\lambda / \tau]}-A u_{[\lambda / \tau]}\right)\right) \\
& +\varphi, \quad 2 \tau<\mu, 2 \tau<\lambda,
\end{aligned}
$$

difference schemes for approximately solving boundary value problem (244) were presented. For the solution of these difference schemes, the following stability estimates are established. 
Theorem 104 (see [82]). Suppose that $\varphi \in D(A), g_{0} \in$ $D\left(A^{1 / 2}\right)$, and $f_{1} \in D\left(A^{1 / 2}\right)$. Then, for the solution of difference scheme (246), the following stability estimates hold:

$$
\begin{gathered}
\max _{-N \leq k \leq N}\left\|u_{k}\right\|_{H} \\
\leq M\left[\|\varphi\|_{H}+\left\|A^{-1 / 2} f_{1}\right\|_{H}+\left\|A^{-1 / 2} g_{0}\right\|_{H}\right. \\
+\max _{2 \leq k<N}\left\|A^{-1 / 2}\left(f_{k}-f_{k-1}\right) \tau^{-1}\right\|_{H} \\
\left.+\max _{-N<k \leq 0}\left\|A^{-1 / 2}\left(g_{k}-g_{k-1}\right) \tau^{-1}\right\|_{H}\right], \\
\max _{-N \leq k \leq N}\left\|A^{1 / 2} u_{k}\right\|_{H} \\
\leq M\left[\left\|A^{1 / 2} \varphi\right\|_{H}+\left\|f_{1}\right\|_{H}+\left\|g_{0}\right\|_{H}\right. \\
+\max _{2 \leq k \leq N-1}\left\|\left(f_{k}-f_{k-1}\right) \tau^{-1}\right\|_{H} \\
\left.+\max _{-(N-1) \leq k \leq 0}\left\|\left(g_{k}-g_{k-1}\right) \tau^{-1}\right\|_{H}\right], \\
\quad\left\|\tau^{-2}\left(u_{k+1}-2 u_{k}+u_{k-1}\right)\right\|_{H}+\max _{-N \leq k \leq N}\left\|A u_{k}\right\|_{H} \\
+\max _{-(N-1) \leq k \leq 0}\left\|\tau^{-1}\left(u_{k}-u_{k-1}\right)\right\|_{H} \\
+\left\|A_{-(N-1) \leq k \leq-1}^{1 / 2} g_{0}\right\|_{H}+\left\|\left(g_{0}-g_{-1}\right) \tau^{-1}\right\|_{H} \\
\left.+\left\|\left(g_{k+1}-2 g_{k}+g_{k-1}\right) \tau^{-2}\right\|_{H}\right], \\
+\max _{2 \leq k \leq N-2}\left\|\left(f_{k+1}-2 f_{k}+f_{k-1}\right) \tau^{-2}\right\|_{H} \\
+\|A \varphi\|_{H}+\left\|A^{1 / 2} f_{1}\right\|_{H} \\
+\left\|\tau^{-1}\right\|_{H}
\end{gathered}
$$

where $M$ does not depend on $\tau, f_{k}, 1 \leq k<N, g_{k},-N<k \leq$ 0 , or $\varphi$.

Theorem 105 (see [82]). Suppose that $\varphi \in D(A), g_{0} \in$ $D\left(A^{1 / 2}\right)$, and $f_{0} \in D\left(A^{1 / 2}\right)$. Then for the solution of difference schemes (247) and (248) the following stability estimates hold:

$$
\begin{aligned}
\max _{-N \leq k \leq N} \| & u_{k} \|_{H} \\
\leq M & {\left[\|\varphi\|_{H}+\left\|A^{-1 / 2} f_{0}\right\|_{H}+\left\|A^{-1 / 2} g_{0}\right\|_{H}\right.} \\
& +\max _{1 \leq k<N}\left\|\frac{A^{-1 / 2}}{\tau}\left(f_{k}-f_{k-1}\right)\right\|_{H} \\
& \left.+\max _{-N<k \leq 0}\left\|\frac{A^{-1 / 2}}{\tau}\left(g_{k}-g_{k-1}\right)\right\|_{H}\right],
\end{aligned}
$$

$$
\begin{gathered}
\max _{-N \leq k \leq N}\left\|A^{1 / 2} u_{k}\right\|_{H} \\
\leq M\left[\left\|A^{1 / 2} \varphi\right\|_{H}+\left\|f_{0}\right\|_{H}+\left\|g_{0}\right\|_{H}\right. \\
+\max _{1 \leq k \leq N-1}\left\|\left(f_{k}-f_{k-1}\right) \tau^{-1}\right\|_{H} \\
\left.+\max _{-(N-1) \leq k \leq 0}\left\|\left(g_{k}-g_{k-1}\right) \tau^{-1}\right\|_{H}\right], \\
+\max _{-(N-1) \leq k \leq 0}\left\|\tau^{-1}\left(u_{k}-u_{k-1}\right)\right\|_{H} \\
\quad \tau_{\left(u_{k+1}-2 u_{k}+u_{k-1}\right)\left\|_{H}+\max _{-N \leq k \leq N}\right\| A u_{k} \|_{H}}\left[\|A \varphi\|_{H}+\left\|A^{1 / 2} f_{0}\right\|_{H}+\left\|\left(f_{1}-f_{0}\right) \tau^{-1}\right\|_{H}\right. \\
+\max _{1 \leq k \leq N-2}\left\|\left(f_{k+1}-2 f_{k}+f_{k-1}\right) \tau^{-2}\right\|_{H} \\
+\left\|A_{-(N-1) \leq k \leq-1}^{1 / 2} g_{0}\right\|_{H}+\left\|\left(g_{0}-g_{-1}\right) \tau^{-1}\right\|_{H} \\
\left.\left.+\max _{k+1}-2 g_{k}+g_{k-1}\right) \tau^{-2} \|_{H}\right]
\end{gathered}
$$

where $M$ does not depend on $\tau, f_{k}, 0 \leq k<N, g_{k},-N<k \leq 0$, or $\varphi$.

In applications, the stability estimates for the solutions of the difference schemes of the mixed type boundary value problems for hyperbolic-parabolic equations were obtained. The theoretical statements for the solution of these difference schemes for hyperbolic-parabolic equation were supported by the results of numerical experiments.

The generalization of stability estimates results of $[82,83]$ was presented in $[84,85]$ for the solution of the multipoint nonlocal boundary value problem

$$
\begin{gathered}
\frac{d^{2} u(t)}{d t^{2}}+A u(t)=f(t) \quad(0 \leq t \leq T), \\
\frac{d u(t)}{d t}+A u(t)=g(t) \quad(-T \leq t \leq 0), \\
u(-T)=\sum_{i=1}^{N} \alpha_{i} u\left(\mu_{i}\right)+\sum_{i=1}^{L} \beta_{i} u^{\prime}\left(\lambda_{i}\right)+\varphi, \\
\sum_{i=1}^{N}\left|\alpha_{i}\right| \leq 1, \quad 0<\sum_{i=1}^{L}\left|\beta_{i}\right| \leq 1, \quad 0<\mu_{i}, \quad \lambda_{i} \leq T,
\end{gathered}
$$

for differential equations of mixed type in Hilbert space $H$ with self-adjoint positive definite operator $A$.

In practice, the stability estimates for the solutions of difference schemes of the nonlocal boundary value problems for one-dimensional hyperbolic-parabolic equations with nonlocal boundary conditions in space variable and multidimensional hyperbolic-parabolic equations with Dirichlet and 
Neumann conditions in space variables were obtained. The method was illustrated by numerical examples.

In $[90,91]$, the nonlocal boundary value problem

$$
\begin{array}{cc}
\frac{d^{2} u(t)}{d t^{2}}+A u(t)=f(t) & (0 \leq t \leq T), \\
i \frac{d u(t)}{d t}+A u(t)=g(t) & (-T \leq t \leq 0), \\
A u(-T)=\alpha u(\mu)+\varphi, & 0<\mu \leq T,
\end{array}
$$

for differential equations of hyperbolic-Schrödinger type in Hilbert space $H$ with self-adjoint positive definite operator $A$ was considered.

Theorem 106 (see [90]). Suppose that $\varphi \in D\left(A^{1 / 2}\right), f(0) \epsilon$ $D\left(A^{1 / 2}\right)$, and $g(0) \in D\left(A^{1 / 2}\right)$. Let $f(t)$ be continuously differentiable on $[0, T]$ and let $g(t)$ be twice continuously differentiable on $[-T, 0]$ functions. Then, there is a unique solution of problem (252) and the following stability inequalities

$$
\begin{aligned}
& \max _{-T \leq t \leq T}\|u(t)\|_{H} \\
& \leq M\left[\left\|A^{-1 / 2} \varphi\right\|_{H}+\left\|A^{-1 / 2} g(0)\right\|_{H}\right. \\
& +\max _{-T \leq t \leq 0}\left\|A^{-1} g^{\prime}(t)\right\|_{H} \\
& \left.+\max _{0 \leq t \leq T}\left\|A^{-1 / 2} f(t)\right\|_{H}\right] \text {, } \\
& \max _{-T \leq t \leq T}\left\|\frac{d u(t)}{d t}\right\|_{H}+\max _{-T \leq t \leq T}\left\|A^{1 / 2} u(t)\right\|_{H} \\
& \leq M\left[\|\varphi\|_{H}+\|g(0)\|_{H}+\max _{-T \leq t \leq 0}\left\|A^{-1 / 2} g^{\prime}(t)\right\|_{H}\right. \\
& \left.+\max _{0 \leq t \leq T}\|f(t)\|_{H}\right] \text {, } \\
& \max _{-T \leq t \leq 0}\left\|\frac{d u(t)}{d t}\right\|_{H}+\max _{0 \leq t \leq T}\left\|\frac{d^{2} u(t)}{d t^{2}}\right\|_{H}+\max _{-1 \leq t \leq 1}\|A u(t)\|_{H} \\
& \leq M\left[\left\|A^{1 / 2} \varphi\right\|_{H}+\left\|A^{1 / 2} g(0)\right\|_{H}+\left\|g^{\prime}(0)\right\|_{H}\right. \\
& +\max _{-T \leq t \leq 0}\left\|g^{\prime \prime}(t)\right\|_{H}+\left\|A^{1 / 2} f(0)\right\|_{H} \\
& \left.+\max _{0 \leq t \leq T}\left\|A^{1 / 2} f^{\prime}(t)\right\|_{H}\right]
\end{aligned}
$$

hold, where $M$ is independent of $f(t), t \in[0, T], g(t), t \in$ $[-T, 0]$, and $\varphi$.

In applications, the stability estimates for the solutions of the mixed type boundary value problems for hyperbolic-
Schrödinger equations were obtained. Difference schemes of first and second order of accuracy for approximate solutions of nonlocal boundary value problem (252) for hyperbolicSchrödinger equations were investigated. Stability estimates for the solutions of these difference schemes were obtained. A finite difference method and some results of numerical experiments are presented in order to support theoretical statements.

In [126], difference schemes for the approximate numerical solutions of difference schemes of multipoint nonlocal boundary value problem for the multidimensional hyperbolic-parabolic equations with Dirichlet and Neumann conditions were investigated. Stability estimates for the solution of these difference schemes and their first and second orders difference derivatives were obtained. Numerical experiments of one-dimensional hyperbolic-parabolic equations with variable conditions in $x$ and two-dimensional hyperbolic-parabolic equations were given. The theoretical statements for the solution of these difference schemes are supported by numerical examples.

Second, we consider the nonlocal boundary value problem

$$
\begin{gathered}
u_{t t}(t)+A u(t)=f(t), \quad 0 \leq t \leq T, \\
-u_{t t}(t)+A u(t)=g(t), \quad-T \leq t \leq 0, \\
u(-T)=u(T), \quad u(0)=\varphi
\end{gathered}
$$

for hyperbolic-elliptic differential equations in Hilbert space $H$, with the self-adjoint positive definite operator A.

Function $u(t)$ is called a solution of problem (254) if the following conditions are satisfied:

(i) $u(t)$ is twice continuously differentiable in the region $[-T, 0) \cup(0, T]$ and continuously differentiable on the segment $[-T, T]$.

(ii) The element $u(t)$ belongs to $D(A)$ for all $t \in[-T, T]$, and the function $A u(t)$ is continuous on $[-T, T]$.

(iii) $u(t)$ satisfies the equations and boundary conditions (254).

Theorem 107 (see [77]). Suppose that $\varphi \in D(A)$, and let $f(t)$ be continuously differentiable on $[0, T]$ and let $g(t)$ be continuously differentiable on $[-T, 0]$ functions. Then there is a unique solution of problem (254) and the stability inequalities

$$
\begin{aligned}
& \max _{-T \leq t \leq T}\|u(t)\|_{H} \\
& \leq M\left[\|\varphi\|_{H}+\max _{-T \leq t \leq 0}\left\|A^{-1 / 2} g(t)\right\|_{H}+\max _{0 \leq t \leq T}\left\|A^{-1 / 2} f(t)\right\|_{H}\right], \\
& \max _{-T \leq t \leq T}\left\|\frac{d u}{d t}\right\|_{H}+\max _{-T \leq t \leq T}\left\|A^{1 / 2} u(t)\right\|_{H}
\end{aligned}
$$




$$
\begin{gathered}
\leq M\left[\left\|A^{1 / 2} \varphi\right\|_{H}+\int_{-T}^{0}\|g(t)\|_{H} d t+\int_{0}^{T}\|f(t)\|_{H} d t\right], \\
\max _{-T \leq t \leq T}\left\|\frac{d^{2} u}{d t^{2}}\right\|_{H}+\max _{-T \leq t \leq T}\|A u(t)\|_{H} \\
\leq M\left[\|A \varphi\|_{H}+\|g(0)\|_{H}+\|f(0)\|_{H}\right. \\
\left.\quad+\int_{-T}^{0}\left\|g^{\prime}(t)\right\|_{H} d t+\int_{0}^{T}\left\|f^{\prime}(t)\right\|_{H} d t\right]
\end{gathered}
$$

hold, where $M$ does not depend on $f(t), t \in[0, T], g(t), t \in$ $[-T, 0]$, and $\varphi$.

In [77], applying the first order of accuracy difference scheme for hyperbolic equations and the second order of accuracy difference scheme for elliptic equations and the first order approximation formulae for nonlocal condition and continuity condition at $t=0$, the following first order of accuracy difference scheme

$$
\begin{gathered}
\frac{u_{k+1}-2 u_{k}+u_{k-1}}{\tau^{2}}+A u_{k+1}=f_{k}, \\
f_{k}=f\left(t_{k+1}\right), \\
t_{k+1}=(k+1) \tau, \quad 1 \leq k \leq N-1, \quad N \tau=T, \\
-\frac{u_{k+1}-2 u_{k}+u_{k-1}}{\tau^{2}}+A u_{k}=g_{k}, \\
g_{k}=g\left(t_{k}\right), \\
t_{k}=k \tau, \quad-N+1 \leq k \leq-1, \\
u_{-N}=u_{N}, \quad u_{0}=\varphi, \\
u_{1}-u_{0}=u_{0}-u_{-1},
\end{gathered}
$$

for approximately solving nonlocal boundary value problem (254), was presented.

Theorem 108 (see [77]). Let $\varphi \in D(A)$. Then, for the solution of difference scheme (256), the stability inequalities

$$
\begin{aligned}
& \max _{-N \leq t \leq N}\left\|u_{k}\right\|_{H} \\
& \quad \leq M\left[\|\varphi\|_{H^{+}} \max _{-N+1 \leq t \leq-1}\left\|A^{-1 / 2} g_{k}\right\|_{H}\right.
\end{aligned}
$$

$$
\begin{gathered}
\left.+\max _{1 \leq t \leq N-1}\left\|A^{-1 / 2} f_{k}\right\|_{H}\right], \\
\max _{-N+1 \leq t \leq N}\left\|\frac{u_{k}-u_{k-1}}{\tau}\right\|_{H}+\max _{-N \leq t \leq N}\left\|A^{1 / 2} u_{k}\right\|_{H} \\
\leq M\left[\left\|A^{1 / 2} \varphi\right\|_{H}+\sum_{k=-N+1}^{-1} \tau\left\|g_{k}\right\|_{H}+\sum_{k=1}^{N-1} \tau\left\|_{k}\right\|_{H}\right], \\
\max _{-N+1 \leq t \leq N-1} \| \frac{u_{k+1}-2 u_{k}+u_{k-1}\left\|_{H}+\max _{-N \leq t \leq N}\right\| A u_{k} \|_{H}}{\leq M}\left[\|A \varphi\|_{H}+\left\|g_{-1}\right\|_{H}+\left\|f_{1}\right\|_{H}\right. \\
\left.+\sum_{k=-N+1}^{-1}\left\|g_{k}-g_{k-1}\right\|_{H}+\sum_{k=2}^{N-1}\left\|f_{k}-f_{k-1}\right\|_{H}\right]
\end{gathered}
$$

hold, where $M$ does not depend on $\tau$, $\varphi$, or $f_{k}, 1 \leq k \leq N-$ $1, g_{k},-N+1 \leq k \leq-1$.

In [77], applying the second order of accuracy difference scheme for hyperbolic equations and elliptic equations and the second order approximation formulae for nonlocal condition and continuity condition at $t=0$, the following second order of accuracy difference schemes

$$
\begin{gathered}
\frac{u_{k+1}-2 u_{k}+u_{k-1}}{\tau^{2}}+A u_{k}+\frac{\tau^{2}}{4} A^{2} u_{k+1}=f_{k}, \\
f_{k}=f\left(t_{k}\right), \\
t_{k}=k \tau, \quad 1 \leq k \leq N-1, N \tau=T, \\
-\frac{u_{k+1}-2 u_{k}+u_{k-1}}{\tau^{2}}+A u_{k}=g_{k}, \\
g_{k}=g\left(t_{k}\right), \\
t_{k}=k \tau, \quad-N+1 \leq k \leq-1, \\
u_{1}-u_{0}-\frac{\tau^{2}}{2}\left(f_{0}-A u_{0}\right) \\
=u_{0}-u_{-1}-\frac{\tau^{2}}{2}\left(g_{0}-A u_{0}\right) \\
f_{0}=f(0), \quad g_{0}=g(0), \\
u_{0}=\varphi, \quad u_{-N}=u_{N},
\end{gathered}
$$




$$
\begin{gathered}
\frac{u_{k+1}-2 u_{k}+u_{k-1}}{\tau^{2}}+\frac{1}{2} A u_{k}+\frac{1}{4}\left(A u_{k+1}+A u_{k-1}\right)=f_{k}, \\
f_{k}=f\left(t_{k}\right), \\
t_{k}=k \tau, \quad 1 \leq k \leq N-1, N \tau=T, \\
-\frac{u_{k+1}-2 u_{k}+u_{k-1}}{\tau^{2}}+A u_{k}=g_{k}, \\
g_{k}=g\left(t_{k}\right), \\
\left(I+\frac{\tau^{2} A}{4}\right)\left(u_{1}-u_{0}\right)-\frac{\tau^{2}}{2}\left(f_{0}-A u_{0}\right) \\
=u_{0}-u_{-1}-\frac{\tau^{2}}{2}\left(g_{0}-A u_{0}\right), \\
f_{0}=f(0), \quad g_{0}=g(0), \\
u_{0}=\varphi, \quad u_{-N}=u_{N},
\end{gathered}
$$

for approximately solving nonlocal boundary value problem (254), was presented.

Theorem 109 (see [77]). Let $\varphi \in D(A)$. Then, for the solution of difference scheme (258), the stability inequalities

$$
\begin{aligned}
\max _{-N \leq t \leq N}\left\|u_{k}\right\|_{H} & \\
\leq M & {\left[\|\varphi\|_{H}+\max _{-N+1 \leq t \leq 0}\left\|A^{-1 / 2} g_{k}\right\|_{H}\right.} \\
& \left.+\max _{0 \leq t \leq N-1}\left\|A^{-1 / 2} f_{k}\right\|_{H}\right], \\
\max _{-N+1 \leq t \leq N}\left\|\frac{u_{k}-u_{k-1}}{\tau}\right\|_{H}+\max _{-N \leq t \leq N}\left\|A^{1 / 2} u_{k}\right\|_{H} & {\left[\left\|A^{1 / 2} \varphi\right\|_{H}+\sum_{k=-N+1}^{0} \tau\left\|g_{k}\right\|_{H}+\sum_{k=0}^{N-1} \tau\left\|f_{k}\right\|_{H}\right], } \\
\leq M & \max _{-N+1 \leq t \leq N-1}\left\|\frac{u_{k+1}-2 u_{k}+u_{k-1} \|}{\tau^{2}}\right\|_{H}+\max _{-N \leq t \leq N}\left\|A u_{k}\right\|_{H} \\
\leq M & {\left[\|A \varphi\|_{H}+\left\|g_{0}\right\|_{H}+\left\|f_{0}\right\|_{H}\right.} \\
& \left.+\sum_{k=-N+1}^{0}\left\|g_{k}-g_{k-1}\right\|_{H}+\sum_{k=1}^{N-1}\left\|f_{k}-f_{k-1}\right\|_{H}\right]
\end{aligned}
$$

hold, where $M$ does not depend on $\tau, \varphi$, or $f_{k}, 0 \leq k \leq N-$ $1, g_{k},-N+1 \leq k \leq 0$.
Theorem 110 (see [77]). Let $\varphi \in D\left(A^{3 / 2}\right)$. Then, for the solution of difference scheme (259), the stability inequalities

$$
\begin{aligned}
& \max _{-N \leq t \leq N} \| u_{k} \|_{H} \\
& \leq M\left\|\left(I \pm i \tau A^{1 / 2}\right) \varphi\right\|_{H} \\
&+\max _{-N+1 \leq t \leq 0}\left\|A^{-1 / 2} g_{k}\right\|_{H} \\
&\left.+\max _{0 \leq t \leq N-1}\left\|A^{-1 / 2} f_{k}\right\|_{H}\right], \\
& \max _{-N+1 \leq t \leq N} \| \frac{u_{k}-u_{k-1}\left\|_{H}+\max _{-N \leq t \leq N}\right\| A^{1 / 2} u_{k} \|_{H}}{\leq M}\left[\left\|A^{1 / 2}\left(I \pm i \tau A^{1 / 2}\right) \varphi\right\|_{H}\right. \\
&\left.+\sum_{k=-N+1}^{0} \tau\left\|g_{k}\right\|_{H}+\sum_{k=0}^{N-1} \tau\left\|f_{k}\right\|_{H}\right], \\
& \max _{-N+1 \leq t \leq N-1}\left\|\frac{u_{k+1}-2 u_{k}+u_{k-1}}{\tau \tau^{2}}\right\|_{H}+\max _{-N \leq t \leq N}\left\|A u_{k}\right\|_{H} \\
& \leq M\left[\left\|A\left(I \pm i \tau A^{1 / 2}\right) \varphi\right\|_{H}+\left\|g_{0}\right\|_{H}+\left\|f_{0}\right\|_{H}\right. \\
&\left.+\sum_{k=-N+1}^{0}\left\|g_{k}-g_{k-1}\right\|_{H}+\sum_{k=1}^{N-1}\left\|f_{k}-f_{k-1}\right\|_{H}\right]
\end{aligned}
$$

hold, where $M$ does not depend on $\tau, \varphi$, or $f_{k}, 0 \leq k \leq N-$ $1, g_{k},-N+1 \leq k \leq 0$.

The generalization of stability estimates results of $[77,126]$ was presented in [86] for the solution of the multipoint nonlocal boundary value problem

$$
\begin{gathered}
u_{t t}(t)+A u(t)=f(t), \quad 0 \leq t \leq T, \\
-u_{t t}(t)+A u(t)=g(t), \quad-T \leq t \leq 0, \\
u(-1)=\sum_{k=1}^{P} \alpha_{k} u\left(\theta_{k}\right)+\varphi, \\
0<\theta_{1}<\cdots<\theta_{P} \leq T, \\
u(0)=0
\end{gathered}
$$

for hyperbolic-elliptic differential equations of mixed type in Hilbert space $H$ with self-adjoint positive definite operator A.

In practice, the stability estimates for the solutions of difference schemes of the nonlocal boundary value problems for one-dimensional hyperbolic-elliptic equations with nonlocal boundary conditions in space variable and multidimensional hyperbolic-elliptic equations with Dirichlet and Neumann conditions in space variables were obtained. The method was illustrated by numerical examples. 
7.5. Stochastic Hyperbolic Equations. It is known that most problems for stochastic differential equations are applied to model diverse phenomena such as fluctuating stock prices or physical system subject to thermal fluctuations. Typically, stochastic differential equations incorporate white noise which can be thought of as the derivative of Brownian motion (or the Wiener process); however, it should be mentioned that other types of random fluctuations are possible, such as jump processes.

It is known that initial-boundary value problems for stochastic hyperbolic equations can be reduced to the Cauchy problem

$$
\begin{gathered}
v^{\prime \prime}(t) d t=-A v(t) d t+f(t) d w_{t}, \quad 0<t<T, \\
v(0)=0, \\
v^{\prime}(0)=0, \\
w_{t}=\sqrt{t} \xi, \quad \xi \in N(0,1),
\end{gathered}
$$

for the second order stochastic differential equation in Hilbert space $H$ with self-adjoint positive definite operator $A$ with $A \geq \delta I$, where $\delta>\delta_{0}>0$. Here,

(i) $w_{t}$ is a standard Wiener process given on the probability space $(\Omega, F, P)$;

(ii) for any $z \in[0, T], f(z)$ is an element of the space $M_{w}^{2}\left([0, T], H_{1}\right)$, where $H_{1}$ is a subspace of $H$.

Here, $M_{w}^{2}([0, T], H)$ denote the space of $H$-valued measurable processes which satisfy the following:

(a) $\phi(t)$ is $F_{t}$ measurable, a.e. in $t$.

(b) Consider $E \int_{0}^{T}\|\phi(t)\|_{H} d t<\infty$.

It is clear that under assumptions (i)-(ii) Cauchy problem (263) has a unique mild solution, which is represented by the following formula:

$$
v(t)=\int_{0}^{t} S(t-z, A) f(z) d w_{z} .
$$

Theorem 111 (see [93]). Let $v\left(t_{k}\right)$ be the solution of (263) at the grid points $t=t_{k}$. Then $\left\{v\left(t_{k}\right)\right\}_{0}^{N}$ is the solution of the initial value problem for the following difference equation:

$$
\begin{gathered}
\frac{1}{\tau^{2}}\left(v\left(t_{k+1}\right)-2 v\left(t_{k}\right)+v\left(t_{k-1}\right)\right) \\
+\frac{2}{\tau^{2}}(I-C(\tau, A)) v\left(t_{k}\right)=f_{k}, \\
f_{k}=\frac{1}{\tau}\left(f_{1, k+1}+S(\tau, A) f_{2, k}-C(\tau, A) f_{1, k}\right), \\
f_{1, k}=\frac{1}{\tau} \int_{t_{k-1}}^{t_{k}} S\left(t_{k}-z, A\right) f(z) d w_{z}, \\
f_{2, k}=\frac{1}{\tau} \int_{t_{k-1}}^{t_{k}} C\left(t_{k}-z, A\right) f(z) d w_{z}, \\
v(0)=0, \quad v(\tau)=\tau f_{1,1} .
\end{gathered}
$$

For the approximate solution of problem (263), we need to approximate the expressions

$$
\begin{gathered}
f_{1, k}=\frac{1}{\tau} \int_{t_{k-1}}^{t_{k}} S\left(t_{k}-z, A\right) f(z) d w_{z}, \\
f_{2, k}=\frac{1}{\tau} \int_{t_{k-1}}^{t_{k}} C\left(t_{k}-z, A\right) f(z) d w_{z}, \\
\exp \left( \pm i \tau A^{1 / 2}\right) .
\end{gathered}
$$

Using Taylor's formula and Padé approximation of the function $e^{-z}$ at $z=0$, we can construct the following two-step difference scheme:

$$
\begin{gathered}
\frac{1}{\tau^{2}}\left(u_{k+1}-2 u_{k}+u_{k-1}\right)+\frac{1}{2} A u_{k}+\frac{1}{4} A u_{k+1}+A u_{k-1} \\
=\frac{1}{\tau}\left(\left(I+\frac{1}{4} \tau^{2} A\right) \tilde{f}_{1, k+1}+\tau \tilde{f}_{2, k}-\left(I-\frac{1}{4} \tau^{2} A\right) \tilde{f}_{1, k}\right), \\
t_{k}=k \tau, \quad 1 \leq k \leq N-1, \\
u_{0}=0, \quad u_{1}=\tau \tilde{f}_{1,1},
\end{gathered}
$$

for the approximate solution of problem (263).

Theorem 112 (see [93]). Assume that

$$
E \int_{0}^{T}\|A f(t)\|_{H}^{2} d t \leq C .
$$

Then the estimate of convergence

$$
\left(\sum_{k=1}^{N} E\left\|v\left(t_{k}\right)-u_{k}\right\|_{H}^{2}\right)^{1 / 2} \leq C_{1}(\delta) \tau
$$

holds. Here, $C_{1}(\delta)$ does not depend on $\tau$.

In applications, the initial-boundary value problem for one-dimensional stochastic hyperbolic equation

$$
\begin{gathered}
u_{t t}(t, x) d t-\left(a(x) u_{x}\right)_{x} d t+\delta u(t, x) d t=f(t, x) d w_{t}, \\
0<t<T, \quad 0<x<1, \\
u(0, x)=u_{t}(0, x)=0, \quad 0 \leq x \leq 1, \\
u(t, 0)=u(t, 1), \quad u_{x}(t, 0)=u_{x}(t, 1), \quad 0 \leq t \leq T,
\end{gathered}
$$

was considered. Here $\delta>0, a(x) \geq a>0, a(1)=$ $a(0)(x \in(0,1))$, and $f(t, x)(t \in[0, T], x \in[0,1])$ are smooth functions with respect to $x$.

The discretization of problem (270) is carried out in two steps. In the first step, we define the grid space

$$
[0,1]_{h}=\left\{x=x_{n}: x_{n}=n h, 0 \leq n \leq M, M h=1\right\} .
$$

Let us introduce the Hilbert space $L_{2 h}=L_{2}\left([0,1]_{h}\right)$ of the grid functions $\varphi^{h}(x)=\left\{\varphi_{n}\right\}_{1}^{M-1}$ defined on $[0,1]_{h}$, equipped with the norm

$$
\left\|\varphi^{h}\right\|_{L_{2 h}}=\left(\sum_{x \in[0,1]_{h}}|\varphi(x)|^{2} h\right)^{1 / 2} .
$$


To the differential operator $A$ generated by problem (270), we assign the difference operator $A_{h}^{x}$ by the formula

$$
A_{h}^{x} \varphi^{h}(x)=\left\{-\left(a(x) \varphi_{\bar{x}}\right)_{x, n}+\delta \varphi_{n}\right\}_{1}^{M-1}
$$

acting in the space of grid functions $\varphi^{h}(x)=\left\{\varphi_{n}\right\}_{0}^{M}$ satisfying the conditions $\varphi_{0}=\varphi_{M}, \varphi_{1}-\varphi_{0}=\varphi_{M}-\varphi_{M-1}$. It is wellknown that $A_{h}^{x}$ is a self-adjoint positive definite operator in $L_{2 h}$. With the help of $A_{h}^{x}$, we arrive at the following initial value problem:

$$
\begin{array}{r}
u_{t t}^{h}(t, x) d t+A_{h}^{x} u^{h}(t, x) d t=f^{h}(t, x) d w_{t}, \\
0<t<T, \quad x \in[0,1]_{h}, \\
u^{h}(0, x)=u_{t}^{h}(0, x)=0, \quad x \in[0,1]_{h} .
\end{array}
$$

In the second step, we replace (274) with difference scheme (267):

$$
\begin{gathered}
\frac{1}{\tau^{2}}\left(u_{k+1}^{h}(x)-2 u_{k}^{h}(x)+u_{k-1}^{h}(x)\right)+\frac{1}{2} A_{h}^{x} u_{k}^{h}(x) \\
+\frac{1}{4}\left(A_{h}^{x} u_{k+1}^{h}(x)+A_{h}^{x} u_{k-1}^{h}(x)\right)=\varphi_{k}^{h}(x), \\
\varphi_{k}^{h}(x)=\frac{1}{\tau}\left(\left(I+\frac{1}{4} \tau^{2} A_{h}^{x}\right) \varphi_{1, k+1}^{h}(x)+\tau \varphi_{2, k}^{h}(x)\right. \\
\left.-\left(I-\frac{1}{4} \tau^{2} A_{h}^{x}\right) \varphi_{1, k}^{h}(x)\right), \\
\varphi_{1, k}^{h}(x)=-\frac{1}{\tau} \int_{t_{k-1}}^{t_{k}}\left(z-t_{k}\right) f^{h}(z, x) d w_{z}, \\
\varphi_{2, k}^{h}(x)=\frac{1}{\tau} \int_{t_{k-1}}^{t_{k}} f(z) d w_{z}, \\
t_{k}=k \tau, \quad 1 \leq k \leq N-1, x \in[0,1]_{h}, \\
u_{0}^{h}(x)=0, \quad u_{1}^{h}(x)=-\int_{0}^{\tau}(z-\tau) f^{h}(z, x) d w_{z}, \\
x \in[0,1]_{h} .
\end{gathered}
$$

Theorem 113 (see [93]). Let $\tau$ and $h$ be sufficiently small numbers. Then, the solutions of difference scheme (275) satisfy the following convergence estimate:

$$
\left(\sum_{k=1}^{N} E\left\|u^{h}\left(t_{k}\right)-u_{k}^{h}\right\|_{L_{2 h}}^{2}\right)^{1 / 2} \leq C(\delta)(\tau+h),
$$

where $C(\delta)$ does not depend on $\tau$ or $h$.

The proof of Theorem 113 is based on the abstract Theorem 112 and the symmetry properties of the difference operator $A_{h}^{x}$ defined by formula (273).

Second, let $\Omega$ be the unit open cube in the $n$-dimensional Euclidean space $\mathbb{R}^{n}=\left\{x=\left(x_{1}, \ldots, x_{n}\right): 0<x_{i}<1, i=\right.$ $1, \ldots, n\}$ with boundary $S, \bar{\Omega}=\Omega \cup S$. In $[0, T] \times \Omega$, the mixed boundary value problem for the multidimensional parabolic equation

$$
\begin{gathered}
u_{t t}(t, x) d t-\sum_{r=1}^{n}\left(a_{r}(x) u_{x_{r}}\right)_{x_{r}} d t=f(t, x) d w_{t}, \\
0<t<T, \quad x=\left(x_{1}, \ldots, x_{n}\right) \in \Omega, \\
u(0, x)=u_{t}(0, x)=0, \quad x \in \bar{\Omega} ; \\
u(t, x)=0, \quad x \in S, \quad 0 \leq t \leq T,
\end{gathered}
$$

with the Dirichlet condition, was considered. Here $a_{r}(x),(x \in$ $\Omega)$, and $f(t, x)(t \in(0,1), x \in \Omega)$ are given smooth functions with respect to $x$ and $a_{r}(x) \geq a>0$.

The discretization of problem (277) is carried out in two steps. In the first step, define the grid space $\bar{\Omega}_{h}=\left\{x=x_{m}=\right.$ $\left(h_{1} m_{1}, \ldots, h_{n} m_{n}\right) ; m=\left(m_{1}, \ldots, m_{n}\right), 0 \leq m_{r} \leq N_{r}, h_{r} N_{r}=$ $1, r=1, \ldots, n\}, \Omega_{h}=\bar{\Omega}_{h} \cap \Omega, S_{h}=\bar{\Omega}_{h} \cap S$.

Let $L_{2 h}$ denote the Hilbert space

$$
\begin{aligned}
L_{2 h} & =L_{2}\left(\bar{\Omega}_{h}\right) \\
& =\left\{\varphi^{h}(x):\left(\sum_{x \in \bar{\Omega}_{h}}\left|\varphi^{h}(x)\right|^{2} h_{1} \cdots h_{n}\right)^{1 / 2}<\infty\right\} .
\end{aligned}
$$

The differential operator $A$ in (277) is replaced with

$$
A_{h}^{x} u^{h}(x)=-\sum_{r=1}^{n}\left(a_{r}(x) u_{\bar{x}_{r}}^{h}\right)_{x_{r}, j_{r}},
$$

where the difference operator $A_{h}^{x}$ is defined on those grid functions $u^{h}(x)=0$, for all $x \in S_{h}$. It is well-known that $A_{h}^{x}$ is a self-adjoint positive definite operator in $L_{2 h}$.

Using (279), we arrive at the following initial value problem:

$$
\begin{gathered}
u_{t t}^{h}(t, x) d t+A_{h}^{x} u^{h}(t, x) d t=f^{h}(t, x) d w_{t}, \\
0<t<T, \quad x \in \Omega_{h}, \\
u^{h}(0, x)=u_{t}^{h}(0, x)=0, \quad x \in \bar{\Omega}_{h} .
\end{gathered}
$$


In the second step, we replace (280) with difference scheme (267):

$$
\begin{gathered}
\frac{1}{\tau^{2}}\left(u_{k+1}^{h}(x)-2 u_{k}^{h}(x)+u_{k-1}^{h}(x)\right)+\frac{1}{2} A_{h}^{x} u_{k}^{h}(x) \\
+\frac{1}{4}\left(A_{h}^{x} u_{k+1}^{h}(x)+A_{h}^{x} u_{k-1}^{h}(x)\right)=\varphi_{k}^{h}(x), \\
\varphi_{k}^{h}(x)=\frac{1}{\tau}\left(\left(I+\frac{1}{4} \tau^{2} A_{h}^{x}\right) \varphi_{1, k+1}^{h}(x)+\tau \varphi_{2, k}^{h}(x)\right. \\
\left.-\left(I-\frac{1}{4} \tau^{2} A_{h}^{x}\right) \varphi_{1, k}^{h}(x)\right), \\
\varphi_{1, k}^{h}(x)=-\frac{1}{\tau} \int_{t_{k-1}}^{t_{k}}\left(z-t_{k}\right) f^{h}(z, x) d w_{z}, \\
\varphi_{2, k}^{h}(x)=\frac{1}{\tau} \int_{t_{k-1}}^{t_{k}} f(z) d w_{z}, \\
t_{k}=k \tau, \quad 1 \leq k \leq N-1, x \in \Omega_{h}, \\
u_{0}^{h}(x)=0, \quad x \in \Omega_{h}^{h} .
\end{gathered}
$$

Theorem 114 (see [93]). Let $\tau$ and $|h|=\sqrt{h_{1}^{2}+\cdots+h_{n}^{2}}$ be sufficiently small numbers. Then, the solution of difference scheme (281) satisfies the following convergence estimate:

$$
\left(\sum_{k=1}^{N} E\left\|u^{h}\left(t_{k}\right)-u_{k}^{h}\right\|_{L_{2 h}}^{2}\right)^{1 / 2} \leq C(\delta)\left(\tau+|h|^{2}\right),
$$

where $C(\delta)$ does not depend on $\tau$ or $|h|$.

The proof of Theorem 114 is based on the abstract Theorem 112 and the symmetry properties of the difference operator $A_{h}^{x}$ defined by formula (279).

Third, in $[0, T] \times \Omega$, the mixed boundary value problem for the multidimensional parabolic equation

$$
\begin{array}{r}
u_{t t}(t, x) d t-\sum_{r=1}^{n}\left(a_{r}(x) u_{x_{r}}\right)_{x_{r}} d t \\
+\delta u(t, x) d t=f(t, x) d w_{t}, \\
0<t<T, \quad x=\left(x_{1}, \ldots, x_{n}\right) \in \Omega, \\
u(0, x)=u_{t}(0, x)=0, \quad x \in \bar{\Omega} ; \\
\frac{\partial u(t, x)}{\partial \vec{n}}=0, \quad x \in S, \quad 0 \leq t \leq T,
\end{array}
$$

with the Neumann condition, was considered. Here $\vec{n}$ is the normal vector to $\Omega$. Here $\delta>0 a_{r}(x),(x \in \Omega)$, and $f(t, x)(t \in(0,1), x \in \Omega)$ are given smooth functions with respect to $x$ and $a_{r}(x) \geq a>0$.
The discretization of problem (283) is carried out in two steps. In the first step, the differential operator $A$ in (283) is replaced with

$$
A_{h}^{x} u^{h}(x)=-\sum_{r=1}^{n}\left(a_{r}(x) u_{\bar{x}_{r}}^{h}\right)_{x_{r}, j_{r}}+\delta u^{h}(x)
$$

where the difference operator $A_{h}^{x}$ is defined on those grid functions $D^{h} u^{h}(x)=0$, for all $x \in S_{h}$, where $D^{h} u^{h}(x)=0$ is the second order of approximation of $\partial u(t, x) / \partial \vec{n}$. It is easy to see that $A_{h}^{x}$ is a self-adjoint positive definite operator in $L_{2 h}$. Using (284), we arrive at the following initial value problem:

$$
\begin{array}{r}
u_{t t}^{h}(t, x) d t+A_{h}^{x} u^{h}(t, x) d t=f^{h}(t, x) d w_{t}, \\
0<t<T, \quad x \in \Omega_{h}, \\
u^{h}(0, x)=u_{t}^{h}(0, x)=0, \quad x \in \bar{\Omega}_{h} .
\end{array}
$$

In the second step, we replace (285) with the difference scheme (267):

$$
\begin{gathered}
\frac{1}{\tau^{2}}\left(u_{k+1}^{h}(x)-2 u_{k}^{h}(x)+u_{k-1}^{h}(x)\right)+\frac{1}{2} A_{h}^{x} u_{k}^{h}(x) \\
+\frac{1}{4}\left(A_{h}^{x} u_{k+1}^{h}(x)+A_{h}^{x} u_{k-1}^{h}(x)\right)=\varphi_{k}^{h}(x), \\
\varphi_{k}^{h}(x)=\frac{1}{\tau}\left(\left(I+\frac{1}{4} \tau^{2} A_{h}^{x}\right) \varphi_{1, k+1}^{h}(x)+\tau \varphi_{2, k}^{h}(x)\right. \\
\left.-\left(I-\frac{1}{4} \tau^{2} A_{h}^{x}\right) \varphi_{1, k}^{h}(x)\right), \\
\varphi_{1, k}^{h}(x)=-\frac{1}{\tau} \int_{t_{k-1}}^{t_{k}}\left(z-t_{k}\right) f^{h}(z, x) d w_{z}, \\
\varphi_{2, k}^{h}(x)=\frac{1}{\tau} \int_{t_{k-1}}^{t_{k}} f(z) d w_{z}, \\
t_{k}=k \tau, \quad 1 \leq k \leq N-1, x \in \Omega_{h}, \\
u_{0}^{h}(x)=0, \quad u_{1}^{h}(x)=-\int_{0}^{\tau}(z-\tau) f^{h}(z, x) d w_{z},
\end{gathered}
$$

$$
x \in \Omega_{h} .
$$

Theorem 115 (see [93]). Let $\tau$ and $|h|=\sqrt{h_{1}^{2}+\cdots+h_{n}^{2}}$ be sufficiently small numbers. Then, the solution of difference scheme (286) satisfies the following convergence estimate:

$$
\left(\sum_{k=1}^{N} E\left\|u^{h}\left(t_{k}\right)-u_{k}^{h}\right\|_{L_{2 h}}^{2}\right)^{1 / 2} \leq C(\delta)\left(\tau+|h|^{2}\right),
$$

where $C(\delta)$ does not depend on $\tau$ or $|h|$.

The proof of Theorem 115 is based on the abstract Theorem 112 and the symmetry properties of the difference operator $A_{h}^{x}$ defined by formula (284). 
Fourth, in $[0, T] \times \Omega$, the mixed boundary value problem for the multidimensional parabolic equation

$$
\begin{gathered}
u_{t t}(t, x) d t-\sum_{r=1}^{n}\left(a_{r}(x) u_{x_{r}}\right)_{x_{r}} d t \\
+\delta u(t, x) d t=f(t, x) d w_{t}, \\
0<t<T, \quad x=\left(x_{1}, \ldots, x_{n}\right) \in \Omega, \\
u(0, x)=u_{t}(0, x)=0, \quad x \in \bar{\Omega} ; \\
\frac{\partial u(t, x)}{\partial \vec{n}}=0, \quad x \in S_{2}, \\
0 \leq t \leq T, \quad S_{1} \cup S_{2}=S, \\
u(t, x)=0, \quad x \in S_{1},
\end{gathered}
$$

with the Dirichlet and Neumann conditions was considered. Here $\vec{n}$ is the normal vector to $\Omega$. Here $\delta>0 a_{r}(x),(x \in \Omega)$, and $f(t, x)(t \in(0,1), x \in \Omega)$ are given smooth functions with respect to $x$ and $a_{r}(x) \geq a>0$.

The discretization of problem (288) is carried out in two steps. In the first step, the differential operator $A$ in (288) is replaced with

$$
A_{h}^{x} u^{h}(x)=-\sum_{r=1}^{n}\left(a_{r}(x) u_{\bar{x}_{r}}^{h}\right)_{x_{r}, j_{r}}+\delta u^{h}(x),
$$

where the difference operator $A_{h}^{x}$ is defined on those grid functions $u^{h}(x)=0$, for all $x \in S_{h}^{1}$ and $D^{h} u^{h}(x)=0$, for all $x \in S_{h}^{2}, S_{h}^{1} \cup S_{h}^{2}=S_{h}$, where $D^{h} u^{h}(x)=0$ is the second order of approximation of $\partial u(t, x) / \partial \vec{n}$. By [101], we can conclude that $A_{h}^{x}$ is a self-adjoint positive definite operator in $L_{2 h}$. Using (289), we arrive at initial value problem (285).

In the second step, we replace (285) with difference scheme (267):

$$
\begin{gathered}
\frac{1}{\tau^{2}}\left(u_{k+1}^{h}(x)-2 u_{k}^{h}(x)+u_{k-1}^{h}(x)\right)+\frac{1}{2} A_{h}^{x} u_{k}^{h}(x) \\
+\frac{1}{4}\left(A_{h}^{x} u_{k+1}^{h}(x)+A_{h}^{x} u_{k-1}^{h}(x)\right)=\varphi_{k}^{h}(x), \\
\varphi_{k}^{h}(x)=\frac{1}{\tau}\left(\left(I+\frac{1}{4} \tau^{2} A_{h}^{x}\right) \varphi_{1, k+1}^{h}(x)+\tau \varphi_{2, k}^{h}(x)\right. \\
\left.-\left(I-\frac{1}{4} \tau^{2} A_{h}^{x}\right) \varphi_{1, k}^{h}(x)\right), \\
\varphi_{1, k}^{h}(x)=-\frac{1}{\tau} \int_{t_{k-1}}^{t_{k}}\left(z-t_{k}\right) f^{h}(z, x) d w_{z}, \\
\varphi_{2, k}^{h}(x)=\frac{1}{\tau} \int_{t_{k-1}}^{t_{k}} f(z) d w_{z}, \\
t_{k}=k \tau, \quad 1 \leq k \leq N-1, N \tau=T, x \in \Omega_{h}, \\
u_{0}^{h}(x)=0, \quad u_{1}^{h}(x)=-\int_{0}^{\tau}(z-\tau) f^{h}(z, x) d w_{z}, \\
x \in \Omega_{h} .
\end{gathered}
$$

Theorem 116 (see [93]). Let $\tau$ and $|h|=\sqrt{h_{1}^{2}+\cdots+h_{n}^{2}}$ be sufficiently small numbers. Then, the solution of difference scheme (290) satisfies the following convergence estimate:

$$
\left(\sum_{k=1}^{N} E\left\|u^{h}\left(t_{k}\right)-u_{k}^{h}\right\|_{L_{2 h}}^{2}\right)^{1 / 2} \leq C(\delta)\left(\tau+|h|^{2}\right),
$$

where $C(\delta)$ does not depend on $\tau$ or $|h|$.

The proof of Theorem 116 is based on abstract Theorem 112 and the symmetry properties of the difference operator $A_{h}^{x}$ defined by formula (284).

In [92], the initial value problem

$$
\begin{gathered}
d \dot{v}(t)+A v(t) d t=f(t) d w_{t}, \quad 0<t<T, \\
v(0)=\varphi, \quad \dot{v}(0)=\psi,
\end{gathered}
$$

for a stochastic hyperbolic equation in Hilbert space $H$ with self-adjoint positive definite operator $A$ with $A \geq \delta I$, where $\delta>\delta_{0}>0$, was investigated. In addition to (i) and (ii), we put the following:

(iii) $\varphi$ and $\psi$ are elements of the space $M_{w}^{2}\left([0, T], H_{2}\right)$ of $\mathrm{H}_{2}$-valued measurable processes, where $\mathrm{H}_{2}$ is a subspace of $H$.

Then, under assumptions (i), (ii), and (iii), initial value problem (292) has a unique mild solution given by the formula

$$
v(t)=C(t, A) \varphi+S(t, A) \psi+\int_{0}^{t} S(t-z, A) f(z) d w_{z} .
$$

Applying the method of [93] and formula (293), the difference scheme for the approximate solution of initial value problem (292) was constructed and investigated. The convergence estimate for the solution of the difference scheme was proved. In applications, the theorems on convergence estimates for the solution of difference schemes for the approximate solution of initial-boundary value problems for hyperbolic equations with Neumann, Dirichlet, DirichletNeumann, and Neumann-Dirichlet conditions were proved. Thus, results show that the error is stable and decreases in an exponential manner.

7.6. Fractional Hyperbolic Equations. In [96], the initial value problem

$$
\begin{gathered}
\frac{d^{2} v(t)}{d t^{2}}+D_{t}^{1 / 2} v(t)+A v(t)=f(t), \quad 0<t<T, \\
v(0)=0, \quad v^{\prime}(0)=\psi
\end{gathered}
$$

for the fractional differential equation of the hyperbolic type in Hilbert space $H$ with self-adjoint positive definite operator $A$ with $A \geq \delta I$, where $\delta>\delta_{0}>0$ was considered. 
Function $v(t)$ is called a solution of problem (294) if the following conditions are satisfied:

(i) $v(t)$ is twice continuously differentiable on the segment $[0, T]$.

(ii) The element $v(t)$ belongs to $D(A)$ for all $t \in[0, T]$ and the function $A v(t)$ is continuous on the segment $[0, T]$.

(iii) $u(t)$ satisfies the equation and initial conditions (294).

Theorem 117 (see [96]). Suppose that $\psi \in H$ and let $f(t)$ be a continuous function defined on $[0, T]$. Then, the following stability estimates hold:

$$
\begin{aligned}
\max _{0 \leq t \leq T} & \|v(t)\|_{H}+\max _{0 \leq t \leq T}\left\|v_{t}\right\|_{H} \\
& +\max _{0 \leq t \leq T}\left\|D_{t}^{1 / 2} v(t)\right\|_{H}+\max _{0 \leq t \leq T}\left\|v_{t t}+v(t)\right\|_{H} \\
\leq & M\left[\max _{0 \leq t \leq T}\|f(t)\|_{H}+\|\psi\|_{H}\right] .
\end{aligned}
$$

Suppose that $\psi=0$ and $f(t)$ is a continuously differentiable function defined $[0, T]$. Then, the following stability estimates hold:

$$
\begin{aligned}
& \max _{0 \leq t \leq T}\left\|\frac{d^{2} v(t)}{d t^{2}}\right\|_{H}+\max _{0 \leq t \leq T}\|A v(t)\|_{H}+\max _{0 \leq t \leq T}\left\|D_{t}^{3 / 2} v(t)\right\|_{H} \\
& \quad \leq M\left[\|f(0)\|_{H}+\max _{0 \leq t \leq T}\left\|f^{\prime}(t)\right\|_{H}\right] .
\end{aligned}
$$

Here $M$ does not depend on $\psi$ or $f(t), t \in[0, T]$.

In applications, the stability estimates for the solution of two problems were established. First, the mixed problem for the fractional hyperbolic equation

$$
\begin{gathered}
u_{t t}+D_{t}^{1 / 2} u(t)-\left(a(x) u_{x}\right)_{x}+\delta u=f(t, x), \\
0<t<T, \quad 0<x<1, \\
u(0, x)=0, \quad u_{t}(0, x)=0, \quad 0 \leq x \leq 1, \\
u(t, 0)=u(t, 1), \quad u_{x}(t, 0)=u_{x}(t, 1), \quad 0 \leq t \leq T,
\end{gathered}
$$

was considered. Problem (297) has unique smooth solution $u(t, x)$ for $\delta>0$ and the smooth functions $a(x) \geq a>0$, $a(0)=a(1)(x \in(0,1))$, and $f(t, x)(t \in[0, T], x \in[0,1])$. This allows us to reduce mixed problem (297) to initialboundary value problem (294) in Hilbert space $H=L_{2}[0,1]$ with self-adjoint positive definite operator $A^{x}$ defined by formula (297).
Theorem 118 (see [96]). For solutions of mixed problem (297), one has the following stability inequalities:

$$
\begin{aligned}
& \max _{0 \leq t \leq T}\|u(t, \cdot)\|_{L_{2}[0,1]}+\max _{0 \leq t \leq T}\left\|u_{t}(t, \cdot)\right\|_{L_{2}[0,1]} \\
& \quad \leq M \max _{0 \leq t \leq T}\|f(t, \cdot)\|_{L_{2}[0,1]}, \\
& \max _{0 \leq t \leq T}\left\|u_{x x}(t, \cdot)\right\|_{L_{2}[0,1]}+\max _{0 \leq t \leq T}\left\|u_{t t}(t, \cdot)\right\|_{L_{2}[0,1]} \\
& \quad \leq M\left[\max _{0 \leq t \leq T}\left\|f_{t}(t, \cdot)\right\|_{L_{2}[0,1]}+\|f(0, \cdot)\|_{L_{2}[0,1]}\right],
\end{aligned}
$$

where $M$ does not depend on $f(t, x)$.

The proof of Theorem 118 is based on abstract Theorem 117 and the symmetry properties of the operator $A^{x}$ defined by formula (297).

Second, let $\Omega$ be the unit open cube in the $m$-dimensional Euclidean space $\mathbb{R}^{m}\left\{x=\left(x_{1}, \ldots, x_{m}\right): 0<x_{j}<1,1 \leq j \leq\right.$ $m$ \} with boundary $S, \bar{\Omega}=\Omega \cup S$. In $[0, T] \times \Omega$, the mixed boundary value problem for the multidimensional fractional hyperbolic equation

$$
\begin{gathered}
\frac{\partial^{2} u(t, x)}{\partial t^{2}}-\sum_{r=1}^{m}\left(a_{r}(x) u_{x_{r}}\right)_{x_{r}}+D_{t}^{1 / 2} u(t, x)=f(t, x), \\
x=\left(x_{1}, \ldots, x_{m}\right) \in \Omega, \quad 0<t<T, \\
u(0, x)=0, \quad u_{t}(0, x)=0, \quad x \in \bar{\Omega} ; \\
u(t, x)=0, \quad x \in S,
\end{gathered}
$$

was considered. Here $a_{r}(x),(x \in \Omega)$, and $f(t, x)(t \in$ $(0, T), x \in \Omega)$ are given smooth functions and $a_{r}(x) \geq a>0$.

Problem (299) has a unique smooth solution $u(t, x)$ for the smooth functions $a_{r}(x)$ and $f(t, x)$. This allows us to reduce mixed problem (299) to initial-boundary value problem (294) in Hilbert space $H=L_{2}(\bar{\Omega})$ with self-adjoint positive definite operator $A^{x}$ defined by formula (299).

Theorem 119 (see [96]). For the solutions of mixed problem (299), the following stability inequalities

$$
\begin{aligned}
& \max _{0 \leq t \leq T}\|u(t, \cdot)\|_{L_{2}(\bar{\Omega})}+\max _{0 \leq t \leq T}\left\|u_{t}(t, \cdot)\right\|_{L_{2}(\bar{\Omega})} \\
& \quad \leq M \max _{0 \leq t \leq T}\|f(t, \cdot)\|_{L_{2}(\bar{\Omega})}, \\
& \max _{0 \leq t \leq T} \sum_{r=1}^{m}\left\|u_{x_{r} x_{r}}(t, \cdot)\right\|_{L_{2}(\bar{\Omega})}+\max _{0 \leq t \leq T}\left\|u_{t t}(t, \cdot)\right\|_{L_{2}(\bar{\Omega})} \\
& \quad \leq M\left[\max _{0 \leq t \leq T}\left\|f_{t}(t, \cdot)\right\|_{L_{2}(\bar{\Omega})}+\|f(0, \cdot)\|_{L_{2}(\bar{\Omega})}\right]
\end{aligned}
$$

hold, where $M$ does not depend on $f(t, x)$.

The proof of Theorem 119 is based on Theorem 117, the symmetry properties of the operator $A^{x}$ defined by formula (299), and Theorem 70 on the coercivity inequality for the solution of the elliptic differential problem in $L_{2}(\bar{\Omega})$. 
Let us associate initial-boundary value problem (294) with corresponding first order of accuracy difference scheme

$$
\begin{gathered}
\tau^{-2}\left(u_{k+1}-2 u_{k}+u_{k-1}\right)+A u_{k+1} \\
+\frac{1}{\sqrt{\pi}} \sum_{m=1}^{k} \frac{1}{(k-m) !} \int_{0}^{\infty} t^{k-m-1 / 2} e^{-t} d t \\
\cdot \frac{f_{m}-f_{m-1}}{\tau^{1 / 2}}=f_{k}, \\
f_{k}=f\left(t_{k}\right), \\
t_{k}=k \tau, \quad 1 \leq k \leq N-1, \quad N \tau=T ; \\
u_{0}=0, \\
\left(I+\tau^{2} A\right) \tau^{-1}\left(u_{1}-u_{0}\right)=\psi .
\end{gathered}
$$

Theorem 120 (see [96]). Suppose that $\psi \in D\left(A^{1 / 2}\right)$. Then, for the solution of difference scheme (301), the stability inequalities

$$
\begin{gathered}
\max _{0 \leq k \leq N}\left\|u_{k}\right\|_{H} \leq M\left\{\sum_{s=1}^{N-1}\left\|A^{-1 / 2} f_{s}\right\|_{H} \tau+\left\|A^{-1 / 2} \psi\right\|_{H}\right\}, \\
\max _{0 \leq k \leq N}\left\|A^{1 / 2} u_{k}\right\|_{H} \leq M\left\{\sum_{s=1}^{N-1}\left\|f_{s}\right\|_{H} \tau+\|\psi\|_{H}\right\}, \\
\max _{1 \leq k \leq N-1}\left\|\tau^{-2}\left(u_{k+1}-2 u_{k}+u_{k-1}\right)\right\|_{H}+\max _{0 \leq k \leq N}\left\|A u_{k}\right\|_{H} \\
\leq M_{1}\left\{\sum_{s=2}^{N-1}\left\|f_{s}-f_{s-1}\right\|_{H}+\left\|f_{1}\right\|_{H}+\left\|A^{1 / 2} \psi\right\|_{H}\right\}
\end{gathered}
$$

hold, where $M_{1}$ does not depend on $\tau, \psi$, or $f_{s}, 1 \leq s \leq N-1$.

First, initial-boundary value problem (297) for onedimensional fractional hyperbolic equation was considered. The discretization of problem (297) is carried out in two steps. In the first step, let us define the grid space

$$
[0,1]_{h}=\left\{x: x_{r}=r h, 0 \leq r \leq M, M h=1\right\} .
$$

We introduce the Hilbert space $L_{2 h}=L_{2}\left([0,1]_{h}\right)$ of the grid functions $\varphi^{h}(x)=\left\{\varphi^{r}\right\}_{1}^{M-1}$ defined on $[0,1]_{h}$, equipped with the norm

$$
\left\|\varphi^{h}\right\|_{L_{2 h}}=\left(\sum_{r=1}^{M-1}\left|\varphi^{h}(x)\right|^{2} h\right)^{1 / 2} .
$$

To the differential operator $A$ generated by problem (297), we assign the difference operator $A_{h}^{x}$ by the formula

$$
A_{h}^{x} \varphi^{h}(x)=\left\{-\left(a(x) \varphi_{\bar{x}}\right)_{x, n}+\delta \varphi_{n}\right\}_{1}^{M-1}
$$

acting in the space of grid functions $\varphi^{h}(x)=\left\{\varphi_{n}\right\}_{0}^{M}$ satisfying the conditions $\varphi_{0}=\varphi_{M}, \varphi_{1}-\varphi_{0}=\varphi_{M}-\varphi_{M-1}$. It is well-known that $A_{h}^{x}$ is a self-adjoint positive definite operator in $L_{2 h}$. With the help of $A_{h}^{x}$, we arrive the following initial value problem

$$
\begin{gathered}
\frac{d^{2} v^{h}(t, x)}{d t^{2}}+D_{t}^{1 / 2} v^{h}(t, x)+A_{h}^{x} v^{h}(t, x)=f^{h}(t, x), \\
0 \leq t \leq T, \quad x \in[0,1]_{h}, \\
v^{h}(0, x)=0, \quad v_{t}^{h}(0, x)=0, \quad x \in[0,1]_{h},
\end{gathered}
$$

for an infinite system of ordinary fractional differential equations.

In the second step, we replace problem (306) with difference scheme (301):

$$
\begin{gathered}
\frac{u_{k+1}^{h}(x)-2 u_{k}^{h}(x)+u_{k-1}^{h}(x)}{\tau^{2}} \\
+\frac{1}{\sqrt{\Pi}} \sum_{m=1}^{k} \frac{\Gamma(k-m+1 / 2)}{(k-m) !}\left(\frac{u_{m}^{h}-u_{m-1}^{h}}{\tau^{1 / 2}}\right) \\
+A_{h}^{x} u_{k+1}^{h}=f_{k}^{h}(x), \quad x \in[0,1]_{h}, \\
f_{k+1}^{h}(x)=f\left(t_{k+1}, x_{n}\right), \\
t_{k+1}=(k+1) \tau, \quad 1 \leq k \leq N-1, \quad N \tau=T, \\
\left(I+\tau^{2} A_{h}^{x}\right) \frac{u_{1}^{h}(x)-u_{0}^{h}(x)}{\tau}=0, \\
u_{0}^{h}(x)=0, \quad x \in[0,1]_{h} .
\end{gathered}
$$

Theorem 121 (see [96]). Let $\tau$ and $h$ be sufficiently small numbers. Then, the solutions of difference scheme (307) satisfy the following stability estimates:

$$
\begin{aligned}
\max _{0 \leq k \leq N}\left\|u_{k}^{h}\right\|_{L_{2 h}}+\max _{0 \leq k \leq N} \sum_{r=1}^{m}\left\|\left(u_{k}^{h}\right)_{x_{r}, j_{r}}\right\|_{L_{2 h}} \\
\leq M_{1} \max _{1 \leq k \leq N-1}\left\|f_{k}^{h}\right\|_{L_{2 h}}, \\
\max _{1 \leq k \leq N-1}\left\|\tau^{-2}\left(u_{k+1}^{h}-2 u_{k}^{h}+u_{k-1}^{h}\right)\right\|_{L_{2 h}} \\
\quad+\max _{0 \leq k \leq N} \sum_{r=1}^{m}\left\|\left(u_{k}^{h}\right)_{\bar{x}_{r} x_{r}, j_{r}}\right\|_{L_{2 h}} \\
\leq M_{1}\left[\left\|f_{1}^{h}\right\|_{L_{2 h}}+\max _{2 \leq k \leq N-1}\left\|\tau^{-1}\left(f_{k}^{h}-f_{k-1}^{h}\right)\right\|_{L_{2 h}}\right] .
\end{aligned}
$$

Here $M_{1}$ does not depend on $\tau, h$, or $f_{k}^{h}, 1 \leq k<N$.

The proof of Theorem 121 is based on abstract Theorem 117 and the symmetry properties of the operator $A_{h}^{x}$ defined by (305).

Second, initial-boundary value problem (299) for the $m$-dimensional hyperbolic equation is considered. The discretization of problem (299) is carried out in two steps. 
In the first step, let us define the grid sets

$$
\begin{gathered}
\bar{\Omega}_{h}=\left\{x=x_{r}=\left(h_{1} r_{1}, \ldots, h_{m} r_{m}\right), r=\left(r_{1}, \ldots, r_{m}\right),\right. \\
\left.0 \leq r_{j} \leq N_{j}, h_{j} N_{j}=1, j=1, \ldots, m\right\}, \\
\Omega_{h}=\bar{\Omega}_{h} \cap \Omega, \quad S_{h}=\bar{\Omega}_{h} \cap S .
\end{gathered}
$$

We introduce the Banach space $L_{2 h}=L_{2}\left(\bar{\Omega}_{h}\right)$ of the grid functions $\varphi^{h}(x)=\left\{\varphi\left(h_{1} r_{1}, \ldots, h_{m} r_{m}\right)\right\}$ defined on $\bar{\Omega}_{h}$, equipped with the norm

$$
\left\|\varphi^{h}\right\|_{L_{2}\left(\bar{\Omega}_{h}\right)}=\left(\sum_{x \in \bar{\Omega}_{h}}\left|\varphi^{h}(x)\right|^{2} h_{1} \cdots h_{m}\right)^{1 / 2} .
$$

To the differential operator $A$ generated by problem (299), we assign the difference operator $A_{h}^{x}$ by the formula

$$
A_{h}^{x} u_{x}^{h}=-\sum_{r=1}^{m}\left(a_{r}(x) u_{\bar{x}_{r}}^{h}\right)_{x_{r}, j_{r}}
$$

acting in the space of grid functions $u^{h}(x)$, satisfying the conditions $u^{h}(x)=0$ for all $x \in S_{h}$. It is known that $A_{h}^{x}$ is a self-adjoint positive definite operator in $L_{2}\left(\bar{\Omega}_{h}\right)$. With the help of $A_{h}^{x}$, we arrive at the initial-boundary value problem

$$
\begin{gathered}
\frac{d^{2} v^{h}(t, x)}{d t^{2}}+D_{t}^{1 / 2} v^{h}(t, x)+A_{h}^{x} v^{h}(t, x)=f^{h}(t, x), \\
0 \leq t \leq T, \quad x \in \Omega_{h}, \\
v^{h}(0, x)=0, \quad \frac{d v^{h}(0, x)}{d t}=0, \quad x \in \bar{\Omega},
\end{gathered}
$$

for an infinite system of ordinary fractional differential equations.

In the second step, we replace problem (312) with difference scheme (301):

$$
\begin{gathered}
\frac{u_{k+1}^{h}(x)-2 u_{k}^{h}(x)+u_{k-1}^{h}(x)}{\tau^{2}} \\
+\frac{1}{\sqrt{\Pi}} \sum_{m=1}^{k} \frac{\Gamma(k-m+1 / 2)}{(k-m) !}\left(\frac{u_{m}^{h}-u_{m-1}^{h}}{\tau^{1 / 2}}\right) \\
+A_{h}^{x} u_{k+1}^{h}=f_{k}^{h}(x), \quad x \in \bar{\Omega}_{h}, \\
f_{k+1}^{h}(x)=f\left(t_{k+1}, x_{n}\right), \\
t_{k+1}=(k+1) \tau, \quad 1 \leq k \leq N-1, \quad N \tau=T, \\
\left(I+\tau^{2} A_{h}^{x}\right) \frac{u_{1}^{h}(x)-u_{0}^{h}(x)}{\tau}=0, \\
u_{0}^{h}(x)=0, \quad x \in \bar{\Omega}_{h} .
\end{gathered}
$$

Theorem 122 (see $[94,96])$. Let $\tau$ and $|h|$ be sufficiently small numbers. Then, the solutions of difference scheme (313) satisfy the following stability estimates:

$$
\begin{aligned}
& \max _{0 \leq k \leq N}\left\|u_{k}^{h}\right\|_{L_{2 h}}+\max _{0 \leq k \leq N} \sum_{r=1}^{m}\left\|\left(u_{k}^{h}\right)_{x_{r}, j_{r}}\right\|_{L_{2 h}} \\
& \leq M_{1} \max _{1 \leq k \leq N-1}\left\|f_{k}^{h}\right\|_{L_{2 h}}, \\
& \max _{1 \leq k \leq N-1}\left\|\tau^{-2}\left(u_{k+1}^{h}-2 u_{k}^{h}+u_{k-1}^{h}\right)\right\|_{L_{2 h}} \\
& \quad+\max _{0 \leq k \leq N} \sum_{r=1}^{m}\left\|\left(u_{k}^{h}\right)_{\bar{x}_{r} x_{r}, j_{r}}\right\|_{L_{2 h}} \\
& \leq M_{1}\left[\left\|f_{1}^{h}\right\|_{L_{2 h}}+\max _{2 \leq k \leq N-1}\left\|\tau^{-1}\left(f_{k}^{h}-f_{k-1}^{h}\right)\right\|_{L_{2 h}}\right] .
\end{aligned}
$$

Here $M_{1}$ does not depend on $\tau$, $h$, or $f_{k}^{h}, 1 \leq k<N$.

The proof of Theorem 122 is based on abstract Theorem 117, the symmetry properties of the operator $A_{h}^{x}$ defined by formula (311), and Theorem 76 on the coercivity inequality for the solution of the elliptic difference problem in $L_{2 h}$.

In [94], a procedure of modified Gauss elimination method was used for obtaining the solution of difference scheme (313) in the case of one-dimensional fractional hyperbolic partial differential equations. The theoretical statements for the solution of this difference scheme were supported by the results of the numerical experiment.

In $[95,97]$, the numerical and analytic solutions of the mixed problem for multidimensional fractional hyperbolic partial differential equations with the Neumann condition were presented. The stable difference scheme for the numerical solution of the mixed problem for the multidimensional fractional hyperbolic equation with the Neumann condition was presented. Stability estimates for the solution of this difference scheme and for the first and second order difference derivatives were obtained. A procedure of modified Gauss elimination method was used for solving this difference scheme in the case of one-dimensional fractional hyperbolic partial differential equations. He's variational iteration method was applied. The comparison of these methods was presented. Application of variational iteration technique to this problem has shown the rapid convergence of the sequence constructed by this method to the exact solution.

Finally, in [127], the initial-boundary value problem for partial differential equations of higher order involving the Caputo fractional derivative was studied. Theorems on existence and uniqueness of a solution and its continuous dependence on the initial data and on the right-hand side of the equation were established.

7.7. Singular Perturbation Hyperbolic Problems. We consider the abstract Cauchy problem for hyperbolic equations:

$$
\begin{gathered}
\varepsilon^{2} v^{\prime \prime}(t)+A v(t)=f(t) \quad(0 \leq t \leq T), \\
v(0)=\varphi, \quad v^{\prime}(0)=\psi
\end{gathered}
$$


in Hilbert space $H$ with the self -adjoint positive definite operator $A$ and $\varepsilon \in(0, \infty)$.

Function $v(t)$ is called a solution of problem (315) if the following conditions are satisfied:

(i) $v(t)$ is twice continuously differentiable on the segment $[0, T]$.

(ii) The element $v(t)$ belongs to $D(A)$ for all $t \in[0, T]$ and the function $A v(t)$ is continuous on the segment $[0, T]$.

(iii) $v(t)$ satisfies the equations and initial conditions (109).

If the function $f(t)$ is not only continuous, but also continuously differentiable on $[0, T], \varphi \in D(A)$, and $\psi \in$ $D\left(A^{1 / 2}\right)$, it is easy to show that the formula

$$
\begin{aligned}
v(t)= & C\left(\frac{t}{\varepsilon}, A\right) v_{0}+S\left(\frac{t}{\varepsilon}, A\right) v_{0}^{\prime} \\
& +\frac{1}{\varepsilon^{2}} \int_{0}^{t} S\left(\frac{t-s}{\varepsilon}, A\right) f(s) d s
\end{aligned}
$$

gives a solution of problem (315). Here

$$
\begin{gathered}
C\left(\frac{t}{\varepsilon}, A\right)=\frac{e^{i(t / \varepsilon) A^{1 / 2}}+e^{-i(t / \varepsilon) A^{1 / 2}}}{2}, \\
S\left(\frac{t}{\varepsilon}, A\right)=\varepsilon A^{-1 / 2} \frac{e^{i(t / \varepsilon) A^{1 / 2}}-e^{-i(t / \varepsilon) A^{1 / 2}}}{2 i} .
\end{gathered}
$$

Theorem 123 (see [99]). Assume that the function $f(t)$ has $2 m+3$ derivatives and

$$
\left\|f^{(2 m+3)}(t)\right\|_{H} \leq M, \quad 0 \leq t \leq T .
$$

Then for small $\varepsilon$ and an even number $m$ the following $(m+2)$ th order asymptotic formula for the solution of (316) holds:

$$
v(t)=\sum_{i=0}^{m} \varepsilon^{i}\left[u_{i}(t)+w_{i}\left(\frac{t}{\varepsilon}\right)\right]+o\left(\varepsilon^{m+2}\right) ;
$$

for small $\varepsilon$ and an odd number $m$ the following $(m+1)$ th order asymptotic formula for the solution of (316) holds:

$$
v(t)=\sum_{i=0}^{m} \varepsilon^{i}\left[u_{i}(t)+w_{i}\left(\frac{t}{\varepsilon}\right)\right]+o\left(\varepsilon^{m+1}\right),
$$

where $u_{i}(t)$ and $w_{i}(t / \varepsilon)$ for $i \in\{1, \ldots, m\}$ are defined by the following formulas

$$
\begin{gathered}
u_{0}(t)=A^{-1} f(t), \quad u_{1}(t)=0, \\
u_{i}(t)=-A^{-1} u_{i-2}^{\prime \prime}(t), \quad i=2, \ldots, m, \\
\left(w_{i}^{\prime \prime}\right)_{\xi \xi}(\xi)+A w_{i}(\xi)=0, \quad i=0,1, \ldots, m, \\
w_{0}(0)=\varphi-u_{0}(0), \\
w_{i}(0)=-u_{i}(0), \quad i=1, \ldots, m, \\
\left(w_{0}^{\prime}\right)_{\xi}(0)=0, \quad\left(w_{1}^{\prime}\right)_{\xi}(0)=\psi-u_{0}^{\prime}(0), \\
\left(w_{i}^{\prime}\right)_{\xi}(0)=-u_{i-1}^{\prime}(0), \quad i=2, \ldots, m .
\end{gathered}
$$

In Section 7.1, the stability of the high order of accuracy difference schemes generated by an exact difference scheme or by Taylor's decomposition on the three points for the numerical solutions of abstract initial value problem (315) for $\varepsilon=1$ was presented. Unfortunately, these difference schemes can not be applied for the approximate solutions of (315) in the general cases $\varepsilon \in(0, \infty)$. In $[100,101]$, the high order of accuracy two-step uniform difference schemes of the approximate solutions for differential equations of the hyperbolic type with arbitrary parameter $\varepsilon$ at the highest derivative was presented. The stability estimates of the solutions of these difference schemes were obtained.

By Theorem 71, we have the following exact two-step difference scheme:

$$
\begin{gathered}
v\left(t_{k+1}\right)-2 C\left(\frac{\tau}{\varepsilon}, A\right) v\left(t_{k}\right)+v\left(t_{k-1}\right) \\
=\frac{1}{\varepsilon^{2}}\left\{\int_{t_{k}}^{t_{k+1}} S\left(\frac{t_{k+1}-z}{\varepsilon}, A\right) f(z) d z\right. \\
\left.+\int_{t_{k-1}}^{t_{k}} S\left(\frac{z-t_{k-1}}{\varepsilon}, A\right) f(z) d z\right\}, \\
\quad 1 \leq k \leq N-1, \\
v(0)=v_{0},
\end{gathered}
$$

$$
\begin{aligned}
v(\tau) & -v(0) \\
= & \left(C\left(\frac{\tau}{\varepsilon}, A\right)-I\right) v(0) \\
& +S\left(\frac{\tau}{\varepsilon}, A\right) v^{\prime}(0)+\frac{1}{\varepsilon^{2}} \int_{0}^{\tau} S\left(\frac{\tau-z}{\varepsilon}, A\right) f(z) d z
\end{aligned}
$$

or

$$
\begin{gathered}
\rho^{-2}\left(v\left(t_{k+1}\right)-2 v\left(t_{k}\right)+v\left(t_{k-1}\right)\right) \\
=2 \rho^{-2}\left(C\left(\frac{\tau}{\varepsilon}, A\right)-I\right) v\left(t_{k}\right)+\tau^{-1} \varepsilon^{2} \tilde{f}_{k}, \\
\tilde{f}_{k}=\tilde{f}_{1, k+1}+S\left(\frac{\tau}{\varepsilon}, A\right) \tilde{f}_{2, k}-C\left(\frac{\tau}{\varepsilon}, A\right) \tilde{f}_{1, k}, \\
\tilde{f}_{1, k}=\left(\varepsilon^{2} \tau\right)^{-1} \int_{t_{k-1}}^{t_{k}} S\left(\frac{t_{k}-z}{\varepsilon}, A\right) f(z) d z, \\
\tilde{f}_{2, k}=\left(\varepsilon^{2} \tau\right)^{-1} \int_{t_{k-1}}^{t_{k}} C\left(\frac{t_{k}-z}{\varepsilon}, A\right) f(z) d z, \\
u_{0}=v_{0}, \\
u_{1}=C\left(\frac{\tau}{\varepsilon}, A\right) v(0)+S\left(\frac{\tau}{\varepsilon}, A\right) v^{\prime}(0)+\tau \tilde{f}_{1,1} .
\end{gathered}
$$


In $[100,101]$, applying this exact two-step difference scheme, the following high order of accuracy difference schemes

$$
\begin{gathered}
\rho^{-2}\left(u_{k+1}-2 u_{k}+u_{k-1}\right) \\
=2 \rho^{-2}(C(\rho, A)-I) u_{k}+\tau^{-1} \varepsilon^{2} f_{k}, \\
f_{k}=f_{1, k+1}+S(\rho, A) f_{2, k}-C(\rho, A) f_{1, k}, \\
u_{0}=v_{0}, \\
u_{1}=C(\rho, A) v(0)+S(\rho, A) v^{\prime}(0)+\tau f_{1,1}, \\
f_{1, k}=(\tau A)^{-1}\left[f\left(t_{k}\right)-C(\rho, A) f\left(t_{k-1}\right)\right] \\
-(\tau A)^{-1} \sum_{j=0}^{m-1} B_{j} f^{(j+1)}\left(t_{k-1}\right), \\
f_{j, k}=-\left(\varepsilon^{2} \tau\right)^{-1} S(\rho, A) f\left(t_{k-1}\right) \\
+\left(\varepsilon^{2} \tau\right)^{-1} \sum_{j=0}^{m-1} C_{j} f^{(j+1)}\left(t_{k-1}\right), \\
B_{2 j+1}=\left(\varepsilon^{2} A^{-1}\right)^{j} B_{1}-\sum_{i=1}^{j}\left(\varepsilon^{2} A^{-1}\right){ }^{j-i+1} \frac{\tau^{2 i}}{(2 i) !}, \\
\left.B_{0}=S(\rho, A), \quad \varepsilon_{1}^{2} A\right)^{-1} \frac{\tau^{j}}{j !}, \quad 1 \leq j \leq m-1 \\
B_{2 j}=\left(\varepsilon^{2} A^{-1}\right)^{j} B_{0}-\sum_{i=1}^{j}\left(\varepsilon^{2} A^{-1}\right)^{j-i+1} \frac{\tau^{2 i-1}}{(2 i-1) !},
\end{gathered}
$$

was constructed for the approximate solutions of initial value problem (315). Here $\tau=\rho \varepsilon$.

Theorem 124 (see [101]). For the solution of two-step difference scheme (324), the following stability inequality holds:

$$
\begin{gathered}
\max _{0 \leq k \leq N}\left\|u_{k}\right\|_{H} \leq M\left[\max _{1 \leq k \leq N}\left\|f_{1, k}\right\|_{H}+\varepsilon \max _{1 \leq k \leq N}\left\|A^{-1 / 2} f_{2, k}\right\|_{H}\right. \\
\left.+\varepsilon\left\|A^{-1 / 2} v_{0}^{\prime}\right\|_{H}+\left\|v_{0}\right\|_{H}\right]
\end{gathered}
$$

where $M$ does not depend on $\varepsilon, \tau, f_{1, k}, f_{2, k}, 1 \leq k \leq N$, or $v_{0}$, $v_{0}^{\prime}$.

Stability inequality (325) permits us to obtain the estimate of convergence of two-step difference scheme (324).
Theorem 125 (see [101]). Suppose that the function $f(t)$ has $m+1$ derivatives and

$$
\left\|f^{(m+1)}(t)\right\|_{H} \leq M, \quad 0 \leq t \leq T .
$$

Then, for the solution of difference problem (324), the following convergence estimate is valid:

$$
\max _{1 \leq k \leq N}\left\|v\left(t_{k}\right)-u_{k}\right\|_{H} \leq M_{1} \tau^{m}
$$

where $M_{1}$ does not depend on $\tau$ or $\varepsilon$.

In $[98,100,101]$, applying this approach, the high order of accuracy uniform difference schemes for the following three types of singular perturbation problems

$$
\begin{gathered}
\varepsilon^{2} v^{\prime \prime}(t)+v^{\prime}(t)=A v(t)+f(t) \quad(0 \leq t \leq T), \\
v(0)=\varphi, \quad v^{\prime}(0)=\psi, \\
\varepsilon^{2} v^{\prime \prime}(t)-i v^{\prime}(t)=A v(t)+f(t) \quad(0 \leq t \leq T), \\
v(0)=\varphi, \quad v^{\prime}(0)=\psi, \\
\varepsilon^{2} v^{\prime \prime}(t)+v^{\prime}(t)=\left(\varepsilon^{2} A+B\right) v(t)+f(t) \quad(0 \leq t \leq T), \\
v(0)=\varphi, \quad v^{\prime}(0)=\psi
\end{gathered}
$$

involving second order differential equations in Banach space $E$ was presented and investigated. Here $A, B$ are linear, generally unbounded operators in $E$. Theorems on the stability estimates of the solutions of these difference schemes were established.

\section{Conflict of Interests}

The authors declare that there is no conflict of interests regarding the publication of this paper.

\section{Acknowledgment}

Research was partially supported by Russian Foundation for Basic Research (13-01-00096-a and 15-01-00026-a).

\section{References}

[1] A. Ashyralyev and P. E. Sobolevskii, Well-Posedness of Parabolic Difference Equations, vol. 69 of Operator Theory Advances and Applications, Birkhäuser, Basel, Switzerland, 1994.

[2] A. Ashyralyev and P. E. Sobolevskii, New Difference Schemes for Partial Differential Equations, Operator Theory: Advances and Applications, Birkhäuser, Basel, Switzerland, 2004.

[3] D. Guidetti, "Backward euler scheme, singular hölder norms, and maximal regularity for parabolic difference equations," Numerical Functional Analysis and Optimization, vol. 28, no. 34, pp. 307-337, 2007.

[4] V. Keyantuo and C. Lizama, "Maximal regularity for a class of integro-differential equations with infinite delay in Banach spaces," Studia Mathematica, vol. 168, no. 1, pp. 25-50, 2005. 
[5] V. Keyantuo and C. Lizama, "Hölder continuous solutions for integro-differential equations and maximal regularity," Journal of Differential Equations, vol. 230, no. 2, pp. 634-660, 2006.

[6] S. Piskarev, Differential Equations in Banach Space and It's Approximation, Moscow State University Publish House, Moscow, Russia, 2005, (Russian).

[7] A. Ashyralyev and P. E. Sobolevskii, "Two new approaches for construction of the high order of accuracy difference schemes for hyperbolic differential equations," Discrete Dynamics in Nature and Society, vol. 2005, no. 2, pp. 183-213, 2005.

[8] P. Clement and S. Guerre-Delabrire, "On the regularity of abstract Cauchy problems and boundary value problems," Atti della Accademia Nazionale dei Lincei. Classe di Scienze Fisiche, Matematiche e Naturali. Rendiconti Lincei. Matematica e Applicazioni, vol. 9, no. 4, pp. 245-266, 1998.

[9] R. H. W. Hoppe, "Discrete approximations of cosine operator functions I," SIAM Journal on Numerical Analysis, vol. 19, no. 6, pp. 1110-1128, 1982.

[10] V. Keyantuo and C. Lizama, "Periodic solutions of second order differential equations in Banach spaces," Mathematische Zeitschrift, vol. 253, no. 3, pp. 489-514, 2006.

[11] A. A. Samarskii, I. P. Gavrilyuk, and V. L. Makarov, "Stability and regularization of three-level difference schemes with unbounded operator coefficients in Banach spaces," SIAM Journal on Numerical Analysis, vol. 39, no. 2, pp. 708-723, 2002.

[12] A. Ashyralyev, C. Cuevas, and S. Piskarev, "On well-posedness of difference schemes for abstract elliptic problems in $L^{p}([0 ; T] ; E)$ spaces," Numerical Functional Analysis and Optimization, vol. 29, no. 1-2, pp. 43-65, 2008.

[13] V. V. Vasil'ev and S. I. Piskarev, "Differential equations in Banach spaces. II. Theory of cosine operator functions," Journal of Mathematical Sciences, vol. 122, no. 2, pp. 3055-3174, 2004.

[14] D. Guidetti, B. Karasözen, and S. Piskarev, "Approximation of abstract differential equations," Journal of Mathematical Sciences, vol. 122, no. 2, pp. 3013-3054, 2004.

[15] B. Eberhardt and G. Greiner, "Baillon's theorem on maximal regularity," Acta Applicandae Mathematicae, vol. 27, pp. 4754, 1992, Positive operators and semigroups on Banach lattices (Curaçao, 1990).

[16] G. da Prato and P. Grisvard, "Sommes d’opérateus linéaires et équations différentielles opérationnelles," Journal de Mathématiques Pures et Appliquées, vol. 54, no. 3, pp. 305-387, 1975.

[17] G. da Prato and P. Grisvard, "Équations d'évolution abstraites non linéaires de type parabolique," Comptes Rendus de l'Académie des Sciences-Série A-B, vol. 283, no. 9, pp. A709A711, 1976.

[18] P. E. Sobolevskii, "Some properties of the solutions of differential equations in fractional spaces," Trudy NaucnoIssledovatel'skogi Instituta Matematiki VGU, vol. 74, pp. 68-76, 1975 (Russian).

[19] N. J. Kalton and G. Lancien, "A solution to the problem of $L^{P}-$ maximal regularity," Mathematische Zeitschrift, vol. 235, no. 3, pp. 559-568, 2000.

[20] P. C. Kunstmann and L. Weis, "Maximal $L_{p}$-regularity for parabolic equations, Fourier multiplier theorems and $H^{\infty}$ functional calculus," in Functional Analytic Methods for Evolution Equations, vol. 1855 of Lecture Notes in Mathematics, pp. 65-311, Springer, Berlin, Germany, 2004.

[21] L. Weis, "Operator-valued Fourier multiplier theorems and maximal $L_{p}$-regularity," Mathematische Annalen, vol. 319, no. 4 , pp. 735-758, 2001.
[22] H. O. Fattorini, Second Order Linear Differential Equations in Banach Spaces, Elsevier Science Publishing, North-Holland, 1985.

[23] J. Kisynski, "On cosine operator functions and one parameter groups of operators," Studia Mathematica, vol. 44, pp. 93-105, 1972.

[24] D. Orlovsky, S. Piskarev, and R. Spigler, "On approximation of inverse problems for abstract hyperbolic equations," Taiwanese Journal of Mathematics, vol. 14, no. 3, pp. 1145-1167, 2010.

[25] V. V. Vasil'ev, S. G. Krein, and S. Piskarev, "Operator semigroups, cosine operator functions, and linear differential equations," Itogi Nauki i Tekhniki: Seriya "Matematicheskii Analiz", vol. 28, pp. 87-202, 1990 (Russian), Akademiya Nauk SSSR Vserossiiskii Institut Nauchoi i Tekhnicheskoi Informatsii, Moscow, translated in Journal of Soviet Mathematics, vol. 54, no. 4, pp. $1042-$ 1129, 1991.

[26] C. C. Travis and G. F. Webb, "Cosine families and abstract nonlinear second order differential equations," Acta Mathematica Academiae Scientiarum Hungaricae, vol. 32, no. 1-2, pp. 75-96, 1978.

[27] I. Cioranescu and V. Keyantuo, "On operator cosine functions in UMD paces," Semigroup Forum, vol. 63, no. 3, pp. 429-440, 2001.

[28] C. C. Travis and G. F. Webb, "Second order differential equations in Banach space," in Proceedings of the International Symposium on Nonlinear Equations in Abstract Spaces, pp. 331361, 1978.

[29] S. G. Krein, Linear Differential Equations in Banach Space, vol. 29 of Translations of Mathematical Monographs, American Mathematical Society, Providence, RI, USA, 1971, Translated from the Russian by J. M. Danskin.

[30] D.-K. Chyan, S.-Y. Shaw, and S. Piskarev, "On maximal regularity and semivariation of cosine operator functions," Journal of the London Mathematical Society, vol. 59, no. 3, pp. 1023-1032, 1999.

[31] C. Palencia and S. Piskarev, "On multiplicative perturbations of $\mathrm{C}_{0}$-groups and $\mathrm{C}_{0}$-cosine operator functions," Semigroup Forum, vol. 63, no. 2, pp. 127-152, 2001.

[32] D. Dier and E. M. Ouhabaz, "Maximal regularity for nonautonomous second order Cauchy problems," Integral Equations and Operator Theory, vol. 78, no. 3, pp. 427-450, 2014.

[33] W. Arendt, R. Chill, S. Fornaro, and C. Poupaud, " $L_{p}$-maximal regularity for non-autonomous evolution equations," Journal of Differential Equations, vol. 237, no. 1, pp. 1-26, 2007.

[34] V. Poblete, "Maximal regularity of second-order equations with delay," Journal of Differential Equations, vol. 246, no. 1, pp. 261276, 2009.

[35] S. Q. Bu, "Maximal regularity of second order delay equations in Banach spaces," Acta Mathematica Sinica, vol. 25, no. 1, pp. 21-28, 2009.

[36] S. Q. Bu and Y. Fang, "Maximal regularity of second order delay equations in Banach spaces," Science China Mathematics, vol. 53, no. 1, pp. 51-62, 2010.

[37] Y. Z. Huang and M. C. Wang, "Analyticity of the sine family with $L^{P}$-maximal regularity for second order Cauchy problems," Acta Mathematica Sinica, English Series, vol. 26, no. 3, pp. 561-568, 2010.

[38] A. Ashyralyev, M. Martinez, J. Pastor, and S. Piskarev, "On wellposedness of abstact hyperbolic problem in function spaces," in Proceedings of the WSPC, pp. 679-688, 2009. 
[39] M. Sova, "Cosine operator functions," Rozprawy Matematyczne, vol. 49, pp. 1-47, 1966.

[40] P. E. Sobolevskii, "The theory of semigroups and the stability of difference schemes," in Operator Theory in Function Spaces (Proc. School, Novosibirsk, 1975) (Russian), pp. 304-307, "Nauka" Sibirskoe Otdelenie, Novosibirsk, Russia, 1977.

[41] P. Grisvard, Elliptic Problems in Nonsmooth Domains, Classics in Applied Mathematics, Pitman, Boston, Mass, USA, 1985.

[42] F. Z. Mezeghrani, "Necessary and sufficient conditions for the solvability and maximal regularity of abstract differential equations of mixed type in UMD spaces," Tsukuba Journal of Mathematics, vol. 35, no. 2, pp. 185-202, 2011.

[43] F. Z. Mezeghrani, "Necessary and sufficient conditions for the solvability and maximal regularity of abstract differential equations of mixed type in Hölder spaces," Osaka Journal of Mathematics, vol. 50, no. 3, pp. 725-747, 2013.

[44] R. D. Grigorieff, "Diskrete approximation von eigenwertproblemen. II. konvergenzordnung," Numerische Mathematik, vol. 24, no. 5, pp. 415-433, 1975.

[45] F. Stummel, "Diskrete Konvergenz linearer Operatoren III," in Linear Operators and Approximation: Proceedings of the Conference held at the Oberwolfach Mathematical Research Institute, Black Forest, August 14-22, 1971, vol. 20 of International Series of Numerical Mathematics, pp. 196-216, Birkhäuser, Berlin, Germany, 1972.

[46] G. Vaĭnikko, Funktionalanalysis der Diskretisierungsmethoden, Teubner-Texte zur Mathematik, B. G. Teubner, Leipzig, Germany, 1976.

[47] G. Vainikko, "Approximative methods for nonlinear equations (two approaches to the convergence problem)," Nonlinear Analysis, vol. 2, no. 6, pp. 647-687, 1978.

[48] S. Piskarev, "Approximation of holomorphic semigroups," Tartu Riikliku Ülikooli Toimetised, vol. 492, pp. 3-14, 1979.

[49] A. Ashyralyev and P. E. Sobolevskii, "The linear operator interpolation theory and the stability of difference schemes," Doklady Akademii nauk SSSR, vol. 275, no. 6, pp. 1289-1291, 1984 (Russian).

[50] P. E. Sobolevskii, "The coercive solvability of difference equations," Doklady Akademii Nauk SSSR, vol. 201, pp. 1063-1066, 1971.

[51] C. Le Merdy, "Counterexamples on $L_{p}$-maximal regularity," Mathematische Zeitschrift, vol. 230, no. 1, pp. 47-62, 1999.

[52] A. Ashyralyev, S. Piskarev, and L. Weis, "On well-posedness of difference schemes for abstract parabolic equations in $L^{P}([0$, T];E) spaces," Numerical Functional Analysis and Optimization, vol. 23, no. 7-8, pp. 669-693, 2002.

[53] S. Piskarev, "Solution of a second order evolution equation under the Krein Fattorini conditions," Differential Equations, vol. 21, pp. 1100-1106, 1985.

[54] S. Piskarev, "Discretisation of abstract hyperbolic equation," Tartu Riikliku Ulikooli Toimetised, vol. 500, pp. 3-23, 1979.

[55] W. Chojnacki, "Group representations of bounded cosine functions," Journal fur die Reine und Angewandte Mathematik, no. 478, pp. 61-84, 1996.

[56] W. Chojnacki, "On group decompositions of bounded cosine sequences," Studia Mathematica, vol. 181, no. 1, pp. 61-85, 2007.

[57] R. K. Mohanty, "An operator splitting method for an unconditionally stable difference scheme for a linear hyperbolic equation with variable coefficients in two space dimensions," Applied Mathematics and Computation, vol. 152, no. 3, pp. 799806, 2004.
[58] R. K. Mohanty, "An unconditionally stable finite difference formula for a linear second order one space dimensional hyperbolic equation with variable coefficients," Applied Mathematics and Computation, vol. 165, no. 1, pp. 229-236, 2005.

[59] R. K. Mohanty, "An operator splitting technique for an unconditionally stable difference method for a linear three space dimensional hyperbolic equation with variable coefficients," Applied Mathematics and Computation, vol. 162, no. 2, pp. 549557, 2005.

[60] S. Piskarev, "Stability of difference schemes in Cauchy problems with almost periodic solutions," Differentsial'nye Uravneniya, vol. 20, no. 4, pp. 689-695, 1984 (Russian).

[61] S. Piskarev, "Principles of discretization methods III," Report ak-3410, Acoustic Institute, Academy of Science USSR, 1986 (Russian).

[62] P. E. Sobolevskii and L. M. Chebotaryeva, "Approximate solution by method of lines of the Cauchy problem for an abstract hyperbolic equations," Izvestiya Vysših Učebnyh Zavedeniu Matematika, no. 5, pp. 103-116, 1977 (Russian).

[63] A. Ashyralyev and P. E. Sobolevskii, "A note on the difference schemes for hyperbolic equations," Abstract and Applied Analysis, vol. 6, no. 2, pp. 63-70, 2001.

[64] A. Ashyralyev and M. Koksal, "On the second order of accuracy difference scheme for hyperbolic equations in a hilbert space," Numerical Functional Analysis and Optimization, vol. 26, no. 78, pp. 739-772, 2005.

[65] A. Ashyralyev and M. E. Koksal, "Stability of a second order of accuracy difference scheme for hyperbolic equation in a Hilbert space," Discrete Dynamics in Nature and Society, vol. 2007, Article ID 57491, 25 pages, 2007.

[66] A. Ashyralyev and M. E. Köksal, "A numerical solution of wave equation arising in non-homogeneous cylindrical shells," Turkish Journal of Mathematics, vol. 32, no. 4, pp. 407-419, 2008.

[67] A. Ashyralyev and M. E. Koksal, "On the numerical solution of hyperbolic PDEs with variable space operator," Numerical Methods for Partial Differential Equations, vol. 25, no. 5, pp. 1086-1099, 2009.

[68] A. Ashyralyev, M. E. Koksal, and R. P. Agarwal, "A difference scheme for Cauchy problem for the hyperbolic equation with self-adjoint operator," Mathematical and Computer Modelling, vol. 52, no. 1-2, pp. 409-424, 2010.

[69] A. Ashyralyev, M. E. Koksal, and R. P. Agarwal, "An operatordifference scheme for abstract Cauchy problems," Computers and Mathematics with Applications, vol. 61, no. 7, pp. 1855-1872, 2011.

[70] A. Ashyralyev and N. Aggez, "Finite difference method for hyperbolic equations with the nonlocal integral condition," Discrete Dynamics in Nature and Society, vol. 2011, Article ID 562385, 15 pages, 2011.

[71] A. Ashyralyev and N. Aggez, "On the solution of NBVP for multidimensional hyperbolic equations," The Scientific World Journal, vol. 2014, Article ID 841602, 22 pages, 2014.

[72] A. Ashyralyev and O. Yildirim, "On multipoint nonlocal boundary value problems for hyperbolic differential and difference equations," Taiwanese Journal of Mathematics, vol. 14, no. 1, pp. 165-194, 2010.

[73] A. Ashyralyev and O. Yildirim, "High order of accuracy stable difference schemes for numerical solutions of NBVP for hyperbolic equations," AIP Conference Proceedings, vol. 1470, pp. 8487, 2012. 
[74] A. Ashyralyev and O. Yildirim, "A note on the second order of accuracy stable difference schemes for the nonlocal boundary value hyperbolic problem," Abstract and Applied Analysis, vol. 2012, Article ID 846582, 29 pages, 2012.

[75] A. Ashyralyev and O. Yildirim, "On stability of a third order of accuracy difference scheme for hyperbolic nonlocal BVP with self-adjoint operator," Abstract and Applied Analysis, vol. 2013, Article ID 959216, 15 pages, 2013.

[76] O. Yildirim and M. Uzun, "High order of accuracy stable difference schemes for the approximate solution of the multipoint NBVP for the hyperbolic equation," AIP Conference Proceedings, vol. 1611, no. 1, pp. 305-309, 2014.

[77] A. Ashyralyev, G. Judakova, and P. E. Sobolevskii, "A note on the difference schemes for hyperbolic-elliptic equations," Abstract and Applied Analysis, vol. 2006, Article ID 14816, 13 pages, 2006.

[78] A. Ashyralyev and I. Muradov, "On stability estimation of difference scheme of a first order of accuracy for hyperbolicparabolic equations," Izvestiya Akademii Nauk Turkmenistana. Seriya Fiziko-Tekhnicheskikh, Khimicheskikh i Geologicheskikh Nauk, no. 2, pp. 1-6, 1996 (Russian).

[79] A. Ashyralyev and I. Muradov, "On difference schemes second order of accuracy for hyperbolic-parabolic equations," in Modeling Processes of Exploitation of Gas Places and Applied Problems of Theoretical Gasohydrodynamics, pp. 127-138, Ilim, Ashgabat, Turkmenistan, 1998, (Russian).

[80] A. Ashyralyev and M. B. Orazov, "Theory of operators and the methods of solutions of boundary value problems for equations of mathematical physics," in 8 Years Turkmenistan's Independence, pp. 222-228, Ilim, Ashgabat, Turkmenistan, 1999, (Russian).

[81] A. Ashyralyev and M. B. Orazov, "The theory of operators and the stability of difference schemes for partial differential equations mixed types," Firat Üniversitesi Fen ve Mühendislik Bilimleri Dergisinde, vol. 11, no. 3, pp. 249-252, 1999.

[82] A. Ashyralyev and Y. Ozdemir, "Stability of difference schemes for hyperbolic-parabolic equations," Computers and Mathematics with Applications, vol. 50, no. 8-9, pp. 1443-1476, 2005.

[83] A. Ashyralyev and Y. Ozdemir, "A note on nonlocal boundary value problem for hyperbolic-parabolic equations," Functional Differential Equations, vol. 12, no. 1-2, pp. 67-81, 2005.

[84] A. Ashyralyev and Y. Ozdemir, "On nonlocal boundary value problems for hyperbolic-parabolic equations," Taiwanese Journal of Mathematics, vol. 11, no. 4, pp. 1075-1089, 2007.

[85] A. Ashyralyev and Y. Ozdemlr, "On stable implicit difference scheme for hyperbolic-parabolic equations in a Hilbert space," Numerical Methods for Partial Differential Equations, vol. 25, no. 5, pp. 1100-1118, 2009.

[86] A. Ashyralyev and F. Özger, "The hyperbolic-elliptic equation with the nonlocal condition," Mathematical Methods in the Applied Sciences, vol. 37, no. 4, pp. 524-545, 2014.

[87] A. Ashyralyev and A. Yurtsever, "On a nonlocal boundary value problem for semilinear hyperbolic-parabolic equations," Nonlinear Analysis: Theory, Methods \& Applications, vol. 47, no. 5, pp. 3585-3592, 2001.

[88] A. Ashyralyev and H. A. Yurtsever, "A note on the second order of accuracy difference schemes for hyperbolic-parabolic equations," Applied Mathematics and Computation, vol. 165, no. 3, pp. 517-537, 2005.

[89] A. Ashyralyev and H. A. Yurtsever, "The stability of difference schemes of second-order of accuracy for hyperbolic-parabolic equations," Computers and Mathematics with Applications, vol. 52, no. 3-4, pp. 259-268, 2006.
[90] Y. Ozdemir and M. Kucukunal, "Numerical solution of hyperbolic-Schrödinger equations with nonlocal boundary condition," in 1st International Conference on Analysis and Applied Mathematics (ICAAM '12), vol. 1470 of AIP Conference Proceedings, pp. 214-217, Gumushane, Turkey, October 2012.

[91] Y. Ozdemir and M. Kucukunal, "A note on nonlocal boundary value problems for hyperbolic schrödinger equations," Abstract and Applied Analysis, vol. 2012, Article ID 687321, 12 pages, 2012.

[92] N. Aggez and M. Ashyralyyewa, "Numerical solution of stochastic hyperbolic equations," Abstract and Applied Analysis, vol. 2012, Article ID 824819, 20 pages, 2012.

[93] A. Ashyralyev and M. Akat, "An approximation of stochastic hyperbolic equations: case with Wiener process," Mathematical Methods in the Applied Sciences, vol. 36, no. 9, pp. 1095-1106, 2013.

[94] A. Ashyralyev and F. Dal, "Finite difference and iteration methods for fractional hyperbolic partial differential equations with the neumann condition," Discrete Dynamics in Nature and Society, vol. 2012, Article ID 434976, 15 pages, 2012.

[95] A. Ashyralyev, F. Dal, and Z. Pinar, "On the numerical solution of fractional hyperbolic partial differential equations," Mathematical Problems in Engineering, vol. 2009, Article ID 730465, 11 pages, 2009.

[96] A. Ashyralyev, F. Dal, and Z. Pinar, "A note on the fractional hyperbolic differential and difference equations," Applied Mathematics and Computation, vol. 217, no. 9, pp. 4654-4664, 2011.

[97] F. Dal, "Application of variational iteration method to fractional hyperbolic partial differential equations," Mathematical Problems in Engineering, vol. 2009, Article ID 824385, 10 pages, 2009.

[98] A. Ashyralyev, Method of positive operators of investigations of the high order of accuracy difference schemes for parabolic and elliptic equations [Doctor of Sciences Thesis], Institute of Mathematics, Academic Sciences, Kiev, Ukraine, 1991, (Russian).

[99] A. Ashyralyev, Computational Mathematics, M. Auezov South Kazakhstan State University, Shymkent, Kazakhstan, 2013.

[100] A. Ashyralyev and H. Fattorini, "On difference schemes of the high order of accuracy for singular perturbation elliptic equations," in Investigation of Theory and Approximation Methods for Differential Equations, pp. 80-83, Turkmen State University, Ashgabat, Turkmenistan, 1991 (Russian).

[101] A. Ashyralyev and H. O. Fattorini, "On uniform difference schemes for second order singular perturbation problems in Banach spaces," SIAM Journal on Mathematical Analysis, vol. 23, no. 1, pp. 29-54, 1992.

[102] M. Ashyraliyev, "A note on the stability of the integraldifferential equation of the hyperbolic type in a Hilbert space," Numerical Functional Analysis and Optimization, vol. 29, no. 78, pp. 750-769, 2008.

[103] M. Ashyraliyev and Z. Direk, "Finite difference method for the integral-differential equation of the hyperbolic type," AIP Conference Proceedings, vol. 1470, no. 1, pp. 266-269, 2012.

[104] Z. Direk and M. Ashyraliyev, "FDM for the integral-differential equation of the hyperbolic type," Advances in Difference Equations, vol. 2014, no. 1, article 132, 2014.

[105] M. Ashyraliyev and Z. Direk, "A second order of accuracy finite difference scheme for the integral-differential equation of the hyperbolic type," AIP Conference Proceedings, vol. 1611, no. 1, pp. 398-403, 2014.

[106] A. Ashyralyev, E. Misirli, and O. Mogol, "A note on the integral inequalities with two dependent limits," Journal of Inequalities and Applications, vol. 2010, Article ID 430512, 2010. 
[107] P. E. Sobolevskii, "On the equations of the second order with a small parameter at the highest derivatives," Uspekhi Matematicheskikh Nauk, vol. 19, no. 6, pp. 217-219, 1964 (Russian).

[108] P. E. Sobolevskii and S. Semenov, "On some approach to investigation of singular hyperbolic equations," Doklady Akademii Nauk SSSR, vol. 270, no. 1, pp. 555-558, 1983 (Russian).

[109] M. Ashyraliyev, "Integral inequalities with four variable limits," in Modeling Processes of Exploitation of Gas Places and Applied Problems of Theoretical Gasohydrodynamics, pp. 170-184, Ilim, Ashgabat, Turkmenistan, 1998, (Russian).

[110] M. Ashyraliyev, "On stability estimates of Goursat differential and difference problems for hyperbolic equations," in Some Problems of Applied Mathematics, pp. 103-112, Fatih University, Istanbul, Turkey, 2000.

[111] M. Ashyraliyev and A. Ataev, On Stability Estimates of Goursat Problem for Hyperbolic Equations, 8 Years Turkmenistan's Independence, Ilim, Ashgabat, Turkmenistan, 1999.

[112] A. Ashyralyev and R. Prenov, "The hyperbolic system of equations with nonlocal boundary conditions," TWMS Journal of Applied and Engineering Mathematics, vol. 2, no. 2, pp. 154178, 2012.

[113] A. Ashyralyev and R. Prenov, "Finite-difference method for the hyperbolic system of equations with nonlocal boundary conditions," Advances in Difference Equations, vol. 2014, no. 1, article 26, 2014.

[114] H. Soltanov, "A note on the Goursat problem for a multidimensional hyperbolic equation," Contemporary Analysis and Applied Mathematics, vol. 1, no. 2, pp. 98-106, 2013.

[115] J. A. Goldstein, Semigroups of Linear Operators and Applications, Oxford Mathematical Monographs, The Clarendon Press Oxford University Press, New York, NY, USA, 1985.

[116] P. E. Sobolevskii, Difference Methods for the Approximate Solution of Differential Equations, Izdat, Voronezh Gosud University, Voronezh, Russia, 1975 (Russian).

[117] A. Ashyralyev and O. Yildirim, "On the numerical solution of hyperbolic IBVP with high-order stable finite difference schemes," Boundary Value Problems, vol. 2013, article 29, 34 pages, 2013.

[118] A. Ashyralyev and O. Yildirim, "High order accurate difference schemes for hyperbolic IBVP," in Numerical Analysis and Its Applications, vol. 8236 of Lecture Notes in Computer Science, pp. 174-181, Springer, 2013.

[119] D. Amanov and A. Ashyralyev, "Well-posedness of boundary value problems for partial differential equations of even order," Electronic Journal of Differential Equations, vol. 2014, no. 108, pp. 1-18, 2014.

[120] A. Ashyralyev and N. Aggez, "A note on the difference schemes of the nonlocal boundary value problems for hyperbolic equations," Numerical Functional Analysis and Optimization, vol. 25, no. 5-6, pp. 439-462, 2004.

[121] D. Bazarov and H. Soltanov, Some Local and Nonlocal Boundary Value Problems for Equations of Mixed and Mixed-Composite Types, Ilim, Ashgabat, Turkmenistan, 1995, (Russian).

[122] T. D. Djuraev, Boundary Value Problems for Equations of Mixed and Mixed Composite Types, FAN, Tashkent, Uzbekistan, 1978, (Russian).

[123] T. Sh. Kalmenov, Boundary Value Problems for Linear Partial Differential Equations of Hyperbolic Type, Gylym, Shymkent, Kazakhstan, 1993, (Russian).

[124] M. S. Salakhitdinov, Equations of Mixed-Composite Type, FAN, Tashkent, Uzbekistan, 1974, (Russian).
[125] V. N. Vragov, Boundary Value Problems for Nonclassical Equations of Mathematical Physics, Text-Book for Universities, NGU, Novosibirsk, Russia, 1983, (Russian).

[126] A. Ashyralyev and Y. Ozdemir, "On numerical solutions for hyperbolic-parabolic equations with the multipoint nonlocal boundary condition," Journal of the Franklin Institute, vol. 351, no. 2, pp. 602-630, 2014.

[127] D. Amanov and A. Ashyralyev, "Initial-boundary value problem for fractional partial differential equations of higher order," Abstract and Applied Analysis, vol. 2012, Article ID 973102, 16 pages, 2012. 


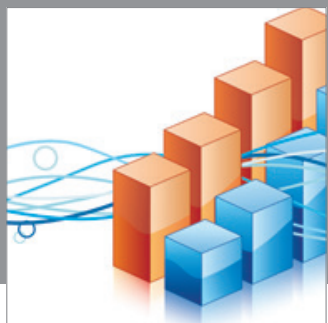

Advances in

Operations Research

mansans

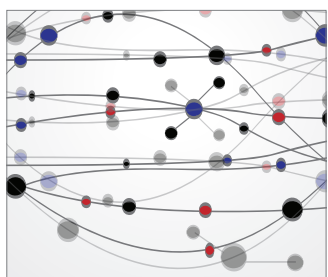

The Scientific World Journal
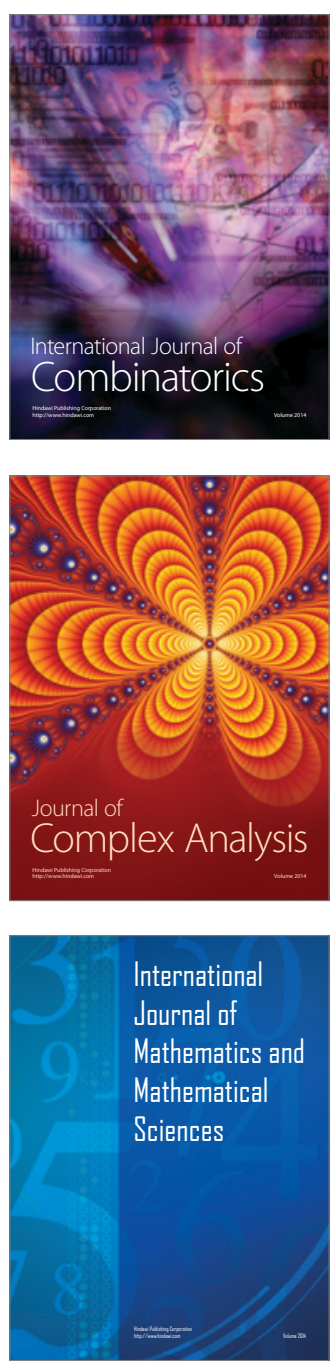
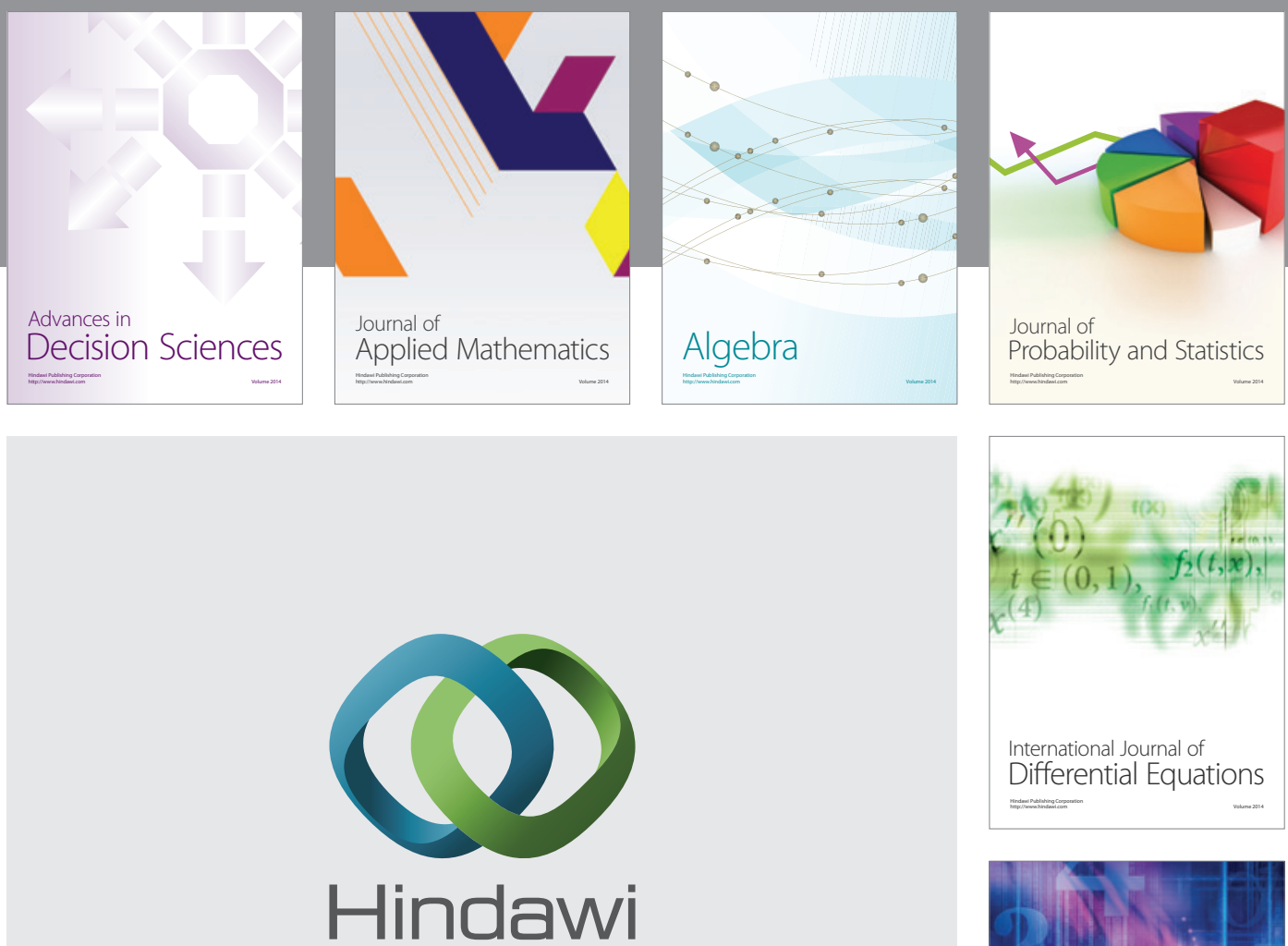

Submit your manuscripts at http://www.hindawi.com
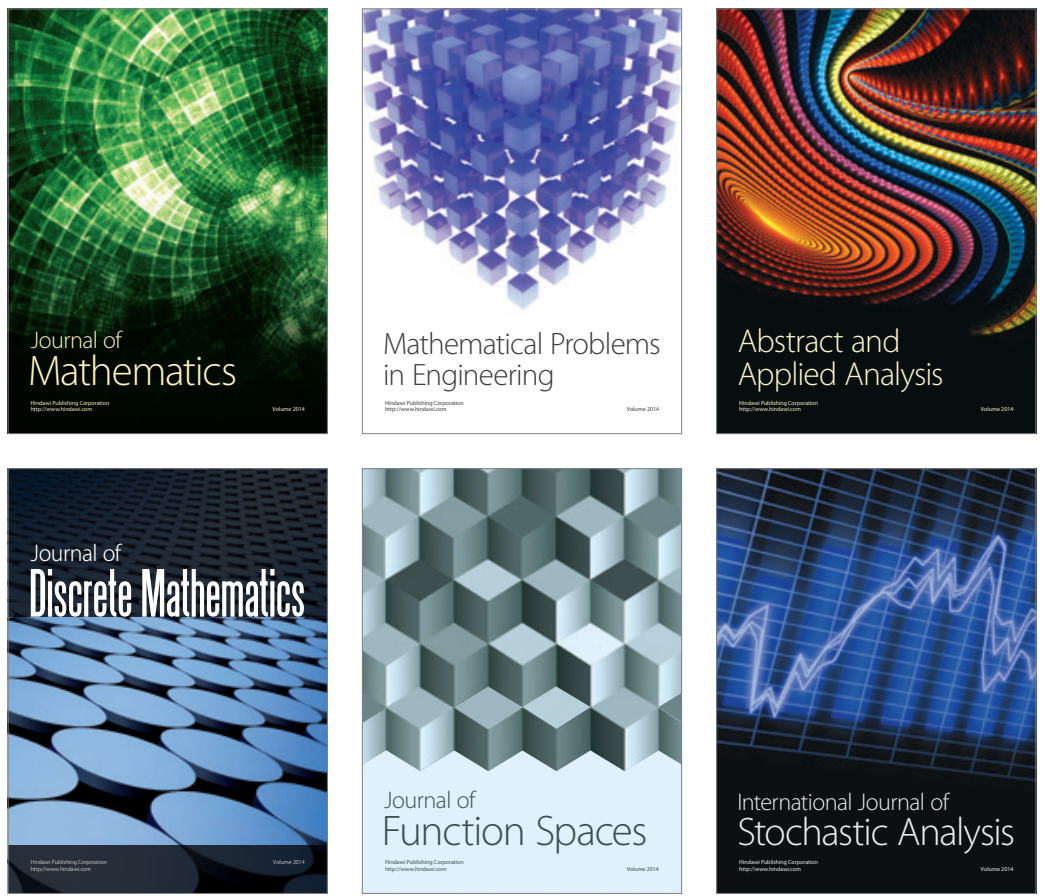

Journal of

Function Spaces

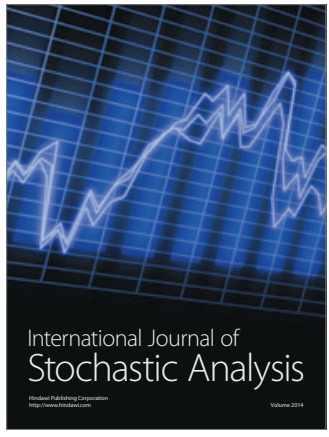

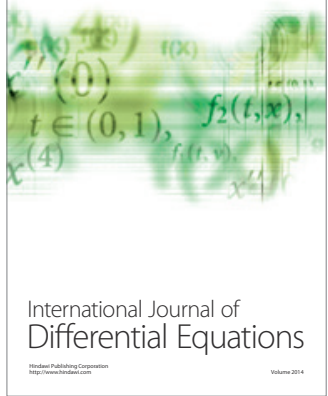
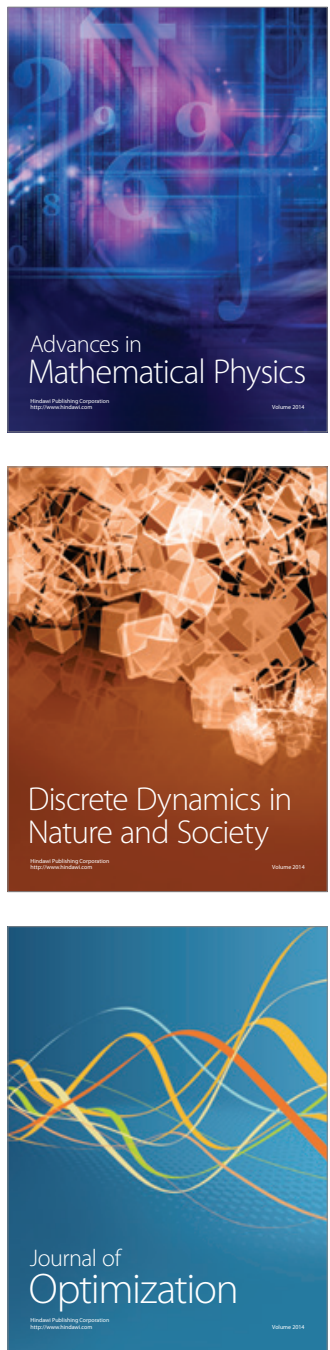